\title{
Kombinierte Längs- und Quertrajektorienplanung für automatisierte Fahrstreifenwechsel
}

\section{Dipl.-Ing. Tim Hansen}







\title{
Kombinierte Längs- und Quertrajektorienplanung für automatisierte Fahrstreifenwechsel
}

\author{
Vom Fachbereich \\ Elektrotechnik und Informationstechnik \\ der Technischen Universität Darmstadt \\ zur Erlangung der Würde eines Doktor-Ingenieurs (Dr.-Ing.) \\ genehmigte Dissertation \\ von
}

Dipl.-Ing. Tim Hansen

geboren in Rendsburg

Referent: Prof. Dr.-Ing. Ulrich Konigorski

Korreferent: $\quad$ Prof. Dr.-Ing. Ansgar Trächtler

Tag der Einreichung: 26. März 2018

Tag der mündlichen Prüfung: 19. Juli 2018



D 17

Darmstädter Dissertationen 
Kombinierte Längs- und Quertrajektorienplanung für automatisierte Fahrstreifenwechsel Gemehmigte Dissertation von Dipl.-Ing. Tim Hansen

D $17 \cdot$ Darmstadt 2018

\section{Elektronische Version}

Bereitgestellt von tuprints, E-Publishing-Service der TU Darmstadt

http://tuprints.ulb.tu-darmstadt.de

tuprints@ulb.tu-darmstadt.de

Bitte zitieren Sie dieses Dokument als:

URN: urn:nbn:de:tuda-tuprints-80812

URL: http://tuprints.ulb.tu-darmstadt.de/8081

\section{Gebundene Version}

Erhältlich im Buchhandel oder bei:

epubli GmbH, Berlin, www.epubli.de

ISBN 978-3-746778-33-4

\section{Lizenz}

\section{(c) (1) (9) $(\Theta$}

Die Veröffentlichung steht unter folgender Creative-Commons-Lizenz:

Namensnennung - Nicht-kommerziell - Keine Bearbeitung 4.0 International

https://creativecommons.org/licenses/by-nc-nd/4.0/deed.de

Weitere darüberhinausgehende Nutzungsrechte auf Anfrage möglich:

publications@timhansen.de

\section{Impressum}

Tim Hansen, c/o AutorenServices.de, Birkenallee 24, 36037 Fulda 


\section{Vorwort}

Die vorliegende Arbeit entstand während meiner Tätigkeit als wissenschaftlicher Mitarbeiter am Fachgebiet Regelungstechnik und Mechatronik der TU Darmstadt im Rahmen einer Kooperation mit der Robert Bosch GmbH.

Ganz besonderer Dank gilt hierbei natürlich meinem Doktorvater Herrn Prof. Dr.-Ing. Ulrich Konigorski, der mich hervorragend bei meiner Arbeit unterstützte. Auch besonders durch seine Art das Fachgebiet zu führen entstand eine ausgezeichnete Arbeitsatmosphäre, die den passenden Rahmen für das Gelingen dieser Arbeit bildete. Außerdem danke ich Herrn Prof. Dr.-Ing. Ansgar Trächtler für die Übernahme des Korreferats.

Natürlich gilt mein Dank auch allen anderen die mich bei dieser Arbeit unterstützt haben. Bei meinen Kollegen möchte ich mich für die sehr angenehme Zusammenarbeit bedanken, das Fahren der Umfeldfahrzeuge auf dem Testgelände und den vermutlich besten Doktorhut den man sich vorstellen kann. Bei Dr. Eric Lenz und Ingmar Gundlach bedanke ich mich für die vielen fachlichen und inspirierenden Diskussionen.

Seitens Bosch möchte ich besonders Dr. Michael Knoop, Michael Schulz und Dr. Marc Arnon erwähnen. Bei Michael Schulz möchte ich mich insbesondere für die Unterstützung bei der praktischen Umsetzung meiner Arbeit im Fahrzeug sowie für die vielen Versuchsfahrten bedanken.

Bei den Mitarbeitern des Sekretariats Brigitte Hoppe und Corina Fischer möchte ich mich ebenfalls sehr für ihre Mühe und Zeit bedanken, ebenso bei Alfred Groß aus der ElektroWerkstatt für seine große Hilfsbereitschaft sowie bei Alexander Stark und seinen Kollegen aus der Metall-Werkstatt.

Zu guter Letzt gilt mein ganz besonderer Dank natürlich auch meiner Familie, die mich bei der Arbeit unterstützte, motivierte und mir stets den Rücken frei hielt. 


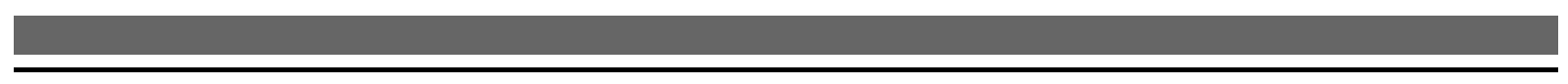




\section{Kurzfassung}

Beschrieben wird ein System, welches automatisiert Fahrstreifenwechsel auf der Autobahn durchführt. Das System setzt hierbei als Sensorik zur Umfelderfassung nur mehrere Radarsensoren und eine Monokamera voraus. Aufgrund der Zeitabhängigkeit der Umfeldobjekte ist es notwendig, anstatt einer Bahn $y(x)$ eine Trajektorie $(x(t), y(t))$ zu planen. Zur Führung des Fahrzeugs besteht das System aus einer Trajektorienplanung und einer unterlagerten Regelung. Durch diese Trennung ist es möglich, die Neuplanungsrate geringer zu wählen, als dies bei einer klassischen Modellprädiktiven Regelung der Fall wäre.

Zur Trajektorienplanung wurden zwei verschiedene Methoden entwickelt. Bei beiden Planungsmethoden ist die Trajektorie Ergebnis eines nichtlinearen Optimierungsproblems. Beide Planungen berücksichtigen durch ihre Gütefunktion die beschränkte Fahrzeugdynamik sowie weitere Komfortgrenzen. Zusätzlich berücksichtigen sie die Kollisionsfreiheit der Trajektorie und den Einfluss auf den rückwärtigen Verkehr.

Bei der „Drei-Segment-Planung“ wird der Fahrstreifenwechsel in drei Segmente unterteilt. Das erste Segment beschreibt die Anfahrt zur Wechsellücke, das zweite den eigentlichen Fahrstreifenwechsel und im dritten findet die Weiterfahrt auf dem Zielfahrstreifen statt. Je nach Situation können einzelne Segmente entfallen. Im ersten und zweiten Segment wird die Trajektorie in Längs- und Querrichtung durch je ein Polynom fünften Grades beschrieben. Im dritten Segment wird die Längsführung durch den Abstandsregeltempomaten (Adaptive Cruise Control ACC) übernommen und die Quertrajektorie, in Form einer Matrix-Exponentialfunktion, sorgt für den Ausgleich der verbliebenen Querablage. Als Optimierungsvariablen dienen hierbei drei Parameter der Längstrajektorie. Die Quertrajektorie ergibt sich indirekt aus der Längstrajektorie. Die optimalen Werte der Optimierungsvariablen werden ohne Gradienteninformation mit Hilfe des Downhill-Simplex-Algorithmus bestimmt.

Beim zweiten Verfahren „Spline-Planung“ besteht die Trajektorie aus vielen Polynomsegmenten dritten Grades. Dadurch ist es möglich, einen stetigen Verlauf der Beschleunigung zu erreichen. Die Nebenbedingungen wurden offline so eliminiert, dass sich die Abtastung auf eine MatrixMultiplikation reduzieren lässt. Die Trajektorie ist in ihrer Form weitaus flexibler als beim ersten Verfahren, da pro Segment und Richtung eine Optimierungsvariable vorhanden ist. Die Form der Trajektorie wird im Wesentlichen durch die Gütefunktion festgelegt. Diese Gütefunktion ist stetig differenzierbar, so dass sich ein Gradient berechnen lässt. Dieser wird benutzt, um das Optimum mit Hilfe des L-BFGS-Algorithmus zu bestimmen.

Beide Trajektorienplanungen wurden in einem Versuchsfahrzeug implementiert und intensiv getestet. Neben den Testfahrten auf dem August-Euler-Flugplatz in Griesheim wurde das System auch im realen Straßenverkehr erprobt. Beide Trajektorienplanungen ermöglichen komfortable 
und sichere Fahrstreifenwechsel auch in dichtem Verkehr auf der Autobahn. Sie sind zudem bei Bedarf auch in der Lage, den Fahrstreifenwechsel abzubrechen. Die Trajektorien beider Verfahren wurden von den Fahrern als komfortabel und angenehm beschrieben.

Eine Abschätzung der Rechenzeit hat ergeben, dass beide Planungsmethoden in Echtzeit auf einem herkömmlichen Steuergerät ausführbar sind. Die Spline-Planung ist zwar etwas rechenzeitintensiver, jedoch auch deutlich flexibler und einfacher zu erweitern. Zudem ist es bei der Drei-Segment-Planung notwendig, Fallunterscheidungen durchzuführen, um die Anzahl der Segmente zu bestimmen. Diese Fallunterscheidungen sind ggf. nicht für jede Situation passend. Daher wird die Spline-Planung als langfristig tragfähiger betrachtet. 


\section{Abstract}

This work describes a system that performs automated lane changes on freeways. The system requires only multiple radar sensors and one mono camera for environment detection. Because of the time dependency of the environment objects it is necessary to plan a trajectory $(x(t), y(t))$ instead of a path $y(x)$. For vehicle guidance the system consists of a trajectory planner and an inner control loop. This separation makes it possible to choose a lower replanning frequency than the one necessary for a classical model predictive control approach. Two different methods for trajectory planning have been developed in this work. In both methods the trajectory is the result of a nonlinear optimization problem. Both methods take the limited vehicle dynamics and further comfort objectives inside the cost function into account. In addition, they consider the collision avoidance and the influence on the rearward traffic.

For the „Drei-Segment-Planung“ the lane change is divided into three segments. The first segment describes the approach to the changing gap, the second the actual lane change and in the third segment the vehicle continues driving on the destination lane. Depending on the situation, some of the segments can be omitted. In the first and second segment, the trajectory is described in lateral and longitudinal direction by fifth degree polynomials. In the third segment, the vehicle is guided in longitudinal direction by the adaptive cruise control (ACC) and the remaining lateral deviation is reduced by a lateral trajectory in the form of a matrix exponential function. Three parameters of the longitudinal trajectory are used as optimization variables. The lateral trajectory is derived indirectly from the longitudinal one. The optimal values for the optimization variables are determined without gradient information with the help of the downhill simplex algorithm.

For the second method „Spline-Planung“ the trajectory consists of many different polynomial segments of degree three. By that it is possible to achieve a continuous acceleration profile. The constraints have been eliminated offline in a way that it is possible to reduce the sampling of the trajectory to a matrix multiplication. Because of one optimization variable per segment and per direction, the shape of the trajectory is much more flexible than in the first method. The shape of the trajectory is mainly defined by the cost-function. This cost-function is continuously differentiable and therefore it is possible to calculate a gradient. This gradient is used to find the optimum by means of the L-BFGS algorithm.

Both methods have been implemented in an experimental vehicle and have been tested intensively. Beside the test drives at the August-Euler airfield near Darmstadt the system has been tested in real world traffic. Both methods allow comfortable and save lane changes also in dense traffic on freeways. If necessary they are both capable of aborting the lane change. The trajectories of both methods have been described as comfortable and pleasant by the drivers. 
An estimation of the calculation time has shown that it is possible to execute both methods on a regular electronic control unit. The spline planner needs some more calculation time, but is more flexible and easier to extend. In addition it is necessary to perform case distinctions for the three segment planner to determine the number of segments. These distinctions might be inappropriate for certain situations. Therefore, the spline planner is considered to be more sustainable in the long run. 


\section{Inhaltsverzeichnis}

Vorwort

Kurzfassung iii

Abstract $\quad$ v

Symbole und Abkürzungen $\quad$ xi

1 Einleitung 1

1.1 Stand der Technik . . . . . . . . . . . . . . . . . . . . 1



1.3 Gliederung .......................... 3

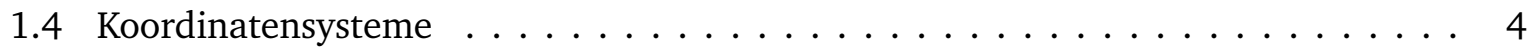

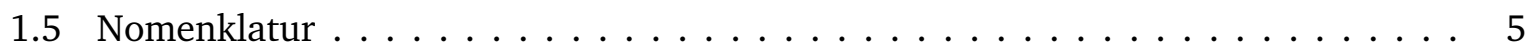

2 Sensorik \& Vorverarbeitungen $\quad 7$



2.2 Lokalisierung im Straßenkoordinatensystem $\ldots \ldots \ldots \ldots \ldots \ldots$

2.3 Verarbeitung der Umfeldfahrzeuge $\ldots \ldots \ldots \ldots \ldots \ldots$

2.4 Bestimmung geeigneter Lücken $\ldots \ldots \ldots \ldots \ldots \ldots$

2.5 Wunschfahrstreifen des Fahrers . . . . . . . . . . . . . . . . . . . . 10

2.6 Auswahl der Ziellücke . . . . . . . . . . . . . . . . . . . . . 11

2.7 Struktur der Regelung und Trajektorienplanung . . . . . . . . . . . . . 12

3 Einführung in die Trajektorienplanung $\quad 15$

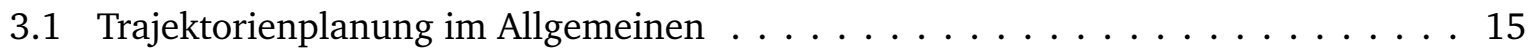

3.2 Trajektorienplanung für Fahrstreifenwechsel $\ldots \ldots \ldots \ldots \ldots \ldots$

3.3 Verfahren aus der Literatur . . . . . . . . . . . . . . . . . . . . . . . . 19

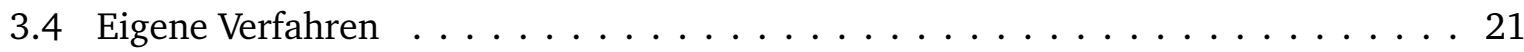

3.5 Weitere Ansätze . . . . . . . . . . . . . . . . . . . . . . . 24

4 Drei-Segment-Planung 25

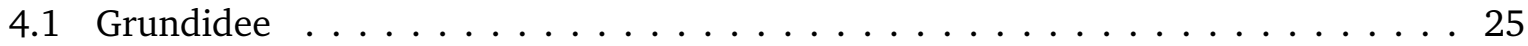



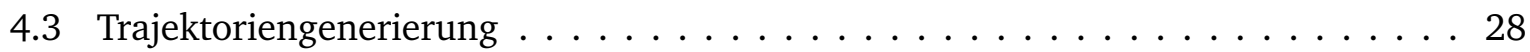

4.3.1 Generierung der Längstrajektorie . . . . . . . . . . . . . . . . . . . . . 29 
4.3.2 Generierung der Quertrajektorie . . . . . . . . . . . . . . . . . 31

4.4 Abtastung der Trajektorie . . . . . . . . . . . . . . . . . . . 33

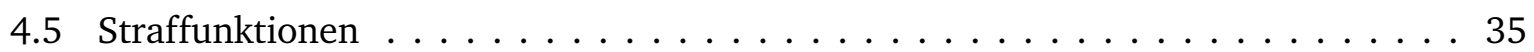

4.5.1 $J_{\text {SafetyArea }}$ - Bewertung der Annäherung an die Sicherheitsbereiche . . . . 37

4.5.2 $J_{\text {TrafInfl }}-$ Bewertung des Einflusses auf den rückwärtigen Verkehr . . . . . 40

4.5.3 $J_{\text {EgoDynLim }}-$ Beschränkung der Trajektoriendynamik . . . . . . . . . . . . 42

4.5.4 Bewertung der Endsituation in Längsrichtung . . . . . . . . . . . . . . . 44

4.6 Bestimmung der optimalen Trajektorie . . . . . . . . . . . . . . . . . 45

4.7 Ergebnisse . . . . . . . . . . . . . . . . . . . . . . . 47



5 Spline-Planung $\quad 51$

5.1 Grundidee . . . . . . . . . . . . . . . . . . . . . . . 51

5.2 Generierung der Trajektorie . . . . . . . . . . . . . . . . . . . . 51



5.4 Überblick . . . . . . . . . . . . . . . . . . . . . . . . 56



5.5.1 Prädiktion mit konstanter Geschwindigkeit . . . . . . . . . . . . . . 57

5.5.2 Prädiktion unter Ausnutzung der Beschleunigung . . . . . . . . . . . . . 58

5.5.3 Prädiktion der Umfeldobjekte mit Beschleunigung und Fahrermodell . . . . 59

5.6 Straffunktionen . . . . . . . . . . . . . . . . . . . . . . . . . . . 59

$5.6 .1 J_{\mathrm{AC}}-$ Kollisionsvermeidung $\ldots \ldots \ldots \ldots \ldots \ldots$

5.6.2 Einfluss auf den rückwärtigen Verkehr . . . . . . . . . . . . . . . . . 62

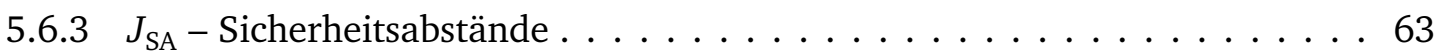

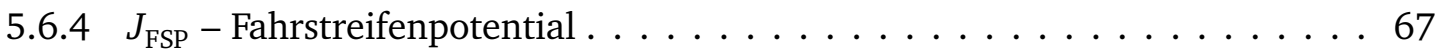

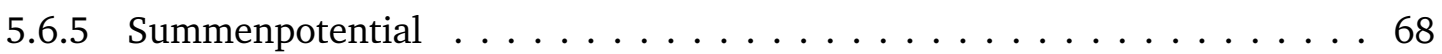

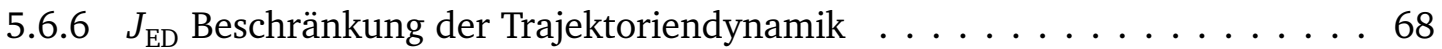

5.6.7 Bewertung des Endpunktes in Längsrichtung . . . . . . . . . . . . . . . . . 69

5.7 Summation der Straffunktionen $\ldots \ldots \ldots \ldots \ldots \ldots$. . . . . . . . . . 71

5.8 Bestimmung der optimalen Trajektorie . . . . . . . . . . . . . . . . . . 72

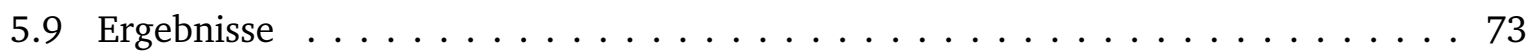

5.10 Rechenzeit . . . . . . . . . . . . . . . . . . . . . . . . 74

6 Ergebnisse \& Vergleich $\quad 75$

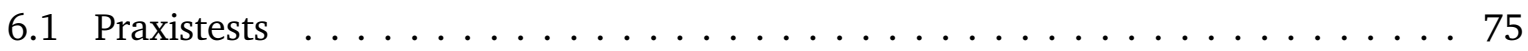

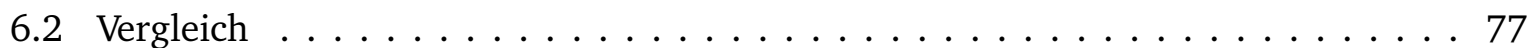

6.2.1 Vergleich der Trajektorien $\ldots \ldots \ldots \ldots \ldots$. . . . . . . . 78

6.2.2 Vergleich der Konvergenz der beiden Planungsverfahren . . . . . . . . . 80

6.2 .3 Vergleich der Berechnungszeiten . . . . . . . . . . . . . . . . . . . 84

6.2.4 Stärken und Schwächen der beiden Planungsmethoden . . . . . . . . . . . 84

6.3 Erweiterungsmöglichkeiten . . . . . . . . . . . . . . . . . . . 85 
6.4 Empfehlung . . . . . . . . . . . . . . . . . . . . . . . . . . 89

7 Zusammenfassung \& Ausblick $\quad 91$

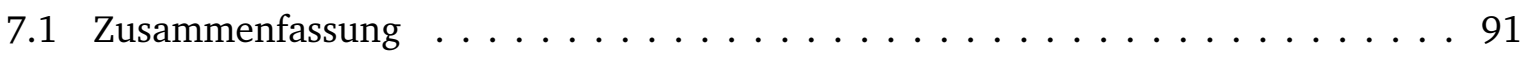

7.2 Ausblick . . . . . . . . . . . . . . . . . . . . . . 92

A Detailbetrachtungen der Drei-Segment-Planung 95

A.1 Generierung der Drei-Segment-Trajektorie . . . . . . . . . . . . . . . 95

A.1.1 Längsrichtung . . . . . . . . . . . . . . . . . . . . . . . . . . . . 98

A.1.2 Querrichtung ... . . . . . . . . . . . . . . . . . 102

A.2 Details zur Generierung des 3. Segmentes . . . . . . . . . . . . . . . . . 105

A.3 Vergleich unterschiedlicher Parametrierungen der Quertrajektorie . . . . . . . . . 108

A.4 Bestimmung der optimalen Fahrstreifenwechseldauer ohne Umfeldfahrzeuge . . . 110

A.5 Begrenzung von $\bar{v}_{1}$ und $\bar{v}_{2}$ zur Einschränkung der Startwerte . . . . . . . . . 111

A.6 Die unterschiedlichen Startzustände der Planung (Fallunterscheidungen) . . . . . . 114

A.7 Abtastzeitenbetrachtung . . . . . . . . . . . . . . . . . . . . . . . 116

B Detailbetrachtungen der Spline-Planung 117

B.1 Generierung der Spline-Trajektorie . . . . . . . . . . . . . . . . . . . . . 117

B.1.1 Integraler Güteterm . . . . . . . . . . . . . . . . . . . . . . . . 120

B.1.2 Endpunktbewertung . . . . . . . . . . . . . . . . . . . . . . . 121

B.1.3 Abtastung . . . . . . . . . . . . . . . . . . . . . . . . 122

B.2 Alternative Parametrierungsmöglichkeiten . . . . . . . . . . . . . . . . . . . 124

B.3 Bestimmung der überschüssigen Energie am Endpunkt . . . . . . . . . . . . . . . 126

B.4 Fahrstreifenpotential . . . . . . . . . . . . . . . . . . . . . . 132

C Vergleich der Trajektorienplanungen in unterschiedlichen Szenarien 139

D Kritische Szenarien für die Trajektorienplanung 151

E Optimierung mit Nebenbedingungen 155

F Der Versuchsträger 157



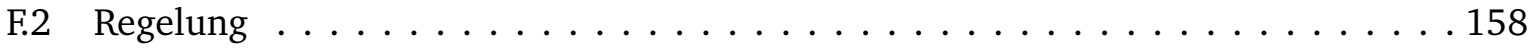

F.2.1 Identifikation der Längsaktorik . . . . . . . . . . . . . . . . . . 158

F.2.2 Längsregler . . . . . . . . . . . . . . . . . . . . . . . . . . . . . . . 160

F.3 Filterung der Fahrstreifenbelegungswahrscheinlichkeiten . . . . . . . . . . . . . . 161

$\begin{array}{ll}\text { Literaturverzeichnis } & 163\end{array}$ 


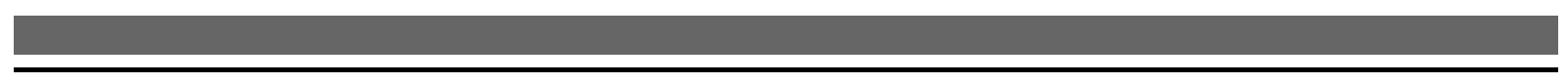




\section{Symbole und Abkürzungen}

\begin{tabular}{lll}
\multicolumn{2}{l}{ Allgemeine Symbole und Formelzeichen } \\
Symbol & Beschreibung & Einheit \\
\hline$p$ & Position & $\mathrm{m}$ \\
$v$ & Geschwindigkeit & $\mathrm{m} / \mathrm{s}$ \\
$a$ & Beschleunigung & $\mathrm{m} / \mathrm{s}^{2}$ \\
$j$ & Ruck & $\mathrm{m} / \mathrm{s}^{3}$ \\
$t$ & Zeit & $\mathrm{s}$ \\
$s$ & Strecke & $\mathrm{m}$ \\
$J$ & Gütemaß, Güteterm & \\
$\phi$ & Potential für Güteterm & \\
$w$ & Gewichtungsfaktoren & \\
$\underline{\mathbf{W}}$ & Gewichtungsmatrix & \\
$k$ & Parameter der Straffunktion & \\
$\underline{\mathbf{x}}$ & Zustandsvektor & \\
$v_{\text {Beobachter }}$ & Geschwindigkeit des Beobachters, auf den $p_{\mathrm{x}, \text { rel }}$ bezogen ist & $\mathrm{m} / \mathrm{s}$ \\
$T_{\text {Plan }}$ & Neuplanungszeit & $\mathrm{s}$
\end{tabular}

\section{Symbole und Formelzeichen bei der Drei-Segment-Planung}

\begin{tabular}{lll} 
Symbol & Beschreibung & Einheit \\
\hline $\bar{v}_{1}$ & Durchschnittsgeschwindigkeit im ersten Segment & $\mathrm{m} / \mathrm{s}$ \\
$\bar{v}_{2}$ & Durchschnittsgeschwindigkeit im zweiten Segment & $\mathrm{m} / \mathrm{s}$ \\
$v_{\mathrm{e}}$ & Geschwindigkeit am Ende des zweiten Segmentes & $\mathrm{m} / \mathrm{s}$
\end{tabular}

a, b Vektoren der Polynomkoeffizienten 


\section{Symbole und Formelzeichen bei der Spline-Planung}

Symbol Beschreibung Einheit

$T \quad$ Dauer eines Segmentes S S

$\Delta T \quad$ zeitlicher Abstand zwischen zwei Abtastpunkten $\quad \mathrm{S}$

$n \quad$ Anzahl der Segmente

$m \quad$ Anzahl der Abtastpunkte pro Segment

p Polynomkoeffizienten

s $\quad$ Abtastpunkte

j Vektor mit den Optimierungsvariablen (hier Rucke)

Indizes

Symbol Beschreibung

$(\ldots)^{\mathrm{r}} \quad$ im Straßenkoordinatensystem

$(\ldots)^{\mathrm{b}} \quad$ im Fahrzeugkoordinatensystem

(...) Lon Längsrichtung

(...) Lat Querrichtung

$(\ldots)_{\text {rel }} \quad$ relativ zu einem bewegten Beobachter mit konstanter Geschwindigkeit

$(\ldots)_{i} \quad$ üblicherweise: das $i$. Segment bzw. der $i$. Abtastpunkt

Weitere Indizes und deren Beschreibung befinden sich in Abschnitt 1.5 Nomenklatur. 


\section{Abkürzungen}

Kürzel vollständige Bezeichnung

FD vorausfahrendes Fahrzeug auf dem Zielfahrstreifen (Front Destination Lane)

FS vorausfahrendes Fahrzeug auf dem Startfahrstreifen (Front Start Lane)

TD rückwärtiges Fahrzeug auf dem Zielfahrstreifen (Tail Destination Lane)

TS rückwärtiges Fahrzeug auf dem Startfahrstreifen (Tail Start Lane)

S... $\quad$ Grenze des Sicherheitsbereiches des Fahrzeugs ...

SA Sicherheitsbereich (Safety Area)

AC Kollisionsvermeidung (Anti-Collision)

FSP Fahrstreifenpotential

IDM Intelligent Driver Model [Treiber, 2010]

Ego eigenes Fahrzeug

ACC Abstandsregeltempomat (Adaptive Cruise Control)

LRR Fernbereichsradarsensor (Long-Range-Radar)

MRR Mittelbereichsradarsensor (Mid-Range-Radar)

EPS elektrische Servolenkung (Electric Power Steering)

L-BFGS Limited-memory Broyden-Fletcher-Goldfarb-Shanno-Algorithmus

CANape Mess-Software der Firma Vector Informatik

MPC Modellprädiktive Regelung (Model Predictive Control)

ID Identifikationsnummer

SAE amerikanische Standardisierungsorganisation (Society of Automotive Engineers) 







\section{Einleitung}

Im Laufe der letzten Jahre haben Fahrerassistenzsysteme eine immer größere Bedeutung erfahren. Der Abstandsregeltempomat (ACC) ist so heutzutage auch schon in der Kleinund Kompaktwagenklasse verfügbar. Auch die Unterstützung der Fahrzeugquerführung im Rahmen einer Fahrstreifenmittenführung verbreitet sich zunehmend. Bei einem manuellen Fahrstreifenwechsel wird die Längsführung des ACCs etwas angepasst, ohne jedoch garantieren zu können, dass diese Bewegung auch für den Fahrstreifenwechsel geeignet ist [Winner, 2015, S. 877]. Ein logischer nächster Schritt ist es daher, die Systeme so zu erweitern, dass sie auch Fahrstreifenwechsel selbstständig durchführen können.

\subsection{Stand der Technik}

Bei der Betrachtung von Systemen zum automatisierten Fahrstreifenwechsel sind große Unterschiede in der Menge der abgedeckten Szenarien erkennbar. Bei einfachen Systemen wird beispielsweise nur eine Quertrajektorie geplant. Es wird geprüft, ob ein ausreichend großer Bereich auf dem Zielfahrstreifen frei ist. Sofern dies der Fall ist, kann der Fahrstreifenwechsel durchgeführt werden. Derartige Systeme benötigen wenig Rechenzeit, können den Fahrstreifen jedoch nur wechseln, sofern der Zielfahrstreifen weitgehend frei ist.

Bereits 1994 wurden so mit dem Versuchsfahrzeug VaMP erste automatisierte Fahrstreifenwechsel auf einer Autobahn vorgeführt [Dickmanns, 1994]. Der Fahrstreifenwechsel bezog sich hierbei ausschließlich auf die Querbewegung. Ausgehend von einer normalen Autobahnfahrt wurde ein Lenkwinkelverlauf bestimmt, der das Fahrzeug auf den Nachbarfahrstreifen führte [Dickmanns LC]. Dieser Verlauf wurde als Vorsteuerung auf das System geschaltet und war im Wesentlichen abhängig von der Fahrstreifenbreite sowie der gefahrenen Geschwindigkeit [Dickmanns, 2007, S. 85 ff.]. Auch die aktuell in der Serie auftauchenden aktiven Spurwechselassistenten konzentrieren sich auf eine Querbewegung, die durchgeführt wird, sofern der Zielfahrstreifen frei ist [Hafner, 2016, S. 74-75].

In neueren Forschungsarbeiten wurden auch Systeme gezeigt, bei denen sowohl eine Quer- als auch eine Längstrajektorie für den Fahrstreifenwechsel geplant werden. Dadurch ist es möglich, auch Geschwindigkeitsunterschiede zwischen den Fahrstreifen zu berücksichtigen, sowie aktiv eine Lücke anzufahren. Die Planung kann kombiniert oder nacheinander geschehen. Durch eine zyklische Neuplanung kann die Trajektorie auch während des Fahrstreifenwechsels noch an das sich verändernde Umfeld angepasst werden.

Bei einigen Arbeiten wird der Fahrstreifenwechsel so primär als Längsplanungsproblem aufgefasst. Es wird zunächst die Längs- und anschließend die Quertrajektorie geplant 
[Nilsson, 2015][Nilsson, 2016][Nilsson, 2017]. Dies ist günstig für die Rechenzeit, hat jedoch den Nachteil, dass die Quertrajektorie nicht auf die Längstrajektorie „einwirkt“.

Systeme mit kombinierter Längs- und Querplanung werden häufig im Bereich des hochautomatisierten Fahrens verwendet. In [Werling, 2011, S. $23 \mathrm{ff}$.] wird ein Verfahren gezeigt, bei dem die Trajektorie aus einem Polynom fünften Grades in Längs- und Querrichtung besteht. Durch Variation des Endpunktes wird eine diskrete Menge an möglichen Trajektorien erzeugt. Für alle Trajektorien aus dieser Menge wird die Güte bestimmt und anschließend die beste Trajektorie ausgewählt. Durch die Polynomform der Trajektorie ist es im Allgemeinen nicht möglich, die Maximalbeschleunigung über einen gewissen Zeitraum aufrecht zu halten. Neben anderem wurde dies in [Rathgeber, 2016a] verbessert. Bei beiden Ansätzen werden jedoch nur Trajektorien betrachtet, bei denen die Querbewegung sofort beginnt. Im Rahmen der BerthaBenz-Fahrt 2013 wurde ein Verfahren eingesetzt, bei dem die Trajektorie aus einem Polygonzug besteht. Die Kollisionsfreiheit wurde hierbei über harte nichtlineare Nebenbedingungen erreicht [Ziegler, 2015, S.87ff.]. Zur Lösung wurde das SQP-Verfahren eingesetzt (Sequenzielles quadratisches Programmieren).

Die erwähnten Verfahren zum hochautomatisierten Fahren fokussieren sich hierbei nicht so sehr auf den Fahrstreifenwechsel, sondern planen allgemein Trajektorien für unterschiedlichste Verkehrsszenarien. Diese Arbeit dagegen betrachtet explizit die Eigenarten und Besonderheiten der Planung von Fahrstreifenwechseltrajektorien.

\subsection{Projektbeschreibung}

Die Arbeiten zu dieser Dissertation fanden im Rahmen einer Kooperation zwischen dem Fachgebiet Regelungstechnik und Mechatronik der TU Darmstadt und der Robert Bosch GmbH statt. Im Rahmen des Projektes sollte ein System zur Trajektorienplanung für automatisierte Fahrstreifenwechsel auf der Autobahn entwickelt und in einem Versuchsfahrzeug umgesetzt werden. Die Trajektorien für Längs- und Querrichtung sollen hierbei kombiniert geplant werden. Das System soll weiterhin in der Lage sein, den Fahrstreifenwechsel abzubrechen, sofern dies erforderlich ist. Die verwendeten Algorithmen sollen möglichst rechenzeiteffizient sein, um eine Umsetzung auf einem Steuergerät zu ermöglichen.

Das System ist hierbei in den Bereich des teilautomatisiertes Fahrens einzuordnen (SAE Level 2). Unter Umständen könnte es auch für hochautomatisiertes Fahren geeignet sein (SAE Level 3) [SAE J3016, S.19f.]. Es wird angenommen, dass der Fahrer das System überwacht und als Rückfallebene zur Verfügung steht. In Gefahrensituationen kann es notwendig sein, dass andere Systeme, wie z. B. ein Notbremsassistent, die Fahrzeugführung übernehmen. Dies ist auch dadurch begründet, dass die Funktion die Längsaktorik im Versuchsfahrzeug nur im Beschleunigungsbereich der ACC-Funktion ansteuern kann (Maximalverzögerung: $\approx 3,5 \mathrm{~m} / \mathrm{s}^{2}$ ).

Im Rahmen vorangegangener Arbeiten wurden bereits automatisierte Fahrstreifenwechsel auf der Autobahn durchgeführt. Die Längsführung wurde hierbei durch das ACC übernommen. In 
Querrichtung wurde geprüft, ob sich auf dem Zielfahrstreifen ein Fahrzeug befindet oder mit kritischer Geschwindigkeit nähert. Sofern dies nicht der Fall war und der Fahrer dies wünschte, wurde in Querrichtung eine Bahn gemäß einem offline optimierten Muster abgefahren. Die Bewegungen in Längs- und Querrichtung waren dabei weitestgehend unabhängig. In Querrichtung kam als Bahn ein Polynom fünften Grades zum Einsatz, für das nachgewiesen wurde, dass es einen ruckoptimalen Verlauf darstellt.

Die vorliegende Arbeit baut auf diesen Ergebnissen auf. Dazu wurde der Versuchsträger übernommen und an die neuen Anforderungen angepasst.

\subsection{Gliederung}

Im folgenden Kapitel 2 „Sensorik \& Vorverarbeitungen“ wird auf die verwendete Sensorik des Fahrzeugs eingegangen. Es wird die Erzeugung des Straßenkoordinatensystems sowie die Vorverarbeitung der Umfeldinformationen beschrieben. Es werden mögliche Lücken, in die gewechselt werden kann, bestimmt und die Struktur der Regelung dargestellt.

Kapitel 3 „Einführung in die Trajektorienplanung“ beschreibt zunächst allgemeine Trajektorienplanungsmethoden aus der Literatur. Danach wird näher auf die Besonderheiten der Planung von Fahrstreifenwechseltrajektorien eingegangen. Es werden einige ausgewählte Projekte und deren Verfahren zur Planung derartiger Trajektorien dargestellt. Anschließend wird das Problem näher analysiert und die eigenen Lösungsansätze werden vorgestellt.

In den darauffolgenden Kapiteln 4 und 5 („Drei-Segment-Planung“ und „Spline-Planung“) werden die beiden entwickelten Trajektorienplanungsmethoden beschrieben. Hierbei wurde Wert auf eine möglichst kompakte Darstellung gelegt. Die Details zu den Planungen befinden sich im Anhang.

In Kapitel 6 „Ergebnisse \& Vergleich“ werden die Ergebnisse der Praxistests der beiden Trajektorienplanungen beschrieben, die beiden Verfahren in verschiedener Hinsicht verglichen und deren Erweiterungsmöglichkeiten dargestellt. Abschließend wird eine Empfehlung abgegeben, welche Planungsmethode weiter zu verfolgen ist.

Kapitel 7 „Zusammenfassung \& Ausblick“ fasst abschließend die Arbeit zusammen und gibt einen Hinweis auf weitere Forschungsmöglichkeiten.

Im Anhang befinden sich dann, neben den Details zu den Planungsmethoden, eine Reihe von Szenarien, in denen die Trajektorien der beiden Methoden verglichen werden. Zusätzlich wird auf einige Details des Versuchsträgers eingegangen. 


\subsection{Koordinatensysteme}

Die Welt wird in dieser Arbeit zweidimensional betrachtet. Höhenunterschiede werden daher nicht berücksichtigt. Die Ausrichtung der Koordinatenachsen orientiert sich an den gängigen Normen [DIN ISO 8855]. Verwendet werden folgende Koordinatensysteme (Abbildung 1.1):

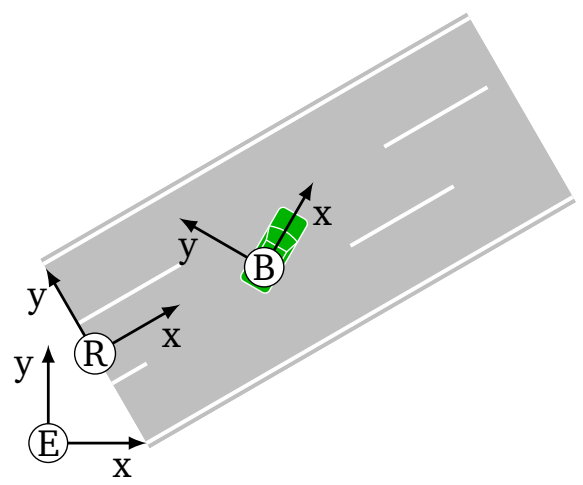

\section{Abbildung 1.1: Übersicht der verwendeten Koordinatensysteme}

Earth Das Erdkoordinatensystem ist mit seinem Ursprung fest an einem Punkt an der Erdoberfläche befestigt, die x-Achse zeigt nach Osten, die y-Achse nach Norden.

Body Das Fahrzeugkoordinatensystem liegt mit seinem Ursprung auf der Mitte der Hinterachse des eigenen Fahrzeugs und ist fest mit ihm verbunden. Die x-Achse zeigt in FahrzeugLängsachse, die y-Achse nach links.

Road Beim Straßenkoordinatensystem handelt es sich nicht um ein kartesisches Koordinatensystem. Der Ursprung des Koordinatensystems liegt beim Start in der Mitte des EgoFahrstreifens. Die x-Koordinate beschreibt die zurückgelegte Strecke in Richtung der Fahrstreifen, die y-Koordinate die Querablage (positiv nach links). Details zu diesem Koordinatensystem befinden sich in Abschnitt 2.2. 


\subsection{Nomenklatur}

Innerhalb dieser Arbeit wird für physikalische Größen eine einheitliche Notation verwendet. Diese orientiert sich an den Arbeiten im Bereich der Navigation [Wendel, 2011]. So beschreibt beispielsweise

$$
\vec{v}_{\mathrm{ec}}^{\mathrm{r}}
$$

die Geschwindigkeit $v$ in Straßenkoordinaten $r$ des Fahrzeuges c gegenüber der Erde e.

Für die physikalischen Größen werden folgende Symbole verwendet:

\begin{tabular}{c|l} 
Abk. & Bezeichnung \\
\hline$p$ & Position \\
$v$ & Geschwindigkeit \\
$a$ & Beschleunigung \\
$j$ & Ruck
\end{tabular}

Folgende Abkürzungen werden für Koordinatensysteme verwendet:

\begin{tabular}{c|l} 
Abk. & Bezeichnung \\
\hline e & (Earth) erdfestes Koordinatensystem \\
b & (Body) Fahrzeugkoordinatensystem \\
r & (Road) Straßenkoordinatensystem
\end{tabular}

Folgende Abkürzungen werden für Punkte verwendet:

\begin{tabular}{c|l} 
Abk. & Bezeichnung \\
\hline e & (Earth) Ursprung des erdfesten Koordinatensystems \\
c & (Car) ein anderes Fahrzeug \\
t & (Trajectory) die Trajektorie \\
r & (Road) Ursprung des Straßenkoordinatensystems \\
l & (Lane) Mitte des aktuellen Ego-Fahrstreifens \\
b & (Body) Mitte Hinterachse des Ego-Fahrzeugs
\end{tabular}

Ist anstatt der vektoriellen Größe nur eine Richtungskomponente gemeint, so wird der Name der Koordinatenachse angehängt. Die Position in x-Richtung eines Fahrzeugs in Straßenkoordinaten in Bezug auf dessen Ursprung lautet somit:

$$
p_{\mathrm{rc}, \mathrm{x}}^{\mathrm{r}}
$$

Aus Gründen der Übersichtlichkeit wird teilweise eine verkürzte Darstellung ohne Koordinatensystemangabe verwendet. In diesem Fall ist immer die Größe im Straßenkoordinatensystem bezüglich dessen Ursprungs gemeint. Aus dem gleichen Grund werden teilweise anstatt der Koordinatenachsen $\mathrm{x}$ und y die Bezeichnungen Lon und Lat verwendet:

$$
p_{\text {Lon }}=p_{\mathrm{rc}, \mathrm{x}}^{\mathrm{r}}
$$




\section{Vektoren und Matrizen}

Skalare, Vektoren und Matrizen werden in dieser Arbeit wie folgt dargestellt:

\begin{tabular}{c|c|l} 
Typ & Beispiel & Darstellung \\
\hline Skalar & $v$ & kursiv \\
Vektor & $\underline{\mathbf{x}}$ & klein, fett, aufrecht, unterstrichen \\
Matrix & $\underline{\mathbf{W}}$ & groß, fett, aufrecht, unterstrichen
\end{tabular}

Grundsätzlich handelt es sich bei $\underline{x}$ um einen stehenden, und folglich bei $\underline{x}^{\mathrm{T}}$ um einen liegenden Vektor. Hierbei steht $(\cdot)^{\mathrm{T}}$ für die Vektor-Transposition.

\section{Einheiten}

In der Regel werden in dieser Arbeit SI-Einheiten verwendet. Meistens werden die Einheiten mit angegeben, in einigen Fällen jedoch aus Gründen der Übersichtlichkeit weggelassen. In Fällen in denen keine SI-Einheiten verwendet werden, ist dies entsprechend gekennzeichnet.

\section{Konventionen für Ableitungen}

Für die Ableitung nach vektoriellen Größen gibt es unterschiedliche Konventionen. Da in dieser Arbeit überwiegend mit stehenden Vektoren gearbeitet wird, wird hier die Ableitung als liegender Vektor definiert:

$$
\frac{\mathrm{d} f}{\mathrm{~d} \underline{\mathbf{x}}}=\left[\begin{array}{llll}
\frac{\mathrm{d} f}{\mathrm{~d} x_{1}} & \frac{\mathrm{d} f}{\mathrm{~d} x_{2}} & \cdots & \frac{\mathrm{d} f}{\mathrm{~d} x_{n}}
\end{array}\right]
$$

Die Ableitung einer vektoriellen Größe entspricht somit der Jacobi-Matrix und es gelten folgende Regeln:

$$
\frac{\mathrm{d}}{\mathrm{d} \underline{\mathbf{x}}}(\underline{\mathbf{C}} \cdot \underline{\mathbf{x}})=\underline{\mathbf{C}} \quad \frac{\mathrm{d}}{\mathrm{d} \underline{\mathbf{x}}}\left(\underline{\mathbf{x}}^{\mathrm{T}} \underline{\mathbf{A}} \underline{\mathbf{x}}\right)=\underline{\mathbf{x}}^{\mathrm{T}}\left(\underline{\mathbf{A}}+\underline{\mathbf{A}}^{\mathrm{T}}\right) \quad \operatorname{grad}(f)=\left(\frac{\mathrm{d} f}{\mathrm{~d} \underline{\mathbf{x}}}\right)^{\mathrm{T}}
$$




\section{Sensorik \& Vorverarbeitungen}

\subsection{Sensorik}

Neben der serienmäßigen Sensorik ist das betrachtete Fahrzeug zur Erkennung anderer Verkehrsteilnehmer mit vier Mid-Range-Radar-Sensoren (MRR) an den Ecken sowie einem Long-Range-Radar-Sensor (LRR) an der Fahrzeugfront ausgestattet. Eine nach vorne gerichtete Monokamera erkennt die Fahrstreifenmarkierungen (Abbildung 2.1).



Abbildung 2.1: Fahrzeugsensorik: Radar blau, Video rot (Reichweiten nicht maßstäblich)

Die Erkennung der Fahrstreifenmarkierungen wird direkt auf der Kamera durchgeführt. Neben den Querablagen und den Winkeln der Fahrstreifenmarkierungen relativ zum eigenen Fahrzeug gibt die Kamera auch deren Krümmungen und Krümmungsänderungen direkt aus.

Es wird davon ausgegangen, dass die Daten der Radarsensoren fusioniert werden und so die Positionen, Geschwindigkeiten und Ausdehnungen der Umfeldobjekte bekannt sind.

\subsection{Lokalisierung im Straßenkoordinatensystem}

Ausgehend von der zur Verfügung stehenden Sensorik liegt es nahe, zur Navigation ein Koordinatensystem zu verwenden, das sich am Verlauf der Straße orientiert. Hierbei verläuft die $\mathrm{x}$-Achse des Straßenkoordinatensystems immer parallel zu den Fahrstreifenmarkierungen, die y-Achse steht senkrecht in Fahrtrichtung links darauf. Jedem Fahrstreifen wird eine Identifikationsnummer (ID) zugeordnet. Die IDs zählen wie auch die y-Koordinate nach links positiv.

Bei der Erzeugung des Straßenkoordinatensystems gibt es zwei Punkte zu berücksichtigen:

- Die Breite der Fahrstreifen verändert sich.

- Fahrstreifenmarkierungen können zeitweise nicht erkannt werden.

Aufgabe der Navigation ist es, beim Fahrstreifenwechsel einen stetigen Verlauf der Fahrzeugposition zu erhalten. Um dies zu erreichen, wird folgender Ansatz verwendet: 
Bei der Initialisierung wird die Mitte des Ego-Fahrstreifens als $p_{\mathrm{rl}, \mathrm{y}}^{\mathrm{r}}=0$ definiert. Dies gilt, solange sich das Ego-Fahrzeug auf diesem Fahrstreifen befindet. Bei einem Fahrstreifenwechsel wird die Mitte des Zielfahrstreifens in Straßenkoordinaten bestimmt und als neuer FixPunkt verwendet, wobei diesem der Wert für $p_{\mathrm{rl}, \mathrm{y}}^{\mathrm{r}}$ zugeordnet wird, der im Moment des Fahrstreifenwechsels gültig war. Wird beispielsweise der rechte Fahrstreifen breiter, während sich das Fahrzeug auf dem linken befindet, kann es vorkommen, dass die Mitte des rechten Fahrstreifens jetzt eine negative y-Koordinate hat (Abbildung 2.2).



Abbildung 2.2: y-Koordinate des Straßenkoordinatensystems beim Fahrstreifenwechsel

Die x-Koordinate des Fahrzeugs wird beim Start des Systems auf $p_{\mathrm{rb}, \mathrm{x}}^{\mathrm{r}}=0$ gesetzt. Im Betrieb wird sie durch Transformation und Integration der Fahrzeuggeschwindigkeit ermittelt. Die bei der Integration auftretenden Fehler sind unkritisch, da die Positionen der Umfeldobjekte relativ zum eigenen Fahrzeug bestimmt werden. Der Absolutwert der x-Koordinate ist daher nicht relevant.

Zusätzlich zu den $\mathrm{x}$ - und y-Koordinaten werden auch die Geschwindigkeit und die Beschleunigung sowie der Verdrehwinkel des Fahrzeugs bezüglich des Straßenkoordinatensystems bestimmt.

Da insbesondere die Nachbarfahrstreifen zeitweise nicht durch die Kamera erkannt werden können, werden einmal erkannte Fahrstreifen für bis zu $20 \mathrm{~s}$ zwischengespeichert. Später sollte ein Fahrstreifenwechsel nur dann begonnen werden, wenn der Fahrstreifen zu diesem Zeitpunkt erkannt wird, oder es andere Hinweise auf seine Existenz gibt. Bei den Fahrversuchen im Rahmen dieser Arbeit wurde dies nicht abgefangen.

Im Verlauf des Projektes hat sich die Navigation im Straßenkoordinatensystem als sehr hilfreich erwiesen. In Fällen, in denen keine einzige Fahrstreifenmarkierung erkannt wird, kann die Querposition jedoch nicht bestimmt werden. Für die Zukunft ist es daher wünschenswert, wenn die Leistungsfähigkeit dieses Moduls noch weiter ausgebaut würde. So wäre es beispielsweise denkbar, einmal erkannte Fahrstreifen in einer lokalen Karte zwischenzuspeichern und dann bei einem Ausfall der Fahrstreifenerkennung mit Hilfe von Inertial- und Fahrzeugsensorik darin bis zur Fahrerübernahme zu navigieren. Zusätzlich könnten die von Kamera und Radar erkannten Umfeldobjekte in solchen Fällen mit zur Ermittlung der Fahrstreifen herangezogen werden. Die oben genannten Punkte könnten auch im Rahmen einer kombinierten Sensordatenfusion erfolgen. 


\subsection{Verarbeitung der Umfeldfahrzeuge}

Die Positionen der erfassten Umfeldfahrzeuge werden in das Straßenkoordinatensystem transformiert. Für jedes Fahrzeug wird für jeden Fahrstreifen eine Belegungswahrscheinlichkeit auf Basis der geschätzten Varianzen aus der Sensorfusion ermittelt. Die Bestimmung dieser Belegungswahrscheinlichkeiten geschah im Vorgängerprojekt für jeden Abtastschritt separat. Zusammen mit der Genauigkeit der Datenerfassung hat dies jedoch nicht zu einer ausreichenden Zuverlässigkeit geführt. Aus diesem Grund wurde eine zusätzliche Filterung und Logik eingeführt, die sicherstellt, dass ein Fahrzeug mindestens einem Fahrstreifen zugeordnet wird und nicht ständig zwischen Fahrstreifen springt (Anhang F.3).

\subsection{Bestimmung geeigneter Lücken}

Auf die ermittelten Belegungswahrscheinlichkeiten der Fahrzeuge wird ein Schwellwert angewendet. Für jeden Fahrstreifen sind daher die Fahrzeuge, die ihn befahren, bekannt. Es wird angenommen, dass ein Fahrzeug den von ihm befahrenen Fahrstreifen über seine gesamte Breite belegt.

Für jeden benachbarten Fahrstreifen werden bis zu drei Lücken bestimmt. Direkt neben dem eigenen Fahrzeug sowie die nächste Lücke davor und dahinter. Lücken zwischen den Fahrzeugen, die kleiner als 5,5 m sind, werden nicht betrachtet. Bei derart unrealistisch kleinen Lücken ist die Wahrscheinlichkeit hoch, dass es sich in Wirklichkeit um einen LKW anstatt zwei getrennter Fahrzeuge handelt. Auf dem Ego-Fahrstreifen wird nur eine Lücke betrachtet, nämlich die zwischen dem vorausfahrenden und dem folgenden Fahrzeug, in der sich das EgoFahrzeug gerade befindet. Insgesamt gibt es also bis zu sieben zu betrachtende Lücken. Für jede dieser Lücken wird in einem Planungsschritt eine Trajektorie berechnet (Abbildung 2.3). Nachgelagert findet dann in jedem Zeitschritt eine Auswahl der am besten geeigneten Trajektorie statt. Diese Auswahl ermöglicht auch einen Abbruch des Fahrstreifenwechsels und ist in Abschnitt 2.6 beschrieben. Einige der Lücken werden jedoch zeitweise deaktiviert, das heißt aus der Betrachtung herausgenommen. Wann dies der Fall ist, wird im Folgenden beschrieben.

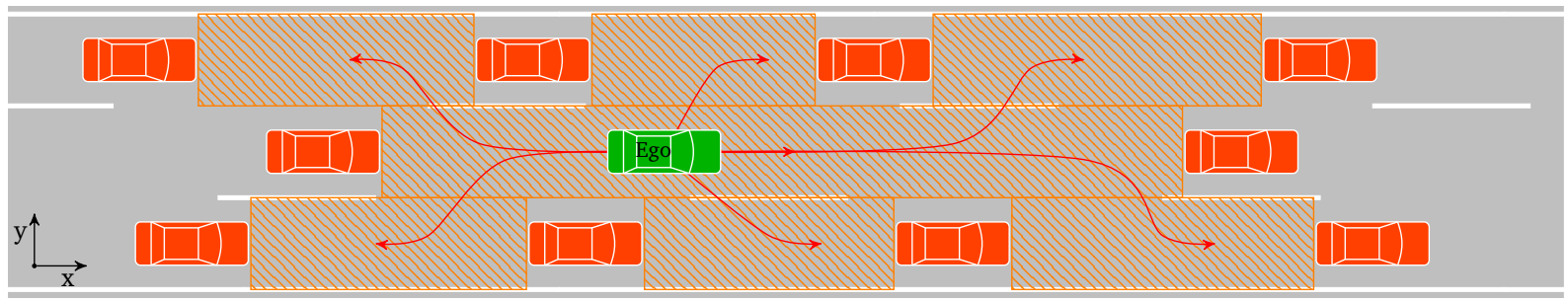

Abbildung 2.3: Der Algorithmus kann in einem Planungsschritt Trajektorien für bis zu sieben Lücken berechnen

Bei einer normalen Fahrt auf der Autobahn wäre das Anfahren der Lücke „links vorne“ sowie „rechts hinten“ in Deutschland problematisch. Beim Anfahren der Lücke „links vorne“ würde 
man ein Fahrzeug rechts überholen. Beim Anfahren der Lücke „rechts hinten“ würde der andere Verkehrsteilnehmer zum Rechtsüberholen genötigt. Es ist relativ wahrscheinlich, dass er bremsen würde und damit das Erreichen der Lücke erschweren würde. Aus diesem Grund sind die Lücken „links vorne“ und „rechts hinten“ normalerweise deaktiviert. Somit werden nur für fünf Lücken Trajektorien berechnet. Auf dem Beschleunigungsstreifen könnte „links vorne“ aber durchaus sinnvoll aktiviert werden.

Darüber hinaus hat sich in Fahrversuchen gezeigt, dass die Sensorreichweite nicht ausreichend ist, um über weit entfernte Lücken zuverlässige Aussagen treffen zu können. Zudem entsprechen diese selten dem Fahrerwunsch. Bei einer Fahrt auf dem mittleren Fahrstreifen und Wechselwunsch nach rechts auf einen Fahrstreifen mit vielen langsameren Fahrzeugen könnte es beispielsweise dazu kommen, dass das System sich immer wieder dazu entscheidet, eine Lücke weiter vorne zu nehmen, da dort kein vorausfahrendes Fahrzeug erkannt wird. Auf diese Weise würde er nie nach rechts wechseln, da immer wieder die Lücke weiter vorne gewählt würde. Aus diesem Grund werden Lücken deaktiviert, die weiter als $30 \mathrm{~m}$ nach vorne bzw. $20 \mathrm{~m}$ nach hinten entfernt sind (Abbildung 2.4).

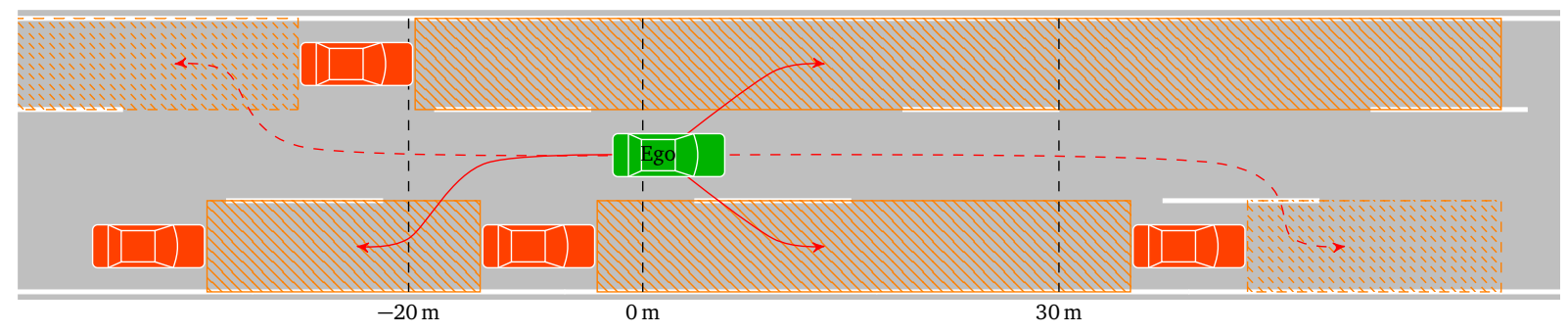

Abbildung 2.4: Weit entfernte Lücken werden deaktiviert

\subsection{Wunschfahrstreifen des Fahrers}

Der Fahrerwunsch ist bei der Auswahl der anzufahrenden Lücke natürlich zu berücksichtigen. Das Fahrzeug soll im Rahmen des Projektes nur dann einen Fahrstreifenwechsel durchführen, wenn dies vom Fahrer gewünscht wird. Erste Ansätze, bei denen nur zu dem Zeitpunkt, zu dem der Blinker gesetzt wird, überprüft wird, ob ein Fahrstreifenwechsel möglich ist, haben sich als nicht besonders leistungsfähig herausgestellt. Denn ist in genau dieser Situation kein Fahrstreifenwechsel möglich, so würde kein Wechsel durchgeführt werden, auch wenn im nächsten Planungsschritt vielleicht eine Trajektorie planbar ist.

Aus diesem Grund wird aus der Blinkerbetätigung ein Wunschfahrstreifen ermittelt, der dann bei der Auswahl der Lücke bzw. Trajektorie berücksichtigt wird. Je nach Situation, in der sich das Fahrzeug befindet, wird bei der Blinkerbetätigung ein Wunschfahrstreifen sowie eine zugehörige Gewichtung bestimmt. Hierdurch kann auch dem vom Fahrer angeforderten Abbruch eines Fahrstreifenwechsels ein höheres Gewicht gegeben werden. Ist der Blinker nicht gesetzt, so liegt der Wunschfahrstreifen auf dem aktuell befahrenen Fahrstreifen. 
Im Versuchsträger, der für diese Arbeit verwendet wurde, muss der Blinker gesetzt werden, um diesen Wunsch an die Trajektorienplanung zu signalisieren. Wenn sich das System entscheidet, den Fahrstreifenwechsel noch nicht durchzuführen, ist dies für andere Verkehrsteilnehmer teilweise irritierend. Um dies zu vermeiden, wäre es denkbar, den Fahrerwunsch auf eine andere Art und Weise zu erfassen. Hierfür gibt es aktuell unterschiedliche Ideen:

- die Nutzung der Tipp-Blinkerfunktion,

- ein Kraft- oder Touch-Sensor im Blinkerhebel,

- ein neuartiges Bedienkonzept, bei dem ggf. auch die Lücke vorwählbar ist.

Bei allen diesen Ansätzen würde das System den Blinker selbstständig setzen, bevor die Querbewegung beginnt.

Der Versuchsträger ist mit einem zweistufigen Blinkerhebel ausgestattet. Die Stufen werden genutzt, um unterschiedliche Prioritäten des Fahrerwechselwunsches zu realisieren. Dies kann beispielsweise sinnvoll sein, wenn der aktuelle Fahrstreifen endet oder eine Ausfahrt noch erreicht werden soll.

\subsection{Auswahl der Ziellücke}

Die Lücken aus Abschnitt 2.4 können über den Fahrstreifen sowie der ID des vorderen und hinteren Fahrzeugs identifiziert werden. Auf diese Weise können sie über mehrere Planungszyklen verfolgt werden. Dies ist unter anderem notwendig, um der Trajektorienplanung den passenden Startwert für die Optimierung aus dem letzten Zyklus bereitstellen zu können. Wenn sich das Umfeld ändert, kann es dazu kommen, dass die ursprüngliche Lücke, definiert durch vorderes und hinteres Fahrzeug, nicht mehr vorhanden ist. In diesem Fall wird die Trajektorie für diesen Fahrstreifen verwendet, die die beste Güte aufweist. Das Gütemaß entspricht dabei dem der Trajektorienplanung (Kapitel 4 \& 5).

Bevor ein Fahrstreifenwechsel auf den Wunschfahrstreifen beginnt, wird geprüft, ob die Wechseltrajektorie bestimmte Kriterien erfüllt. Ist dies der Fall und deren Güte unter Berücksichtigung des Fahrerwunsches besser als die der aktuellen Trajektorie, so beginnt der Fahrstreifenwechsel.

Die Ziellücke wird beibehalten, solange sie existiert. Nur in Fällen, in denen die Lücke nicht mehr vorhanden ist, wird, wie beschrieben, die Trajektorie mit der besten Güte auf dem Zielfahrstreifen verwendet.

Da das System kontinuierlich Trajektorien für verschiedene Lücken plant, ist auch ein Abbruch des Fahrstreifenwechsels möglich. Hierzu wird einfach die Trajektorie verwendet, die wieder zurück in die Startlücke führt. Der Abbruch kann dabei selbstständig vom System oder auf Wunsch des Fahrers ausgelöst werden. Damit ein Wechsel auf die Abbruchtrajektorie erfolgt, muss diese gewisse Sicherheitskriterien erfüllen. Zusätzlich muss die Güte der Abbruchtrajektorie um einen festgelegten Betrag besser sein als die der Wechseltrajektorie. 
Wenn ein Abbruch ausgelöst wurde, beginnt die Planung keinen neuen Fahrstreifenwechsel, bis der Fahrer den Blinker neu setzt. Dies gilt nicht für Fälle, bei denen der Beginn des Fahrstreifenwechsels für den Fahrer noch nicht erkennbar war. Aktuell wird dies auf Basis der Querablage ermittelt.

\subsection{Struktur der Regelung und Trajektorienplanung}

Da sich das Umfeld um das Fahrzeug ständig verändert, ist eine zyklische Neuplanung der Trajektorie notwendig. Im Gegensatz zu einem einfachen Regler ist die Trajektorienplanung vergleichsweise rechenzeitintensiv. Die Regelgüte profitiert jedoch insbesondere im Hinblick auf spontane Störungen von einer möglichst hohen Regelfrequenz.

Bei der klassischen Modellprädiktiven Regelung (MPC) [Graichen, 2017, S. 139 ff.] wird für die Strecke ein, im Sinne des Gütekriteriums, optimaler Stellgrößenverlauf ermittelt. Dieser wird dann bis zur nächsten Optimierung auf die Strecke aufgeschaltet (Abbildung 2.5). Die Regelfrequenz entspricht damit der Berechnungsfrequenz der Modellprädiktiven Regelung. Wenn diese aufgrund der Rechenzeit nicht sehr hoch ausfallen kann, ist eine präzise Kenntnis der Strecke erforderlich. Zwischen zwei Berechnungen kann zudem auf Störungen der Strecke nicht reagiert werden.

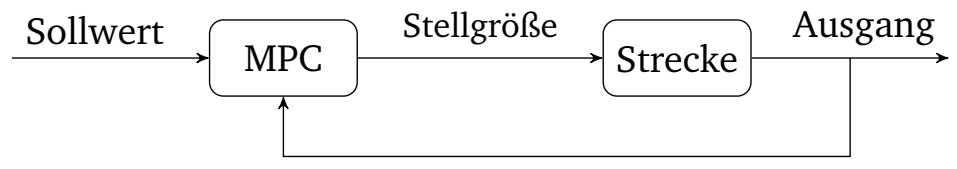

\section{Abbildung 2.5: Blockschaltbild einer klassischen Modellprädiktiven Regelung}

Um den Zielkonflikt zwischen einer langen Planungsdauer und einer hohen Regelfrequenz zu lösen, wird ein Konzept verwendet, das ähnlich zu einer Zwei-Freiheitsgrade-Regelung ist. Die Trajektorienplanung plant eine Trajektorie für ein als Massepunkt (MP) vereinfachtes Fahrzeugmodell. Aufgrund der Einfachheit des Modells lässt sich die Trajektorie leicht analytisch exakt beschreiben. Eine unterlagerte Regelung und Vorsteuerung sorgt nun dafür, dass das Fahrzeug die vorgegebene Trajektorie abfährt. Die Trajektorienplanung wird dabei je nach verwendetem Algorithmus mit $5 \mathrm{~Hz}$ bzw. $10 \mathrm{~Hz}$ Neuplanungsrate betrieben. Die unterlagerte Längs- und Querregelung wird mit einer Regelfrequenz von $50 \mathrm{~Hz}$ betrieben (Abbildungen 2.6 und 2.7). 


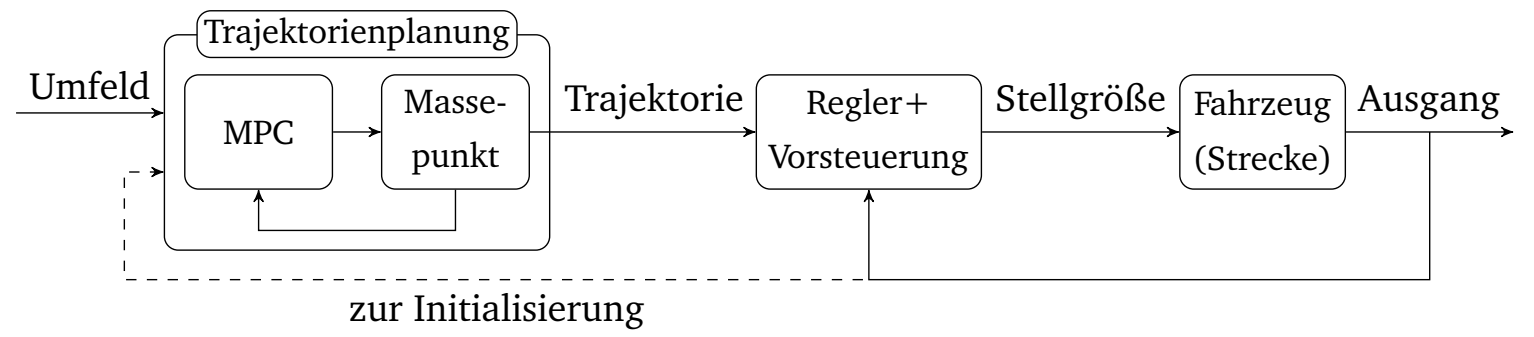

Abbildung 2.6: Trajektorienplanung mit unterlagerter Regelung und Modellprädiktiver Regelung ähnlich zu einer Zwei-Freiheitsgrade-Struktur

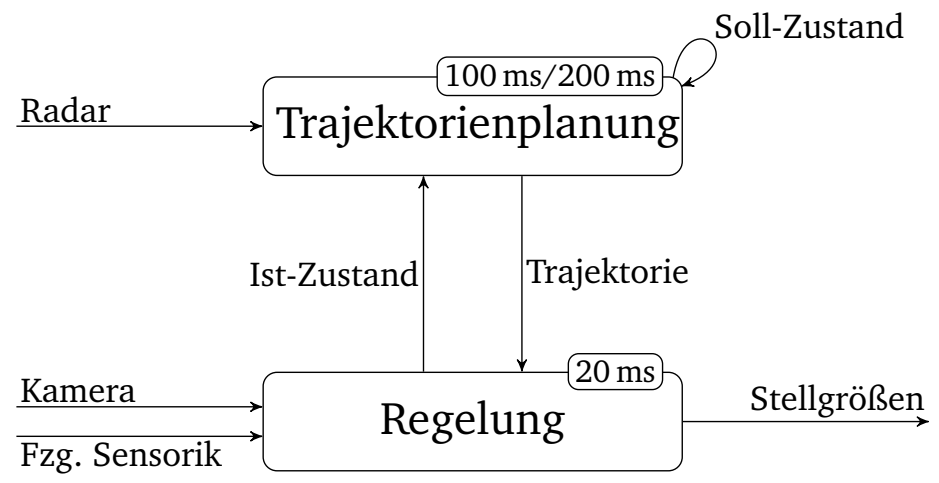

Abbildung 2.7: Vereinfachter Systemaufbau im Versuchsträger. Die Trajektorienplanung findet auf dem Messrechner, die unterlagerte Regelung auf einer Echtzeithardware statt.

\section{Startzustand der Planung}

Die Dauer, die zur Planung einer Trajektorie benötigt wird, kann nicht vernachlässigt werden. Um trotz Neuplanung einen stetigen Verlauf der ausgegebenen Trajektorie zu erhalten, muss diese Zeit berücksichtigt werden. In Abbildung 2.8 ist dies dargestellt. Die Trajektorie, die zum Zeitpunkt $t_{\mathrm{A}}$ geplant wird, muss als Startpunkt den Trajektorienzustand zum Zeitpunkt $t_{\mathrm{B}}$ verwenden. Die Neuplanungszeit $T_{\text {Plan }}$ muss so gewählt werden, dass sie immer größer ist als die Trajektorienberechnungsdauer.

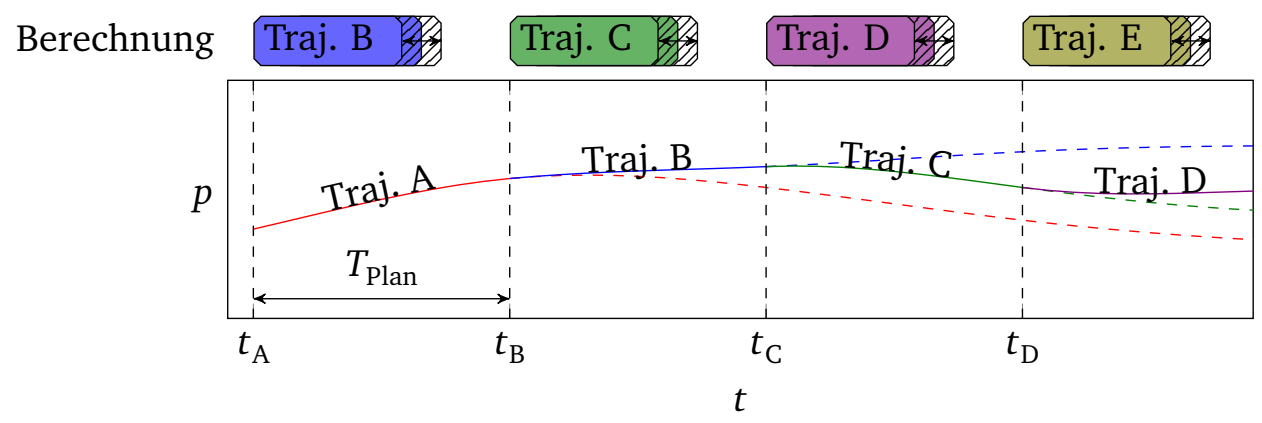

Abbildung 2.8: Die Trajektorie muss im Planungsschritt vor der Gültigkeit berechnet werden

Wie man in Abbildung 2.8 auch sieht, wird als Startzustand für die Trajektorienplanung der zukünftige Zustand der Trajektorie bzw. des Massepunktmodells verwendet. Auf diese Weise 
ist der Verlauf der sich insgesamt ergebenden Trajektorie stetig und weitgehend unabhängig vom Verhalten des realen Fahrzeugs, so dass die in [Werling, 2011, S.6f.] beschriebenen Hundekurven vermieden werden.

Hundekurven entstehen beispielsweise in der Luftfahrt, wenn ein gewisses Ziel angesteuert wird, das Flugzeug jedoch durch den nicht berücksichtigten Wind in eine Richtung abgetrieben wird. Durch diesen Effekt ergibt sich abschließend eine suboptimale Trajektorie, die von der ursprünglich geplanten optimalen Trajektorie abweicht (Abbildung 2.9). Allgemeiner gesprochen entstehen Hundekurven, wenn eine Störgröße nicht in die Trajektorienplanung einbezogen, und der Startzustand der Planung immer wieder an den Istzustand angepasst wird. Durch die Verwendung einer unterlagerten Regelung und Nutzung des Soll- anstatt des Istzustandes als Planungsstartzustand wird dieses Verhalten in dieser Arbeit verhindert.

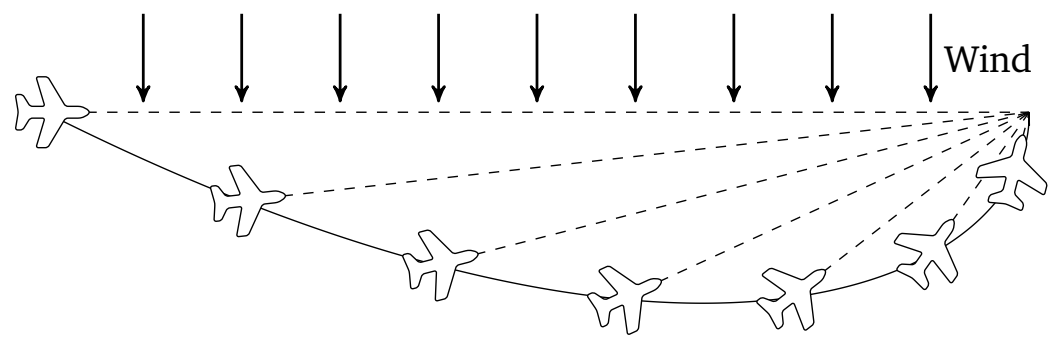

Abbildung 2.9: Eine Hundekurve entsteht z.B. in der Luftfahrt, wenn der Wind bei der Trajektorienplanung nicht berücksichtigt wird und ein Ziel einfach nur direkt angesteuert wird. Vgl. auch [Werling, 2011, S. 6 f.]

In Fällen, in denen noch keine Trajektorie geplant wurde, wird der Startzustand auf Basis des Istzustandes bestimmt. In Längsrichtung ist dies auch dann der Fall, wenn zwar eine Trajektorie geplant wurde, der Fahrer das Fahrzeug jedoch mit dem Gaspedal übersteuert. Wenn sich das reale Fahrzeug deutlich anders als erwartet verhält, wird die Abweichung zwischen Istzustand des Fahrzeugs und Planungsstartzustand begrenzt.

\section{Planungskoordinatensystem}

Würde die Trajektorienplanung direkt im Straßenkoordinatensystem durchgeführt, so bestünde die Gefahr, dass es zu numerischen Problemen kommt, wenn die Zeit und die x-Koordinate im Laufe der Fahrt sehr große Werte annehmen. Aus diesem Grund wird der Ursprung des Straßenkoordinatensystems für die Planung verschoben. Für die Planung der Trajektorie C in Abbildung 2.8 beispielsweise liegt der Nullpunkt in x-Richtung auf der Ist-Position des Fahrzeugs zum Zeitpunkt $t_{\mathrm{B}}$. Der zeitliche Nullpunkt liegt zur Vereinfachung der Berechnungen auf $t_{\mathrm{C}}$. 


\section{Einführung in die Trajektorienplanung}

In diesem Kapitel wird zunächst allgemein auf die Trajektorienplanung eingegangen. Im Anschluss wird die Planung von Fahrstreifenwechseltrajektorien betrachtet sowie Ansätze aus der Literatur vorgestellt. Abschließend wird das Planungsproblem näher analysiert und es wird verdeutlicht, wie die beiden innerhalb dieser Arbeit entwickelten Planungsmethoden das Problem angehen.

\subsection{Trajektorienplanung im Allgemeinen}

Für die Planung eines Fahrstreifenwechsels ist eine Frage von zentraler Bedeutung: „Wie sollen Gas, Bremse und Lenkung des Fahrzeugs angesteuert werden, um einen Fahrstreifenwechsel durchzuführen?" Für derartige Probleme wird häufig ein Optimalsteuerungsansatz gewählt [vStryk, 2014]. Hierzu wird ein Gütekriterium in der Form

$$
J=\underbrace{\vartheta\left(\underline{\mathbf{x}}\left(t_{\mathrm{e}}\right), t_{\mathrm{e}}\right)}_{\text {Mayer-Term }}+\underbrace{\int_{0}^{t_{\mathrm{e}}} \phi(\underline{\mathbf{x}}, \underline{\mathbf{u}}, t) \mathrm{d} t}_{\text {Lagrange-Term }}
$$

aufgestellt. Dabei steht $\underline{\mathbf{x}}$ für den Zustandsvektor des Systems und $\underline{\mathbf{u}}$ für den Eingang. Der Lagrange-Term berücksichtigt eine Bewertungsfunktion $\phi$ entlang der Trajektorie, der MayerTerm die Endsituation zum Zeitpunkt $t_{\mathrm{e}}$. Die Einhaltung der Systemgleichungen

$$
\underline{\dot{\mathbf{x}}}(t)=\underline{\mathbf{f}}(\underline{\mathbf{x}}(t), \underline{\mathbf{u}}(t))
$$

sowie gegebenenfalls weiterer Beschränkungen wird durch Nebenbedingungen berücksichtigt. Durch eine geeignete Wahl von $\underline{\mathbf{u}}(t)$ wird $J$ minimiert. Dadurch erhält man auch die Trajektorie $\underline{\mathbf{x}}(t)$. Auch in dieser Arbeit wird ein derartiger Ansatz verfolgt.

Wird dieses dynamische Optimierungsproblem zyklisch neu gelöst und immer nur der erste Abschnitt des optimalen Stellgrößenverlaufs $\underline{\mathbf{u}}^{*}(t)$ auf das System geschaltet, so ergibt sich eine Modellprädiktive Regelung.

Zur Lösung des dynamischen Optimierungsproblems gibt es unterschiedliche Ansätze. Im Folgenden werden einige Ansätze grob beschrieben, eine detailliertere Übersicht über die Verfahren befindet sich in [Papageorgiou, 2015, S. $387 \mathrm{ff}$.] sowie [Graichen, 2017, S. $82 \mathrm{ff}$.].

Analytisch lassen sich nur sehr einfache dynamische Optimierungsprobleme lösen. Bei komplexeren Problemen ist die Lösung Ergebnis einer iterativen numerischen Optimierung. 
Die häufig erwähnte dynamische Programmierung [Bellman, 1957] ist für das hier betrachtete Problem nur bedingt geeignet. Die Anforderungen an den Komfort und die daraus resultierenden Differenzierbarkeitsanforderungen an die Trajektorie, d. h. die Forderung der Stetigkeit bis zur 2. Ortsableitung, erfordern einen großen Zustandsraum sowie eine feine Diskretisierung desselben. Dadurch wächst der Berechnungsaufwand stark an. Dies hat Bellmann als „Fluch der Dimensionalität" bezeichnet.

Indirekte Methoden liefern zwar sehr genaue Lösungen, haben jedoch nur einen sehr geringen Konvergenzbereich und benötigen eine gute Startlösung. Aus diesem Grund werden sie gerne zur nachträglichen Optimierung der Ergebnisse einer direkten Methode verwendet und hier nicht näher betrachtet. Bei den häufig verwendeten direkten Methoden wird das dynamische Optimierungsproblem auf verschiedene Art und Weise in ein statisches Optimierungsproblem überführt, bei dem die Werte einer endlichen Anzahl an Parametern iterativ bestimmt werden müssen. Im Folgenden sind einige direkte Methoden aus [Papageorgiou, 2015] kurz dargestellt. Bei der Parameteroptimierung werden Eingang $\underline{\mathbf{u}}(t)$ und Trajektorie $\underline{\mathbf{x}}(t)$ durch parametrierbare analytische Funktionen approximiert (z. B. durch Polynome). Passt diese Approximation nicht zu den Systemgleichungen und den Restriktionen, kann die Genauigkeit der Lösung stark leiden.

Beim direkten Einfachschießverfahren wird üblicherweise iterativ ein parametrierbares Eingangssignal erstellt. Anschließend wird die gesamte sich ergebende Systemtrajektorie mithilfe numerischer Integration bestimmt und bewertet. Bei längeren Trajektorien kann je nach System die Konvergenz des Verfahrens eingeschränkt sein.

Beim direkten Mehrfachfachschießverfahren wird die Trajektorie nicht vom Anfang bis zum Ende durch Integration bestimmt, sondern zunächst in viele Segmente unterteilt. Für jedes Segment werden die Anfangsbedingungen zunächst variabel gelassen. Sie sind Teil der Optimierungsvariablen. Innerhalb jedes Segmentes wird der Verlauf durch numerische Integration bestimmt. Mittels Nebenbedingungen an das Optimierungsproblem wird abschließend ein stetiger Verlauf an den Segmentgrenzen gefordert. Die freien Anfangsbedingungen führen zu einer größeren Anzahl an Optimierungsvariablen, wirken sich jedoch positiv auf die Konvergenz der Optimierung aus.

Beim direkten Kollokationsverfahren wird der Verlauf von Eingangs- und Zustandsgrößen in jedem Segment durch Polynomfunktionen approximiert. Es wird gefordert, dass die Zustandsdifferenzialgleichungen (3.2) sowie die Gleichungsnebenbedingungen an einigen diskreten Punkten innerhalb eines jeden Segmentes eingehalten werden. Zudem wird ein stetiger Verlauf der Zustandsgrößen an den Segmentgrenzen gefordert.

Unabhängig vom gewählten direkten Verfahren entsteht immer ein statisches Optimierungsproblem mit einem Gütekriterium, einer gewissen Anzahl an Optimierungsvariablen und einer Reihe von Nebenbedingungen. Dieses kann mit unterschiedlichen Verfahren gelöst werden. Bei einer geringen Anzahl von Optimierungsvariablen ist die Berechnung 
gradientenfrei möglich, beispielsweise mit dem Downhill-Simplex-Algorithmus. Bei größeren Problemen werden Algorithmen verwendet, die den Gradienten der Gütefunktion benötigen. Beispielhaft sei hier auf den SQP-Algorithmus sowie das Quasi-Newton-Verfahren mit BFGS-Approximation verwiesen (SQP: Sequentielle Quadratische Programmierung, BFGS: Broyden-Fletcher-Goldfarb-Shanno). Letzteres wird auch als BFGS-Verfahren oder BFGSAlgorithmus bezeichnet [Broyden, 1970; Fletcher, 1970; Goldfarb, 1970; Shanno, 1970].

\subsection{Trajektorienplanung für Fahrstreifenwechsel}

Betrachtet man nun die Planung einer konkreten Fahrstreifenwechseltrajektorie, so lässt sich das Umfeld zunächst auf vier Fahrzeuge reduzieren. Diese ergeben sich jeweils aus den vorderen und rückwärtigen Fahrzeugen der Start- und Ziellücke (vgl. Abschnitt 2.4). Folgende Abkürzungen werden für die vier Fahrzeuge in dieser Arbeit verwendet (Abbildung 3.1):

- FS: Front Start Lane

- TS: Tail Start Lane

- FD: Front Destination Lane

- TD: Tail Destination Lane

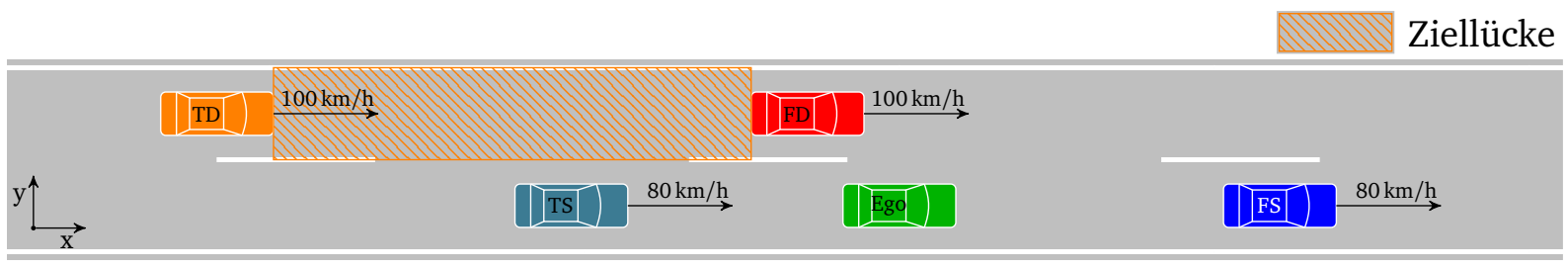

Abbildung 3.1: Beispielszenario mit den Namen der vier Umfeldfahrzeuge für den Fahrstreifenwechsel in eine Ziellücke

Aufgabe der Trajektorienplanung ist es, eine Bewegung zu planen, die das eigene Fahrzeug (Ego) vom Startfahrstreifen in die vorgegebene Lücke auf dem Zielfahrstreifen auf eine komfortable und sichere Art und Weise bewegt. Um die Aufgabe genauer zu analysieren, wird ein Beispielszenario betrachtet, bei dem die Fahrzeuge auf dem rechten Fahrstreifen mit $80 \mathrm{~km} / \mathrm{h}$ und die auf dem linken mit $100 \mathrm{~km} / \mathrm{h}$ fahren. In dieser Arbeit wird der Fokus auf derartige und ähnliche Fahrstreifenwechsel gelegt. Hierbei können die Umfeldfahrzeuge auch sehr verschiedene Geschwindigkeiten besitzen, behalten ihren Fahrstreifen jedoch bei. Komplexere Szenarien, wie endende Fahrstreifen oder fahrstreifenwechselnde Fahrzeuge werden zunächst nicht betrachtet. Einige derartige Szenarien sind in Anhang D aufgeführt.

Das Ego-Fahrzeug soll im Beispielszenario einen Fahrstreifenwechsel vom rechten auf den linken Fahrstreifen durchführen. Die Längspositionen der Umfeldfahrzeuge können in einem $x(t)$ Diagramm aufgetragen werden (Abbildung 3.2). Es ist anzumerken, dass die Fahrzeuge hierbei mit konstanter Geschwindigkeit in die Zukunft prädiziert wurden, der reale Verlauf der Fahrzeugpositionen zum Zeitpunkt der Planung jedoch nicht bekannt ist. 


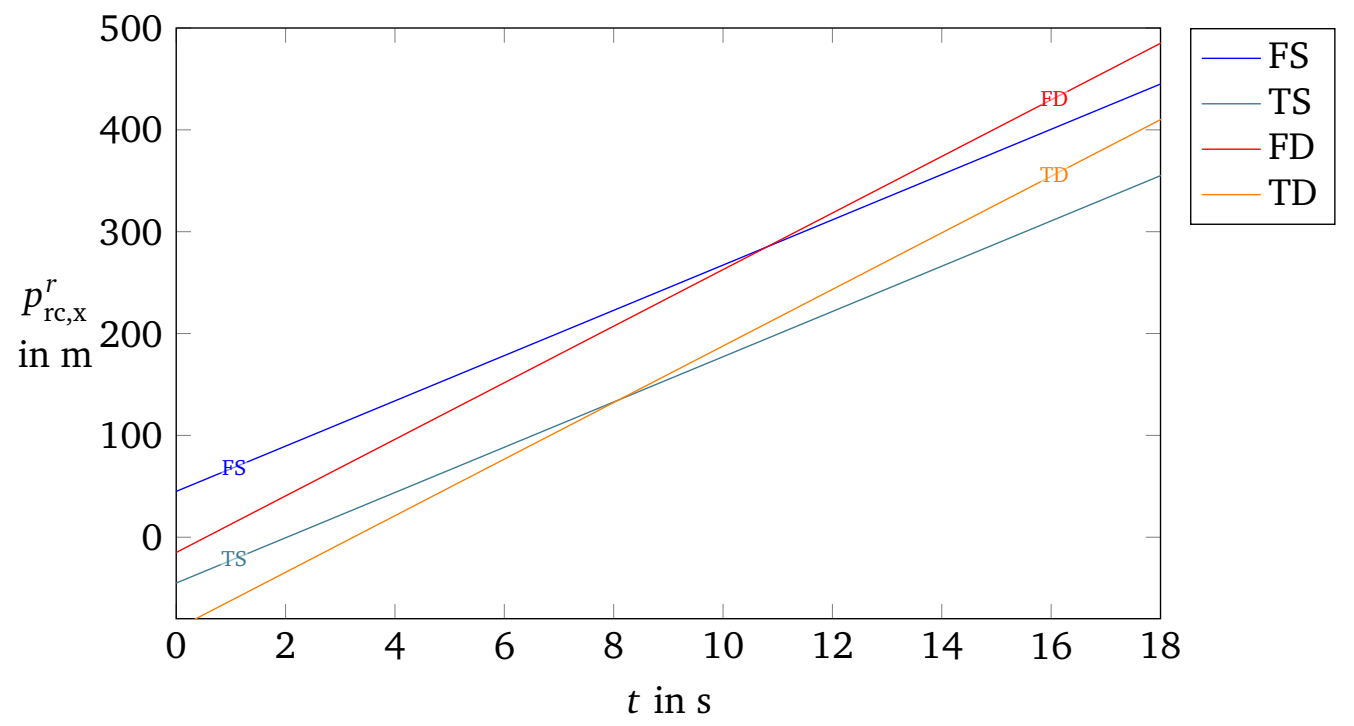

Abbildung 3.2: Längspositionen der Umfeldfahrzeuge im Beispielszenario. Die vorderen Fahrzeuge (FS \& FD) fahren mit $100 \mathrm{~km} / \mathrm{h}$, die rückwärtigen (TS \& TD) mit $80 \mathrm{~km} / \mathrm{h}$

Aufgrund der Tatsache, dass die Abstände zwischen den Fahrzeugen im $x(t)$-Diagramm nur schwer zu erkennen sind, werden alle Positionen im Weiteren relativ zu einem bewegten Beobachter mit konstanter Geschwindigkeit aufgetragen: $x_{\text {rel }}(t)=x(t)-v_{\text {Beobachter }} \cdot t$ (Abbildung 3.3). Dies dient lediglich der besseren Darstellung und hat für die eigentliche Planung keine Bedeutung.

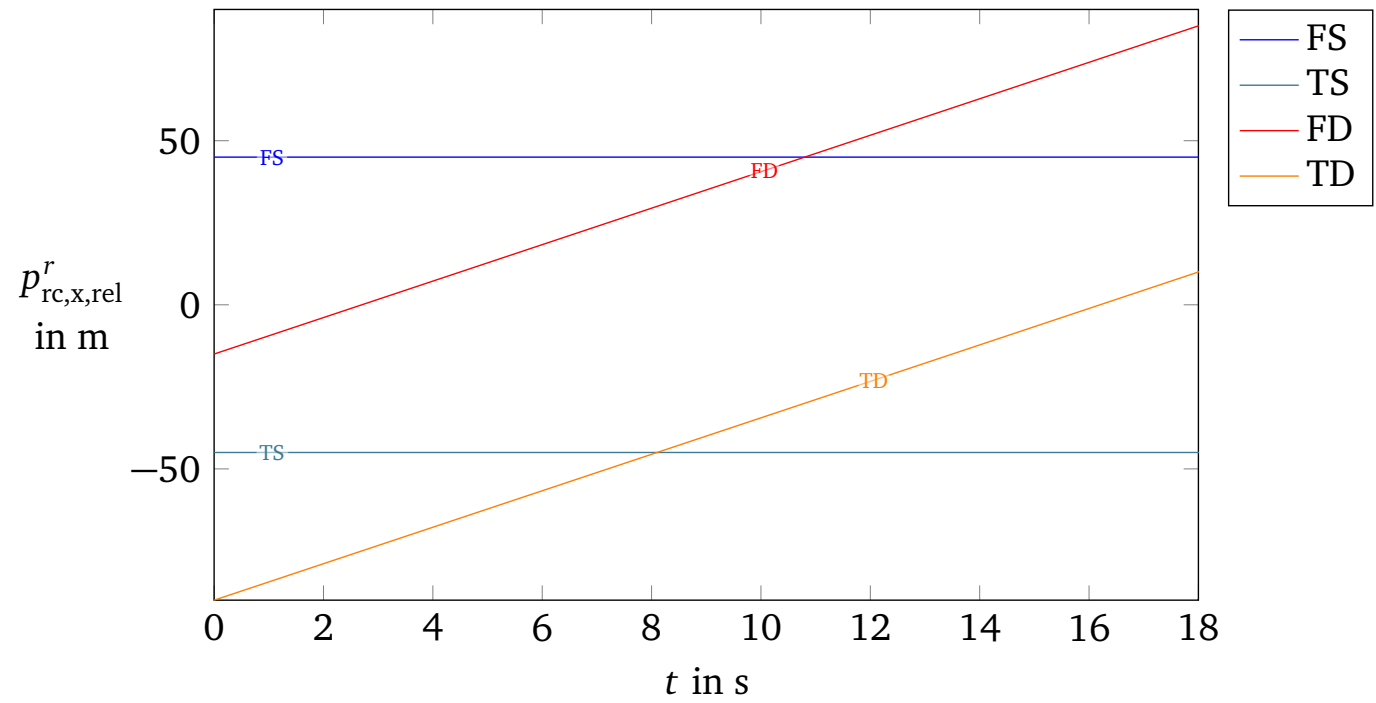

Abbildung 3.3: Längspositionen der Umfeldfahrzeuge relativ zu einem bewegten Beobachter mit $80 \mathrm{~km} / \mathrm{h}$ im Beispielszenario. Die Querbewegung darf nur stattfinden, wenn sich Ego hinter den beiden vorderen Fahrzeugen (FS \& FD) sowie vor den rückwärtigen Fahrzeugen (TS \& TD) befindet. 
Der Fahrstreifenwechsel in Querrichtung muss in dem Zeitbereich stattfinden, in dem sich das eigene Fahrzeug hinter den vorderen Fahrzeugen (FS \& FD) und vor den hinteren Fahrzeugen (TS \& TD) befindet. Dieser Zeitbereich ist abhängig von der gewählten Längstrajektorie.

Bei einfachen Fahrten in statischer Umgebung ist es möglich, zunächst eine Bahn $y(x)$ zu planen. Im Anschluss wird dann z. B. ein Geschwindigkeitsverlauf $v(t)$ geplant. Dies vereinfacht das Problem maßgeblich, ist aber hier, insbesondere aufgrund der zeitabhängigen Umfeldobjektpositionen, so einfach nicht möglich. Längs- und Querbewegung müssen beim Fahrstreifenwechsel aufeinander abgestimmt werden. Aus diesem Grund wird anstatt einer Bahn $y(x)$ eine Trajektorie $x(t), y(t)$ geplant. Je nach gewählter Längstrajektorie $x(t)$ gelten unterschiedliche Beschränkungen an die Quertrajektorie. Dieses Problem bei einer akzeptablen Rechenzeit zu lösen, ist eine der größten Herausforderungen bei der Planung der Fahrstreifenwechseltrajektorie.

\subsection{Verfahren aus der Literatur}

In der Literatur gibt es einige Verfahren, die sich mit der Trajektorienplanung für derartige Probleme beschäftigen. Einige sind speziell auf den Fahrstreifenwechsel zugeschnitten, andere beschäftigen sich allgemeiner mit der kombinierten Längs- und Querplanung. Im Folgenden sind einige Verfahren kurz beschrieben.

\section{Evaluation einer kompletten Trajektorienschar}

In [Werling, 2011, S. $35 \mathrm{ff}$.] wird ein Verfahren beschrieben, bei dem die Trajektorie durch je ein Polynom fünften Grades in Längs- und Querrichtung dargestellt wird. Das Polynom ist hierbei durch seine Anfangs- und Endbedingungen definiert. Durch Variation des Endpunktes wird eine Trajektorienschar generiert. Für jede der Trajektorien wird ein Gütemaß bestimmt und anschließend die Trajektorie mit dem besten Gütemaß ausgewählt. Variiert werden dabei:

- End-Zeit

- End-Querposition

- End-Längsposition

Für jede dieser Größen gibt es eine Anzahl diskreter Werte, die diese annehmen kann. Die Längsgeschwindigkeit und Beschleunigung am Endpunkt ergeben sich hierbei mit Hilfe einer Referenztrajektorie $\xi_{\text {ref }}$ aus der Endposition, der Endzeit sowie dem Umfeld. Aufgrund der diskreten Menge an Endpunkten kann das Verfahren prinzipiell nur eine suboptimale Lösung finden (Abbildung 3.4). Durch die Polynome fünften Grades fällt es schwer, die Maximalbeschleunigung des Fahrzeugs über einen längeren Zeitraum auszunutzen. [Rathgeber, 2016b] beschäftigt sich damit, wie diese Einschränkung durch eine alternative Trajektorienform beseitigt werden kann. 


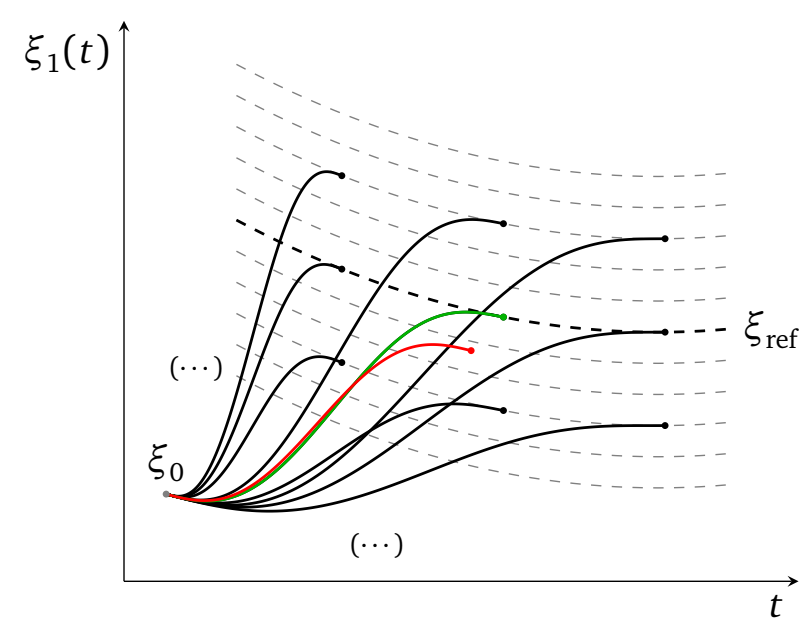

Abbildung 3.4: Trajektorienplanung nach Werling. In schwarz und grün sind die geplanten Trajektorien dargestellt. Die grüne Trajektorie ist diejenige mit dem besten Gütemaß. Die rote Trajektorie ist das theoretische Optimum bei einem kontinuierlich veränderlichen Endpunkt. Bild nach: [Werling, 2011, S. 35]

Dieser Ansatz ist sehr flexibel und sehr robust gegenüber Nebenminima in der Gütefunktion. Allerdings hat er auch einige Einschränkungen. Der benötigte Rechenaufwand steigt sehr stark mit der Anzahl der freien bzw. variierten Endzustände an. Zur Planung eines gesamten Fahrstreifenwechsels ist es notwendig, dass dieser zum aktuellen Zeitpunkt begonnen werden kann. Um den Fahrstreifenwechsel zu einem späteren Zeitpunkt zu beginnen, wäre es denkbar, 2 Polynome hintereinander zu hängen. Hierdurch würde jedoch die Anzahl der Freiheitsgrade wieder stark ansteigen.

\section{Längs- und Quertrajektorie nacheinander bestimmen}

In [Nilsson, 2015] wird ein Verfahren vorgestellt, bei dem für eine diskrete Menge an Startzeitpunkten des Fahrstreifenwechsels je eine Längstrajektorie berechnet wird. Das quadratische Gütemaß berücksichtigt den Komfort, die Kollisionsfreiheit wird durch harte Ungleichungs-Nebenbedingungen berücksichtigt. Die Lösung des Optimierungsproblems wird jeweils durch quadratische Programmierung bestimmt. Es wird der Startzeitpunkt mit dem besten Gütemaß der Längstrajektorie verwendet. Anschließend wird der befahrbare Bereich in Querrichtung bestimmt und ebenfalls mittels quadratischer Programmierung eine Quertrajektorie bestimmt. [Nilsson, 2016] und [Nilsson, 2017] beschäftigen sich damit, wie der Startzeitpunkt sowie die beste Ziellücke vorab bestimmt werden können, um eine mehrfache Berechnung der Längstrajektorie vermeiden zu können. Die Rechenzeit, die auf einem normalen Rechner (Dell Optiplex 990) benötigt wird, ist mit etwa 45 ms angegeben.

Durch die sequentielle Berechnung von Längs- und Quertrajektorie findet kaum eine Rückwirkung der Quer- auf die Längstrajektorie statt. Es wird immer eine feste Mindestzeit $t_{\text {min }}$ für den Fahrstreifenwechsel gefordert. So würde beispielsweise keine schnellere Querbewegung 
durchgeführt werden, um eine bessere Längstrajektorie zu erzielen, beziehungsweise um den Fahrstreifenwechsel überhaupt noch zu ermöglichen. Die harten Grenzen, die an die Längstrajektorie gestellt werden, machen das System vermutlich empfindlich für Störungen. Verschieben sich die Grenzen, z. B. aufgrund von Sensorrauschen oder einem sich verändernden Umfeld, so könnte es dazu kommen, dass keine Trajektorie planbar ist.

\section{Planung mit Polygonzügen}

Im Rahmen der Bertha-Benz-Fahrt im Jahr 2013 wurde ein Verfahren verwendet, bei dem die Trajektorie durch einen Polygonzug repräsentiert wird. Die Ableitungen der Trajektorie werden durch finite Differenzen bestimmt. In das Gütemaß gehen neben dem Komfort auch die Positionsabweichung von einer Referenzbahn sowie die Abweichungen von einem Referenzgeschwindigkeitsvektor ein. Umfeldobjekte werden als zeitabhängige Polygone modelliert. Das eigene Fahrzeug wird durch vier Kreise approximiert. Als Nebenbedingung wird gefordert, dass diese Kreise die Polygone nicht berühren. Hierfür kommt eine spezielle Distanzfunktion zum Einsatz. Abschließend wird das Problem zyklisch alle $500 \mathrm{~ms}$ mit Hilfe sequentieller quadratischer Programmierung (SQP) gelöst. Das Verfahren ist in [Ziegler, 2014] kurz und in [Ziegler, 2015] ausführlicher beschrieben. Fahrstreifenwechsel werden dort jedoch nicht näher betrachtet.

\subsection{Eigene Verfahren}

In diesem Abschnitt wird auf die beiden in dieser Arbeit vorgestellten Verfahren eingegangen. Hierzu wird zunächst das Trajektorienplanungsproblem analysiert und anschließend werden die beiden Lösungsansätze beschrieben.

\section{Analyse des Problems}

Durch die Abhängigkeit der Quertrajektorienbeschränkungen von der Längstrajektorie wird das Problem nichtlinear. Sofern die beiden Planungen nicht wie in [Nilsson, 2015] weitestgehend getrennt gelöst werden, taucht die Nichtlinearität entweder im Gütemaß oder in den Nebenbedingungen auf.

Es wird davon ausgegangen, dass der Komfort eine besonders wichtige Eigenschaft der Trajektorie ist. Die Trajektorie soll daher mehrfach stetig differenzierbar sein. Auf der anderen Seite wird es als nicht ganz so wichtig erachtet, das theoretische Optimum der Gütefunktion zu erreichen. Auch ein normaler Fahrer wird bei zwei Fahrstreifenwechseln in der gleichen Situation gewisse Abweichungen erzeugen.

Um eine möglichst komfortable und vorausschauende Fahrweise zu erreichen, soll die Planung eines Fahrstreifenwechsels schon möglich sein, bevor die Querbewegung eingeleitet werden darf. Dies ermöglicht es z. B. dem Fahrzeug, noch auf dem Startfahrstreifen zu beschleunigen und erst danach den Fahrstreifen zu wechseln. 
Im Vergleich zur Optimierung eines gesamten Rundkurses [Gundlach, 2017] handelt es sich bei einem Fahrstreifenwechsel um ein Problem mit vergleichsweise wenig Optimierungsvariablen. Auf die Verwendung eines Mehrfachschießverfahrens kann daher verzichtet werden.

Da die Funktion später im Fahrzeug umgesetzt werden soll, soll die Trajektorienplanung möglichst flexibel anpassbar sein. Kleine Änderungen an der Gütefunktion sollen nicht zu großen Änderungen im Design oder zu aufwändigen neuen Vorabberechnungen führen. Für die Umsetzung im Fahrzeug ist es von wesentlicher Bedeutung, dass möglichst wenig Rechenzeit benötigt wird.

Es wird davon ausgegangen, dass eine Kollisionsvermeidung allein durch harte Beschränkungen an die Position des eigenen Fahrzeugs ungünstig ist. Diese Beschränkungen werden in der Regel auf Basis einer Prädiktion der Umfeldobjekte bestimmt. Diese entspricht aber selten exakt dem realen Verhalten der Umfeldfahrzeuge. Weicht das Verhalten von der Prädiktion ab, so kann auch mit harten Beschränkungen keine Kollisionsfreiheit mehr garantiert werden. Ebenfalls kann es so dazu kommen, dass sich das eigene Fahrzeug plötzlich in einem Bereich befindet, der nicht zulässig ist oder aus dem keine Trajektorie planbar ist. Dies kann durch Sensorrauschen weiter begünstigt werden. Daher ist es wichtig und entspricht auch dem natürlichen Verhalten, frühzeitig einen gewissen Abstand zu diesen Grenzen einzuhalten.

In den Fahrversuchen hat sich zudem gezeigt, dass es hilfreich ist, auch in kritischen Situationen eine bestmögliche Trajektorie weiter abzufahren, auch wenn diese die Beschränkungen nicht einhält. Auf diese Weise kann die Zeit bis zur Fahrerübernahme sinnvoll genutzt werden, um die Situation zu entspannen.

\section{Ansätze für beide Planungsverfahren}

Die oben erwähnten Punkte wurden in beiden Planungen wie folgt adressiert. Zunächst wurde die Trajektorienplanung möglichst einfach gehalten. Anstatt eines dynamischen Einspurmodells wurde bewusst ein Massepunktmodell (Dreifach-Integrator) verwendet, das sich für die üblichen Autobahngeschwindigkeiten als ausreichend erwiesen hat. Die Verwendung eines Massepunktmodells erlaubt es, die Trajektorie relativ einfach und trotzdem exakt mit Hilfe von Polynomen zu beschreiben. Eine numerische Integration zur Bestimmung des Systemverhaltens ist nicht notwendig. Die linearen Nebenbedingungen, die notwendig sind, um einen stetigen Verlauf der Trajektorie zu erhalten, wurden explizit eingesetzt und so ein großer Teil der Optimierungsvariablen eliminiert. Alle anderen Beschränkungen wurden in die Straffunktion verlagert.

Diese ist bewusst so aufgebaut, dass sie sich im Fahrzeug leicht parametrieren lässt. Zur Einhaltung der Begrenzungen werden äußere Straffunktionen verwendet. Die Gewichtung der relevanten Grenzen wurde dabei so hoch gewählt, dass in der Praxis keine nennenswerten Übertretungen auftauchten. Der Komfort der Trajektorie konnte analytisch für die kontinuier- 
liche Trajektorie bestimmt werden. Die weiteren Strafterme basieren auf der diskretisierten Trajektorie.

Beide Planungen wurden in Matlab implementiert, in der Simulation mit CarMaker getestet und im Fahrzeug umgesetzt. Der dafür notwendige C-Code wurde mit Hilfe des MATLAB-Coders erzeugt. Beide Trajektorienplanungen wurden erfolgreich auf einem Testgelände sowie im realen Straßenverkehr getestet. Ziel der Arbeit war es dabei, nicht ein möglichst serienreifes System zu erstellen, sondern aufzuzeigen, wie eine Trajektorienplanung aussehen kann und welche Effekte zu berücksichtigen sind. Die Untersuchung aller sicherheitstechnischen Detailaspekte ist Aufgabe der nachfolgenden Arbeiten.

Da aktuell keine Informationen über den mittelfristigen Straßenverlauf (Krümmung, Steigung etc.) vorhanden sind, gehen beide Trajektorienplanungen von einem geraden Straßenverlauf aus. Falls diese Informationen später z. B. in einer Karte vorhanden sind, ließen sich beide Planungen entsprechend anpassen.

\section{Drei-Segment-Planung (Kapitel 4)}

Ziel der Drei-Segment-Planung ist es, die Anzahl an Optimierungsvariablen, die durch einen iterativen Optimierungsalgorithmus bestimmt werden müssen, möglichst stark zu reduzieren. Um dies zu erreichen, wird eine spezielle Parametrierung der Trajektorie verwendet.

Zunächst wird aus dem Umfeld der Bereich im $x(t)$-Diagramm bestimmt, in dem beide Fahrstreifen befahren werden dürfen. Dieser Bereich wird als Wechsellücke bezeichnet. Die Trajektorie besteht bei diesem Verfahren aus bis zu drei Segmenten mit variabler Länge:

- Anfahrt zur Wechsellücke auf dem Startfahrstreifen

- Fahrstreifen wechseln

- Weiterfahrt auf dem Zielfahrstreifen

Die Trajektorie besteht in Längsrichtung aus bis zu zwei Polynomen fünften Grades. Im dritten Segment wird die Längsführung durch das ACC übernommen. Als Optimierungsvariablen werden die Durchschnittsgeschwindigkeiten in den ersten beiden Segmenten sowie die Endgeschwindigkeit nach dem zweiten Segment verwendet. Die Quertrajektorie wird passend zur Längstrajektorie so generiert, dass der Fahrstreifenwechsel in der Wechsellücke stattfindet. Die optimalen Werte der Optimierungsvariablen werden ohne Gradienteninformation mit Hilfe eines nichtlinearen Optimierungsalgorithmus (Downhill-Simplex) bestimmt. Die Planung lässt sich am ehesten als Parameteroptimierung sehen, mit den Durchschnitts- und der Endgeschwindigkeit als Parameter.

\section{Spline-Planung (Kapitel 5)}

Bei diesem Ansatz werden mehr Optimierungsvariablen verwendet. Dies resultiert in mehr Freiheiten für die Trajektorie und gestattet es so, die Fahrzeugmöglichkeiten besser ausnutzen 
zu können. Dies ist insbesondere wichtig, um die maximale Beschleunigung über einen längeren Zeitraum aufrecht halten zu können. Um die Rechenzeit im Rahmen zu halten, wird der Gradient der Gütefunktion analytisch bestimmt und dem nichtlinearen Optimierer zur Verfügung gestellt. Als Optimierungsalgorithmus wird das Quasi-Newton-Verfahren mit BFGSApproximation verwendet.

Die Trajektorie besteht bei der Spline-Planung aus 15 Segmenten gleicher zeitlicher Länge. In jedem Segment wird ein Polynom 3. Grades für Längs- und Querrichtung verwendet. Die Optimierungsvariablen sind hierbei die Rucke in den einzelnen Segmenten.

\subsection{Weitere Ansätze}

Neben den beiden oben aufgeführten Planungsmethoden wurden noch weitere Alternativen betrachtet, die jedoch aufgrund ihrer Einschränkungen nicht weiter verfolgt wurden.

\section{Offline-Optimierung}

Im Rahmen einer Offline-Optimierung wäre es möglich, Rechenzeit auf Kosten des Speicherbedarfs einzusparen. Es wird versucht, den Raum möglicher auftretender Szenarien möglichst gut durch offline berechnete Lösungen abzudecken, die dann im Speicher abgelegt werden. Eine grobe Abschätzung hat jedoch ergeben, dass aufgrund der vielen Eingangsvariablen (4 Relativpositionen, 4 Relativgeschwindigkeiten und Ego-Geschwindigkeit) der Speicherbedarf einer Lookup-Tabelle sehr groß ist. Zudem würde eine Änderung der Parametrierung eine langwierige Neuberechnung der ganzen Tabelle nach sich ziehen. Neuronale Netze wurden zum Abspeichern auf Grund des damit verbundenen Fehlerrisikos nicht in Betracht gezogen.

\section{Reglerbasierter Ansatz}

Ein möglicher Minimalansatz wäre die Verwendung einfacher Längs- und Querregler. Der Längsregler sorgt für ein Erreichen der Lücke. Der Querregler wechselt den Fahrstreifen, sobald dies möglich ist. Hierbei stehen unterschiedliche Regler zur Verfügung, zwischen denen umgeschaltet werden kann. Die entstehenden Trajektorien werden vorab simuliert und gegebenenfalls auf einen anderen Regler umgeschaltet. Da vermutet wird, dass dieser Ansatz weniger Szenarien abdecken kann und dabei eventuell unkomfortabler ist, wurde er nicht weiter verfolgt. 


\section{Drei-Segment-Planung}

Für ein möglichst gutes Verständnis wird im nächsten Abschnitt 4.1 erst ein kurzer Überblick über die Drei-Segment-Planung gegeben. Die nachfolgenden Abschnitte gehen anschließend detaillierter auf die einzelnen Teilaspekte ein. Eine ausführlichere mathematische Beschreibung befindet sich im Anhang A.

\subsection{Grundidee}

Ziel des Ansatzes ist es, auch Trajektorien planen zu können, bei denen die Querbewegung erst zu einem Zeitpunkt in der Zukunft einsetzt. Bei der Forderung eines stetigen Beschleunigungsverlaufes wäre es denkbar, für die Längstrajektorie ein einzelnes Polynom fünften Grades zu wählen. Bei diesem ließe sich jedoch der Zeitpunkt, an dem eine gewisse Längsposition erreicht wird, und somit der Fahrstreifenwechsel begonnen werden kann, nur schlecht analytisch berechnen. Aus diesem Grund wird eine Trajektorie aus mehreren Segmenten verwendet.

Es wird ein Algorithmus vorgestellt, bei dem die Längstrajektorie über nur drei Größen parametriert wird. Aus diesen Größen werden dann mit Hilfe des Umfeldes die Orte und zugehörigen Zeitpunkte für den Fahrstreifenwechsel bestimmt. Nach Planung der Längstrajektorie wird eine hierzu passende Quertrajektorie bestimmt. Die Parameter der Längstrajektorie werden durch einen nichtlinearen Optimierungsalgorithmus so variiert, dass sich die optimale Trajektorie im Sinne eines Gütekriteriums ergibt (Abbildung 4.1).

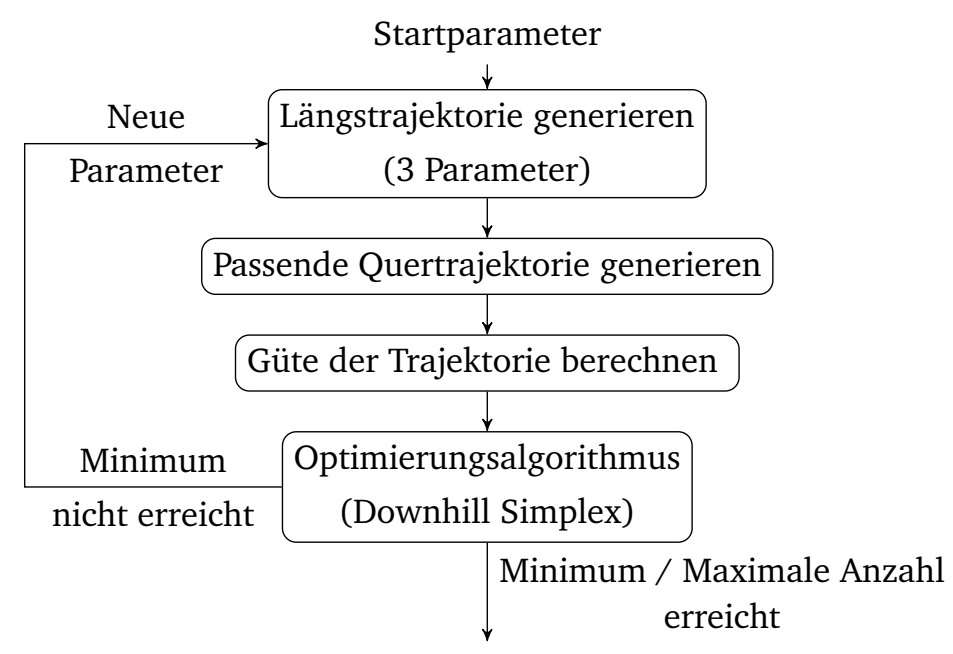

Abbildung 4.1: Bestimmung der optimalen Drei-Segment-Trajektorie 
Es soll eine Trajektorie in Längs- und Querrichtung geplant werden, die das Fahrzeug vom Startfahrstreifen zum Zielfahrstreifen überführt. Hierbei soll das Ego-Fahrzeug stets ausreichend Abstand zu den Umfeldfahrzeugen besitzen.

Die Planung basiert auf folgenden Regeln:

1. Vor und hinter jedem Umfeld-Fahrzeug gibt es einen gewissen Sicherheitsbereich, der nicht befahren werden soll (siehe Abschnitt 4.2).

2. Der Fahrstreifenwechsel muss in einem Bereich stattfinden, in dem sowohl der Start- als auch der Zielfahrstreifen befahren werden dürfen.

3. Nachdem der Fahrstreifenwechsel beendet ist, wird die Längsführung wieder an das ACC übergeben.

Die Längspositionen der Fahrzeuge sowie der Sicherheitsbereichsgrenzen (S...) können über der Zeit $t$ aufgetragen werden (Abbildung 4.2).



Abbildung 4.2: Darstellung der relativen Längspositionen der Fahrzeuge und Sicherheitsbereichsgrenzen über der Zeit

Um die Darstellung übersichtlicher zu gestalten, werden im Folgenden nur die Positionen der Sicherheitsbereichsgrenzen aufgetragen. Der eigentliche Fahrstreifenwechsel darf nur durchgeführt werden, wenn sich das Ego-Fahrzeug vor STD und STS sowie hinter SFD und SFS befindet, es also beide Fahrstreifen befahren darf. Dieser Bereich wird im Folgenden als „Wechsellücke“ bezeichnet (Abbildung 4.3) und ist von den in Abschnitt 2.4 beschriebenen Lücken zu unterscheiden . 




Abbildung 4.3: Darstellung der Wechsellücke zusammen mit den Positionen der Sicherheitsbereichsgrenzen

Der Fahrstreifenwechsel besteht nun aus bis zu drei Phasen bzw. Segmenten (Abbildung 4.4):

1. Längsbewegung zur Wechsellücke (Quer- und Längsführung)

2. Durchführung des eigentlichen Fahrstreifenwechsels in der Wechsellücke (Quer- und Längsführung)

3. Weiterfahrt auf dem Zielfahrstreifen (Querführung, Längsführung durch ACC)

Je nach Situation kann ein Teil der Segmente entfallen (Anhang A.6). Die Trajektorie kann daher aus einem, zwei oder drei Segmenten bestehen.



Abbildung 4.4: Kombination von Quer- und Längstrajektorie für ein Beispielszenario

Die Längstrajektorie wird über die Durchschnittsgeschwindigkeiten in den Segmenten $\left(\bar{v}_{1}, \bar{v}_{2}\right)$ sowie der Geschwindigkeit am Ende von Segment $2\left(v_{\mathrm{e}}\right)$ parametriert. Daraus ergeben sich die Zeitpunkte $t_{1}$ und $t_{2}$ für die Punkte $P_{1}$ und $P_{2}$. Mit Hilfe dieser Zeitpunkte kann eine dazu passende Quertrajektorie geplant werden (Abbildung 4.1, Abbildung 4.4). 


\subsection{Sicherheitsbereiche}

Verwendete Symbole:

Symbol Beschreibung

$p_{\mathrm{rc}, \mathrm{x}, \mathrm{F} . .}^{\mathrm{r}} \quad$ Position der Hinterkante des Fahrzeugs F... relativ zum Straßenkoordinatenursprung

$p_{\text {rc,x,T.. }}^{\mathrm{r}} \quad$ Position der Vorderkante des Fahrzeugs T... relativ zum Straßenkoordinatenursprung

Für die Fahrzeuge (FS, FD, TS, TD) wird je ein Sicherheitsbereich berechnet, dessen Größe sich aus verschiedenen Komponenten zusammensetzt:

- $p_{\text {Safety }}$ : konstanter Anteil

- $t_{\text {Safety }}$ : Zeitlücke (geschwindigkeitsabhängiger Anteil)

- $l_{\mathrm{Ego}, . .}$ : Ausdehnung des Ego-Fahrzeugs vom Ego-Koordinatenursprung zur vorderen bzw. hinteren Kante.

Für die Fahrzeuge auf dem Startfahrstreifen ergibt sich damit z. B.:

$$
\begin{aligned}
& p_{\mathrm{rc}, \mathrm{x}, \mathrm{SFS}}^{\mathrm{r}}=p_{\mathrm{rc}, \mathrm{x}, \mathrm{FS}}^{\mathrm{r}}-\left(v_{\mathrm{rc}, \mathrm{x}, \mathrm{FS}}^{\mathrm{r}} \cdot t_{\text {Safety }}+l_{\mathrm{Ego}, \mathrm{Front}}+p_{\text {Safety }}\right) \\
& p_{\mathrm{rc}, \mathrm{X}, \mathrm{STS}}^{\mathrm{r}}=p_{\mathrm{rc}, \mathrm{x}, \mathrm{TS}}^{\mathrm{r}}+\left(v_{\mathrm{rc}, \mathrm{x}, \mathrm{TS}}^{\mathrm{r}} \cdot t_{\text {Safety }}+l_{\mathrm{Ego}, \mathrm{Tail}}+p_{\text {Safety }}\right)
\end{aligned}
$$

Die Sicherheitsbereichsgrenzen werden nach den Fahrzeugen benannt, wobei ein "S“ für Safety vorangestellt wird (Abbildung 4.5). Die Ausdehnung des eigenen Fahrzeugs wird bei der Bestimmung der Sicherheitsbereiche berücksichtigt. Im Folgenden kann daher das EgoFahrzeug in Längsrichtung als punktförmig angesehen werden, welches die Sicherheitsbereiche nicht verletzen darf. In Querrichtung werden keine Sicherheitsbereiche berechnet. Die Breite des Ego-Fahrzeugs muss daher später berücksichtigt werden.

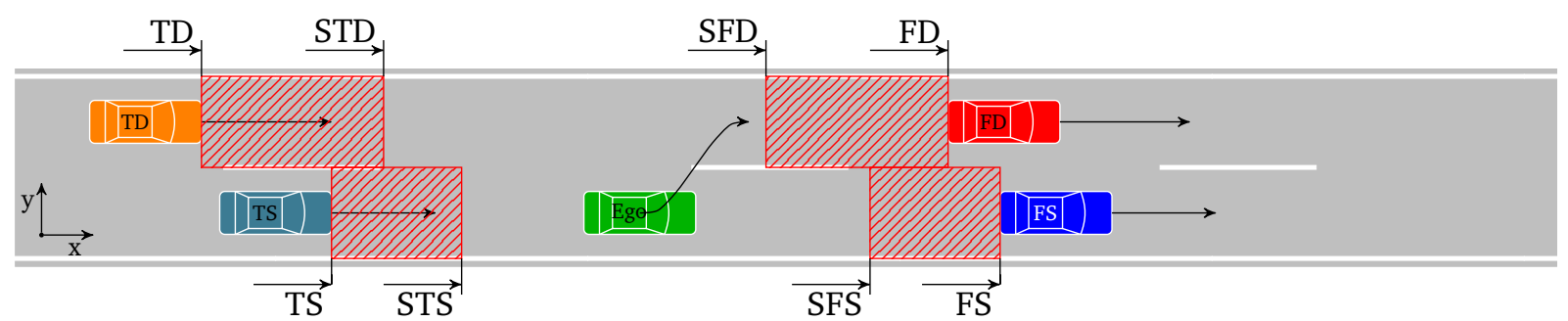

Abbildung 4.5: Darstellung der Sicherheitsbereichsgrenzen für die vier Umfeldfahrzeuge

\subsection{Trajektoriengenerierung}

Die Trajektorienplanung findet nun auf Basis dieser Sicherheitsbereiche statt. Bei einem Wechsel von einem schnellen Fahrstreifen auf einen langsameren kann es notwendig sein, schon auf dem Start-Fahrstreifen zu verzögern, um die Lücke zu erreichen. Bei einer Prädiktion 
des rückwärtigen Verkehrs mit konstanter Geschwindigkeit kann es so allerdings zu einer scheinbaren Kollision mit dem rückwärtigen Fahrzeug auf dem Start-Fahrstreifen kommen. In der Realität ist dies jedoch nicht der Fall, da es Aufgabe des Fahrers des rückwärtigen Fahrzeugs auf dem eigenen Fahrstreifen ist, den Abstand einzuhalten. Daher wird, aus Gründen der Einfachheit, das rückwärtige Fahrzeug auf dem eigenen Fahrstreifen, d. h. je nach Querposition entweder TS oder TD, für die Wechsellückenbetrachtung (Abschnitt 4.1 \& Abschnitt 4.3.1) und die Kollisionsprüfung (Abschnitt 4.5.1) ausgeblendet. Die Bewertung des rückwärtigen Verkehrs auf dem Zielfahrstreifen (Abschnitt 4.5.2) sowie des Endpunktes (Abschnitt 4.5.4) ist hiervon nicht betroffen. Je nach Querposition wird daher entweder TS oder TD ausgeblendet. Zur Vollständigkeit werden im Folgenden weiter beide Fahrzeuge dargestellt. Prinzipiell wäre es auch denkbar, das rückwärtige Fahrzeug auf dem eigenen Fahrstreifen erst auszublenden, nachdem dieser Fahrstreifen einige Sekunden vom eigenen Fahrzeug belegt wurde.

\subsubsection{Generierung der Längstrajektorie}



Abbildung 4.6: Relativpositionen der Umfeldfahrzeuge zusammen mit den Durchschnittsgeschwindigkeiten sowie den Punkten $\mathrm{P}_{0}, \mathrm{P}_{1}$ und $\mathrm{P}_{2}$

Im Vorgängerprojekt wurden Polynome 5. Grades bereits erfolgreich zur Beschreibung komfortabler Quertrajektorien eingesetzt. Sind die Randbedingungen $(p, v, a)$ für Start und Ende jedes Segmentes festgelegt, so ist das Polynom vollständig definiert und stellt einen ruckoptimalen Übergang zwischen den beiden Zuständen dar. Dies wurde unter anderem in [Werling, 2011, S.31f.] gezeigt. Die Vorgabe von Position, Geschwindigkeit und Beschleunigung an Start- und Endpunkt ist wichtig, um einen stetigen Verlauf dieser Größen und somit eine komfortable Trajektorie zu erzielen.

Am Punkt $\mathrm{P}_{0}$ werden neben der Position auch die Geschwindigkeit und Beschleunigung vom Planungs-Startzustand übernommen. Die Lage der Punkte $\mathrm{P}_{1}$ und $\mathrm{P}_{2}$ ist noch frei. 
Um flexibel auf sich bewegende Umfeldobjekte $\mathrm{zu}$ reagieren, werden diese Punkte mit Hilfe der Durchschnittsgeschwindigkeiten $\bar{v}_{1}$ und $\bar{v}_{2}$ in den Segmenten 1 und 2 bestimmt (Abbildung 4.6). Für das Ende des 2. Segmentes wird auch die Endgeschwindigkeit $v_{\mathrm{e}}$ vorgegeben. Die optimalen Werte für die Parameter $\bar{v}_{1}, \bar{v}_{2}$ und $v_{\mathrm{e}}$ werden später durch einen nichtlinearen Optimierungsalgorithmus bestimmt.

Zunächst wird für den Fall, dass keine Umfeldfahrzeuge vorhanden sind, die optimale Fahrstreifenwechseldauer $\Delta t_{\max }$ für einen Fahrstreifenwechsel berechnet (siehe Anhang A.4). Da die Durchschnittsgeschwindigkeit $\bar{v}_{1}$ im 1 . Segment einer Geraden im Diagramm entspricht, kann leicht der Schnittpunkt mit der Wechsellücke bestimmt werden. Dieser Schnittpunkt $\mathrm{P}_{1}\left(t_{1}, p_{1}\right)$ legt Ort und Zeit des Fahrstreifenwechselbeginns fest. Für einen festgelegten Schnittpunkt $\mathrm{P}_{1}$ führt die optimale Fahrstreifenwechseldauer $\Delta t_{\max }$ zu einer vertikalen Geraden (in Abbildung 4.6 bei $t=10 \mathrm{~s}$ ). Anschließend wird eine zweite Gerade vom Punkt $\mathrm{P}_{1}$ mit der Steigung $\bar{v}_{2}$ betrachtet. Der Schnittpunkt dieser Geraden mit der Wechsellücke sowie der vertikalen Geraden wird bestimmt. Der zeitlich frühere Schnittpunkt legt den Punkt $\mathrm{P}_{2}\left(t_{2}, p_{2}\right)$ fest. Wird der Endpunkt durch die Maximaldauer festgelegt, wird zusätzlich noch überprüft, ob sich dieser innerhalb der Wechsellücke befindet. Die Geschwindigkeit am Punkt $\mathrm{P}_{2}$ wird durch $v_{\mathrm{e}}$ festgelegt.

Für den Endpunkt $\mathrm{P}_{2}$ wird schließlich noch die Beschleunigung bestimmt, die das ACC in dieser Situation auf dem Zielfahrstreifen ausgeben wird. Diese Beschleunigung wird für den Endpunkt $\mathrm{P}_{2}$ übernommen, um einen stetigen Übergang der Beschleunigung zu ermöglichen. Da die serienmäßige ACC-Funktion nicht zur Verfügung stand, wird an dieser Stelle das Intelligent-Driver-Model (IDM) [Treiber, 2010, S. 162 f.] als ACC-Ersatz verwendet. Es bestimmt aus Eigengeschwindigkeit, Abstand zum vorausfahrenden Fahrzeug und dessen Relativgeschwindigkeit eine Sollbeschleunigung für das eigene Fahrzeug. Details zum IDM befinden sich in Abschnitt 4.5.2 auf Seite 40.

Mit der Endbeschleunigung sind somit für die Punkte $\mathrm{P}_{0}, \mathrm{P}_{1}$ und $\mathrm{P}_{2}$ folgende Randbedingungen festgelegt:

\begin{tabular}{l|lll} 
& $\mathrm{P}_{0}$ & $\mathrm{P}_{1}$ & $\mathrm{P}_{2}$ \\
\hline$p$ & fix & fix & fix \\
$v$ & fix & stetig & fix \\
$a$ & fix & stetig & fix(weich) ${ }^{1}$
\end{tabular}

$\overline{1}$ In Versuchsfahrten hat sich gezeigt, dass es sinnvoller ist, die Soll-Ist-Abweichung der Beschleunigung am Endpunkt mit hoher Gewichtung ins Gütekriterium zu integrieren, anstatt sie hart in Form einer Nebenbedingung vorzugeben. Ansonsten kommt es bei sehr kurzen Trajektorien und Rauschen bei der Messung der Umfeldfahrzeugpositionen zu Trajektorien, die innerhalb sehr kurzer Zeit große Änderungen der Beschleunigung realisieren. 
Für die Segmente 1 und 2 wird je ein Polynom 5. Grades angesetzt:

$$
\begin{aligned}
& \underline{\mathbf{f}}^{\mathrm{T}}(t)=\left[\begin{array}{llllll}
t^{5} & t^{4} & t^{3} & t^{2} & t & 1
\end{array}\right] \\
& p_{\mathrm{x}}(t)=\left\{\begin{array}{ll}
\underline{\mathbf{f}}^{\mathrm{T}}(t) \cdot \underline{\mathbf{a}} & t_{0} \leq t<t_{1} \\
\underline{\mathbf{f}}^{\mathrm{T}}\left(t-t_{1}\right) \cdot \underline{\mathbf{b}} & t_{1} \leq t<t_{2}
\end{array} \quad \underline{\mathbf{a}}, \underline{\mathbf{b}} \in \mathbb{R}^{6 \times 1}\right.
\end{aligned}
$$

Die verbliebenen Freiheitsgrade werden genutzt, um das Integral über den quadrierten Ruck zu minimieren. Die Polynomkoeffizienten $\underline{\mathbf{a}}$ und $\underline{\mathbf{b}}$ ergeben sich somit aus der Lösung des quadratischen Optimierungsproblems

$$
\underset{\underline{\mathbf{a}}, \underline{\mathbf{b}}}{\arg \min } \int_{t_{0}}^{t_{2}} j_{\mathbf{x}}^{2}(\underline{\mathbf{a}}, \underline{\mathbf{b}}, t) \mathrm{d} t
$$

unter der linearen Nebenbedingung

$$
\underline{\mathrm{C}} \cdot\left[\begin{array}{l}
\underline{\mathbf{a}} \\
\underline{\mathbf{b}}
\end{array}\right]=\underline{\mathrm{d}} .
$$

Die optimalen Parameter $\underline{\mathbf{a}}$ und $\underline{\mathbf{b}}$ können für ein solches Problem durch Lösung eines linearen Gleichungssystems bestimmt werden (siehe Anhang E). Die Generierung der Längstrajektorie sorgt also dafür, dass man sowohl die Längstrajektorie als auch die Zeitpunkte $t_{1}$ und $t_{2}$ in Abhängigkeit der Parameter $\bar{v}_{1}, \bar{v}_{2}$ und $v_{\mathrm{e}}$ erhält. Eine detaillierte Beschreibung der Berechnungen ist in Anhang A.1.1 zu finden. Hierbei kann ein Teil der Parameter durch direkte Berücksichtigung der Nebenbedingungen eliminiert werden. Dort wird auch die Bestimmung der Güte $J_{\text {Lon }}$ beschrieben. Darüber hinaus wird dort auch die Anforderung an die Endbeschleunigung nicht als harte Nebenbedingung formuliert, sondern als quadratischer Strafterm im Gütemaß berücksichtigt.

\subsubsection{Generierung der Quertrajektorie}

Die Quertrajektorie ergibt sich ähnlich wie die Längstrajektorie aus der Minimierung eines Gütefunktionals. Hierfür wird für das 1. und 2. Segment ein Polynom 5. Grades angesetzt. Für das 3. Segment ist, wie sich später zeigen wird, die Verwendung einer MatrixExponentialfunktion sinnvoll. 


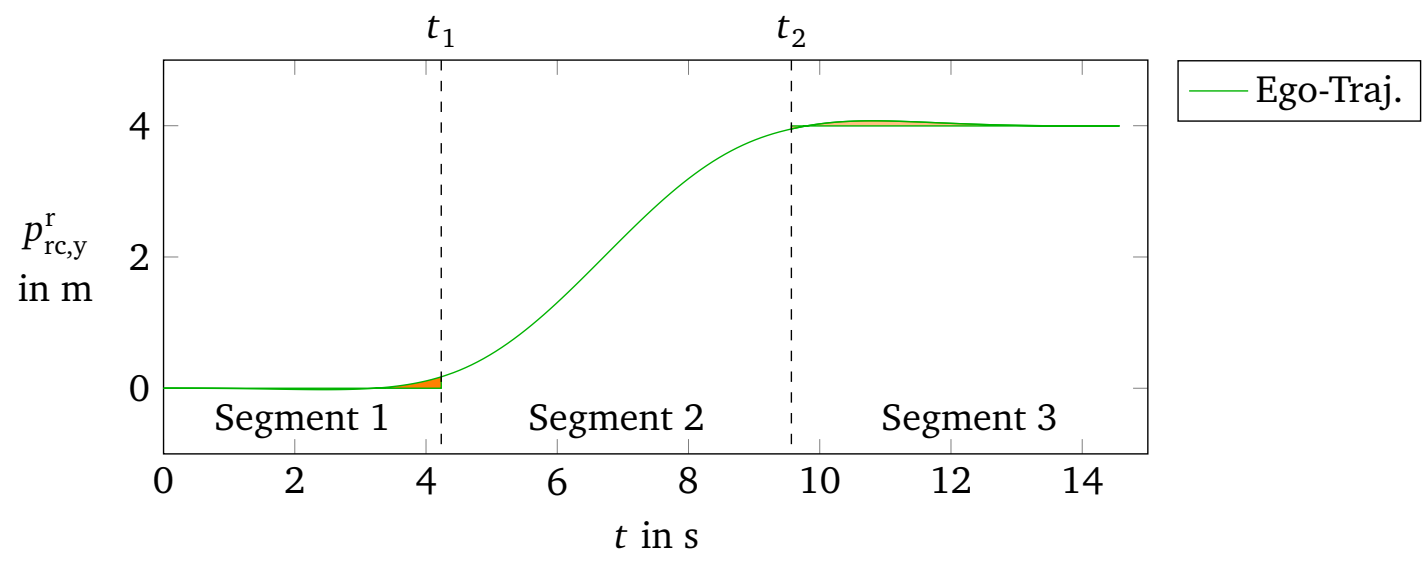

Abbildung 4.7: Beispielhafte Quertrajektorie

Im Kern handelt es sich bei dem Ansatz um eine ruckoptimale Trajektorie im 2. Segment. Es lässt sich zeigen, dass ein Polynom 5. Grades das Gütefunktional $J=\int_{t_{1}}^{t_{2}} j_{\mathrm{y}}^{2} \mathrm{~d} t$ minimiert. Um tolerant gegenüber Rauschen in der Sensorik zu sein, hat es sich jedoch als notwendig erwiesen, geringe Querabweichungen im ersten und dritten Segment zuzulassen. Daher werden die Randbedingungen zu den Zeitpunkten $t_{1}$ und $t_{2}$ nicht fest vorgegeben, sondern bleiben variabel. An diesen Stellen wird lediglich ein stetiger Übergang der Größen $p, v$ und $a$ gefordert. Im 1. Segment gehen die Positionsabweichung vom Startfahrstreifen sowie die Größen $v, a$ und j jeweils in das Gütekriterium ein:

$$
J_{1}=\int_{t_{0}}^{t_{1}}\left(w_{\mathrm{p}, 1}\left(p_{\mathrm{y}}-p_{\mathrm{y}, \text { start }}\right)^{2}+w_{\mathrm{v}, 1} v_{\mathrm{y}}^{2}+w_{\mathrm{a}, 1} a_{\mathrm{y}}^{2}+w_{\mathrm{j}, 1} j_{\mathrm{y}}^{2}\right) \mathrm{d} t
$$

Im 2. Segment basiert das Gütekriterium hauptsächlich auf der Minimierung des quadratischen Rucks. In einigen Parametrierungen der Trajektorie wurden testweise auch Beschleunigung und Geschwindigkeit gewichtet (Anhang A.3):

$$
J_{2}=\int_{t_{1}}^{t_{2}}\left(w_{\mathrm{v}, 2} v_{\mathrm{y}}^{2}+w_{\mathrm{a}, 2} a_{\mathrm{y}}^{2}+w_{\mathrm{j}, 2} j_{\mathrm{y}}^{2}\right) \mathrm{d} t
$$

Im 3. Segment wird das gleiche Gütekriterium wie im 1. Segment verwendet, nur mit dem Unterschied, dass die Abweichung vom Zielfahrstreifen anstatt vom Startfahrstreifen betrachtet wird. Das 3. Segment beginnt bei $t_{2}$ und hat kein definiertes Ende. Daher werden als Integrationsgrenzen $t_{2}$ und $\infty$ verwendet:

$$
J_{3}=\int_{t_{2}}^{\infty}\left(w_{\mathrm{p}, 3}\left(p_{\mathrm{y}}-p_{\mathrm{y}, \text { ziel }}\right)^{2}+w_{\mathrm{v}, 3} v_{\mathrm{y}}^{2}+w_{\mathrm{a}, 3} a_{\mathrm{y}}^{2}+w_{\mathrm{j}, 3} j_{\mathrm{y}}^{2}\right) \mathrm{d} t
$$

Bei der Betrachtung des Gütekriteriums im 3. Segment fällt auf, dass die optimale Lösung dieses Problems durch einen Riccati-Regler erreicht werden kann [Föllinger, 2016, S. 339 ff.]. Die optimale Trajektorie im 3. Segment hat daher die Form:

$$
\underline{\mathbf{v}}(\tau)=\mathrm{e}^{\mathbf{A}_{k} \tau} \underline{\mathbf{v}}_{0} \quad \underline{\mathbf{v}}=\left[\begin{array}{c}
p_{\mathrm{y}} \\
v_{\mathrm{y}} \\
a_{\mathrm{y}}
\end{array}\right]-\left[\begin{array}{c}
p_{\mathrm{y}, \text { Ziel }} \\
0 \\
0
\end{array}\right] \quad \tau=t-t_{2}
$$


Bei vorgegeben Gewichtungen $w_{3, \mathrm{p}}, w_{3, \mathrm{v}}, w_{3, \mathrm{a}}, w_{3, \mathrm{j}}$ lässt sich $J_{3}$ in die Form

$$
J_{3}=\left(\underline{\mathbf{x}}^{\mathrm{T}}\left(t_{2}\right)-\underline{\mathbf{x}}_{3 \mathrm{~s}}^{\mathrm{T}}\right) \overline{\underline{\mathbf{w}}}\left(\underline{\mathbf{x}}\left(t_{2}\right)-\underline{\mathbf{x}}_{3 \mathrm{~s}}\right) \quad \underline{\mathbf{x}}(t)=\left[\begin{array}{c}
p_{\mathrm{y}}(t) \\
v_{\mathrm{y}}(t) \\
a_{\mathrm{y}}(t) \\
j_{\mathrm{y}}(t)
\end{array}\right] \quad \underline{\mathbf{x}}_{3 \mathrm{~s}}=\left[\begin{array}{c}
p_{\mathrm{y}, \text { Ziel }} \\
0 \\
0 \\
0
\end{array}\right]
$$

bringen. Die Berechnung der Matrix $\underline{\underline{W}}$ ist in Anhang A.2 beschrieben.

Somit hängt $J_{3}$ ausschließlich vom Endzustand des 2. Segmentes ab und kann direkt in die Optimierung der ersten beiden Segmente integriert werden. Die optimalen Polynomkoeffizienten a und $\underline{\mathbf{b}}$ im 1. und 2. Segment ergeben sich daher aus

$$
\begin{aligned}
& \underset{\underline{\mathbf{a}}, \underline{\mathbf{b}}}{\arg \min } J_{\mathrm{Lat}}(\underline{\mathbf{a}}, \underline{\mathbf{b}}) \\
= & \underset{\underline{\mathbf{a}}, \underline{\mathbf{b}}}{\arg \min }\left(J_{1}(\underline{\mathbf{a}})+J_{2}(\underline{\mathbf{b}})+J_{3}(\underline{\mathbf{b}})\right) \\
= & \underset{\underline{\mathbf{a}}, \underline{\mathbf{b}}}{\arg \min }\left(\left[\begin{array}{ll}
\underline{\mathbf{a}}^{\mathrm{T}} & \underline{\mathbf{b}}^{\mathrm{T}}
\end{array}\right] \underline{\mathbf{W}}_{\text {Lat, }, 1}\left[\begin{array}{l}
\underline{\mathbf{a}} \\
\underline{\mathbf{b}}
\end{array}\right]+\underline{\mathbf{w}}_{\text {Lat }, 2}^{\mathrm{T}}\left[\begin{array}{l}
\underline{\mathbf{a}} \\
\underline{\mathbf{b}}
\end{array}\right]+w_{\text {Lat }, 3}\right)
\end{aligned}
$$

unter Berücksichtigung linearer Randbedingungen. Diese Randbedingungen wurden im Rahmen der Implementierung genutzt, um einen Teil der Polynomkoeffizienten zu eliminieren. Die optimalen Parameter lassen sich wieder durch Lösen eines linearen Gleichungssystems bestimmen. Die Details zu den Berechnungen und den dafür verwendeten Gewichtungen $\underline{\mathbf{W}}_{\mathrm{Lat}, 1}$, $\underline{\mathbf{w}}_{\text {Lat }, 2}^{\mathrm{T}}$ und $w_{\text {Lat }, 3}$ befinden sich in Anhang A.1.2.

Die für die Berechnung notwendigen Gewichtungen $\underline{\mathbf{W}}_{\text {Lat }, 1}, \underline{\mathbf{w}}_{\text {Lat, } 2}^{\mathrm{T}}$ und $w_{\text {Lat, } 3}$ lassen sich offline in Abhängigkeit der Integrationsgrenzen $t_{1}$ und $t_{2}$ sowie der Start- und Zielfahrstreifenposition bestimmen.

\subsection{Abtastung der Trajektorie}

Neben den Gütetermen aus der Generierung, die im Wesentlichen den Komfort beschreiben, ist eine Bewertung der Trajektorien nach weiteren Kriterien notwendig. Die dafür verwendeten Güteterme haben häufig die Form:

$$
J_{\ldots}=\int_{t_{0}}^{t_{\mathrm{e}}} f_{\ldots}(p(t)) \mathrm{d} t
$$

mit einer abschnittsweise definierten Funktion $f_{\ldots}(p)$ sowie dem Positionsverlauf $p(t)$ der Trajektorie in Form eines Polynoms fünften Grades.

Die analytische Berechnung dieses Integrals wäre recht aufwendig. Grund dafür ist im Wesentlichen die abschnittsweise definierte Funktion $f_{\text {... }}(p)$. Für die Integration bezüglich $t$ 
müssten die Zeitpunkte bestimmt werden, zu denen die Abschnittsgrenzen der Funktion $f_{\text {... }}(p)$ erreicht werden. Dieses Problem entspricht der Nullstellenbestimmung eines Polynoms fünften Grades. Die Lösung dieses Problems ist nach dem Satz von Abel-Ruffini aus dem Jahre 1824 im Allgemeinen nicht durch die vier arithmetischen Grundrechenarten zusammen mit der Wurzelfunktion darstellbar [Bewersdorff, 2013].

Daher wird die Trajektorie für die weitere Bewertung zunächst abgetastet. Hierbei wird für jedes Segment eine konstante Anzahl an Abtastzeitpunkten verwendet. Bei der Abtastung werden die Größen $p_{i}, v_{i}, a_{i}$ und $j_{i}$ sowohl für die Längs- als auch für die Querrichtung ermittelt. In dieser Arbeit werden für jedes der 3 Segmente $n=10$ Abtastzeitpunkte verwendet. Der Abstand der Abtastzeitpunkte ist somit variabel. Dies hat den Vorteil, dass die Abtastzeit bei einem schnellen Fahrstreifenwechsel kürzer ausfällt. Da im letzten Segment nach kurzer Zeit nur noch geringe Bewegungen auftreten, wird als Endzeit $t_{2}+5 \mathrm{~s}$ verwendet. Weil die Längsführung im 3. Segment durch das ACC vorgenommen wird, ist die Trajektorie im 3. Segment nur in Querrichtung definiert.

Das Verhalten des Fahrzeugs zwischen den Abtastpunkten geht nicht in die folgenden Gütefunktionen ein. Daher wurde in Anhang A.7 eine Abschätzung der maximal möglichen Positionsabweichung zwischen den Abtastpunkten durchgeführt. Diese hat ergeben, dass Abtastzeiten bis zu etwa einer Sekunde akzeptabel sind.

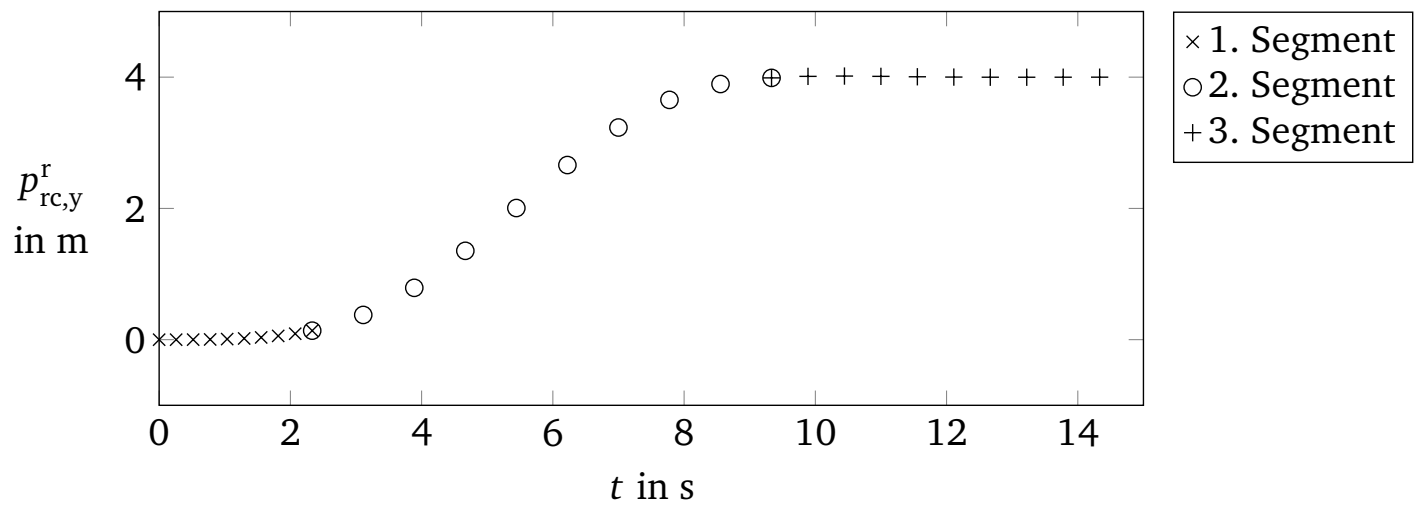

Abbildung 4.8: Beispielhafte Darstellung der Abtastpunkte für eine Quertrajektorie

Viele der im folgenden Abschnitt 4.5 beschriebenen Straffunktionen basieren auf Potentialen, über die integriert wird. Diese Integrale werden auf Basis der Abtastpunkte jeweils numerisch approximiert. Die Abtastzeitpunkte liegen so, dass an Beginn und Ende jedes Segmentes je ein Abtastzeitpunkt liegt (Abbildung 4.8). Bei $n$ Abtastzeitpunkten gibt es somit $n-1$ Zwischenräume in einem Segment. Dies wird bei den Integralapproximationen so berücksichtigt, dass für den ersten und letzten Abtastzeitpunkt eines jeden Segmentes je ein 
halber Zeitschritt angesetzt wird. Bei einer Länge des Segmentes $T$ und einem Abstand zwischen den Abtastzeitpunkten von $\Delta T$ ergibt sich:

$$
\begin{aligned}
\int_{0}^{T} f(t) \mathrm{d} t & \approx \Delta T \cdot\left[\frac{f(0)}{2}+\sum_{i=1}^{n-2}[f(i \cdot \Delta T)]+\frac{f((n-1) \Delta T)}{2}\right] \\
& =\Delta T \cdot \sum_{i=1}^{n-1}\left[\frac{f((i-1) \Delta T)+f(i \cdot \Delta T)}{2}\right]
\end{aligned}
$$

Dies entspricht der Approximation des Integrals durch eine Trapezsumme [Bronstein, 2001, S. 922 f.] und ist in Abbildung 4.9 verdeutlicht. Der Rechenaufwand für die Trapezsumme ist somit nur minimal größer als für eine normale Rechtecksumme und das bei höherer Genauigkeit der Approximation.


Abbildung 4.9: Schematische Darstellung der Integralapproximation. Die rosa Fläche gemäß Gleichung (4.12) ist genauso groß wie die schraffierte Fläche gemäß Gleichung (4.13).

\subsection{Straffunktionen}

Wie in Abschnitt 4.1 beschrieben, wird die Trajektorie über bis zu drei Parameter $\left(\bar{v}_{1}, \bar{v}_{2}\right.$, $v_{\mathrm{e}}$ ) parametriert. Befindet sich das eigene Fahrzeug in der Wechsellücke, müssen nur zwei Segmente geplant werden. In diesem Fall sind nur die Parameter $\bar{v}_{2}$ und $v_{\mathrm{e}}$ zu optimieren. Für den Fall, dass nur noch das letzte Segment zu planen ist, gibt es keine freien Parameter mehr. Die Längsführung wird in diesem Fall durch das ACC übernommen und der verbleibende Querversatz wie beschrieben abgebaut.

Die drei Parameter müssen so gewählt werden, dass die an die Trajektorie gestellten Anforderungen bestmöglich erfüllt werden. Hierzu wird die Trajektorie durch die Straffunktionen nach unterschiedlichen Kriterien bewertet. Im Wesentlichen handelt es sich dabei um die Einhaltung gewisser Beschränkungen bzw. eines ausreichenden Abstands zu ihnen. Die grundlegende Form der Trajektorie ist durch die Generierung schon festgelegt und kann durch die Wahl der Straffunktionen nicht verändert werden. Daher werden die Straffunktionen so gewählt, dass sie sowohl einfach zu berechnen sind, als auch anschaulich in ihrer Wirkung sind. Dies vereinfacht ihre Parametrierung. Der zur Optimierung verwendete Downhill-Simplex-Algorithmus benötigt 
weder einen analytisch berechneten Gradienten noch eine stetig differenzierbare Gütefunktion. Trotzdem muss die Straffunktion so aufgebaut sein, dass an jeder Stelle klar zu erkennen ist, in welche Richtung eine Verbesserung zu erzielen ist.

Die häufig verwendeten abschnittsweise definierten Funktionen sind auf einem Steuergerät effizient durch bedingte Zuweisungen realisierbar. So kann größtenteils auf zeitaufwendige Sprungbefehle verzichtet werden. Zur Reduktion der zur Berechnung notwendigen Operationen wird kein Gesamtpotential $f_{\text {... }}(\underline{\mathbf{x}})$ bestimmt, sondern die Gewichtung auf Ebene der Güteterme



Im Folgenden werden die verwendeten Straffunktionen bzw. Güteterme beschrieben. Die Bewertung der Trajektorie setzt sich zunächst aus den Gütetermen $J_{\text {Lat }}$ und $J_{\text {Lon }}$ aus der Generierung zusammen. Dazu kommen noch folgende weitere Terme:

- $J_{\text {SafetyArea }}$ : Bewertung der Annäherung an den Fahrstreifenrand sowie an die Wechsellückengrenzen in Abhängigkeit der Querposition. (Details: Abschnitt 4.5.1)

- $J_{\text {Traffnfl }}$ : Berücksichtigung der Beeinflussung des rückwärtigen Verkehrs während des Fahrstreifenwechsels. (Details: Abschnitt 4.5.2)

- $J_{\text {EgoDynLim }}$ : Beschränkung der Trajektoriendynamik (Geschwindigkeit, Beschleunigung, Ruck). (Details: Abschnitt 4.5.3)

- $J_{\text {EndEgoBrems }}$ : Bewertung der voraussichtlichen Beschleunigung am Endpunkt für das eigene Fahrzeug. (Details: Abschnitt 4.5.4)

- $J_{\text {EndTDBrems: }}$ Bewertung der voraussichtlichen Beschleunigung am Endpunkt für das rückwärtige Fahrzeug auf dem Zielfahrstreifen. (Details: Abschnitt 4.5.4)

- $J_{\text {ForwardP }}, J_{\text {Forwardv }}$ : Bewertung des Vorankommens basierend auf Endposition und -geschwindigkeit. (Details: Abschnitt 4.5.4)

- Zusätzlich wird die Dauer des 1. und 2. Segmentes $\left(t_{\mathrm{Seg} 1}, t_{\mathrm{Seg} 2}\right)$ bestraft.

Es ergibt sich damit der Güteterm:

$$
\begin{aligned}
J= & w_{\text {Lon }} \cdot J_{\text {Lon }}+w_{\text {SafetyArea }} \cdot J_{\text {SafetyArea }}+w_{\text {TrafInfl }} \cdot J_{\text {Traflnfl }}+ \\
& w_{\text {Lat }} \cdot J_{\text {Lat }}+w_{\text {EndEgoBrems }} \cdot J_{\text {EndEgoBrems }}+w_{\text {EndTDBrems }} \cdot J_{\text {EndTDBrems }}+ \\
& w_{\mathrm{t}, \text { Seg1 } 1} \cdot t_{\text {Seg1 }}+w_{\text {EgoForwardP }} \cdot J_{\text {ForwardP }}+w_{\text {EgoForwardV }} \cdot J_{\text {ForwardV }}+ \\
& w_{\mathrm{t}, \text { Seg2 } 2} \cdot t_{\text {Seg2 }}+w_{\text {EgoDynLim }} \cdot J_{\text {EgoDynLim }}
\end{aligned}
$$

Auf diese Weise werden alle Nebenbedingungen mit in das Gütekriterium integriert. Die verwendeten Gewichtungsparameter sind in Tabelle 4.1 aufgeführt. Wie sich zeigen wird, entsteht ein nichtlineares Optimierungsproblem ohne Nebenbedingungen:

$$
\underset{\bar{v}_{1}, \bar{v}_{2}, v_{\mathrm{e}}}{\arg \min } J\left(\bar{v}_{1}, \bar{v}_{2}, v_{\mathrm{e}}\right)
$$

Im Folgenden werden die einzelnen Terme des Gütekriteriums genauer beschrieben. Die Terme sind dabei zur Reduktion des Berechnungsaufwandes möglichst einfach gehalten. 
Tabelle 4.1: Verwendete Werte der Gewichtungsparameter zur Summation der Güteterme bei der Drei-Segment-Planung

\begin{tabular}{|c|c|c|c|c|c|}
\hline Parameter & Wert & Einheit & Parameter & Wert & Einheit \\
\hline$w_{\text {Lon }}$ & 0,5 & 1 & $w_{\text {EndTDBrems }}$ & 2 & $\mathrm{~s}^{4} / \mathrm{m}^{2}$ \\
\hline$w_{\text {Lat }}$ & 1 & 1 & $w_{\text {EgoForwardP }}$ & 0,015 & $1 / \mathrm{m}$ \\
\hline$w_{\text {SafetyArea }}$ & 1 & $1 / \mathrm{s}$ & $w_{\text {EgoForwardV }}$ & 0,07 & $\mathrm{~s} / \mathrm{m}$ \\
\hline$w_{\text {Traflnfl }}$ & 0,7 & $\mathrm{~s}^{3} / \mathrm{m}^{2}$ & $w_{\mathrm{t}, \mathrm{Seg} 1}$ & 1 & $1 / \mathrm{s}$ \\
\hline$w_{\text {EgoDynLim }}$ & 1 & 1 & $w_{\mathrm{t}, \mathrm{Seg} 2}$ & 0,2 & $1 / \mathrm{s}$ \\
\hline$w_{\text {EndEgoBrems }}$ & 1,5 & $\mathrm{~s}^{4} / \mathrm{m}^{2}$ & & & \\
\hline
\end{tabular}

4.5.1 $J_{\text {SafetyArea }}-$ Bewertung der Annäherung an die Sicherheitsbereiche

Eine wesentliche geforderte Eigenschaft der Trajektorie ist ihre Kollisionsfreiheit. Obwohl die gewählte Parametrierung von sich aus nach Möglichkeit schon Kollisionen vermeidet, können diese nicht grundsätzlich ausgeschlossen werden. Zudem muss von jedem Zwischenpunkt der Trajektorie aus eine Planung durchführbar sein. Befindet sich das Fahrzeug innerhalb der Sicherheitsbereiche, ist dies jedoch nicht garantiert. Um die Planungsmöglichkeit sicherzustellen, sowie Kollisionen zu vermeiden, wird die Annäherung an die Sicherheitsbereiche der Umfeldfahrzeuge sowie eine Annäherung an unbeteiligte Fahrstreifen bestraft.

Der Güteterm $J_{\text {SafetyArea }}$ besteht dazu aus unterschiedlichen Komponenten. Für jede Komponente wird ein zeitabhängiges Potential

$$
\phi_{\text {SafetyArea, }, . .}\left(t, p_{\mathrm{x}}(t), p_{\mathrm{y}}(t)\right)
$$

definiert. $J_{\text {SafetyArea,... }}$ ergibt sich anschließend durch Integration über der Zeit:

$$
J_{\text {SafetyArea, }, . .}=\int_{t_{\mathrm{a}}}^{t_{\mathrm{b}}} \phi_{\text {SafetyArea, } \ldots .}\left(t, p_{\mathrm{x}}(t), p_{\mathrm{y}}(t)\right) \mathrm{d} t
$$

Nach der Berechnung der Funktionswerte an den Abtastpunkten wird das Integral mit der in Abschnitt 4.4 beschriebenen Methode numerisch approximiert. Im Folgenden werden die einzelnen Komponenten betrachtet.

\section{Umfeldfahrzeuge}

Die Trajektorie ist in Längsrichtung nur bis zum Zeitpunkt $t_{2}$ definiert. Anschließend wird die Längsführung durch das ACC übernommen. Die Kollisionsprüfung der Trajektorie mit den Umfeldfahrzeugen findet daher nur innerhalb der ersten beiden Segmente statt. Im letzten Segment wird gefordert, dass sich das Fahrzeug ausschließlich auf dem Zielfahrstreifen befindet, sodass Kollisionen dort durch das ACC vermieden werden. 
Betrachtet wird zunächst beispielhaft das Fahrzeug FD. Hierzu werden als Zwischengrößen die Eindringtiefen $\tilde{x}$ und $\tilde{y}$ gemäß Abbildung 4.10 definiert. Bei $\tilde{x}=0$ befindet sich der Koordinatenursprung des Fahrzeugs gerade $4 \mathrm{~m}$ von dem Sicherheitsbereich entfernt. Für $\tilde{y}=0$ befindet sich die Kante des Fahrzeugs $20 \mathrm{~cm}$ von der Fahrstreifenmarkierung entfernt.

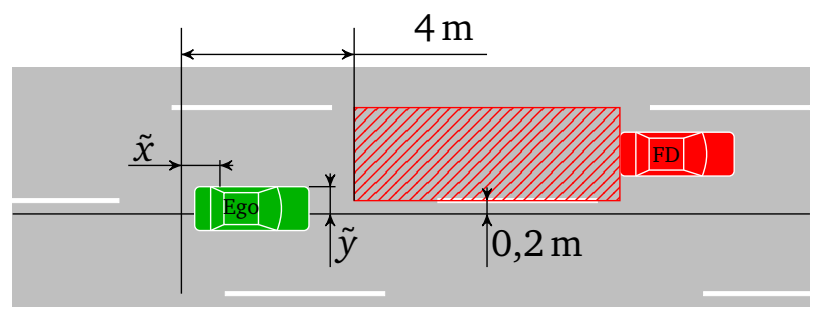

Abbildung 4.10: Definition von $\tilde{x}$ und $\tilde{y}$, dargestellt für positive Werte von $\tilde{x}$ und $\tilde{y}$.

Die Annäherung an das Umfeldfahrzeug wird mit folgender Potentialfunktion ${ }^{2}$ bestraft:

$$
\phi_{\text {SafetyArea,FD }}(\tilde{x}, \tilde{y})= \begin{cases}\tilde{x}^{2} \cdot\left(100 \tilde{y}+\tilde{y}^{2}\right) \cdot 10^{-4} & \tilde{x}>0 \wedge \tilde{y}>0 \\ 0 & \tilde{x} \leq 0 \vee \tilde{y} \leq 0\end{cases}
$$

Die Funktion steigt in x-Richtung langsam an, in y-Richtung sehr steil (Abbildung 4.11). Dies wurde so realisiert, da für die Querführung eine wesentlich höhere Genauigkeit notwendig ist als für die Längsführung.



Abbildung 4.11: Straffunktion $\phi_{\text {SafetyArea,FD }}(\tilde{x}, \tilde{y})$ für die Annäherung an das Fahrzeug FD

Da die Position der Umfeldfahrzeuge und somit auch die Größen $\tilde{x}$ und $\tilde{y}$ zeitabhängig sind, ergibt sich eine zeitabhängige Potentialfunktion $\phi_{\text {SafetyArea,FD }}\left(t, p_{\mathrm{x}}, p_{\mathrm{y}}\right)$. Das Gesamtpotential

2 Die Funktion $\phi_{\text {SafetyArea,... }}$ ist für $\tilde{y}=0$ nicht differenzierbar. Streng mathematisch gesehen handelt es sich daher nicht um ein Potential. Da dies jedoch hier von untergeordneter Bedeutung ist, und zudem einige Potentialeigenschaften weiterhin gelten, wird aus Konsistenzgründen trotzdem von einem Potential gesprochen. 
$\phi_{\text {SafetyArea,Cars }}$ ergibt sich aus der Summe der Potentiale für die vier Umfeldfahrzeuge. Durch Integration von 0 bis $t_{2}$ erhält man den Strafterm:

$$
J_{\text {SafetyArea,Cars }}=\int_{0}^{t_{2}} \underbrace{\mathrm{d} t}_{\phi_{\text {SafetyArea,Cars }}\left[\sum_{c \in\{\mathrm{FS}, \mathrm{FD}, \mathrm{TS}, \mathrm{TD}\}} \phi_{\text {SafetyArea }, \mathrm{c}}\left(t, p_{\mathrm{x}}(t), p_{\mathrm{y}}(t)\right)\right]}
$$

\section{Fahrstreifen}

Neben der Kollisionsfreiheit mit den vier Umfeldfahrzeugen muss sichergestellt werden, dass das eigene Fahrzeug keine unbeteiligten Fahrstreifen befährt. Bestraft wird ein drohendes Verlassen der zulässigen Fahrstreifen. Die Annäherung $\tilde{y}$ an einen unbeteiligten Fahrstreifen wird dazu gemäß Abbildung 4.12 definiert. Für $\tilde{y}=0$ befindet sich die Kante des Fahrzeugs $20 \mathrm{~cm}$ vor der Fahrstreifenmarkierung.

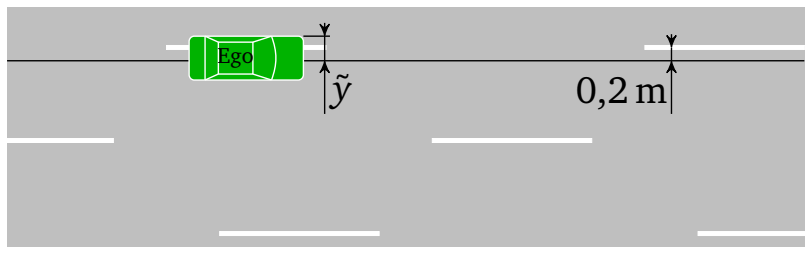

Abbildung 4.12: Definition der Annäherung $\tilde{y}$ an einen unbeteiligten Fahrstreifen

Die Straffunktion besteht aus einem linearen und einem quadratischen Term, sodass sie von Anfang an steil ansteigt (Abbildung 4.13):

$$
f(\tilde{y})= \begin{cases}10 \tilde{y}+10 \tilde{y}^{2} & \tilde{y}>0 \\ 0 & \tilde{y} \leq 0\end{cases}
$$

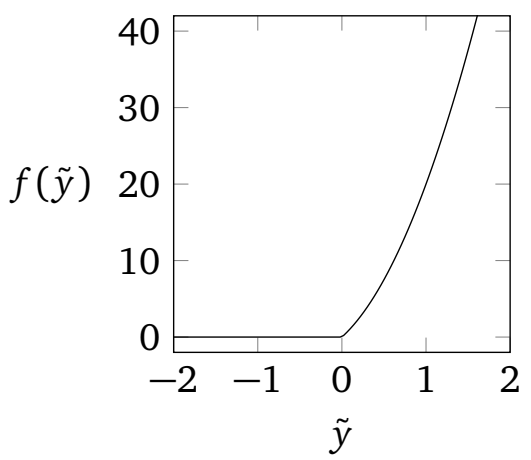

Abbildung 4.13: Straffunktion für die Annäherung an einen Fahrstreifenrand

Das Potential setzt sich grundsätzlich aus der Straffunktion für den linken und rechten Rand des befahrbaren Bereiches zusammen:

$$
\phi_{\text {SafetyArea,Lane, }, . .}=f_{\text {links }}\left(\tilde{y}_{\text {links }}\right)+f_{\text {rechts }}\left(\tilde{y}_{\text {rechts }}\right)
$$


Innerhalb der ersten beiden Segmente dürfen Start- und Zielfahrstreifen befahren werden. Das Potential $\phi_{\text {SafetyArea,Lane,1,2 }}$ setzt sich daher aus dem linken und rechten Rand von Startund Zielfahrstreifen zusammen. Für das Potential $\phi_{\text {SafetyArea,Lane,3 }}$ des dritten Segments wird gefordert, dass das Fahrzeug ausschließlich den Zielfahrstreifen befährt.

\section{Gesamtpotential}

Die Potentiale für Umfeldfahrzeuge und Fahrstreifen lassen sich in den ersten beiden Segmenten zu einem Gesamtpotential $\phi_{\text {SafetyArea,1,2 }}$ kombinieren. Zur Verdeutlichung wird hier ein Szenario betrachtet, in dem zwei schnellere Fahrzeuge auf dem linken Fahrstreifen zwei langsame auf dem rechten Fahrstreifen überholen (Abbildung 4.14). Für jeden Zeitpunkt $t$ gibt es eine Funktion $\phi_{t}\left(p_{\mathrm{x}}, p_{\mathrm{y}}\right)$, die festlegt, wie „gut“ der jeweilige Punkt $\mathrm{P}\left(p_{\mathrm{x}}, p_{\mathrm{y}}\right)$ ist.

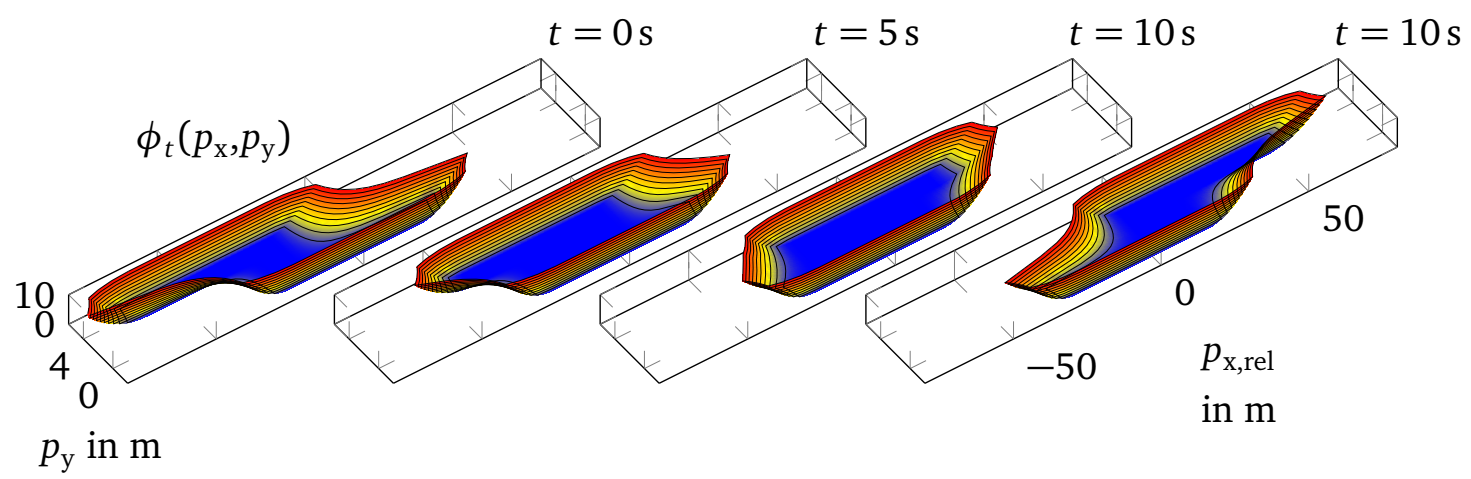

Abbildung 4.14: Beispiel für ein Potential $\phi_{t}\left(p_{\mathrm{x}}, p_{\mathrm{y}}\right)$ zur Bestimmung von $J_{\text {SafetyArea, } 1,2}$.

Um $J_{\text {SafetyArea }}$ zu erhalten, müssen alle Einzelterme addiert werden:

$$
\begin{aligned}
J_{\text {SafetyArea }} & =\underbrace{\int_{0}^{t_{2}} \phi_{\text {SafetyArea,Cars }}\left(t, p_{\mathrm{x}}, p_{\mathrm{y}}\right) \mathrm{d} t}_{J_{\text {SafetyArea,Cars }}}+\underbrace{\int_{0}^{t_{2}} \phi_{\text {SafetyArea,Lane, } 1,2}\left(t, p_{\mathrm{y}}\right) \mathrm{d} t}_{J_{\text {SafetyArea,Lane, }, 2}} \\
& +\underbrace{\int_{t_{2}}^{t_{2}+5 \mathrm{~s}} \phi_{\text {SafetyArea,Lane,3 }}\left(t, p_{\mathrm{y}}\right) \mathrm{d} t}_{J_{\text {SafetyArea,Lane,3 }}} t
\end{aligned}
$$

\subsection{2 $J_{\text {TrafInfl }}$ - Bewertung des Einflusses auf den rückwärtigen Verkehr}

Neben der im letzten Abschnitt betrachteten Kollisionsvermeidung ist es ebenfalls wichtig, einen ausreichenden Abstand zu den anderen Fahrzeugen einzuhalten. Hierbei ist insbesondere der rückwärtige Verkehr auf dem Zielfahrstreifen von Bedeutung. Auch wenn die Trajektorie kollisionsfrei ist, würde der Fahrer dieses Fahrzeugs bei einem zu geringen Abstand verzögern. Dies soll nach Möglichkeit verhindert werden. Je nach Situation lässt sich dies, gerade wenn der Fahrstreifenwechsel schon begonnen wurde, nicht ganz vermeiden. Insbesondere wenn 
das vorausfahrende Fahrzeug während des Fahrstreifenwechsels bremst, muss ein vernünftiger Kompromiss gefunden werden. Kurz gesprochen sorgt dieser Term für eine ausreichende Beschleunigung des eigenen Fahrzeugs während des Fahrstreifenwechsels, falls dies notwendig ist.

Es wird daher davon ausgegangen, dass der rückwärtige Verkehr grundsätzlich in begrenztem Maß zur Kooperation bereit ist, sofern er die Wechselabsicht erkennt. Bei einem ersten Ansatz wurde keine Kooperation berücksichtigt. Dies führte dazu, dass eigentlich unkritische Situationen zu stark bestraft wurden und teilweise ein Abbruch des Fahrstreifenwechsels erfolgte.

Für die Modellierung des rückwärtigen Verkehrs wird das Intelligent-Driver-Model (IDM) [Treiber, 2010, S.162f.] verwendet. Dies ist ein verhältnismäßig einfaches Modell zur Beschreibung eines menschlichen Fahrers. Ausgang dieses Modells ist eine Soll-Beschleunigung, die basierend auf der Eigengeschwindigkeit $v$, der Differenzgeschwindigkeit $\Delta v$ und dem Abstand $s$ bestimmt wird:

[Treiber, 2010, S. 162 f.]:

$$
\begin{aligned}
a_{\mathrm{IDM}} & =a_{\max }\left[1-\left(\frac{v}{v_{0}}\right)^{\delta}-\left(\frac{s^{*}(v, \Delta v)}{s}\right)^{2}\right] \\
s^{*}(v, \Delta v) & =s_{0}+\max \left(0, v T+\frac{v \Delta v}{2 \sqrt{a_{\max } b}}\right)
\end{aligned}
$$

Verwendete Symbole:

$$
\begin{array}{cl}
a_{\mathrm{IDM}} & \text { ausgegebene Beschleunigung } \\
v & \text { aktuelle Eigengeschwindigkeit } \\
\Delta v & \text { Differenzgeschwindigkeit } v-v_{\text {Front }} \\
s & \text { aktueller Abstand } p_{\text {Front }}-p \\
s^{*} & \text { Wunschabstand } \\
v_{0} & \text { Wunschgeschwindigkeit } \\
s_{0} & \text { Minimalabstand } \\
T & \text { Folgezeit } \\
a_{\text {max }} & \text { Maximalbeschleunigung } \\
b & \text { komfortable Verzögerung } \\
\delta & \text { Beschleunigungsexponent }
\end{array}
$$


Es wird angenommen, dass sich der Fahrer des rückwärtigen Fahrzeugs TD so verhält, als ob sein Fahrzeug mit einem ACC auf Basis des IDM ausgestattet ist. Dieses ACC reagiert, sobald das Ego-Fahrzeug den Zielfahrstreifen befährt. Positive Beschleunigungen werden nicht betrachtet:

$$
a_{\mathrm{TD}, \text { unlimitiert }}(t)= \begin{cases}\min \left(0, a_{\mathrm{IDM}, \mathrm{TD} \leftrightarrow \mathrm{Eg} 0}(t)\right) & \text { Überdeckung mit Zielfahrstreifen } \\ 0 & \text { sonst }\end{cases}
$$

Es wird angenommen, dass der Fahrer des rückwärtigen Fahrzeugs davon ausgeht, dass er bei einem Fahrstreifenwechsel nicht alleine für die Einhaltung des Sicherheitsabstandes sorgen muss. Daher wird für die Prädiktion des rückwärtigen Fahrzeugs nur die Hälfte dieser Verzögerung und maximal $-a_{\mathrm{TD}, \text { MaxMitwirkung }}=1,0 \mathrm{~m} / \mathrm{s}^{2}$ angenommen:

$$
a_{\mathrm{TD}, \text { Prädiktion }}(t)=\max \left(a_{\mathrm{TD}, \text { MaxMitwirkung }}, \frac{a_{\mathrm{TD}, \text { unlimitiert }}(t)}{2}\right)
$$

Die Integration zu Geschwindigkeit und Position geschieht dann mittels des Euler-vorwärtsVerfahrens [Bronstein, 2001, S. 927]. Diese Prädiktion wird für jeden Trajektorienkandidaten neu durchgeführt.

Das Maß $J_{\text {TrafInfl }}$ für die Beeinflussung des rückwärtigen Verkehrs ergibt sich schließlich aus den Werten, die das fiktive ACC während des Fahrstreifenwechsels ausgegeben hat:

$$
J_{\text {TrafInfl }}=\int_{t_{0}}^{t_{2}} a_{\mathrm{TD}, \text { unlimitiert }}^{2} \mathrm{~d} t
$$

\subsection{3 $J_{\text {EgoDynLim }}$ - Beschränkung der Trajektoriendynamik}

Die Beschränkung der Trajektoriendynamik hat verschiedene Ziele. Da als Fahrzeugmodell eine einfache Integratorkette verwendet wird, darf die Trajektoriendynamik insbesondere in Querrichtung nicht $\mathrm{zu}$ hoch sein. Zudem sorgt die Beschränkung für eine Einhaltung der Stellgrößen- und Stellratenbegrenzungen der Aktoren. Abschließend stellt der Term sicher, dass die Trajektorien gewisse Komfortgrenzen einhalten.

Um diese Ziele zu erreichen, existieren Ober- und Untergrenzen für die Größen $v$, $a$ und j. Eine Überschreitung der Grenzen wird quadratisch bestraft. Die jeweiligen Werte zu den Abtastpunkten werden dazu mit nichtlinearen Kennlinien bewertet. Abbildung 4.15 zeigt am Beispiel der Längsbeschleunigung die Kennlinie $f_{\mathrm{a}, \mathrm{Lon}}(a)$ :

$$
f_{\mathrm{a}, \mathrm{Lon}}(a)=\left\{\begin{array}{lr}
k_{\mathrm{Lon}, \mathrm{a}, \max } \cdot\left(a-a_{\mathrm{Lon}, \max }\right)^{2} & a_{\mathrm{Lon}, \max }<a \\
0 & a_{\mathrm{Lon}, \min } \leq a \leq a_{\mathrm{Lon}, \max } \\
k_{\mathrm{Lon}, \mathrm{a}, \min } \cdot\left(a_{\mathrm{Lon}, \min }-a\right)^{2} & a<a_{\mathrm{Lon}, \min }
\end{array}\right.
$$




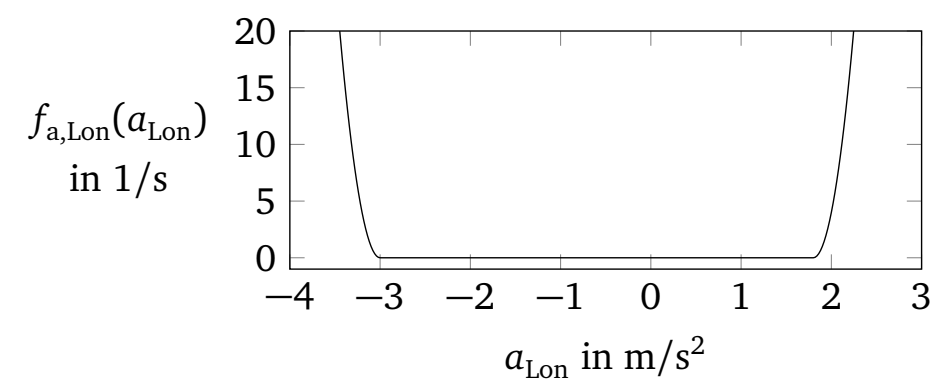

Abbildung 4.15: Beispielhafter Verlauf der Funktion $f_{\mathrm{a}, \mathrm{Lon}}(a)$ bei $a_{\max }=1,8 \mathrm{~m} / \mathrm{s}^{2}$

Tabelle 4.2: Übersicht der Gewichtungsfaktoren für die unterschiedlichen Größen

\begin{tabular}{cccr} 
Größe & $k_{\ldots, \min }$ & $k_{\ldots, \text { max }}$ & Einheit \\
\hline$k_{\text {Lat, }}$ & 100 & 100 & $\mathrm{~s} / \mathrm{m}^{2}$ \\
$k_{\text {Lat,a }}$ & 100 & 100 & $\mathrm{~s}^{3} / \mathrm{m}^{2}$ \\
$k_{\text {Lat,j }}$ & 100 & 100 & $\mathrm{~s}^{5} / \mathrm{m}^{2}$ \\
$k_{\text {Lon,v }}$ & 10 & 0,01 & $\mathrm{~s} / \mathrm{m}^{2}$ \\
$k_{\text {Lon,a }}$ & 100 & 100 & $\mathrm{~s}^{3} / \mathrm{m}^{2}$ \\
$k_{\text {Lon,j }}$ & 10 & 10 & $\mathrm{~s}^{5} / \mathrm{m}^{2}$
\end{tabular}

Die Gewichtungsfaktoren $k_{\ldots}$ sind in Tabelle 4.2 aufgeführt. Je nach Gewichtungsfaktor wirkt die Grenze unterschiedlich hart. So kann es zum Beispiel durch Veränderung der Setzgeschwindigkeit dazu kommen, dass das Fahrzeug schneller als die Setzgeschwindigkeit fährt. In diesem Fall soll das Fahrzeug zügig verzögern, jedoch keine Notbremsung durchführen. Die maximal mögliche Verzögerung soll in diesem Fall auf keinen Fall überschritten werden. Diese Prioritäten lassen sich gut über die unterschiedlichen Größenordnungen der Gewichtungsfaktoren realisieren.

Für die unterschiedlichen Größen $v, a$ und $j$ in Längs- und Querrichtung existieren unterschiedliche Beschränkungen, die in Tabelle 4.3 aufgeführt sind.

Tabelle 4.3: Übersicht der Beschränkungen für die unterschiedlichen Größen

\begin{tabular}{cccl} 
Größe & $\min$ & $\max$ & Einheit \\
\hline$v_{\text {Lat }}$ & -2 & 2 & $\mathrm{~m} / \mathrm{s}$ \\
$a_{\text {Lat }}$ & -1 & 1 & $\mathrm{~m} / \mathrm{s}^{2}$ \\
$j_{\text {Lat }}$ & -1 & 1 & $\mathrm{~m} / \mathrm{s}^{3}$ \\
$v_{\text {Lon }}$ & 2 & $v_{\text {ACC,Setz }}$ & $\mathrm{m} / \mathrm{s}$ \\
$a_{\text {Lon }}$ & -3 & $a_{\max }(v)$ & $\mathrm{m} / \mathrm{s}^{2}$ \\
$j_{\text {Lon }}$ & -3 & 3 & $\mathrm{~m} / \mathrm{s}^{3}$
\end{tabular}

Die maximale Längsbeschleunigung $a_{\max }(v)$ ergibt sich aus der Motorleistung, der Fahrzeugmasse und den Fahrwiderständen sowie einer konstanten maximalen Beschleunigung unter 
der Annahme $v_{\text {Lon }} \approx v$ (Abbildung 4.16). Im Rahmen der Identifizierung der Längsaktorik (Anhang F.2.1) konnte der Verlauf von $a_{\max }(v)$ experimentell validiert werden. Aktuell wird weiterhin angenommen, dass eine gerade Fahrbahn ohne Steigung vorliegt. Ist dies nicht der Fall, so müssten die Daten z. B. aus einer Karte entnommen und berücksichtigt werden.

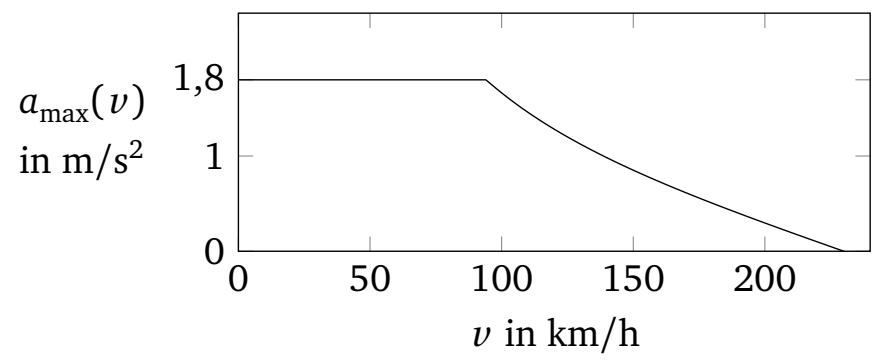

\section{Abbildung 4.16: Maximale Längsbeschleunigung in Abhängigkeit der Geschwindigkeit}

$J_{\text {EgoDynLim }}$ setzt sich aus Termen für Geschwindigkeit, Beschleunigung und Ruck in Längs- und Querrichtung zusammen:

$$
\begin{aligned}
J_{\text {EgoDynLim }}= & J_{\text {EgoDynLim,v,Lon }}+J_{\text {EgoDynLim,a,Lon }}+J_{\text {EgoDynLim,j,Lon }}+ \\
& J_{\text {EgoDynLim,v,Lat }}+J_{\text {EgoDynLim,a,Lat }}+J_{\text {EgoDynLim,j,Lat }}
\end{aligned}
$$

Die Strafterme für die Beschleunigung lauten beispielsweise:

$$
J_{\text {EgoDynLim,a,Lon }}=\int_{t_{0}}^{t_{2}}\left[f_{\text {a,Lon }}\left(a_{\text {Lon }}(t)\right)\right] \mathrm{d} t \quad J_{\text {EgoDynLim,a,Lat }}=\int_{t_{0}}^{t_{2}+5 \mathrm{~s}}\left[f_{\text {a,Lat }}\left(a_{\text {Lat }}(t)\right)\right] \mathrm{d} t
$$

Die anderen Strafterme sind analog dazu aufgebaut. Für die Berechnung wird wieder die in Abschnitt 4.4 beschriebene Approximation des Integrals verwendet.

\subsubsection{Bewertung der Endsituation in Längsrichtung}

Die sinnvolle Bewertung des Endpunktes ist wichtig, da die anderen Terme nur den Verlauf während des Fahrstreifenwechsels berücksichtigen. Verletzt die Trajektorie die Sicherheitsbereiche nicht, führt aber danach zu einer Kollision, so kann das nur durch eine Endpunktbewertung erkannt werden. Dies ist insbesondere wichtig, da die Trajektorie keine feste zeitliche Länge besitzt und unter Umständen auch sehr kurz sein kann.

\section{Vorankommen}

Dieser Term sorgt für eine Belohnung einer großen Endposition und einer Endgeschwindigkeit, die sich möglichst nahe an der ACC-Setzgeschwindigkeit befindet. Ohne diesen Term gäbe es keinen Grund für das Fahrzeug zu beschleunigen. 
Da die Trajektorie eine variable Länge besitzt, muss die Endposition auch in Bezug zur Endzeit gesehen werden. Aus diesem Grund wird in dieser Arbeit die Positions-Differenz zu einer Konstantfahrt mit $v_{\text {Endp,avg }}=100 \mathrm{~km} / \mathrm{h}$ ermittelt:

$$
J_{\text {ForwardP }}=-\left[p_{\mathrm{x}}\left(t_{2}\right)-\left[p_{\mathrm{x}}\left(t_{0}\right)+v_{\text {Endp,avg }} \cdot\left(t_{2}-t_{0}\right)\right]\right]
$$

Bei der direkten Verwendung der Endposition würden lange Trajektorien immer belohnt und der Fahrstreifenwechsel unnötig in die Länge gezogen. Das negative Vorzeichen sorgt für eine Belohnung einer möglichst großen Endposition.

Für die Bewertung der Endgeschwindigkeit wird der Betrag der Abweichung von der ACCSetzgeschwindigkeit verwendet:

$$
J_{\text {ForwardV }}=\left|v_{\mathrm{x}}\left(t_{2}\right)-v_{\mathrm{ACC}, \text { Setz }}\right|
$$

\section{Ego-Situation}

Es soll vermieden werden, dass das eigene Fahrzeug am Endpunkt der Trajektorie bremsen muss, um den gewünschten Abstand zum vorausfahrenden Fahrzeug (FD) einzuhalten. Um dies in die Bewertung einfließen zu lassen, wird für die Situation am Punkt $\mathrm{P}_{2}$ (Abbildung 4.6) für das eigene Fahrzeug die Sollbeschleunigung $a_{\mathrm{IDM}, \mathrm{Eg} \leftrightarrow \leftrightarrow \mathrm{FD}}$ einer fiktiven ACC-Funktion berechnet. Hierfür wird das Intelligent-Driver-Model [Treiber, 2010, S. 162 f.] verwendet. Im Gegensatz zur Festlegung des Endpunktes wird die maximal mögliche Verzögerung hier nicht begrenzt. Positive Beschleunigungen werden nicht berücksichtigt. Die ermittelte Verzögerung geht quadratisch in das Gütemaß ein:

$$
J_{\text {EndEgoBrems }}=\left[\min \left(a_{\mathrm{IDM}, \mathrm{Ego} \leftrightarrow \mathrm{FD}}, 0\right)\right]^{2}
$$

\section{Situation von TD}

Natürlich sollte auch das rückwärtige Fahrzeug TD am Endpunkt nicht bremsen müssen. Daher wird, ausgehend vom prädizierten Verlauf von TD aus der Ermittlung von $J_{\text {Traflnfl}}$, für die Endsituation analog zu $J_{\text {EndEgoBrems }}$ die Sollbeschleunigung für das rückwärtige Fahrzeug auf dem Zielfahrstreifen in Bezug zum Ego-Fahrzeug berechnet und als Gütekriterium verwendet:

$$
J_{\text {EndTDBrems }}=\left[\min \left(a_{\mathrm{IDM}, \mathrm{TD} \leftrightarrow \mathrm{Eg} g}, 0\right)\right]^{2}
$$

\subsection{Bestimmung der optimalen Trajektorie}

Nachdem die Trajektorie auf Basis von unterschiedlichsten Kriterien bewertet wurde, werden die einzelnen Strafterme gemäß Gleichung (4.14) gewichtet und addiert, wie dies in 
Abschnitt 4.5 beschrieben wurde. Somit liegt schließlich für die Trajektorie eine Gesamtgüte in Abhängigkeit der Parameter $\bar{v}_{1}, \bar{v}_{2}$ sowie $v_{\mathrm{e}}$ vor. Alle Nebenbedingungen wurden entweder eingesetzt oder mit in das Gütekriterium integriert. Auf einen stetigen Gradientenverlauf wurde zugunsten einer einfachen Berechnung der Gütefunktion verzichtet.

Um die zur Optimierung der Gütefunktion notwendigen Parameter $\bar{v}_{1}, \bar{v}_{2}$ sowie $v_{\mathrm{e}}$ zu bestimmen, wurde das Downhill-Simplex-Verfahren eingesetzt, das ohne Gradienteninformation auskommt [Nelder, 1965], [Lagarias, 1998]. Hierzu wurde die in MATLAB enthaltende Funktion fminsearch so portiert, dass sie auch innerhalb einer Embedded-Matlab-Function verwendet werden kann. Dadurch ist es möglich, Code für die Zielhardware zu erzeugen.

Während der Fahrtests wurde die Anzahl der Funktionsauswertungen auf 400 begrenzt. Teilweise wurden auch Fahrstreifenwechsel mit nur 30 berechneten Trajektorienkandidaten durchgeführt. Hierbei kam es subjektiv zu keinem Komfortverlust, allerdings kann es bei schnellen Änderungen in der Umfeldsituation dazu kommen, dass die optimale Trajektorie erst nach einigen Planungsschritten gefunden wird.

Als Startwerte $\left(\bar{v}_{1}, \bar{v}_{2}, v_{\mathrm{e}}\right)$ für die Optimierung im aktuellen Planungsschritt werden die Werte aus dem vorherigen Planungsschritt übernommen. In Anhang A.5 wird beschrieben, wie die Startwerte ggf. angepasst werden müssen, um sicherzustellen, dass die Wechsellücke erreicht wird und somit eine Trajektorie planbar ist.

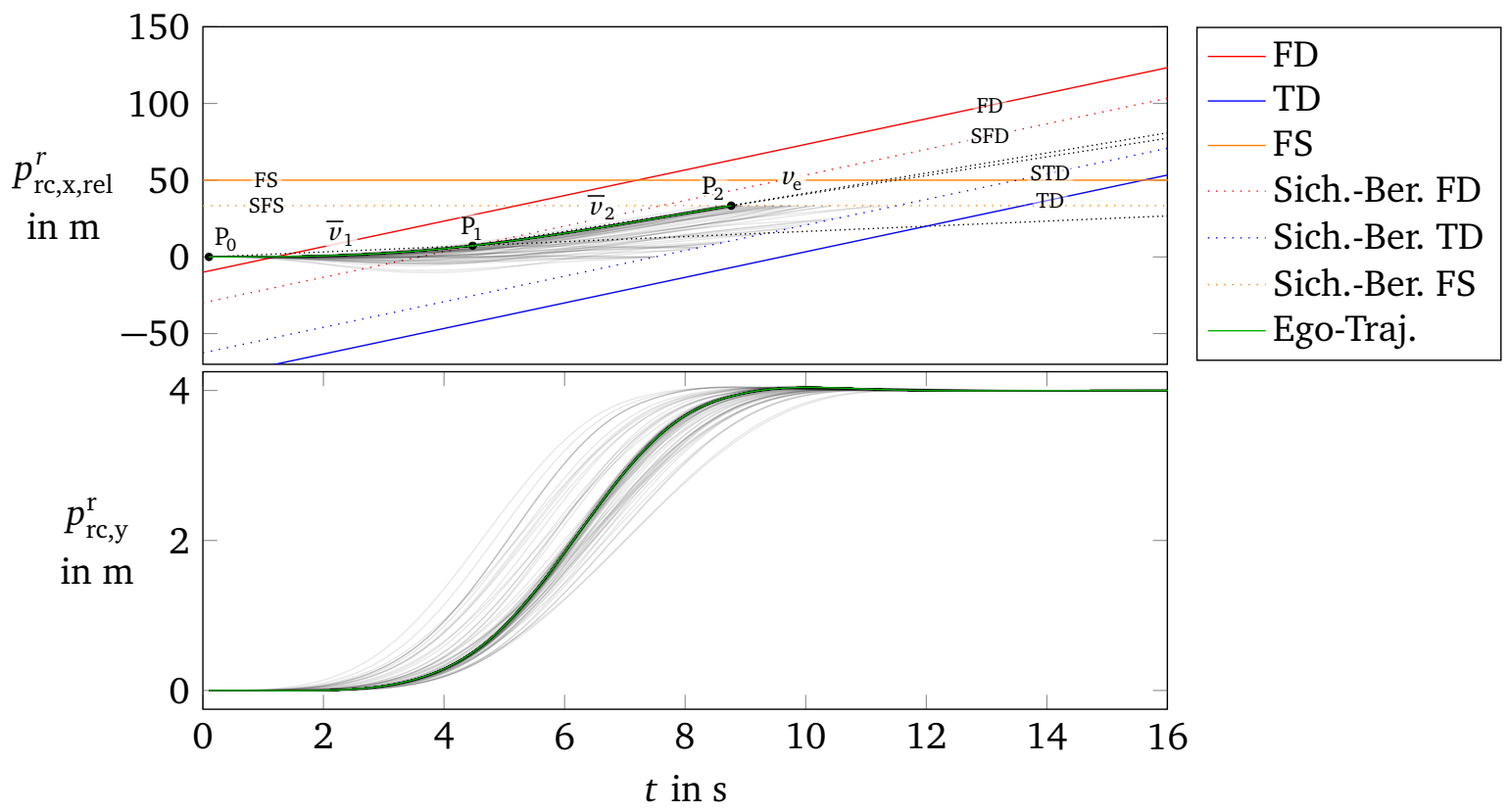

Abbildung 4.17: Ausgewertete Trajektorienkandidaten während eines Optimierungsdurchlaufes bzw. Planungsschrittes der Drei-Segment-Planung in Grau. Die beste und somit finale Trajektorie ist in Grün dargestellt. (Startwerte: $\bar{v}_{1}=\bar{v}_{2}=v_{\mathrm{e}}=$ $v_{\text {Ego }}$ ) 
Abbildung 4.17 zeigt die während der Optimierung ausgewerteten Trajektorienkandidaten. Als Startwerte für die Optimierungsvariablen wurde die aktuelle Geschwindigkeit des eigenen Fahrzeugs verwendet $\left(\bar{v}_{1}=\bar{v}_{2}=v_{\mathrm{e}}=v_{\mathrm{Ego}}\right)$.

Die finale Trajektorie wird anschließend an das Echtzeitsystem übertragen und aktiviert, sobald sie gültig ist. Solange dies noch nicht der Fall ist, wird weiter der alten Trajektorie gefolgt (siehe auch Abschnitt 2.7).

\subsection{Ergebnisse}

Die Trajektorienplanung wurde zunächst in der Simulation mit Hilfe der Software IPG CarMaker in diversen Szenarien getestet. Anschließend wurde eine ausgiebige Erprobung durch Testfahrten sowohl auf einem Testgelände als auch im realen Verkehr auf der Autobahn durchgeführt. Dabei konnte das System sichere und komfortable Fahrstreifenwechsel auch in dichtem Verkehr durchführen. Die Ergebnisse der Praxistests sind im Kapitel 6 ausführlicher dargestellt. Dort, sowie in Anhang C, werden auch die geplanten Trajektorien in unterschiedlichen Szenarien betrachtet und mit der Spline-Planung verglichen.

Durch die zyklische Neuplanung wird die Trajektorie im Verlauf des Fahrstreifenwechsels immer wieder an das Umfeld angepasst. Somit ergibt sich ein Sollgrößenverlauf, der ggf. von der ursprünglichen Trajektorie abweicht. Abbildung 4.18 zeigt den sich ergebenden Sollzustandsverlauf während eines realen Fahrstreifenwechsels auf dem Testgelände.

Aktuell wird als Startfahrstreifen der Fahrstreifen betrachtet, der neben dem Zielfahrstreifen noch vom Ego-Fahrzeug belegt wird. Diese Logik ist sehr einfach, führt jedoch dazu, dass sobald nur der Zielfahrstreifen belegt wird, die Trajektorien etwas länger ausfallen (Abbildung 4.18). Dies fiel bei den Fahrtests nicht negativ auf, sollte jedoch für einen Serieneinsatz durch einen erweiterten Ansatz ersetzt werden. Ab ca. $t=6 \mathrm{~s}$ ist der Fahrstreifenwechsel beendet und die Längsführung wird an das ACC übergeben. Es ist gut zu erkennen, dass der bis dahin ausgegebene Sollgrößenverlauf stetig ist, und das Fahrzeug den Fahrstreifen sicher wechselt. 


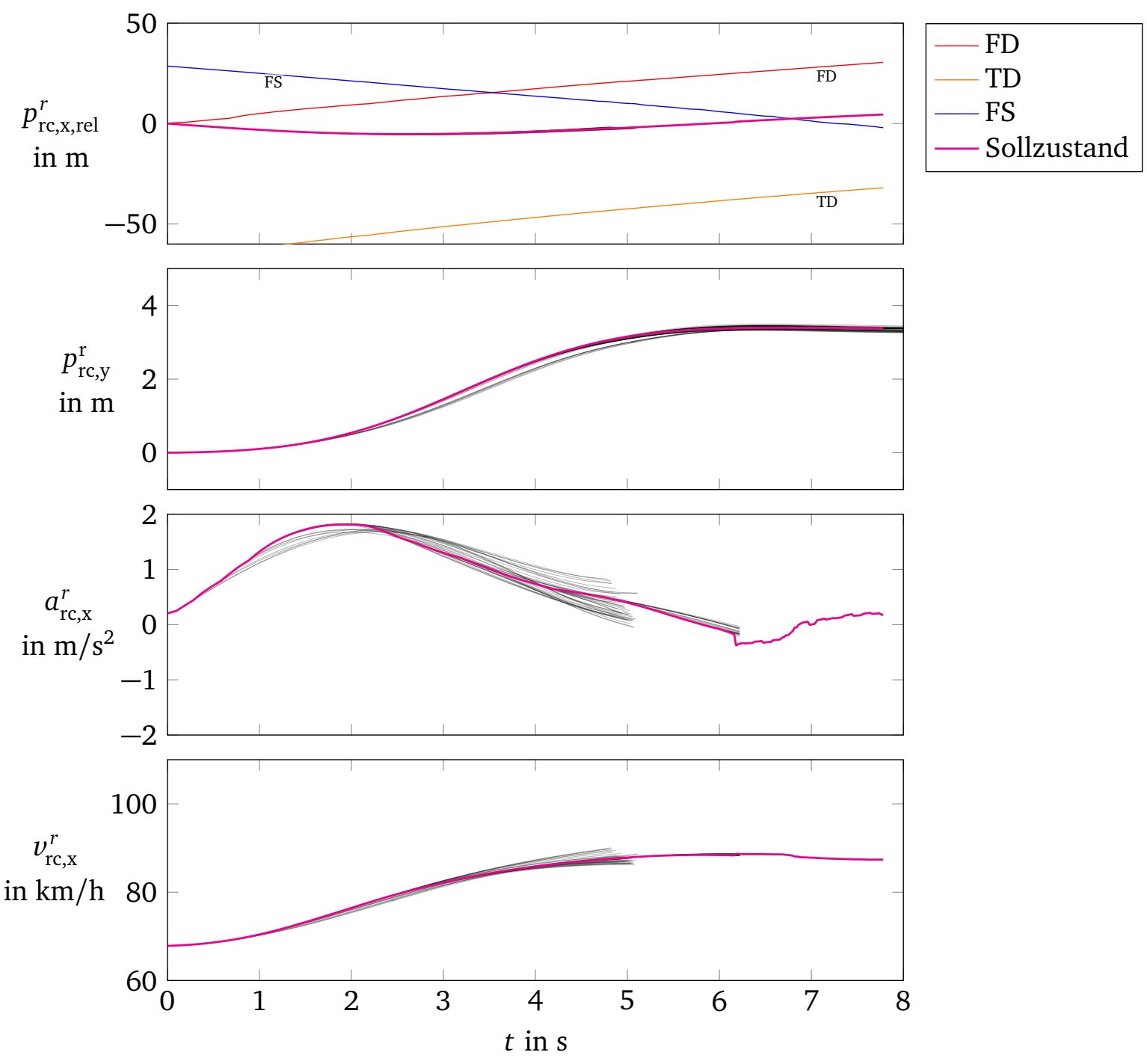

Abbildung 4.18: Verlauf des an die Regelung vorgegebenen Sollzustandes während eines realen Fahrstreifenwechsels nach links zwischen zwei schnellere Fahrzeuge. In Grau sind alle zwischenzeitlich geplanten Trajektorien dargestellt.

\subsection{Rechenzeit}

Zur Ermittlung der Auslastung eines möglichen Steuergerätes wurde für die Trajektorienplanung eine Abschätzung der notwendigen Operationen zur Berechnung der Gütefunktion vorgenommen. Hierbei wurde als Worst-Case-Szenario die Planung mit Drei-Segmenten herangezogen. Das Ergebnis für 10 Abtastpunkte pro Segment befindet sich in Tabelle 4.4. Unter Berücksichtigung der Tatsache, dass der Prozessor für die einzelnen Operationen unterschiedlich viele Instruktionen benötigt, ergeben sich, bei einer optimalen Implementierung auf dem angenommenen Steuergerät, 4240 Instruktionen. Je nach Effizienz der Implementierung kann es prinzipiell zu Abweichungen kommen. Bei einer Taktfrequenz von $1 \mathrm{GHz}$ werden für die Berechnung somit 4,24 $\mu$ s benötigt. 
Tabelle 4.4: Abschätzung über die notwendigen Operationen zur Berechnung der Gütefunktion der Drei-Segment-Planung für 10 Abtastpunkte pro Segment

\begin{tabular}{lccc} 
Typ & $\begin{array}{c}\text { Anzahl } \\
\text { Operationen }\end{array}$ & $\begin{array}{c}\text { Instruktionen } \\
\text { pro Operation }\end{array}$ & $\begin{array}{c}\text { Anzahl } \\
\text { Instruktionen }\end{array}$ \\
\hline Additionen & 1875 & 0,33 & 625 \\
Subtraktionen & 1673 & 0,33 & 557,67 \\
Multiplikationen & 3675 & 0,33 & 1225 \\
Divisionen & 158 & 6 & 948 \\
Logische Verknüpfungen (\&\&) & 29 & 0,33 & 9,67 \\
Vergleiche (<,>,...) & 1324 & 0,33 & 441,33 \\
Bedingte Zuweisungen & 1112 & 0,33 & 370,67 \\
Bedingte Sprünge (if) & 31 & 2 & 62 \\
\hline Summe der Instruktionen & & & $\underline{4}$
\end{tabular}

Im Rahmen der Optimierung muss die Gütefunktion mehrfach ausgewertet werden. Es wird davon ausgegangen, dass bei einem sich nicht sprunghaft ändernden Umfeld 100 Funktionsauswertungen ausreichend sein werden. Der Berechnungsaufwand für den Downhill-SimplexAlgorithmus wird vernachlässigt. Die Berechnung einer Trajektorie für eine Lücke benötigt daher $424 \mu \mathrm{s}$. Bei einer Neuplanungsrate von $5 \mathrm{~Hz}$ ergibt sich eine Auslastung des Steuergerätes von $0,21 \%$ pro berücksichtigter Lücke. Bei fünf Lücken liegt die Auslastung demnach bei $1,05 \%$. Diese fünf Lücken werden aber nur für das Anbieten eines Fahrstreifenwechsels berechnet. Während des Fahrstreifenwechsels wäre es denkbar, nur für zwei oder drei Lücken Trajektorien zu berechnen. Eine Zusammenfassung der Annahmen und Ergebnisse befindet sich in Tabelle 4.5.

Tabelle 4.5: Ergebnis der Rechenzeitbetrachtung der Drei-Segment-Planung

\begin{tabular}{lc} 
Bezeichnung & Wert \\
\hline Rechenzeit pro Trajektorienkandidat & $4,24 \mu \mathrm{s}$ \\
Trajektorienkandidaten pro Trajektorie & 100 \\
Rechenzeit pro Trajektorie & $424 \mu \mathrm{s}$ \\
Neuplanungen pro Sekunde & 5 \\
Auslastung für eine Lücke & $0,21 \%$ \\
Lücken für die Trajektorien berechnet werden & 5 \\
Auslastung für fünf Lücken & $1,05 \%$
\end{tabular}

Insgesamt ist also zu erwarten, dass die Planung für das angenommene Steuergerät geeignet ist. Es sei noch angemerkt, dass es relativ wahrscheinlich ist, dass für manche Lücken nur eine Trajektorie mit einem oder zwei Segmenten geplant werden muss. Deren Berechnung benötigt deutlich weniger Instruktionen. 


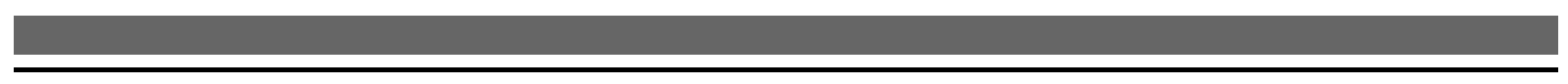




\section{Spline-Planung}

\subsection{Grundidee}

Nach dem Implementieren und Testen der Drei-Segment-Planung haben sich einige konzeptionelle Einschränkungen gezeigt:

1. Da die Trajektorie nur aus zwei Polynomen besteht, ist es schwer möglich, die maximal mögliche Beschleunigung des Fahrzeugs über eine gewisse Zeit aufrecht zu halten.

2. Fahrzeuge, die einen Fahrstreifen nur teilweise belegen oder den Fahrstreifen wechseln, können bei der Planung nicht entsprechend berücksichtigt werden.

3. Die Berücksichtigung der auftretenden Fallunterscheidungen zur Bestimmung der Segmentanzahl ist teilweise komplex und in einigen Fällen ist eine Planung zurzeit nicht möglich (Anhang A.6).

Um diese Einschränkungen zu vermeiden, wird ein Spline-basierter Ansatz untersucht. Auch hier ist die Trajektorie Ergebnis einer Optimierung. Die Trajektorie hat eine feste zeitliche Länge und deutlich mehr Freiheitsgrade. Die Gütefunktion ist auf dem gesamten Suchraum definiert und stetig differenzierbar. Der Gradient der Gütefunktion ist analytisch berechenbar, so dass die Rechenzeit akzeptabel bleibt. Eine Rückübergabe an das ACC findet nicht statt, da die Trajektorienplanung auch für die Folgefahrt verwendet wird.

\subsection{Generierung der Trajektorie}
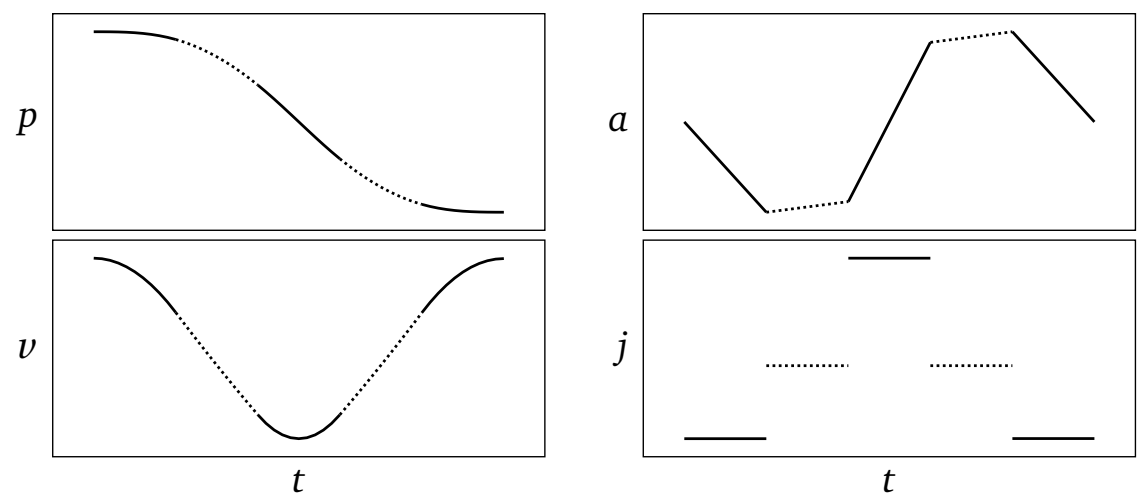

Abbildung 5.1: Spline-Beispieltrajektorie mit 5 Segmenten für die Querrichtung

Die Trajektorie besteht aus vielen Segmenten gleicher Dauer. In dieser Arbeit werden dazu $n=$ 15 Segmente à $T=1 \mathrm{~s}$ verwendet. Für jedes Segment und jede Richtung (Längs und Quer) wird 
ein Polynom 3. Grades verwendet. Da ein stetiger Verlauf der Größen Position, Geschwindigkeit und Beschleunigung gefordert wird, sind von den 4 Polynomkoeffizienten 3 durch die jeweiligen Anfangsbedingungen festgelegt. Für jedes Segment und jede Richtung bleibt daher ein freier Parameter. Im Rahmen dieses Projektes wurde hierfür der Ruck verwendet. Die Verwendung der Endposition ist ebenfalls denkbar und gegebenenfalls numerisch vorteilhafter (Anhang B.2). Abbildung 5.1 zeigt eine Beispieltrajektorie mit 5 Segmenten.

Ein solcher Spline mit Polynomen 3. Grades stellt auch den exakten Verlauf eines Dreifachintegrators bei einem abschnittsweise konstanten Eingang dar (Abbildung 5.2).

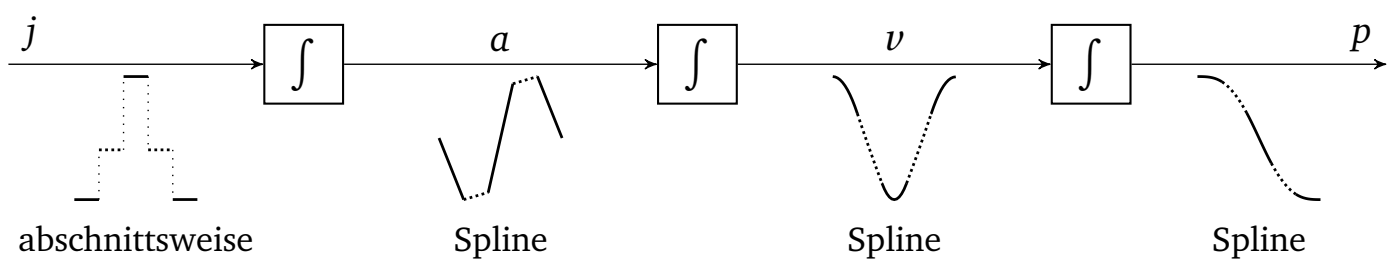
konstant
1. Grades
2. Grades
3. Grades

Abbildung 5.2: Analogie zwischen Spline-Trajektorie und Dreifachintegrator

Die Trajektorienlänge von $15 \mathrm{~s}$ ergibt sich aus der Tatsache, dass der komplette Fahrstreifenwechsel inklusive der Anfahrt geplant werden muss, bevor er begonnen werden kann. Bei der Drei-Segment-Planung hat sich gezeigt, dass eine Dauer von ungefähr $8 \mathrm{~s}$ für die Querbewegung als komfortabel empfunden wird. Somit bleibt für die Anfahrt zum eigentlichen Fahrstreifenwechsel noch eine Zeit von $7 \mathrm{~s}$. Zur Reduktion des Berechnungsaufwandes ist es jedoch prinzipiell auch möglich, einen kürzeren Planungshorizont zu verwenden. Die Segmentlänge von $1 \mathrm{~s}$ wurde gewählt um auch noch schnelle Fahrstreifenwechsel oder Abbruchszenarien gut abbilden zu können.

Im Folgenden wird ein kurzer Überblick über die Generierung der Trajektorie gegeben. Die ausführliche, mathematische Beschreibung befindet sich in Anhang B.1.

Verwendete Symbole:

Symbol Beschreibung

$\begin{array}{ll}T & \text { Dauer eines Segmentes } \\ n & \text { Anzahl Segmente } \\ \underline{\mathbf{p}}_{i} & \text { Polynomkoeffizienten für das Segment } i \\ \underline{\mathbf{x}}_{0} & \text { Anfangszustand }\left[p_{0}, v_{0}, a_{0}\right]^{\mathrm{T}} \text { zum Zeitpunkt } t=0\end{array}$


Die Spline-Trajektorie $\underline{\mathbf{f}}(t)$ setzt sich aus $n$ Teilfunktionen $\underline{\mathbf{f}}_{i}(\tau)$ zusammen. Jede der Teilfunktionen läuft von $\tau=0$ bis $\tau=T$ :

$$
\left[\begin{array}{l}
p(t) \\
v(t) \\
a(t) \\
j(t)
\end{array}\right]=\underline{\mathbf{f}}(t)= \begin{cases}\underline{\mathbf{f}}_{1}(t) & 0 \leq t \leq T \\
\underline{\mathbf{f}}_{2}(t-T) & T \leq t \leq 2 T \\
\vdots & \vdots \\
\underline{\mathbf{f}}_{n}(t-(n-1) T) & (n-1) T \leq t \leq n T\end{cases}
$$

Mit Hilfe der Basisfunktion $\underline{\mathbf{F}}_{\mathrm{b}}^{\mathrm{T}}(\tau)$ und den Polynomkoeffizienten $\underline{\mathbf{p}}_{i}$ kann der Verlauf der Zustandsgrößen $p, v, a$ und $j$ in einem Segment über der Zeit dargestellt werden:

$$
\underline{\mathbf{f}}_{i}(\tau)=\left[\begin{array}{c}
p_{i}(\tau) \\
v_{i}(\tau) \\
a_{i}(\tau) \\
j_{i}(\tau)
\end{array}\right]=\underbrace{\left[\begin{array}{cccc}
1 & \tau & \tau^{2} & \tau^{3} \\
0 & 1 & 2 \tau & 3 \tau^{2} \\
0 & 0 & 2 & 6 \tau \\
0 & 0 & 0 & 6
\end{array}\right]}_{\underline{\mathrm{F}}_{\mathrm{b}}^{\mathrm{T}}(\tau)} \underline{\mathbf{p}}_{i} \quad \underline{\mathbf{p}}_{i} \in \mathbb{R}^{4 \times 1}
$$

Da ein stetiger Übergang der ersten 3 Ableitungen gefordert wird, reduziert sich die Anzahl der Freiheitsgrade in den Polynomkoeffizienten $\mathbf{p}_{i}$. Im Anhang B.1 wird gezeigt, dass sich die Polynomkoeffizienten in Abhängigkeit des Anfangzustands $\underline{\underline{x}}_{0}=\left[\begin{array}{lll}p_{0} & v_{0} & a_{0}\end{array}\right]^{\mathrm{T}}$ und den Rucken $\underline{\mathbf{j}}$ in den einzelnen Segmenten wie folgt darstellen lassen:

$$
\underline{\mathbf{p}}_{i}=\underline{\mathbf{D}}_{i} \cdot \underline{\mathbf{x}}_{0}+\underline{\mathbf{E}}_{i} \cdot \underline{\mathbf{j}} \quad \underline{\mathbf{D}}_{i} \in \mathbb{R}^{4 \times 3} \quad \underline{\mathbf{E}}_{i} \in \mathbb{R}^{4 \times n} \quad \underline{\mathbf{j}} \in \mathbb{R}^{n \times 1}
$$

Die Matrizen $\underline{\mathbf{D}}_{i}$ und $\underline{\mathbf{E}}_{i}$ sind hierbei für jedes Segment verschieden, jedoch konstant und lassen sich offline bestimmen. Durch diese Form wird die Trajektorie vollständig durch den Anfangszustand $\underline{\mathbf{x}}_{0}$ sowie den Parametervektor $\underline{\mathbf{j}}$ beschrieben und die Stetigkeitsbedingungen sind automatisch erfüllt. Dieses Vorgehen wird sowohl auf die Längs- als auch die Querrichtung angewendet, wobei hierfür natürlich unterschliedliche $\underline{\mathbf{x}}_{0}$ und $\mathbf{j}$ verwendet werden.

Auch die Polynomkoeffizienten $\underline{\mathbf{p}}_{i}$ müssen nicht online bestimmt werden, sondern werden weiter in die Formeln des nachfolgend beschriebenen analytischen Gütemaßes und der Abtastung (Abschnitt 5.3) eingesetzt. Auf diese Weise können sehr viele Berechnungen offline erfolgen. 


\section{Analytisches Gütemaß}

Symbol Beschreibung

\begin{tabular}{ll}
\hline$\underline{\mathbf{x}}_{0}$ & Anfangszustand \\
$\underline{\mathbf{x}}_{\mathrm{e}}$ & Soll-Endzustand \\
$\underline{\mathbf{W}}_{\mathrm{A}}$ & Diagonale Gewichtungsmatrix (positiv semidefinit) \\
$\underline{\mathbf{W}}_{\mathrm{B}}$ & Diagonale Endpunktgewichtungsmatrix (positiv semidefinit)
\end{tabular}

Häufig besitzt ein Gütefunktional die Form:

$$
J_{\mathrm{A}}=\int_{0}^{n T}\left[\underline{\mathbf{f}}^{\mathrm{T}}(t) \underline{\mathbf{W}}_{\mathrm{A}} \underline{\mathbf{f}}(t)\right] \mathrm{d} t
$$

Dies gilt insbesondere für die Größen, die den Fahrkomfort beschreiben. In Anhang B.1 wird gezeigt, dass es für diese Planung möglich ist, das Gütemaß in folgende Form zu bringen:

$$
J_{\mathrm{A}}=\underline{\mathbf{j}} \underline{\mathbf{F}}^{\mathrm{T}} \underline{\mathbf{j}}+\underline{\mathbf{x}}_{0}^{\mathrm{T}} \underline{\mathbf{G}}_{\mathrm{A}} \underline{\mathbf{j}}+\underline{\mathbf{x}}_{0}^{\mathrm{T}} \underline{\mathbf{H}}_{\mathrm{A}} \underline{\mathbf{x}}_{0}
$$

Auch eine Endpunktgewichtung in der Form

$$
J_{\mathrm{B}}=\left(\underline{\mathbf{x}}_{n}-\underline{\mathbf{x}}_{\mathrm{e}}\right)^{\mathrm{T}} \underline{\mathbf{W}}_{\mathrm{B}}\left(\underline{\mathbf{x}}_{n}-\underline{\mathbf{x}}_{\mathrm{e}}\right)
$$

lässt sich zu

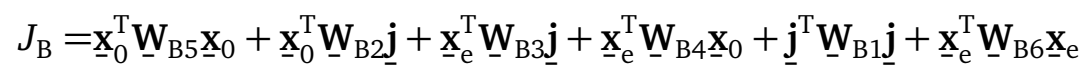

umformen. Hierbei können die Matrizen $\underline{\mathbf{F}}_{\mathrm{A}}, \underline{\mathbf{G}}_{\mathrm{A}}$ und $\underline{\mathbf{H}}_{\mathrm{A}}$ sowie $\underline{\mathbf{W}}_{\mathrm{B} 1}$ bis $\underline{\mathbf{W}}_{\mathrm{B} 6}$ offline berechnet werden. Details dazu sind in Anhang B.1.2 beschrieben. Die Endpunktbewertung wird in Querrichtung verwendet, um zu erreichen, dass die Trajektorie möglichst in der Mitte des Zielfahrstreifens sowie ohne Quergeschwindigkeit und -beschleunigung endet.

\section{Längs- und Querrichtung}

Es wird je ein Spline in Längs- und einer in Querrichtung verwendet. Hierbei werden für den Komfort folgende normierte Gewichtungen verwendet:

$$
\underline{\mathbf{W}}_{\text {Lon,A }}=\left[\begin{array}{cccc}
0 & 0 & 0 & 0 \\
0 & 0 & 0 & 0 \\
0 & 0 & 0,1 & 0 \\
0 & 0 & 0 & 0,02
\end{array}\right] \quad \underline{\mathbf{W}}_{\text {Lat }, \mathrm{A}}=\left[\begin{array}{cccc}
0 & 0 & 0 & 0 \\
0 & 3 & 0 & 0 \\
0 & 0 & 0,5 & 0 \\
0 & 0 & 0 & 1
\end{array}\right]
$$

Und für die Endpunktbewertung:

$$
\underline{\mathbf{W}}_{\text {Lon, } \mathrm{B}}=\left[\begin{array}{ccc}
0 & 0 & 0 \\
0 & 0 & 0 \\
0 & 0 & 0
\end{array}\right] \quad \mathbf{W}_{\mathrm{Lat}, \mathrm{B}}=\left[\begin{array}{ccc}
1 & 0 & 0 \\
0 & 1 & 0 \\
0 & 0 & 1
\end{array}\right] \cdot 10^{2}
$$

Die Parameter wurden experimentell bestimmt. Durch Variation ist es möglich, die Trajektorien an unterschiedliche Kundenwünsche und Fahrzeuge anzupassen. Damit entstehen abschließend für den Komfort die Gütemaße $J_{\text {Lon }}$ und $J_{\text {Lat }}$ sowie für die Quer-Endpunktbewertung $J_{\text {Lat,End }}$. 


\subsection{Abtastung der Trajektorie}

Verwendete Symbole:

Symbol Beschreibung

$m \quad$ Anzahl Abtastpunkte pro Segment

$n \quad$ Anzahl Segmente

Neben den oben dargestellten Formen (5.4) und (5.6) gibt es auch noch weitere Terme im Gütemaß, die sich nicht so einfach analytisch darstellen lassen. Die Berechnung dieser Gütemaße basiert hierbei auf den Abtastpunkten des Splines.



Abbildung 5.3: Die einzelnen Segmente einer Spline-Trajektorie zusammen mit den Abtastpunkten

Für jedes Segment werden in dieser Arbeit $m=2$ Abtastpunkte verwendet. Der Startpunkt entfällt, da er nicht verändert werden kann. Damit ergeben sich $m \cdot n=30$ Abtastpunkte (Abbildung 5.3).

In Anhang B.1.3 wird gezeigt, dass es möglich ist, den Abtastvorgang als Multiplikation mit konstanten Matrizen darzustellen. Für die Position ergibt sich beispielsweise folgende Gleichung:

$$
\underline{\mathbf{s}}_{\mathrm{p}}=\underline{\mathbf{L}}_{\mathrm{p}} \underline{\mathbf{x}}_{0}+\underline{\mathbf{M}}_{\mathrm{p}} \mathbf{j} \quad \underline{\mathbf{L}}_{\mathrm{p}} \in \mathbb{R}^{m \cdot n \times 3} \quad \underline{\mathbf{M}}_{\mathrm{p}} \in \mathbb{R}^{m \cdot n \times n}
$$

Aufgrund dieser Tatsache ist es möglich, eine Gütefunktion $J_{\mathrm{p}}\left(\underline{\mathbf{s}}_{\mathrm{p}}\right)$ in Abhängigkeit der Position $\underline{\mathbf{s}}_{\mathrm{p}}$ an den Abtastpunkten zu formulieren und anschließend den Gradienten umzurechnen:

$$
\frac{\mathrm{d} J_{\mathrm{p}}}{\mathrm{d} \underline{\mathbf{j}}}=\frac{\mathrm{d} J_{\mathrm{p}}}{\mathrm{d} \underline{\mathbf{s}}_{\mathrm{p}}} \cdot \underline{\mathbf{M}}_{\mathrm{p}}
$$

Die einzelnen Gradienten der Gütefunktionen können so auf Ebene der Abtastpunkte summiert werden und anschließend auf $\mathbf{j}$ umgerechnet werden. Dies spart Rechenzeit gegenüber der direkten Berechnung jedes einzelnen Gradientens bzgl. $\underline{\mathbf{j}}$.

Die abgetasteten Größen befinden sich in Tabelle 5.1. 
Tabelle 5.1: Abgetastete Größen bei der Spline-Planung

\begin{tabular}{lcc} 
& Längs & Quer \\
\hline Position & $p_{\text {Lon }}$ & $p_{\text {Lat }}$ \\
Geschwindigkeit & $a_{\text {Lon }}$ & $a_{\text {Lat }}$ \\
Beschleunigung & $v_{\text {Lon }}$ & $v_{\text {Lat }}$ \\
Ruck & $j_{\text {Lon }}$ & $j_{\text {Lat }}$
\end{tabular}

\section{4 Überblick}

Eine Trajektorie setzt sich aus einem Spline in Quer- und einem in Längsrichtung zusammen. Die Anfangsbedingungen der Splines werden so gewählt, dass eine stetige Trajektorie entsteht. Als Startwerte für die Optimierungsvariablen werden die Werte der vorherigen Planung verwendet. Basierend darauf wird die Trajektorie generiert, der Komfort in Form der integralen Gütemaße (5.4) bestimmt sowie der Endpunkt der Trajektorie gemäß (5.6) bewertet. Anschließend wird die Trajektorie abgetastet und basierend darauf werden weitere Bewertungen durchgeführt. Diese Bewertungen geben Gradienten bzgl. der Abtastpunkte aus, die sich auf die eigentlichen Optimierungsvariablen $\mathbf{j}$ umrechnen lassen. Die einzelnen Güteterme sowie deren Gradienten werden addiert und dem Optimierungsalgorithmus für nichtlineare Probleme zur Verfügung gestellt. Aktuell wird dafür der L-BFGS-Algorithmus verwendet (Abschnitt 5.8). Dieser nutzt den Gradienten, um iterativ neue Kandidaten für die Optimierungsvariablen zu bestimmen. Wenn das Minimum gefunden wurde, oder eine maximale Anzahl von Funktionsauswertungen erreicht wurde, bricht der Algorithmus ab (Abbildung 5.4).

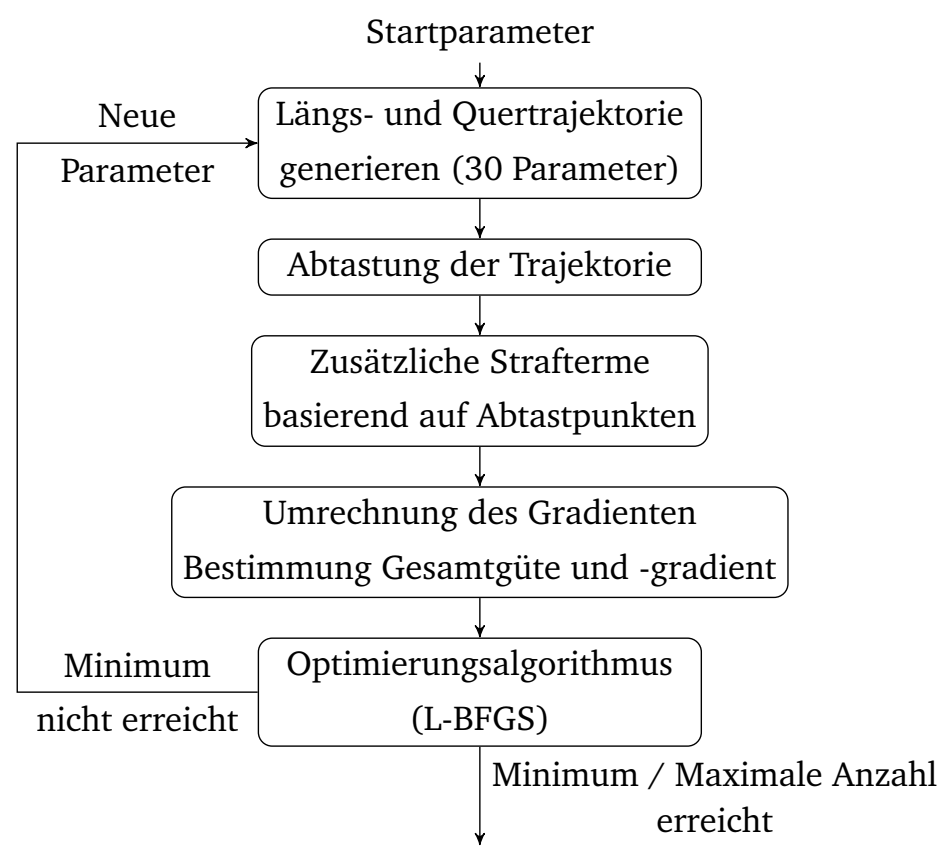

Abbildung 5.4: Übersicht über den Ablauf der Spline-Trajektorienplanung 


\subsection{Umfeldprädiktion}

Um zu vermeiden, dass die geplante Trajektorie zu einer Kollision mit den Umfeldfahrzeugen führt, muss das zukünftige Verhalten der Umfeldfahrzeuge prädiziert werden. Aufgrund von Einschränkungen in der Umfelderfassung findet die Prädiktion in dieser Arbeit ausschließlich in Längsrichtung statt. In Querrichtung wird davon ausgegangen, dass die Umfeldfahrzeuge den Fahrstreifen nicht wechseln.

Eigentlich müsste die Prädiktion der Umfeldobjekte die Bewegung des eigenen Fahrzeugs berücksichtigen und somit für jeden Trajektorienkandidaten neu durchgeführt werden. Dies ist möglich, allerdings gestaltet sich die Berechnung des Gradienten dann relativ aufwendig. Aus Komplexitätsgründen und zur Reduktion der Rechenzeit wurde die Bewegung des eigenen Fahrzeugs daher nicht berücksichtigt.

Es ist Aufgabe des Fahrers des rückwärtigen Fahrzeugs, den Sicherheitsabstand zum EgoFahrzeug einzuhalten. Um nicht auf ein eventuelles Drängeln zu reagieren, wird daher, wie bei der Drei-Segment-Planung, das rückwärtige Fahrzeug auf dem aktuell befahrenen Fahrstreifen ausgeblendet. Hierbei zählt der Fahrstreifen, auf dem sich die Mitte der Hinterachse befindet. Denkbar wäre jedoch auch ein Ausblenden erst nachdem der Fahrstreifen eine gewisse Zeit lang befahren wurde oder alternativ eine gesonderte Prädiktion des rückwärtigen Verkehrs.

Es hat sich gezeigt, dass die $15 \mathrm{~s}$ langen Trajektorien hohe Anforderungen an die Prädiktion des Umfeldes stellen. Für die Prädiktion wurden 3 unterschiedliche Ansätze untersucht.

\subsubsection{Prädiktion mit konstanter Geschwindigkeit}
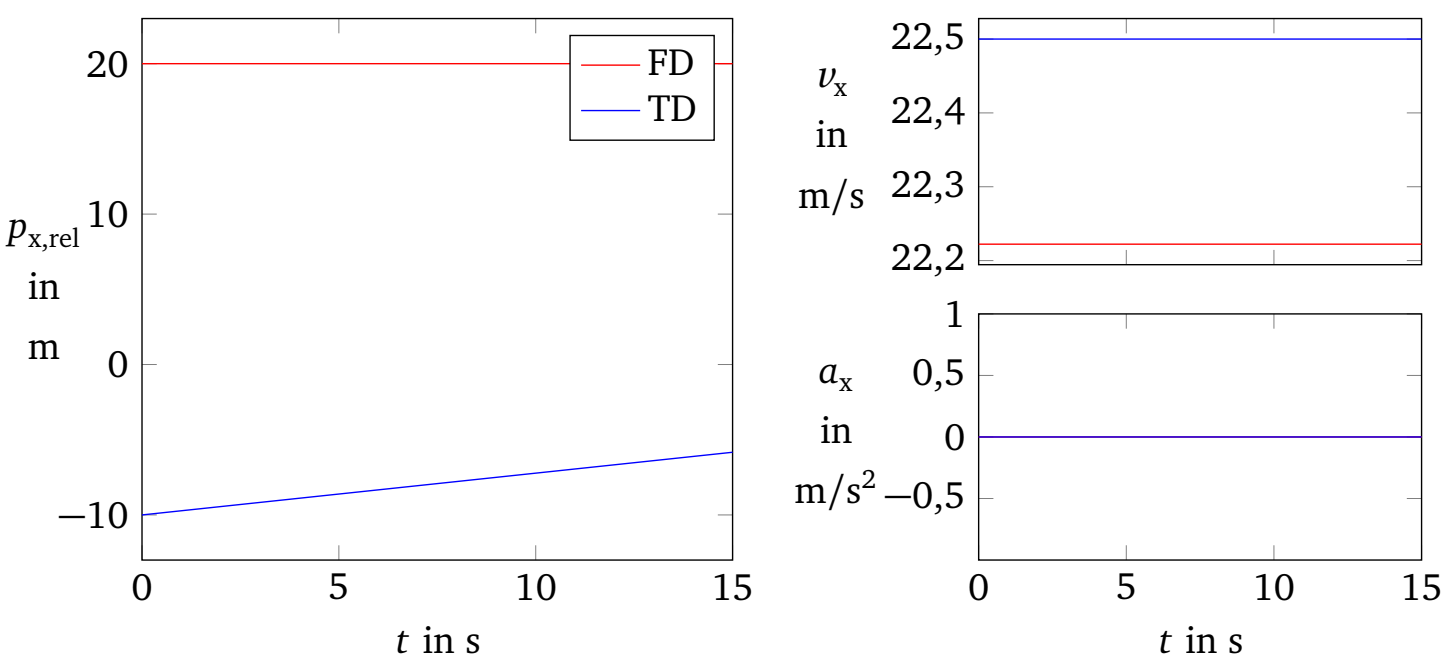

Abbildung 5.5: Beispiel für die Prädiktion der Umfeldobjekte mit konstanter Geschwindigkeit

Bei dieser Variante werden die Umfeldfahrzeuge, analog zur Drei-Segment-Planung, mit ihrer aktuellen Längsgeschwindigkeit in die Zukunft prädiziert (Abbildung 5.5). Bei ersten Tests 
hat sich jedoch gezeigt, dass diese Annahme zu optimistisch ist. In Situationen, in denen das vorausfahrende Fahrzeug verzögert, hat dies zur Folge, dass das Ego-Fahrzeug sehr dicht auffährt.

\subsubsection{Prädiktion unter Ausnutzung der Beschleunigung}

Von der Umfelderfassung wird ein Schätzwert für die Beschleunigung der Objekte ausgegeben. Wird diese Schätzung verwendet, lässt sich die Prädiktion verbessern. Eine Prädiktion mit konstanter Beschleunigung ist jedoch wenig zielführend. Denn selbst bei einer kleinen Verzögerung von beispielsweise $1 \mathrm{~m} / \mathrm{s}^{2}$ entsteht am Ende des Prädiktionshorizonts eine Geschwindigkeitsdifferenz von $15 \mathrm{~m} / \mathrm{s}=54 \mathrm{~km} / \mathrm{h}$. Dies ist wenig realistisch. Aus diesem Grund wird die zur Prädiktion verwendete Beschleunigung langsam ausklingen gelassen. Hierbei wird in dieser Arbeit eine Zeitkonstante von $5 \mathrm{~s}$ verwendet (Abbildung 5.6). Eine Begrenzung sorgt dafür, dass das prädizierte Fahrzeug keine negativen Geschwindigkeiten annimmt.
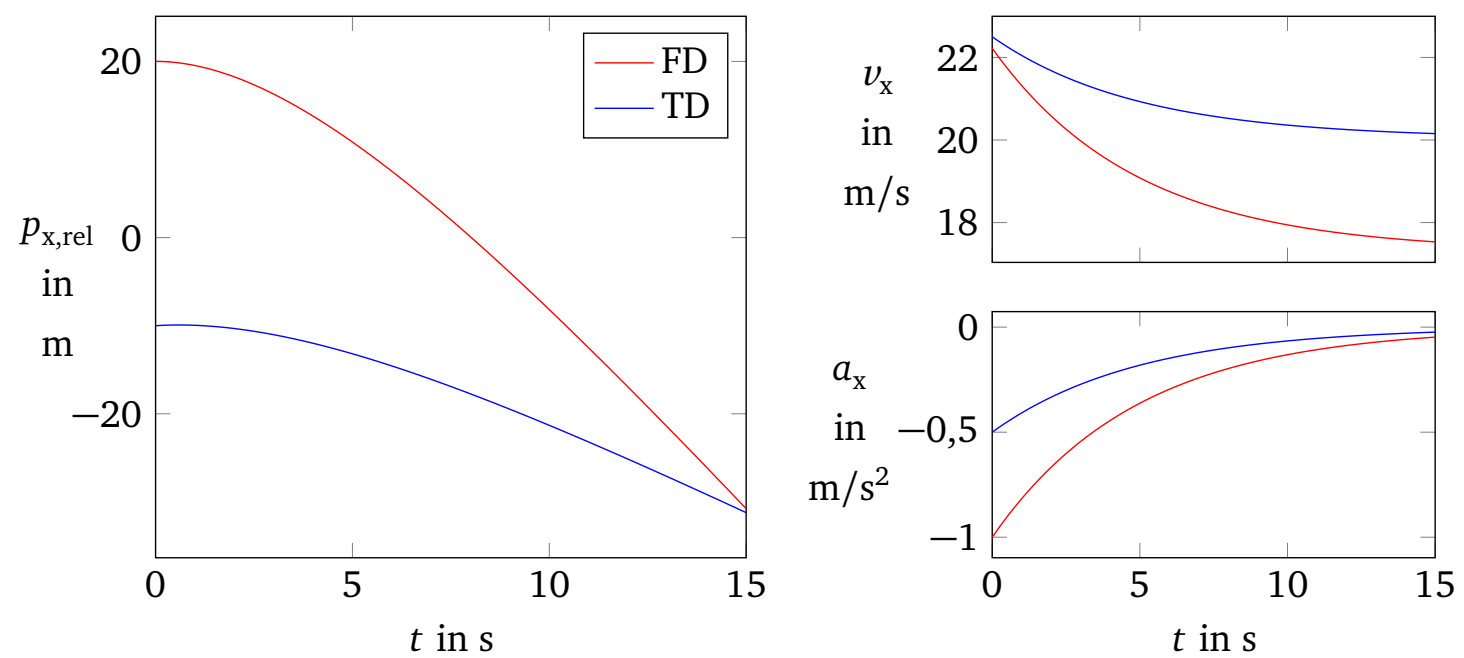

Abbildung 5.6: Beispiel für die Prädiktion der Umfeldobjekte unter Ausnutzung der Beschleunigung

Nachteilig bei dieser Art der Prädiktion ist zum Beispiel der Fall, wenn das hintere Fahrzeug auf dem Zielfahrstreifen schwächer verzögert als das vordere. Es kommt zu dem Effekt, dass die Lücke am Ende der Trajektorie regelrecht verschwunden ist. Es gibt zwischen den Fahrzeugen FD und TD scheinbar keinen Platz mehr für das eigene Fahrzeug. Der Güteterm zur Kollisionsvermeidung wäre in diesem Fall sehr groß, und der Fahrstreifenwechsel würde abgebrochen. In der Praxis hält der Fahrer des rückwärtigen Fahrzeugs jedoch einen ausreichenden Abstand zum vorausfahrenden Fahrzeug, sodass ein Wechsel in solche Lücken meistens möglich ist. Da dieser Fall in der Praxis sehr häufig auftaucht, wurde eine verbesserte Variante entwickelt, die im Folgenden beschrieben wird. 
Um eine realistischere Prädiktion des rückwärtigen Fahrers zu erhalten, wird für ihn ein einfaches Fahrermodell angenommen. Hierzu kommt wieder das Intelligent-Driver-Model (IDM) zum Einsatz [Treiber, 2010, S.162f.]. Die maximale Verzögerung, mit der der rückwärtige Verkehr prädiziert wird, beträgt in dieser Arbeit $0,7 \mathrm{~m} / \mathrm{s}^{2}$ (Abbildung 5.7). Durch diese Begrenzung reagiert man in Situationen, in denen das hintere Fahrzeug aufgrund des vorderen Fahrzeugs stärker bremsen muss, eher konservativ und bricht den Fahrstreifenwechsel ab.
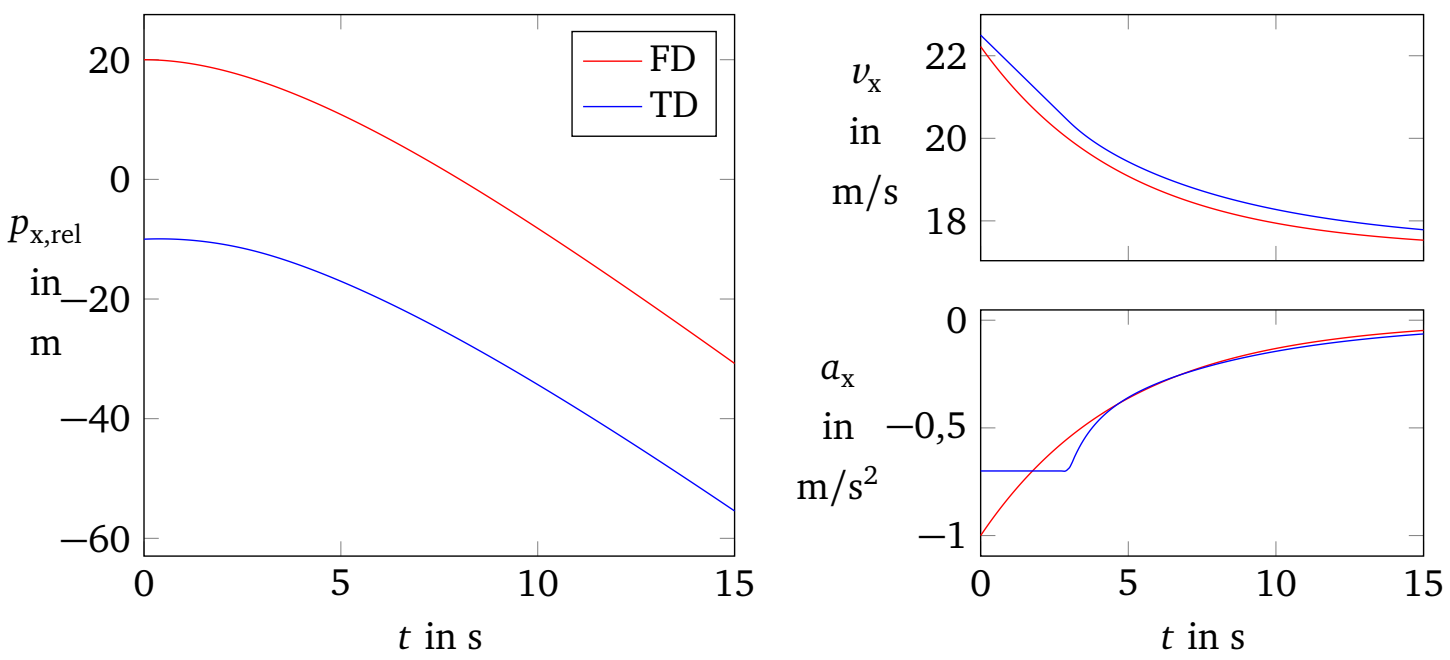

Abbildung 5.7: Beispiel für die Prädiktion der Umfeldobjekte unter Ausnutzung der Beschleunigung und eines Fahrermodells

Diese Art der Prädiktion hat sich in den Fahrversuchen als geeignet herausgestellt und wurde in der finalen Version verwendet.

\subsection{Straffunktionen}

Basierend auf den Abtastpunkten der Trajektorie und des Umfeldes werden verschiedene einzelne Strafterme berechnet, die dann gewichtet und anschließend aufsummiert werden. Die Optimierung berücksichtigt hierbei keine harten Beschränkungen an die Trajektorie. Beschränkungen sind mit in die Gütefunktion integriert. Dies ist zum einen günstig für die benötigte Rechenzeit, zum anderen hat es den Vorteil, dass immer eine Trajektorie planbar ist.

Aufgrund von Messfehlern oder eines sich verändernden Umfeldes kann es ansonsten dazu kommen, dass keine Trajektorie planbar ist. Es hat sich gezeigt, dass es in diesen Situationen besser ist, das Fahrzeug weiter zu führen und den Fahrer zur Übernahme aufzufordern, anstatt die Fahrzeugführung sofort zu beenden. Die ausgegebene Trajektorie ist in diesem Fall immer noch die bestmögliche. Für einen Serieneinsatz wäre es sinnvoll, die endgültige Trajektorie zusätzlich gegen harte Beschränkungen zu testen, bevor sie auf das Fahrzeug gegeben wird. 
Folgende Punkte werden durch die Straffunktionen abgedeckt:

- Vermeidung von Kollisionen

- Einhaltung der Sicherheitsabstände

- Berücksichtigung der Fahrstreifen

- Begrenzte Fahrzeugdynamik

- Endsituation

Alle diese Straffunktionen müssen stetig differenzierbar sein und berechnen den Gradienten bzgl. der Abtastpunkte. Dieser wird später mittels Gleichung (5.9) auf die eigentlichen Optimierungsvariablen $\underline{\mathbf{j}}$ umgerechnet. Viele der Terme definieren ein Potential $\phi_{\text {.... }}$ über das in der Form

$$
J_{\ldots}=\int_{0}^{n T} \phi_{\ldots}\left(\underline{\mathbf{x}}_{\mathrm{Lon}}(t), \underline{\mathrm{x}}_{\mathrm{Lat}}(t)\right) \mathrm{d} t
$$

integriert wird. Die einzelnen Terme werden abschließend gewichtet und addiert. Prinzipiell wäre dies auch auf Ebene des Potentials möglich, würde jedoch mehr Rechenzeit benötigen als eine Gewichtung der Endterme. Im Folgenden werden die einzelnen Komponenten des Gütemaßes näher beschrieben. Wieder wurde Wert gelegt auf eine einfache Darstellung zum Zwecke der Rechenzeitoptimierung sowie einer guten Parametrierbarkeit.

\subsection{1 $J_{\mathrm{AC}}-$ Kollisionsvermeidung}

Eine wesentliche Aufgabe der Trajektorienplanung ist die Vermeidung von Kollisionen. Um dies zu erreichen, darf weder eine Kollision mit den vier betrachteten Umfeldfahrzeugen auftreten, noch dürfen andere, unbeteiligte Fahrstreifen befahren werden. Das verwendete Potential ist ähnlich zu $\phi_{\text {SafetyArea }}$ der Drei-Segment-Planung. Im Gegensatz dazu ist es aber stetig differenzierbar und verwendet kleinere Bereiche.

\section{Umfeldfahrzeuge}

Betrachtet wird zunächst beispielhaft das Umfeldfahrzeug FD. Für jedes Umfeldfahrzeug wird ein, von der Geschwindigkeit des Umfeldobjektes abhängiger, nicht zu befahrender Bereich bestimmt. Wie auch bei der Drei-Segment-Planung wird der Fahrstreifen, der vom Umfeldfahrzeug befahren wird, über seine gesamte Breite als belegt betrachtet. Der Bereich orientiert sich daher in Querrichtung an den Fahrstreifenmarkierungen. Die Bestrafung beginnt ab einem Abstand von 0,5 m zwischen der Kante des eigenen Fahrzeugs und der Fahrstreifenmarkierung (Abbildung 5.8). Für die Ausdehnung in Längsrichtung gilt:

$$
\Delta x_{\mathrm{AC}, \mathrm{FD}}=\Delta T_{\mathrm{AC}, \mathrm{Front}} \cdot v_{\mathrm{FD}}+\Delta x_{0, \mathrm{AC}, \mathrm{Front}}
$$


- $\Delta T_{\mathrm{AC}, \text { Front }}$ : Zeitlücke (geschwindigkeitsabhängiger Anteil)

- $\Delta x_{0, \mathrm{AC}, \text { Front }}:$ konstanter Term

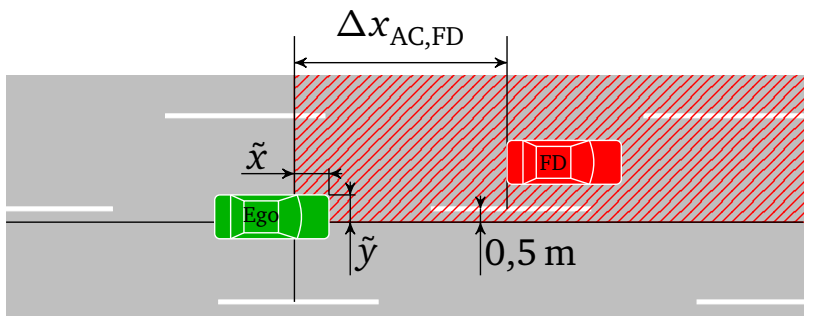

Abbildung 5.8: Bereich zur Vermeidung von Kollisionen mit dem Umfeldfahrzeug FD

Ein Eindringen in diesen Bereich wird quadratisch bestraft. Abbildung 5.8 stellt die dabei verwendeten Größen $\tilde{x}$ und $\tilde{y}$ dar, Abbildung 5.9 die sich ergebende Potentialfunktion $\phi_{\mathrm{AC}, \mathrm{FD}}$ :

$$
\phi_{\mathrm{AC}, \mathrm{FD}}(\tilde{x}, \tilde{y})= \begin{cases}\tilde{x}^{2} \cdot \tilde{y}^{2} & \tilde{x}>0 \wedge \tilde{y}>0 \\ 0 & \tilde{x} \leq 0 \vee \tilde{y} \leq 0\end{cases}
$$

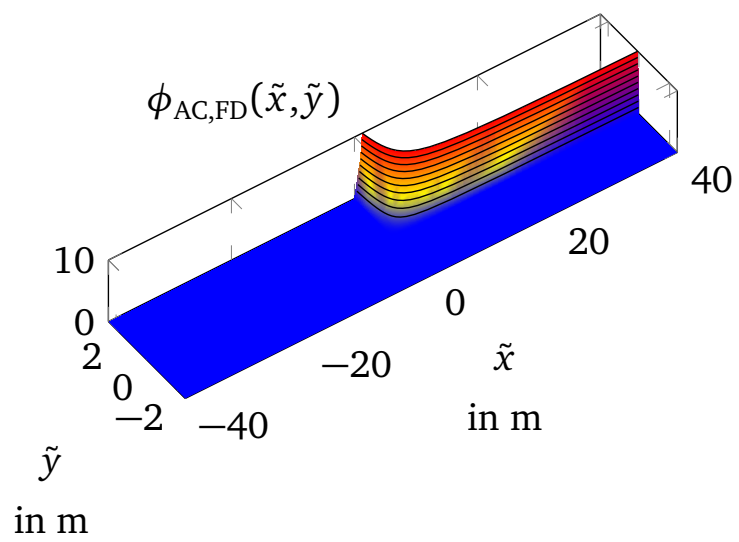

Abbildung 5.9: Potential zur Vermeidung von Kollisionen mit dem Fahrzeug FD

Da $\tilde{x}$ und $\tilde{y}$ von der eigenen Fahrzeugbewegung und den zeitabhängigen Umfeldobjektpositionen abhängig sind, können diese als Funktion von $t, p_{\mathrm{x}}$ und $p_{\mathrm{y}}$ ausgedrückt werden. Somit ergibt sich für den Güteterm für alle vier Fahrzeuge:

$$
J_{\mathrm{AC}, \mathrm{Cars}}=\int_{0}^{n T}\left[\sum_{c \in\{\mathrm{FS}, \mathrm{FD}, \mathrm{TS}, \mathrm{TD}\}} \phi_{\mathrm{AC}, \mathrm{c}}\left(t, p_{\mathrm{x}}(t), p_{\mathrm{y}}(t)\right)\right] \mathrm{d} t
$$

\section{Fahrstreifen}

Die Position der Umfeldfahrzeuge auf unbeteiligten Fahrstreifen (andere als Start- und Zielfahrstreifen) werden nicht innerhalb der Planung verarbeitet. Um Kollisionen mit diesen 
Fahrzeugen zu vermeiden, dürfen unbeteiligte Fahrstreifen nicht befahren werden. Um dies zu erreichen, wird eine abschnittsweise definierte Potentialfunktion $\phi_{\mathrm{AC} \text {,Lane }}$ verwendet, die eine Annäherung an andere Fahrstreifen bestraft (Abbildung 5.10):

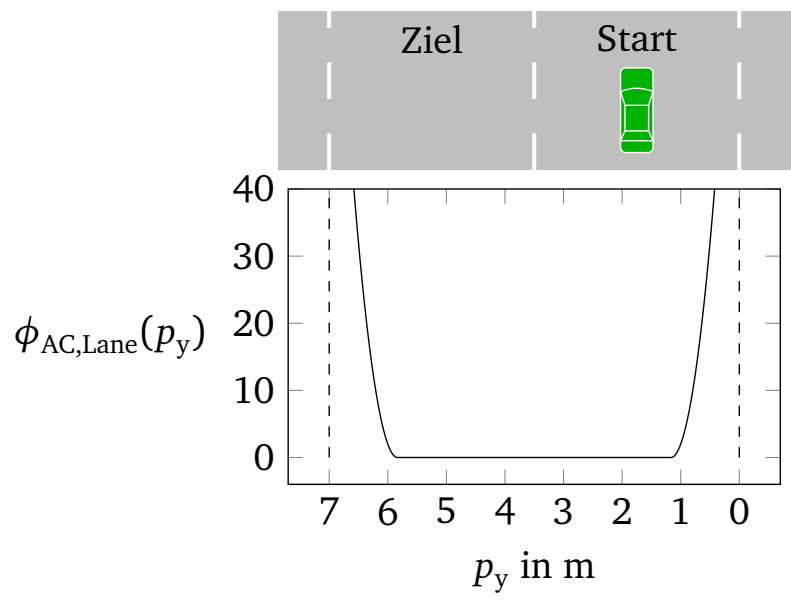

Abbildung 5.10: Potentialfunktion um zu vermeiden, dass unbeteiligte Fahrstreifen befahren werden

$$
\phi_{\text {AC,Lane }}=\left\{\begin{array}{lr}
36 \cdot\left(p_{\mathrm{y}}-p_{\text {AC,Lane,max }}\right)^{2} & p_{\mathrm{y}}>p_{\text {AC,Lane,max }} \\
0 & p_{\text {AC,Lane,min }} \geq p_{\mathrm{y}} \geq p_{\text {AC,Lane,max }} \\
36 \cdot\left(p_{\mathrm{y}}-p_{\text {AC,Lane,min }}\right)^{2} & p_{\text {AC,Lane,min }}>p_{\mathrm{y}}
\end{array}\right.
$$

Bei der Berechnung der Grenzen $p_{\text {AC,Lane,min }}$ und $p_{\text {AC,Lane,max }}$ wird die Fahrzeugbreite berücksichtigt. Abschließend erhält man den Strafterm $J_{\mathrm{AC} \text {,Lane }}$ wieder durch Integration über der Zeit:

$$
J_{\text {AC,Lane }}=\int_{0}^{n T} \phi_{\text {AC,Lane }}\left(p_{\mathrm{y}}(t)\right) \mathrm{d} t
$$

\section{Summe}

Der Gesamtstrafterm $J_{\mathrm{AC}}$ ergibt sich schließlich als gewichtete Summe von $J_{\mathrm{AC} \text {,Lane }}$ und $J_{\mathrm{AC} \text {,Cars }}$ :

$$
J_{\mathrm{AC}}=w_{\mathrm{AC}, \text { Lane }} \cdot J_{\mathrm{AC}, \text { Lane }}+w_{\mathrm{AC}, \text { Cars }} \cdot J_{\mathrm{AC} \text {, Cars }}
$$

\subsubsection{Einfluss auf den rückwärtigen Verkehr}

Für die Bewertung des Einflusses auf den rückwärtigen Verkehr wurde in einem ersten Ansatz ein ähnliches Verfahren wie bei der Drei-Segment-Planung (Abschnitt 4.5.2) verwendet. Zur Reduktion der Rechenzeit wurde jedoch eine vom Ego-Verhalten unabhängige Prädiktion des rückwärtigen Fahrzeugs gewählt (Abschnitt 5.5.1). Der Definitionsbereich des zur Bewertung 
verwendeten Intelligent-Driver-Models (IDM) wurde auf alle Quadranten erweitert. Diese Erweiterung war jedoch nicht stetig differenzierbar.

Diese Unstetigkeit hat zusammen mit dem $\frac{1}{x}$ Verhalten des IDMs bezüglich der Position (siehe S. 41) dazu geführt, dass der Optimierungsalgorithmus deutlich schlechter konvergierte. Die damit erzielten Ergebnisse waren nicht zufriedenstellend.

Aus diesem Grund wird die Bewertung des Einflusses auf den rückwärtigen Verkehr aktuell vom folgenden Abschnitt $J_{\mathrm{SA}}$ - Sicherheitsabstände mit abgedeckt. Sofern eine auf dem gesamten Suchraum stetig differenzierbare Funktion vorliegt, die das Verhalten des rückwärtigen Verkehrs beschreibt, wäre es sinnvoll, eine präzisere Prädiktion und Bewertung zu untersuchen. Aktuell gibt es jedoch keine Hinweise darauf, dass dies notwendig wäre.

\subsection{3 $J_{\mathrm{SA}}$ - Sicherheitsabstände}

Neben der Kollisionsvermeidung ist es erwünscht, gewisse Sicherheitsabstände zu den Umfeldfahrzeugen einzuhalten. Diese dürfen jedoch kurzzeitig verletzt werden, falls es erforderlich ist. Da die Spline-Planung dauerhaft aktiv ist und nicht an ein ACC übergeben wird, ist das im Folgenden beschriebene Potential auch für den Abstand zum vorderen Fahrzeug während der Folgefahrt zuständig. Die Vorgehensweise ähnelt dem der Kollisionsvermeidung, es werden jedoch andere Bereiche und Potentiale verwendet.

In Längsrichtung wird zunächst wieder für jedes Umfeldfahrzeug ein Bereich definiert (Abbildung 5.11).

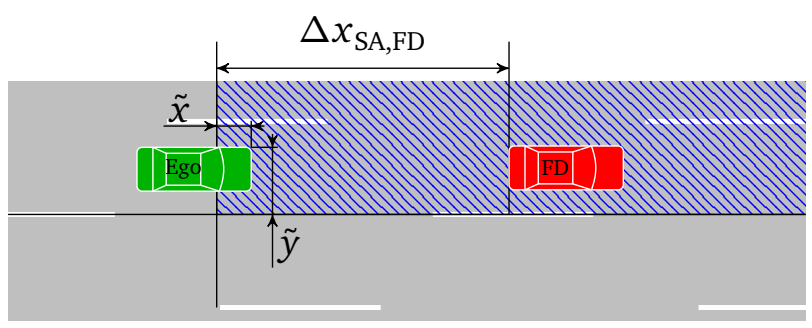

Abbildung 5.11: Bereich zum Einhalten des Sicherheitsabstandes zum Fahrzeug FD

Die Länge $\Delta x_{\mathrm{SA}, \mathrm{FD}}$ des Bereiches ist abhängig von der Geschwindigkeit des Umfeldfahrzeugs, jedoch größer als bei der Kollisionsvermeidung:

$$
\Delta x_{\mathrm{SA}, \mathrm{FD}}=\Delta T_{\mathrm{SA}, \mathrm{Front}} \cdot v_{\mathrm{FD}}+\Delta x_{0, \mathrm{SA}, \mathrm{Front}}
$$

- $\Delta T_{\mathrm{SA}, \mathrm{Front}}$ : Zeitlücke (geschwindigkeitsabhängiger Anteil)

- $\Delta x_{0, \mathrm{SA}, \text { Front }}:$ konstanter Term

Für jedes Umfeldfahrzeug $c \in\{F D, F S, T S, T D\}$ wird ein Potential $\phi_{\mathrm{SA}, c}$ definiert. Das Potential setzt sich jeweils aus verschiedenen multiplikativen Komponenten zusammen: Bewertung $f_{\mathrm{SA}, \mathrm{Lon}, c}$ der longitudinalen Eindringtiefe $\tilde{x}$, der Fahrstreifenbelegungsfunktion $f_{\mathrm{SA}, \mathrm{Lat}, c}$ in 
Querrichtung sowie, für die vorderen Umfeldfahrzeuge, $f_{\mathrm{SA}, \mathrm{vrel}, \mathrm{c}}$ zur Berücksichtigung der Relativgeschwindigkeit $v_{\text {rel }}$ gegenüber den vorderen Fahrzeugen. Das Gesamtpotential $\phi_{\mathrm{SA}}$ ist die Summe der vier Umfeldfahrzeugpotentiale:

$$
\begin{aligned}
\phi_{\mathrm{SA}} & =\sum_{c \in\{F D, F S\}}\left[f_{\mathrm{SA}, \mathrm{Lon}, c}(\tilde{x}) \cdot f_{\mathrm{SA}, \mathrm{Lat}, c}(\tilde{y}) \cdot f_{\mathrm{SA}, \mathrm{vrel}, c}\left(v_{\mathrm{rel}}\right)\right] \\
& +\sum_{c \in\{T D, T S\}}\left[f_{\mathrm{SA}, \mathrm{Lon}, \mathrm{c}}(\tilde{x}) \cdot f_{\mathrm{SA}, \mathrm{Lat}, c}(\tilde{y})\right]
\end{aligned}
$$

Betrachtet werden nun die einzelnen Komponenten am Beispiel von FD.

\section{$f_{\text {SA,Lon }}$ - Bewertung der longitudinalen Eindringtiefe}

Wie auch bei der Kollisionsvermeidung wird die Eindringtiefe $\tilde{x}$ quadratisch bestraft (Abbildung 5.12):

$$
f_{\mathrm{SA}, \mathrm{Lon}, F D}= \begin{cases}\tilde{x}^{2} & \tilde{x}>0 \\ 0 & \tilde{x} \leq 0\end{cases}
$$

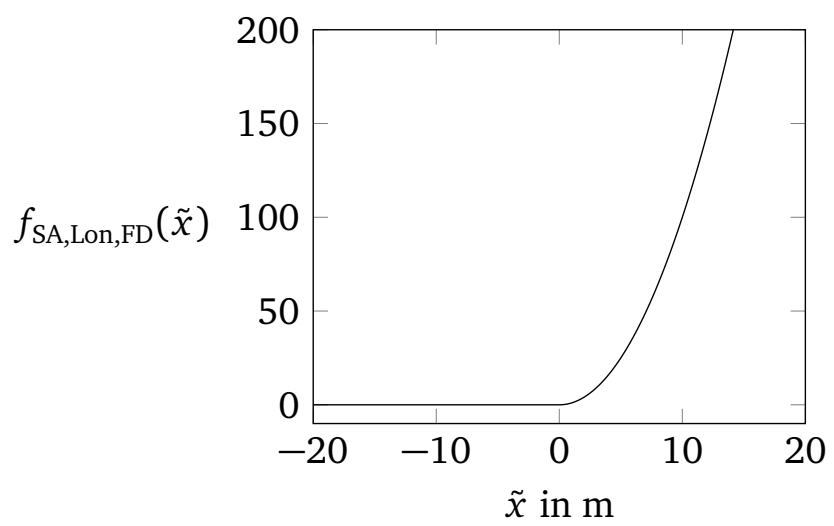

Abbildung 5.12: Quadratische Bestrafung der Eindringtiefe in Längsrichtung

\section{$f_{\text {SA,Lat }}-$ Fahrstreifenbelegungsfunktion}

Der Sicherheitsabstand zum Umfeldfahrzeug muss nur eingehalten werden, wenn dessen Fahrstreifen auch befahren wird. Für diese Unterscheidung sorgt die Fahrstreifenbelegungsfunktion $f_{\mathrm{SA}, \mathrm{Lat}, \mathrm{c}}$. Sie muss so aufgebaut sein, dass sie bei einer normalen Fahrt auf dem Fahrstreifen keine Abhängigkeit von der Querposition aufweist. Ansonsten käme es beim Bremsen auf ein vorderes Fahrzeug zu einer Ausweichbewegung in Querrichtung. Dies ist nicht gewünscht. Die Funktion wurde abschnittsweise aus quadratischen Funktionen zusammengesetzt. Sie startet $\Delta=0,5 \mathrm{~m}$ 
bevor das eigene Fahrzeug den betrachteten Fahrstreifen überdeckt und steigt an bis sie bei einer Überdeckung von 0,5 m ihren Maximalwert von 1 erreicht (Abbildung 5.13):

$$
f_{\mathrm{SA}, \mathrm{Lat}, \mathrm{FD}}(\tilde{y})=\left\{\begin{array}{lr}
1 & \tilde{y} \geq \Delta \\
1-a \cdot(\tilde{y}-\Delta)^{2} & \Delta>\tilde{y} \geq 0 \\
a \cdot(\tilde{y}+\Delta)^{2} & 0>\tilde{y} \geq \\
0 & -\Delta>\tilde{y}
\end{array} \quad \text { mit } \quad a=\frac{1}{2 \Delta^{2}}\right.
$$

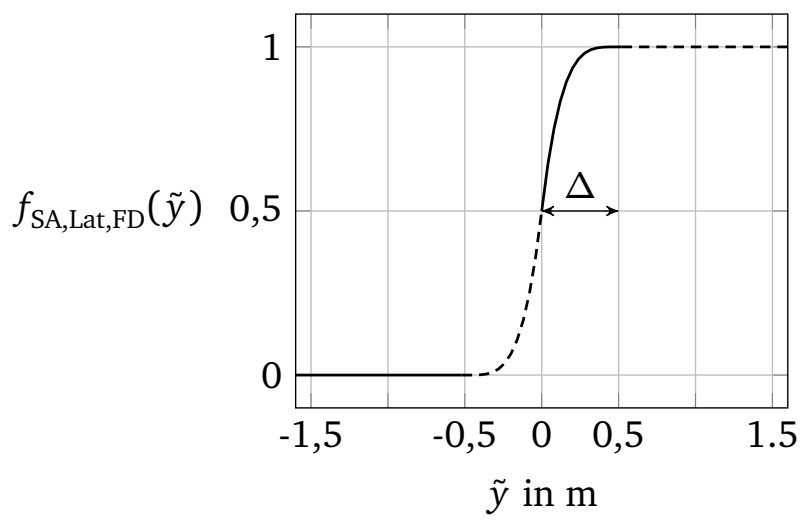

Abbildung 5.13: Fahrstreifenbelegungsfunktion für das Fahrzeug FD

\section{$f_{\mathrm{SA}, \mathrm{vrel}}-$ Berücksichtigung der Relativgeschwindigkeit}

In Fahrversuchen hat sich gezeigt, dass es Situationen gibt, in denen der Sicherheitsabstand nach Gleichung (5.17) als zu konservativ empfunden wird. Dies ist insbesondere dann der Fall, wenn hinter einem schnelleren Fahrzeug eingeschert werden soll, welches das EgoFahrzeug überholt. In diesem Fall ist es wünschenswert, dass das Fahrzeug den Wechsel schon beginnt, obwohl der Sicherheitsabstand noch nicht komplett eingehalten wird. Durch die Relativgeschwindigkeit ist dies auch gefahrlos möglich.

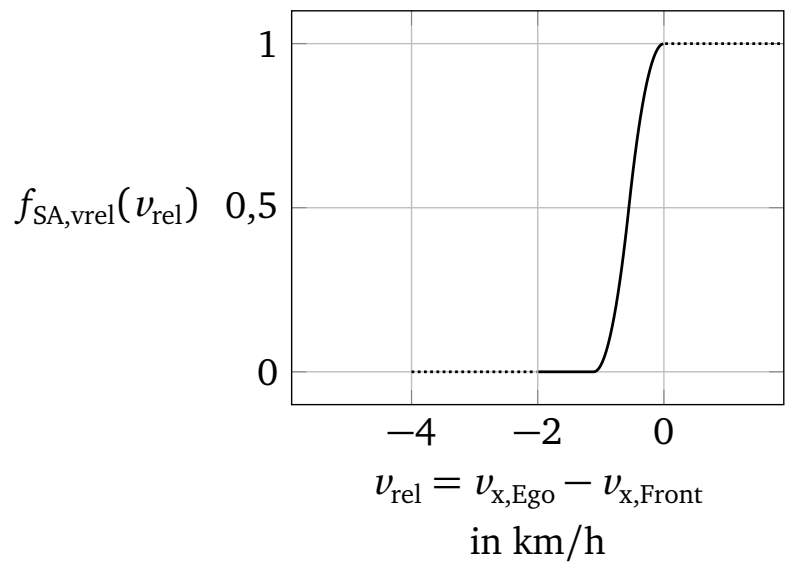

Abbildung 5.14: Relativgeschwindigkeitsabhängiger Faktor für die vorderen Fahrzeuge 
Im Rahmen dieser Arbeit wird das durch einen relativ einfachen zusätzlichen Faktor realisiert. Dieser ist im Wesentlichen eine Art Schalter, der den Sicherheitsbereich ab einer gewissen Relativgeschwindigkeit deaktiviert. Prinzipiell wäre hierfür aber auch eine fundiertere Funktion denkbar. Der Aufbau der Funktion ähnelt dabei sehr dem der Fahrstreifenbelegungsfunktion nach Gleichung (5.20), verwendet jedoch andere Übergangsbereiche (Abbildung 5.14).

\section{Gesamtpotential}

Werden die einzelnen Funktionen gemäß Gleichung (5.18) multiplikativ überlagert, so erhält man das Potential zur Einhaltung der Sicherheitsabstände, das in Abbildung 5.15 beispielhaft dargestellt ist.
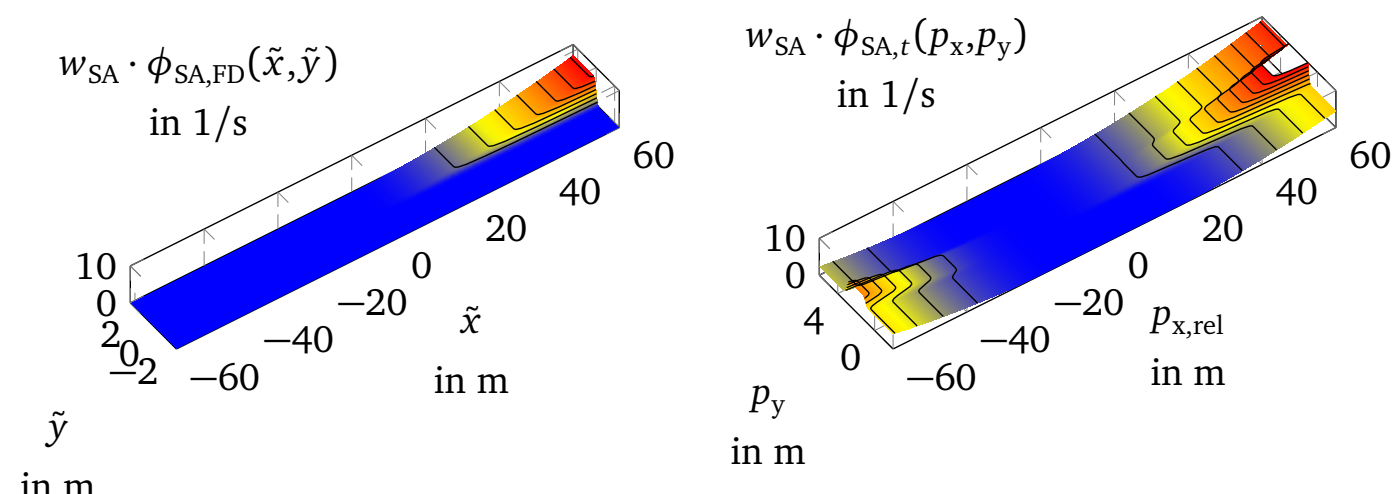

Abbildung 5.15: Beispiel für ein Potential zur Einhaltung der Sicherheitsabstände. Links zu FD (in Abhängigkeit der Größen $\tilde{x}$ und $\tilde{y}$ ), rechts zu allen vier Fahrzeugen (in Abhängigkeit der Größen $p_{\mathrm{x}}$ und $p_{\mathrm{y}}$ zu einem bestimmten Zeitpunkt)

Wie auch bei der Kollisionsvermeidung wird der Güteterm abschließend durch Integration bestimmt:

$$
J_{\mathrm{SA}}=\int_{0}^{n T} \phi_{\mathrm{SA}}\left(t, p_{\mathrm{x}}(t), p_{\mathrm{y}}(t), v_{\mathrm{x}}(t)\right) \mathrm{d} t
$$





Abbildung 5.16: Beispielhafte Fahrstreifenpotentialfunktion $\phi_{\mathrm{FSP}}\left(p_{\mathrm{y}}\right)$, die sich aus $\phi_{\mathrm{FSM}}\left(p_{\mathrm{y}}\right)$ und $\phi_{\mathrm{FS}}\left(p_{\mathrm{y}}\right)$ zusammensetzt

Das Fahrstreifenpotential $\phi_{\text {FSP }}\left(p_{\mathrm{y}}\right)$ setzt sich aus zwei Komponenten zusammen (Abbildung 5.16):

$$
\phi_{\mathrm{FSP}}\left(p_{\mathrm{y}}\right)=w_{\mathrm{FSM}} \cdot \phi_{\mathrm{FSM}}\left(p_{\mathrm{y}}\right)+w_{\mathrm{FS}} \cdot \phi_{\mathrm{FS}}\left(p_{\mathrm{y}}\right)
$$

Die erste Komponente $\phi_{\mathrm{FSM}}\left(p_{\mathrm{y}}\right)$ bestraft quadratisch die Abweichung von der Zielfahrstreifenmitte. Somit sorgt sie für einen zügigen Beginn des Fahrstreifenwechsels. Bei der zweiten Komponente $\phi_{\mathrm{FS}}\left(p_{\mathrm{y}}\right)$ handelt es sich um eine abschnittsweise definierte Funktion aus Polynomen zweiten Grades. Ihre Form passt sich den unterschiedlich breiten Fahrstreifen an. Durch sie wird ein unnötig langes Verweilen zwischen den Fahrstreifen vermieden. Durch Variation des Gewichtungsfaktors $w_{\mathrm{FS}}$ kann daher die Maximaldauer des Fahrstreifenwechsels verändert werden.

Durch die Überlagerung der beiden Komponenten entsteht eine Funktion, deren Nullpunkt und globales Minimum in der Mitte des Zielfahrstreifens liegt und die lokale Minima auf den einzelnen Fahrstreifen besitzt (Abbildung 5.16). Die in Abschnitt 5.2 beschriebene Endpunktbewertung in Querrichtung sorgt dafür, dass die Trajektorie, trotz des Potentialberges zwischen den Fahrstreifen, auf dem Zielfahrstreifen endet. Bei der gewählten hohen Gewichtung der Endpunktbewertung lag der Endpunkt in der Praxis immer auf dem Zielfahrstreifen. Trotzdem kann dies nicht grundsätzlich garantiert werden. Besser wäre es, den Endpunkt im Rahmen einer alternativen Parametrierung fest vorzugeben (siehe auch Abschnitt 6.3 und Anhang B.2).

Bei der Approximation des Integrals

$$
J_{\mathrm{FSP}}=\int_{0}^{n T} \phi_{\mathrm{FSP}}\left(p_{\mathrm{y}}(t)\right) \mathrm{d} t
$$


kann es jedoch leicht zu numerisch bedingten Nebenminima kommen. Diese können zu einem unnötigen Herauszögern des Fahrstreifenwechsels führen. Um diese Nebenminima zu vermeiden, wird zur Bestimmung des Integrals ein spezielles Verfahren verwendet. Hierbei wird die Trajektorie zwischen den Abtastpunkten linear approximiert. Für diesen stückweise linearen Verlauf wird anschließend das Integral analytisch mit Hilfe der Stammfunktion berechnet. Der Aufbau des Potentials sowie das Integrationsverfahren sind in Anhang B.4 näher beschrieben.

\subsubsection{Summenpotential}

Für ein besseres Verständnis der Potentialfunktionen wird in Abbildung 5.17 ein gewichtetes Summenpotential

$$
\phi_{\mathrm{Sum}}=\phi_{\mathrm{AC}, \mathrm{Lane}} \cdot w_{\mathrm{AC}, \mathrm{Lane}}+\phi_{\mathrm{AC}, \mathrm{Cars}} \cdot w_{\mathrm{AC}, \mathrm{Cars}}+\phi_{\mathrm{SA}} \cdot w_{\mathrm{SA}}+\phi_{\mathrm{FSP}}
$$

dargestellt.

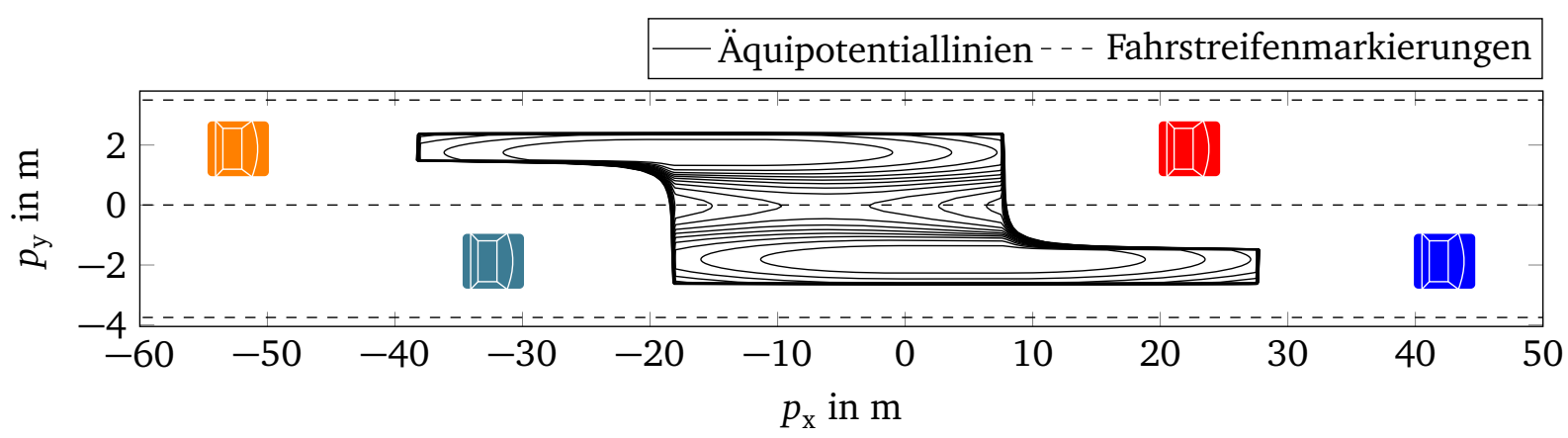

Abbildung 5.17: Äquipotentiallinien des Summenpotentials $\phi_{\text {Sum }}$

Am Rand des befahrbaren Bereiches steigt das Potential bedingt durch die Kollisionsvermeidung steil an. Auf der Fahrstreifenmarkierung erkennt man den Einfluss des Fahrstreifenpotentials, und in den Mitten von Start- und Zielfahrstreifen sieht man den Einfluss des Potentials zur Einhaltung der Sicherheitsabstände. Es ist Aufgabe der Trajektorienplanung, einen möglichst günstigen Weg aus dem lokalen Minimum auf dem Startfahrstreifen ins globale Minimum auf dem Zielfahrstreifen zu wählen. Dass sich der Endpunkt auf dem Zielfahrstreifen befindet, wird durch die Endpunktbewertung der Quertrajektorie forciert. Bei der Planung der Trajektorie sind noch weitere Bedingungen zu berücksichtigen, die im Folgenden beschrieben werden.

\subsection{6 $J_{\mathrm{ED}}$ Beschränkung der Trajektoriendynamik}

Um sicherzustellen, dass das Fahrzeug der Trajektorie folgen kann, muss deren Dynamik begrenzt werden. Analog zur Drei-Segment-Planung (Abschnitt 4.5.3) werden Geschwindigkeit 
und Beschleunigung in Längs- und Querrichtung mit Schwellwerten verglichen und deren Übertretung quadratisch bestraft:

$$
\begin{aligned}
J_{\mathrm{ED}} & =\int_{0}^{n T}\left[f_{\mathrm{v}, \text { Lon }}\left(v_{\mathrm{Lon}}(t)\right)\right] \mathrm{d} t+\int_{0}^{n T}\left[f_{\mathrm{a}, \text { Lon }}\left(a_{\mathrm{Lon}}(t)\right)\right] \mathrm{d} t \\
& +\int_{0}^{n T}\left[f_{\mathrm{v}, \text { Lat }}\left(v_{\mathrm{Lat}}(t)\right)\right] \mathrm{d} t+\int_{0}^{n T}\left[f_{\mathrm{a}, \text { Lat }}\left(a_{\mathrm{Lat}}(t)\right)\right] \mathrm{d} t
\end{aligned}
$$

Hierbei kommen allerdings andere Schwellwerte zum Einsatz, die in Tabelle 5.2 aufgeführt sind. Es wurden die gleichen Gewichtungen für die einzelnen Größen verwendet wie bei der Drei-Segment-Planung. Aus Gründen der Vollständigkeit sind sie in der Tabelle noch einmal aufgeführt.

Tabelle 5.2: Übersicht der Schwellwerte und Gewichtungsfaktoren zur Beschränkung der

\begin{tabular}{|c|c|c|c|c|c|c|c|}
\hline Größe & $\min$ & $\max$ & Einheit & Gewicht & $k_{\ldots, \min }$ & $k_{\ldots, \max }$ & Einheit \\
\hline$\nu_{\text {Lat }}$ & -2 & 2 & $\mathrm{~m} / \mathrm{s}$ & $k_{\mathrm{Lat}, \mathrm{v}}$ & 100 & 100 & $\mathrm{~s} / \mathrm{m}^{2}$ \\
\hline$a_{\text {Lat }}$ & $-2,8$ & 2,8 & $\mathrm{~m} / \mathrm{s}^{2}$ & $k_{\mathrm{Lat}, \mathrm{a}}$ & 100 & 100 & $\mathrm{~s}^{3} / \mathrm{m}^{2}$ \\
\hline$v_{\text {Lon }}$ & 0,01 & $v_{\mathrm{ACC}, \mathrm{Setz}}$ & $\mathrm{m} / \mathrm{s}$ & $k_{\text {Lon,v }}$ & 10 & 0,01 & $\mathrm{~s} / \mathrm{m}^{2}$ \\
\hline$a_{\mathrm{Lon}}$ & -3 & $a_{\max , \mathrm{PSP}}(v)$ & $\mathrm{m} / \mathrm{s}^{2}$ & $k_{\text {Lon,a }}$ & 100 & 100 & $\mathrm{~s}^{3} / \mathrm{m}^{2}$ \\
\hline
\end{tabular}
Trajektoriendynamik bei der Spline-Planung

Ein besonderes Augenmerk muss auf die geschwindigkeitsabhängige Maximalbeschleunigung $a_{\text {max,PSP }}(v)$ gelegt werden (Abbildung 5.18). Diese Abhängigkeit muss bei der Gradientenberechnung berücksichtigt werden.

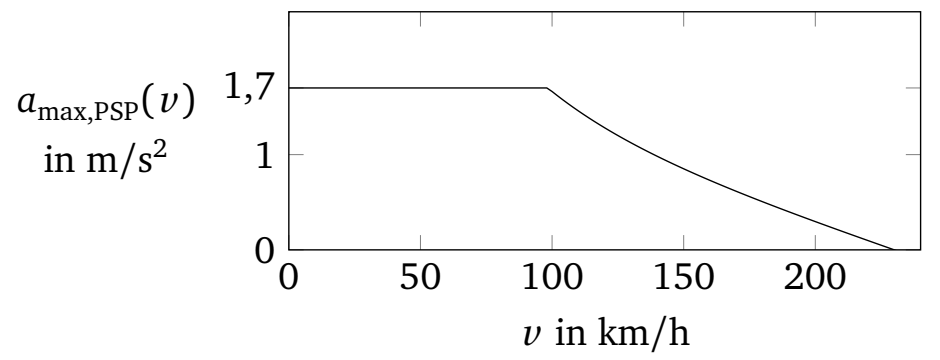

Abbildung 5.18: Maximale Längsbeschleunigung der Spline-Planung in Abhängigkeit der Geschwindigkeit

\subsubsection{Bewertung des Endpunktes in Längsrichtung}

Die Bewertung des Endpunktes der Trajektorie besteht aus zwei Teilen:

- Vorankommen belohnen

- Überschüssige Energie am Endpunkt bestrafen 


\section{Vorankommen belohnen}

Im Teil für das Vorankommen gehen Endgeschwindigkeit sowie Endposition linear mit negativem Gewicht in die Straffunktion ein. Eine Minimierung der Straffunktion führt so zu einer Maximierung der beiden Größen:

$$
J_{\text {Lon,End,Forward }}=w_{\text {Lon,End, } \mathrm{p}} \cdot p_{\mathrm{x}, \mathrm{e}}+w_{\text {Lon,End, } \mathrm{v}} \cdot v_{\mathrm{x}, \mathrm{e}}
$$

Im Gegensatz zur Drei-Segment-Trajektorie besitzt die Spline-Trajektorie eine konstante zeitliche Länge. Daher ist es hier nicht notwendig, die Endposition in Relation zur Endzeit zu bewerten. Auf die Verwendung einer Durchschnittsgeschwindigkeit, wie bei der Drei-SegmentPlanung (Abschnitt 4.5.4, Gl. 4.27), kann daher hier verzichtet werden.

\section{Überschüssige Energie}

Der zweite Teil verhindert, dass sich das Ego-Fahrzeug einem vorausfahrenden mit unnötig hoher Geschwindigkeit nähert. Ohne ihn könnte es zu dem paradoxen Effekt kommen, dass das Ego-Fahrzeug in Folgefahrt immer weiter verzögert und einen immer größeren Abstand zum vorausfahrenden Fahrzeug hält. Dadurch kann eine Trajektorie geplant werden, die stark beschleunigt und am Trajektorienende eine sehr hohe Geschwindigkeit erreicht.

Um dieses Verhalten zu verhindern, ist eine plausible Endpunktbewertung wichtig. Zudem sollte die Funktion nicht unnötig extrem auf kleine Abweichungen in der Endgeschwindigkeit und -position reagieren. Ungeeignet sind beispielsweise folgende Maße:

- „Verzögerung, die notwendig ist, um den Sicherheitsabstand nicht zu verletzen“. Befindet sich das Fahrzeug kurz vor dem Sicherheitsabstand aber mit einer höheren Geschwindigkeit, so müssen extreme Verzögerungen aufgebracht werden, um den Sicherheitsabstand nicht zu verletzten.

- „Beschleunigung basierend auf dem Intelligent-Driver-Model“. Befindet sich der Endpunkt vor dem vorausfahrenden Fahrzeug, so werden extreme Werte ausgegeben oder die Funktion ist nicht definiert.

Um dieses Problem zu vermeiden, wurde folgendes Maß verwendet: „Überschüssige Energie, die noch abgebaut werden müsste, um dann durch Ausrollen den Sicherheitsabstand zu erreichen“. Hierzu wird für den Trajektorienendpunkt eine Sollgeschwindigkeit berechnet, die das eigene Fahrzeug aufweisen müsste, so dass es durch Ausrollen den Sicherheitsabstand zum vorausfahrenden Fahrzeug erreicht. Aus dem Vergleich dieser Sollgeschwindigkeit mit der eigenen Geschwindigkeit am Endpunkt lässt sich die überschüssige Energie am Endpunkt bestimmen. Um die Konvergenz der Optimierung nicht zu gefährden, ist es notwendig, auch für Endpunkte, die sich im Sicherheitsabstand befinden, sinnvolle Werte auszugeben. Für Endpunkte im Sicherheitsabstand wird daher eine Sollgeschwindigkeit vorgegeben, die kleiner ist als die des vorausfahrenden Fahrzeugs. Die zur Berechnung der Differenzenergie notwendigen Funktionen weisen dabei einige Stellen auf, die nicht stetig differenzierbar sind. 
Um trotzdem eine stetig differenzierbare Gütefunktion zu erhalten, wurden die Funktionen an diesen Stellen entsprechend angepasst. Die Details dazu befinden sich in Anhang B.3.

Die gesamte Endpunktbewertung erhält man anschließend durch Addition:

$$
J_{\text {Lon,End }}=J_{\text {Lon,End,Forward }}\left(p_{\mathrm{x}}, v_{\mathrm{x}}\right)+w_{\text {Lon,End,Loss }} \cdot J_{\text {Lon,End,Loss }}\left(p_{\mathrm{x}}, v_{\mathrm{x}}\right)
$$

Die Werte der Parameter sind in Tabelle 5.3 aufgeführt.

Tabelle 5.3: Werte der Parameter zur Endpunktbewertung

\begin{tabular}{lcc} 
Parameter & Wert & Einheit \\
\hline$w_{\text {End,p }}$ & $-0,015$ & $1 / \mathrm{m}$ \\
$w_{\text {End,v }}$ & $-0,007$ & $\mathrm{~s} / \mathrm{m}$ \\
$w_{\text {End,Loss }}$ & 0,15 & $\mathrm{~s}^{4} / \mathrm{m}^{2}$
\end{tabular}

\subsection{Summation der Straffunktionen}

Nach Bewertung eines Trajektorienkandidaten nach den unterschiedlichen Kriterien werden die einzelnen Strafterme gewichtet und addiert. Teilweise enthalten die Strafterme die Gewichtungen schon intern, teilweise werden sie in diesem Schritt gewichtet. Die verwendeten Werte der Gewichtungsparameter sind in Tabelle 5.4 aufgeführt.

$$
\begin{aligned}
& J_{\text {Ges }}=J_{\text {Lat }} \quad \text { Komfort in Querrichtung } \\
& +J_{\text {Lat,End }} \quad \text { Endpunktbewertung Querrichtung } \\
& +J_{\text {Lon }} \quad \text { Komfort in Längsrichtung }
\end{aligned}
$$

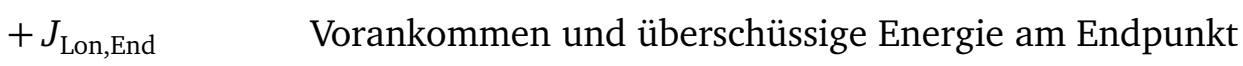

$$
\begin{aligned}
& +J_{\text {EgoDyn }} \quad \text { Beschränkung der Trajektoriendynamik } \\
& +J_{\mathrm{AC}, \text { Lane }} \cdot w_{\mathrm{AC}, \text { Lane }} \quad \text { Verlassen der Fahrstreifen } \\
& +J_{\mathrm{AC}, \text { Cars }} \cdot w_{\mathrm{AC} \text {,Cars }} \quad \text { Kollisionsvermeidung } \\
& +J_{\mathrm{SA}} \cdot w_{\mathrm{SA}} \quad \text { Sicherheitsabstände } \\
& +J_{\mathrm{FSP}} \quad \text { Fahrstreifenpotential }
\end{aligned}
$$

Zusammen mit der Gesamtgüte wird auch der Gradient hierfür analytisch berechnet. Anschließend wird der Gradient bzgl. der Abtastpunkte noch mit Hilfe der Gleichung (5.9) in Abschnitt 5.3 auf die Optimierungsvariablen $\mathbf{j}$ umgerechnet und dem Optimierungsalgorithmus zur Verfügung gestellt. 
Tabelle 5.4: Verwendete Werte der Gewichtungsparameter zur Summation der Güteterme bei der Spline-Planung

\begin{tabular}{llc} 
Parameter & Wert & Einheit \\
\hline$w_{\text {AC,Lane }}$ & 10 & $1 / \mathrm{s}$ \\
$w_{\text {AC,Cars }}$ & 10 & $1 / \mathrm{s}$ \\
$w_{\text {SA }}$ & 0,002 & $1 / \mathrm{s}$
\end{tabular}

\subsection{Bestimmung der optimalen Trajektorie}

Mit Hilfe eines iterativen nichtlinearen Optimierungsalgorithmus werden nun die optimalen Parameter für die Splines gesucht, die zu einer Minimierung der Gesamtgüte $J_{\text {Ges }}$ führen.

Die Verwendung des Downhill-Simplex-Verfahrens ist aufgrund der vielen Optimierungsvariablen hier nicht sinnvoll. Für die Spline-Planung wird daher das Quasi-Newton-Verfahren zur Optimierung verwendet. Die Hesse-Matrix wird hierbei durch den L-BFGS Algorithmus approximiert (L-BFGS: Limited-memory Broyden-Fletcher-Goldfarb-Shanno).

Für die Verwendung im Versuchsfahrzeug war eine MAтLAв-Implementierung erforderlich. Um den Algorithmus nicht selbst implementieren zu müssen, wurde auf die Funktion minFunc von Mark Schmidt [Schmidt, 2005] zurückgegriffen. Diese ist OpenSource und steht unter einer „FreeBSD-style“ Lizenz ${ }^{1}$. Die Implementierung wurde so angepasst, dass sie zur Codegenerierung geeignet ist.

Als Startlösung wird der Parametervektor des letzten Planungsschrittes übernommen. Hierbei wird zurzeit nicht berücksichtigt, dass die neu zu planende Trajektorie einen anderen Startzustand besitzt. Für die Berücksichtigung dieser Tatsache wäre es vorteilhaft, Positionen anstatt des Rucks als Optimierungsvariablen zu verwenden (Anhang B.2). Ist keine letzte Lösung für die entsprechende Lücke vorhanden, so wird $\underline{\mathbf{j}}=\underline{\mathbf{0}}$ als Startlösung verwendet.

Zur Beschränkung der Rechenzeit für einen Planungsschritt wurde die Anzahl der Funktionsauswertungen/ Trajektorienkandidaten auf 150 begrenzt.

Um zu erreichen, dass der Spline seinen Endpunkt auf dem Zielfahrstreifen hat, wird eine sehr hohe Quer-Endpunktgewichtung verwendet. Im Laufe der Tests hat sich jedoch gezeigt, dass dies aufgrund von numerischen Beschränkungen der Genauigkeit dazu führen kann, dass es Probleme gibt, das Optimum zu erreichen. Aus diesem Grund wird eine sehr hohe Endpunktbewertung zurzeit nur in den Fällen verwendet, in denen die Startlösung nicht auf dem Zielfahrstreifen endet. Im Normalfall wird eine nicht so starke Endpunktgewichtung verwendet. Langfristig wäre eine Lösung vorzuziehen, die diese Unterscheidung nicht benötigt. Zwei Lösungen wären hierfür zu untersuchen:

1 http://www.cs.ubc.ca/ schmidtm/Software/copyright.html 
- Den Endpunkt der Trajektorie fest vorzugeben.

- Positionen anstatt die Rucke als Optimierungsvariablen zu verwenden.

Abbildung 5.19 zeigt die ausgewerteten Trajektorienkandidaten während eines Planungsschrittes bzw. Optimierungsdurchlaufes. Als Startlösung wurde hierzu $\underline{\mathbf{j}}=\underline{\mathbf{0}}$ verwendet.

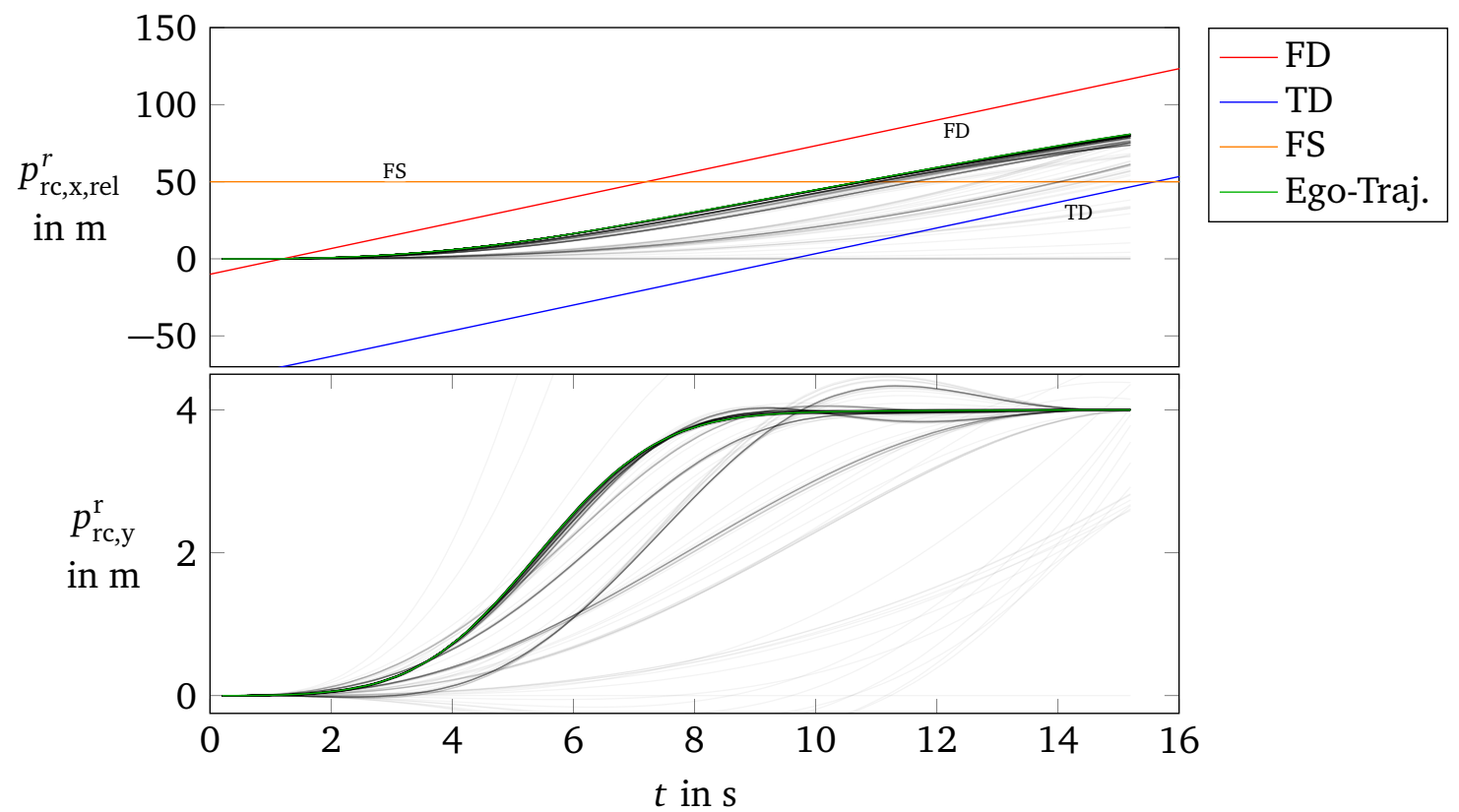

Abbildung 5.19: Ausgewertete Trajektorienkandidaten während eines Optimierungsdurchlaufes bzw. Planungsschrittes der Spline-Planung in Grau. Die beste und somit finale Trajektorie ist in Grün dargestellt. (Startwert: $\underline{\mathbf{j}}=\underline{\mathbf{0}}$ )

\subsection{Ergebnisse}

Auch die Spline-Planung wurde sowohl in der Simulation als auch im realen Verkehr getestet. Sie ermöglicht ebenfalls sichere und komfortable Fahrstreifenwechsel auch in dichtem Verkehr. Durch die bessere Ausnutzung der Beschleunigungsmöglichkeiten können sogar noch mehr Lücken angefahren werden, als dies bei der Drei-Segment-Planung möglich ist. Bei den Fahrten im realen Verkehr hat sich insbesondere die Notwendigkeit zur Berücksichtigung der Relativgeschwindigkeit gezeigt (vgl. Abschnitt 5.6.3). Weitere Ergebnisse der Praxistests sowie ein Vergleich mit der Drei-Segment-Planung befinden sich in Kapitel 6.

Abbildung 5.20 zeigt den sich ergebenden Sollzustandsverlauf bei einem Fahrstreifenwechsel mit realen Fahrzeugen auf dem Testgelände. In Grau sind die im Verlauf des Wechsels geplanten Trajektorien dargestellt. Wie erkennbar ist, wird ein stetiger Verlauf von Position, Geschwindigkeit und Beschleunigung erreicht. Aufgrund der langsamen Dynamik der Längsaktorik fiel der leicht eckige Verlauf der Beschleunigung bei den Testfahrten nicht negativ auf. Die Streuung der Trajektorien hat ihre wesentliche Ursache in einem Rauschen der Umfeldsensorik, wird jedoch bei der Fahrt nicht bemerkt, da immer nur der erste Teil einer Trajektorie abgefahren wird. 




Abbildung 5.20: Verlauf des an die Regelung vorgegebenen Sollzustandes während eines realen Fahrstreifenwechsels nach links zwischen zwei schnellere Fahrzeuge. In Grau sind alle zwischenzeitlich geplanten Trajektorien dargestellt.

\subsection{Rechenzeit}

Auch für die Spline-Planung wurde die grob zu erwartende Auslastung des Steuergerätes bestimmt. Dies geschah basierend auf den Daten der Drei-Segment-Planung sowie einem Vergleich der Berechnungszeiten auf dem Messrechner (Abschnitt 6.2.3). Ergebnis des Vergleichs ist die Tatsache, dass die Berechnung einer Spline-Trajektorie etwa dreimal so viel Zeit benötigt wie die einer Drei-Segment-Trajektorie. Es wird daher davon ausgegangen, dass die Berechnung einer Spline-Trajektorie unter Annahme einer optimalen Implementierung auf dem Steuergerät ca. 1,2 ms benötigt. Basierend auf diesen Daten läge die Auslastung des Steuergerätes bei der Berechnung von 5 Trajektorien bei etwa $3 \%$. 


\section{Ergebnisse \& Vergleich}

In diesem Kapitel werden zunächst die Ergebnisse der Praxistests der beiden Trajektorienplanungen vorgestellt. Anschließend werden die Verfahren auf Gemeinsamkeiten und Unterschiede untersucht. Am Ende werden mögliche Erweiterungen beschrieben und eine Empfehlung über den weiterzuverfolgenden Ansatz abgegeben.

\subsection{Praxistests}

Beide Trajektorienplanungen wurden mit Hilfe von MatLab/Simulink und CarMaker in unterschiedlichsten Situationen simulativ getestet. Neben einfacheren Szenarien wurden insbesondere folgende Fälle geprüft:

- Wechsel nach links zwischen zwei schneller fahrende Fahrzeuge

- Wechsel nach rechts zwischen zwei langsamere Fahrzeuge

- Abbruch eines Fahrstreifenwechsels, da der Zielfahrstreifen plötzlich von einem anderen Fahrzeug belegt wird

- Abbruch eines Fahrstreifenwechsels, da ein anderes Fahrzeug plötzlich bremst oder beschleunigt.

- Fahrstreifenwechsel in eine langsamere Lücke, bei dem von hinten ein schnelleres Fahrzeug drängelt.

Die Trajektorienplanungen mussten hierbei zeigen, dass sie den Fahrstreifenwechsel in diesen Situationen sicher durchführen können. Die größte Herausforderung bei der simulativen Erprobung ist die Modellierung des umgebenden Verkehrs. So reagieren Fahrer beispielsweise im realen Straßenverkehr häufig schon auf das Setzen eines Blinkers.

In der ersten verwendeten Version 4.0 von CarMaker konnte die Bewegung des Verkehrs nur vorab fest vorgegeben werden. Eine Reaktion der Umfeldfahrzeuge auf andere Fahrzeuge war dort nicht vorgesehen. Aus diesem Grund wurde im Rahmen einer Bachelorarbeit [Katke, 2014] eine einfache Simulation auf Basis des Intelligent-Driver-Models [Treiber, 2010, S. 162 f.] erstellt. Im weiteren Verlauf der Arbeit konnte auf die in CarMaker 4.5 vorhandene Umfeldsimulation zurückgegriffen werden. Diese ist jedoch ebenfalls relativ einfach aufgebaut und auf die Längsrichtung beschränkt.

Aus diesem Grund wurden die Trajektorienplanungen in einem Versuchsträger implementiert und immer wieder in Testfahrten erprobt. Diese wurden primär auf dem August-Euler-Flugplatz in Griesheim in der Nähe von Darmstadt durchgeführt. Die etwa einen Kilometer lange 
Startbahn diente hierbei, ausgestattet mit entsprechenden Fahrstreifenmarkierungen, als Autobahnnachbildung. Eine Reihe anderer echter Fahrzeuge simulierten den umgebenden Verkehr. Dabei wurden sowohl Fahrstreifenwechsel in unterschiedlichen Situationen durchgeführt, als auch deren Abbruch provoziert. Der Abbruch wurde im Wesentlichen auf zwei verschiedene Weisen provoziert. Im ersten Fall wechselte ein Fahrzeug während des Fahrstreifenwechsels von einem dritten Fahrstreifen auf Höhe des eigenen Fahrzeugs auf den Zielfahrstreifen. Im zweiten Fall beschleunigte das rückwärtige Fahrzeug auf dem Zielfahrstreifen. Beide Trajektorienplanungen waren dabei in der Lage, diese Szenarien erfolgreich zu durchfahren.

Da die Fahrer bei diesen Tests aufgrund der beschränkten zur Verfügung stehenden Strecke in die Situation eingeweiht sein mussten, verhielten sie sich ggf. abweichend vom realen Straßenverkehr. Um dem zu begegnen, fanden auch Tests auf der Autobahn statt. Primär geschah dies auf der A81 nahe Abstatt sowie in der Umgebung von Darmstadt. Der Versuchsträger wies leider zwei Einschränkungen auf: Der Fahrer musste manuell den richtigen Gang wählen, da das Automatikgetriebe nicht selbstständig herunterschaltete. Des Weiteren kam es gelegentlich trotz der in Anhang F.3 beschriebenen Verbesserungen zu einer fehlerhaften Fahrstreifenzuordnung einiger Umfeldfahrzeuge. Aus diesem Grund wurde keine Probandenstudie durchgeführt und das Fahrzeug auf der Autobahn ausschließlich durch am Projekt beteiligte Bosch-Mitarbeiter gefahren. Bei den Testfahrten war erkennbar, dass die Spline-Planung in der Lage ist, die Beschleunigungsmöglichkeiten des Fahrzeugs besser auszunutzen. So kann sie noch Lücken nutzen, die mit der Drei-Segment-Planung nicht mehr erreichbar sind.

Die Ergebnisse der Versuchsfahrten wurden detailliert ausgewertet. Während der Versuchsfahrten wurden dazu, neben den Sensordaten und den geplanten Trajektorien, die Daten einer Front- und Heck-Kamera aufgezeichnet. Im Anschluss an die Versuchsfahrten konnte dann mit einem selbst entwickelten MaTLAB-Tool jeder einzelne Planungsschritt zusammen mit der Umfeldsituation analysiert werden. Die daraus gewonnenen Erkenntnisse waren bei der Entwicklung der Trajektorienplanung sehr hilfreich.

Insgesamt sind mit der Drei-Segment-Planung mehr als $800 \mathrm{~km}$ und mit der SplinePlanung mehr als $500 \mathrm{~km}$ an Testfahrten auf der Autobahn absolviert worden. Beide Trajektorienplanungen können dabei auch in dichtem Verkehr komfortable Fahrstreifenwechsel durchführen. Weiterhin sind beide in der Lage, einen Fahrstreifenwechsel abzubrechen, sofern die Situation dies erfordert.

\section{Subjektive Eindrücke der Fahrtests während der Abschlusspräsentation}

Im Rahmen der Abschlusspräsentation wurde das System sowohl auf dem Testgelände in Griesheim als auch auf der Autobahn in der Umgebung von Darmstadt getestet. Dabei wurden die Fahrstreifenwechsel von den Fahrern als gut, komfortabel und sehr angenehm beschrieben. Der Wechsel in eine langsamere Lücke wird als komfortabel empfunden. Es wurde jedoch gewünscht, dass Verzögerung und Fahrstreifenwechsel etwas früher einsetzen. Insbesondere 
auf der Autobahn wäre teilweise ein schnelleres Herausziehen und Hereinfahren in die Lücke wünschenswert.

Der Unterschied zwischen den beiden Planungen war für manche Teilnehmer kaum zu spüren. Andere Teilnehmer fanden die Drei-Segment-Planung spontaner, aber auch etwas ruppiger. Es wurde bemerkt, dass die Spline-Planung früher auf den Zielfahrstreifen wechselt.

Die Abbruchmanöver wurden als gut empfunden, wobei die Spline-Planung bei den auf dem Flugplatz gestellten, relativ kritischen Szenarien harmonischer wirkte.

\subsection{Vergleich}

Zum Vergleich der beiden Trajektorienplanungen werden erst allgemeine Gemeinsamkeiten und Unterschiede im Aufbau der beiden Planungen betrachtet. Eine Gegenüberstellung der entstehenden Trajektorien befindet sich dann in Abschnitt 6.2.1. Anschließend wird die Konvergenz der Verfahren (Abschnitt 6.2.2) sowie die benötigten Berechnungszeiten (Abschnitt 6.2.3) verglichen. Abschließend wird auf die Stärken und Schwächen der beiden Planungen eingegangen.

Beim Vergleich der beiden Trajektorienplanungen entdeckt man eine Reihe an Gemeinsamkeiten, aber auch Unterschiede. So ergibt sich die Trajektorie bei beiden Planungen aus der Lösung eines nichtlinearen Optimierungsproblems. Die Gütefunktionen der beiden Planungen ähneln einander, weisen jedoch einige Unterschiede auf.

Die Gütefunktion der Drei-Segment-Planung weist an diversen Stellen, insbesondere durch die Generierung (Abschnitt 4.3), maßgebliche Nichtlinearitäten auf. Hierzu gehört zum einen die Bestimmung der Ein- und Austrittspunkte bezüglich der Wechsellücke, aber auch die Abhängigkeit der Matrizen $\underline{\mathbf{W}}_{\mathrm{G} 1}$ bis $\underline{\mathbf{W}}_{\mathrm{G} 3}$ sowie $\underline{\mathbf{C}}$ und $\underline{\mathbf{d}}$ von diesen Ein- und Austrittspunkten (siehe Anhang A.1). Zur Optimierung der maximal drei Parameter $\left(\bar{v}_{1}, \bar{v}_{2}, v_{\mathrm{e}}\right)$ wird der Downhill-Simplex-Algorithmus verwendet. Dieser kommt ohne Gradienteninformation aus. Daher wurde bewusst auf eine stetig differenzierbare Gütefunktion verzichtet. Dies ermöglicht an einigen Stellen eine einfacher zu berechnende Gütefunktion. So ist mit $J_{\text {Traflnfl }}$ auch die individuelle Prädiktion des rückwärtigen Verkehrs für jeden einzelnen Trajektorienkandidaten vergleichsweise einfach realisierbar. Bei der Spline-Planung wurde Wert auf eine stetig differenzierbare Gütefunktion gelegt, bei der der Gradient analytisch berechnet wird. So kann die Optimierung der 30 Parameter unter Ausnutzung der Gradienteninformation stattfinden.

Die geschätzte Beschleunigung der Umfeldfahrzeuge wird bei der Spline-Planung im Rahmen der Umfeldprädiktion genutzt. Diese Form der Prädiktion lässt sich nicht direkt auf die DreiSegment-Planung übertragen, da dadurch die Form der Wechsellücke komplexer würde und gegebenenfalls auch zwei oder mehr Wechsellücken auftreten könnten.

Bei der Drei-Segment-Planung wird die Form der Trajektorien maßgeblich durch die Generierung (Abschnitt 4.3) festgelegt. Die Gütefunktion dient dazu, die maximal drei 
verbliebenen Parameter $\left(\bar{v}_{1}, \bar{v}_{2}, v_{\mathrm{e}}\right)$ bestmöglich einzustellen. Zusätzlich lässt sich daran ablesen, ob ein Fahrstreifenwechsel durchführbar ist. Bei der Spline-Planung wird die Form der Trajektorie dagegen primär durch die Wahl der Gütefunktion beeinflusst.

Bei der Spline-Planung hat die Trajektorie eine feste zeitliche Länge, während diese bei der DreiSegment-Planung variabel ist. So bleibt der Endpunkt der Trajektorie bei der Drei-SegmentPlanung weitestgehend am selben Punkt. Dagegen verschiebt sich dieser bei der Spline-Planung im Laufe der Fahrt immer weiter in die Zukunft.

\subsubsection{Vergleich der Trajektorien}

Zum Vergleich der entstehenden Trajektorien wird wieder ein Fahrstreifenwechsel mit vier Umfeldfahrzeugen betrachtet (Abbildung 6.1). Weitere Vergleichsszenarien sind, aus Gründen der Übersichtlichkeit, in Anhang C dargestellt.

Bei den dargestellten Trajektorien handelt es sich um das Ergebnis eines einzelnen Planungsschrittes. Zur Vergleichbarkeit werden Umfeldfahrzeuge mit konstanter Geschwindigkeit betrachtet. Durch die zyklische Neuplanung kann der ausgegebene Sollgrößenverlauf von der ursprünglich geplanten Trajektorie abweichen. Dies ist insbesondere dann der Fall, wenn sich das Umfeld, z. B. als Reaktion auf den Fahrstreifenwechsel, anders verhält, als dies prädiziert wurde. Auch die Tatsache, dass sich der Planungshorizont der Spline-Planung verschiebt und so der Endpunkt immer weiter in der Zukunft liegt, führt zu einer Veränderung der Trajektorie im Laufe der Zeit. Am besten lassen sich diese Effekte im Rahmen von Fahrtests berücksichtigen. Die Ergebnisse dazu befinden sich an den Enden der beiden Trajektorienplanungskapitel in den Abschnitten 4.7 und 5.9. Zusätzlich werden in Anhang C auch einige Sollzustandsverläufe verglichen, die sich bei Umfeldfahrzeugen mit konstanter Geschwindigkeit ergeben.

Beim Vergleich der Trajektorien der beiden Planungen in Abbildung 6.1 ist erkennbar, dass sich die Längspositionsverläufe sehr ähneln. Je nach Szenario ist dies jedoch nicht immer der Fall. Die Form der Quertrajektorie ist ebenfalls sehr ähnlich. Durch Berücksichtigung der Relativgeschwindigkeit startet die Querbewegung der Spline-Trajektorie allerdings etwas früher. Auch gut zu erkennen ist die Tatsache, dass der Längsbeschleunigungsverlauf der SplinePlanung die gepunktete Maximalbeschleunigung sehr gut ausnutzen kann. Die Drei-SegmentPlanung ist in dieser Hinsicht eingeschränkter.

Noch deutlicher wird dies in Abbildung 6.2. Durch ein rückwärtiges Fahrzeug auf dem Zielfahrstreifen wird die Trajektorie, bedingt durch die Kollisionsvermeidungsterme, zum Beschleunigen gedrängt. Die Beschleunigung der Drei-Segment-Trajektorie überschreitet dabei den Grenzwert, da keine andere taugliche Trajektorie möglich ist. Dies wird erkannt und ein Wechsel in diese Lücke ist daher nicht möglich. Das System würde auf eine Lücke weiter hinten warten. Die Spline-Planung kann hier die Beschleunigung besser ausnutzen, so dass ein Wechsel in die vordere Lücke noch möglich ist. 


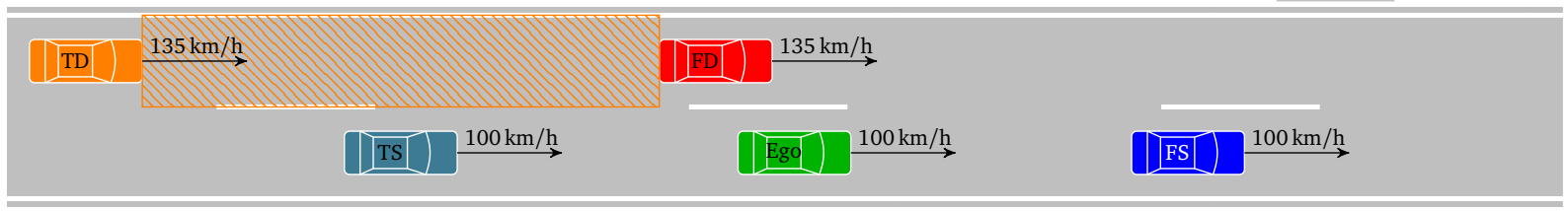
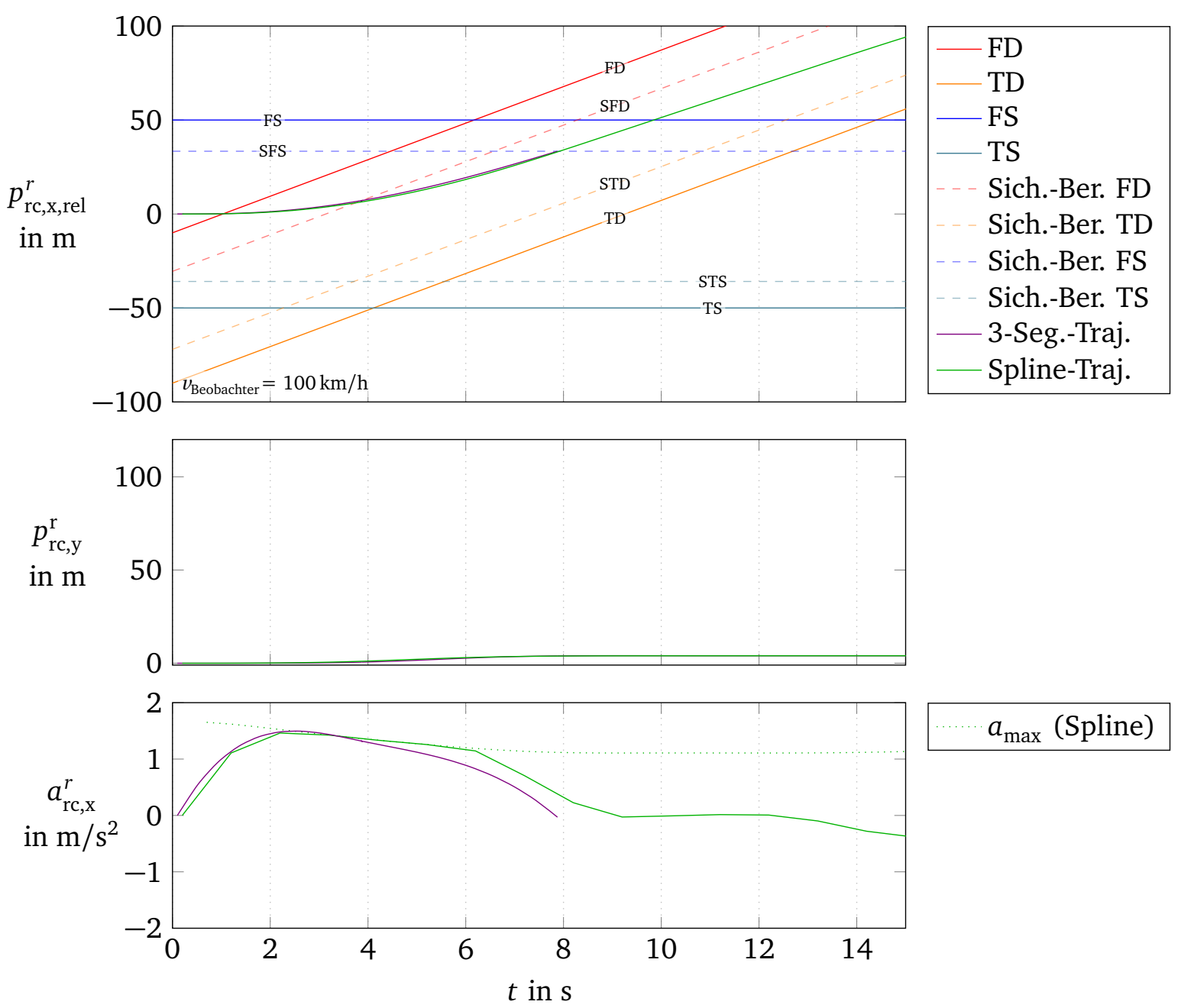

Abbildung 6.1: Vergleich der beiden sich ergebenden Trajektorien in einem Fahrstreifenwechselszenario mit vier Umfeldfahrzeugen 



$a_{\max }$ (3-Seg.)

$a_{\max }$ (Spline)

Abbildung 6.2: Situation bei der die Spline-Planung, im Gegensatz zur Drei-Segment-Planung, noch eine gültige Trajektorie planen kann. Die Drei-Segment-Trajektorie überschreitet die gestrichelte Maximalbeschleunigung stark und würde nicht ausgewählt.

\subsubsection{Vergleich der Konvergenz der beiden Planungsverfahren}

Um die Abhängigkeit der Trajektorienqualität von der Anzahl der Funktionsauswertungen durch den Optimierer darzustellen, wird für beide Planungsmethoden die jeweils beste Trajektorie nach der angegebenen Anzahl an Funktionsauswertungen dargestellt. Als Startlösung wurde für beide Planungen eine Weiterfahrt geradeaus mit konstanter Geschwindigkeit gewählt ${ }^{1}$ (Abbildung 6.3).

Wie erkennbar ist, ist die Drei-Segment-Planung in der Lage, schon ab der ersten Funktionsauswertung eine gute Quertrajektorie zu planen. Dies liegt daran, dass der nichtlineare Optimierer

$\overline{\text { Drei-Segment-Planung: } v_{1}=v_{2}=v_{\mathrm{e}}=} v_{\text {ego,ist }}$. Spline-Planung $\underline{\mathbf{j}}=\underline{\mathbf{0}}$. 


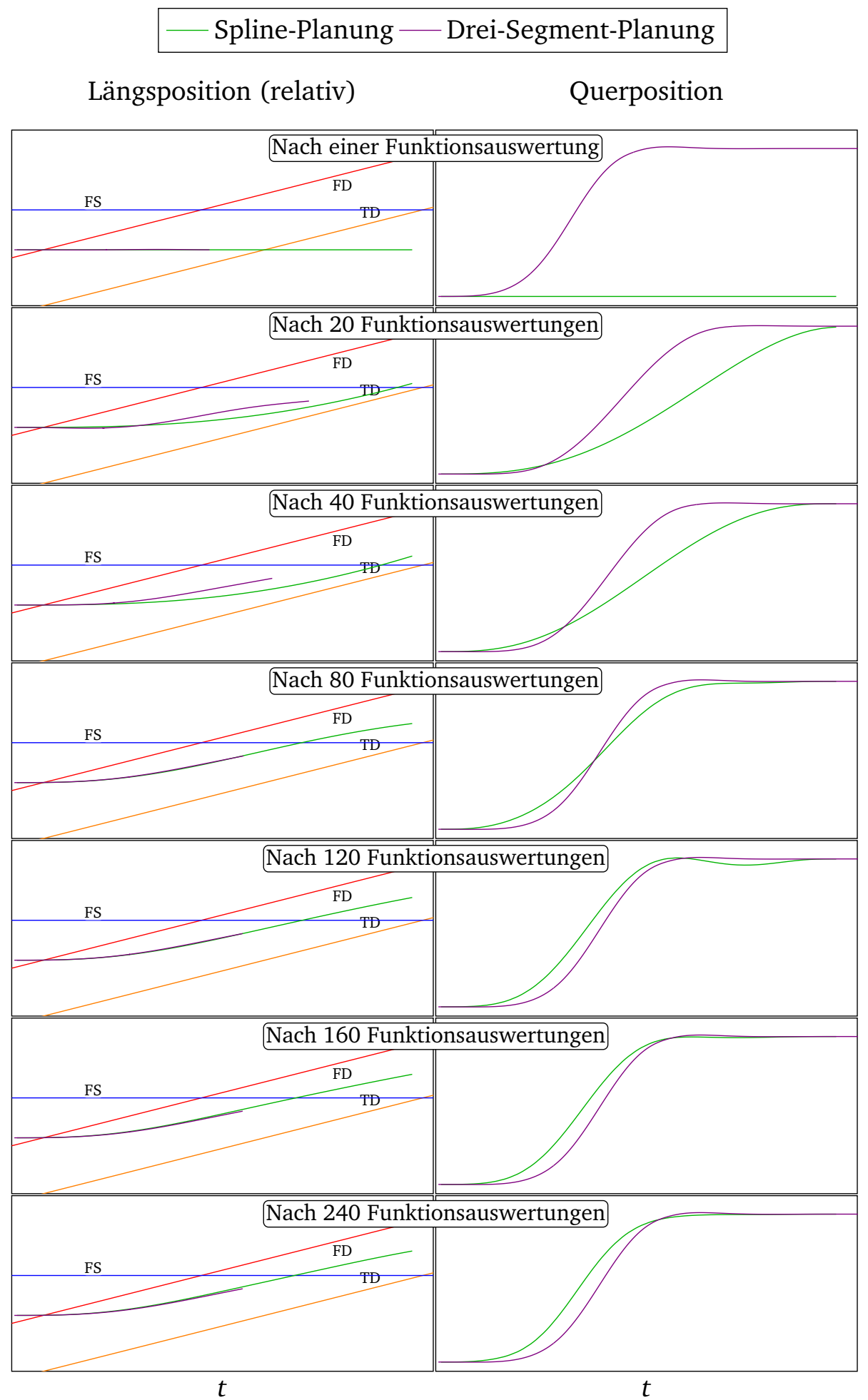

Abbildung 6.3: Entwicklung der Längstrajektorie und Quertrajektorie für Drei-Segment-Planung und Spline-Planung im Laufe eines Optimierungsdurchlaufes 
nur für die Wahl der geeigneten Längstrajektorie benötigt wird. Die zur Längstrajektorie passende Quertrajektorie wird in jedem Schritt direkt berechnet.

Wie in Abbildung 6.3 und Abbildung 6.6 zu erkennen ist, wird bei der Spline-Planung zunächst innerhalb der ersten ca. 60 Funktionsauswertungen eine gültige, kollisionsfreie Trajektorie bestimmt. Anschließend finden noch kleinere Anpassungen an der Trajektorie zur Optimierung des Komforts statt.

In Abbildung 6.3 und Abbildung 6.4 sieht man, dass die Drei-Segment-Planung weniger Funktionsauswertungen benötigt, um eine gute Trajektorie zu bestimmen. So ist die DreiSegment-Planung hier nach etwa 60 Funktionsauswertungen, die Spline-Planung nach etwa 150 Funktionsauswertungen konvergiert. In Abbildung 6.5 und Abbildung 6.6 wird dargestellt, wie sich die Güteterme im Verlauf des Optimierungsdurchlaufes verändern.

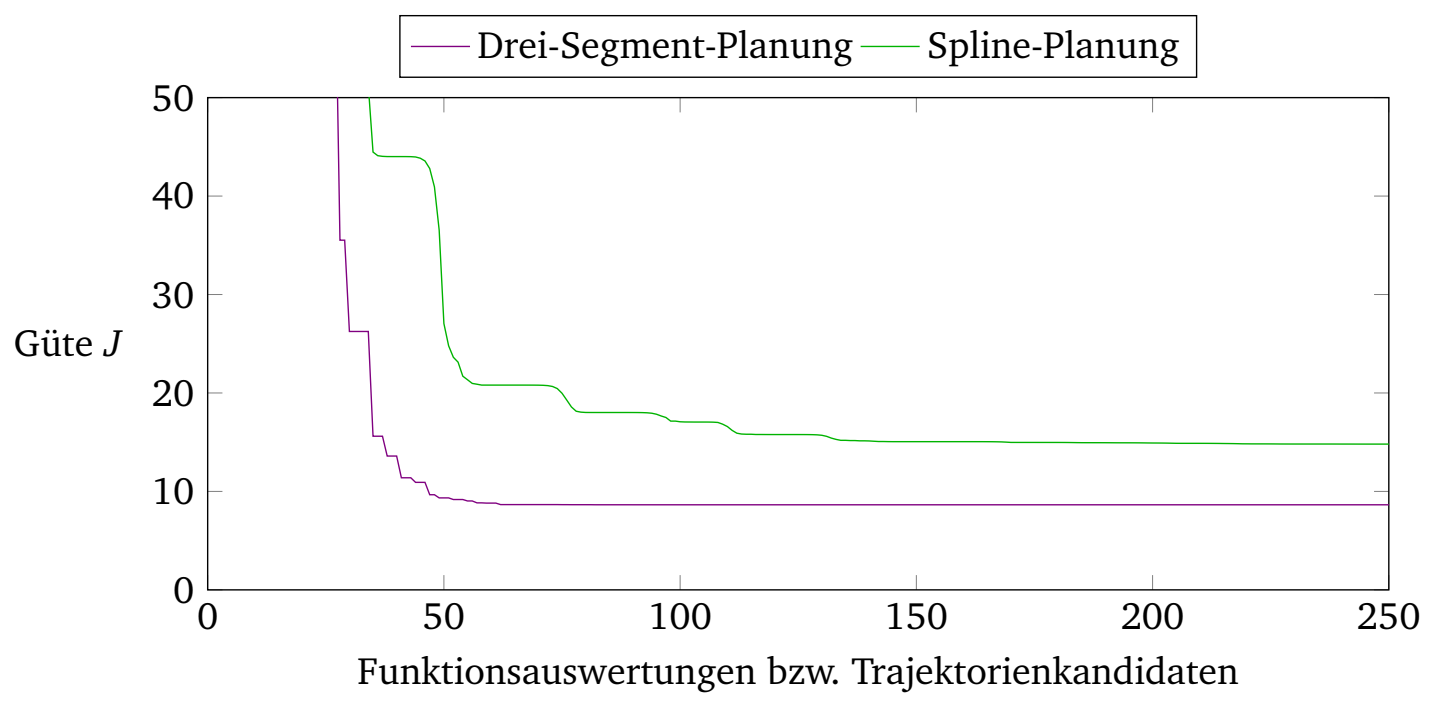

Abbildung 6.4: Vergleich der Trajektoriengüten von Drei-Segment- und Spline-Planung in Abhängigkeit der Anzahl an Funktionsauswertungen

Durch die Wahl einer besseren Startlösung lässt sich die Anzahl der Funktionsauswertungen noch weiter reduzieren. Dies gilt insbesondere für die Spline-Planung. 


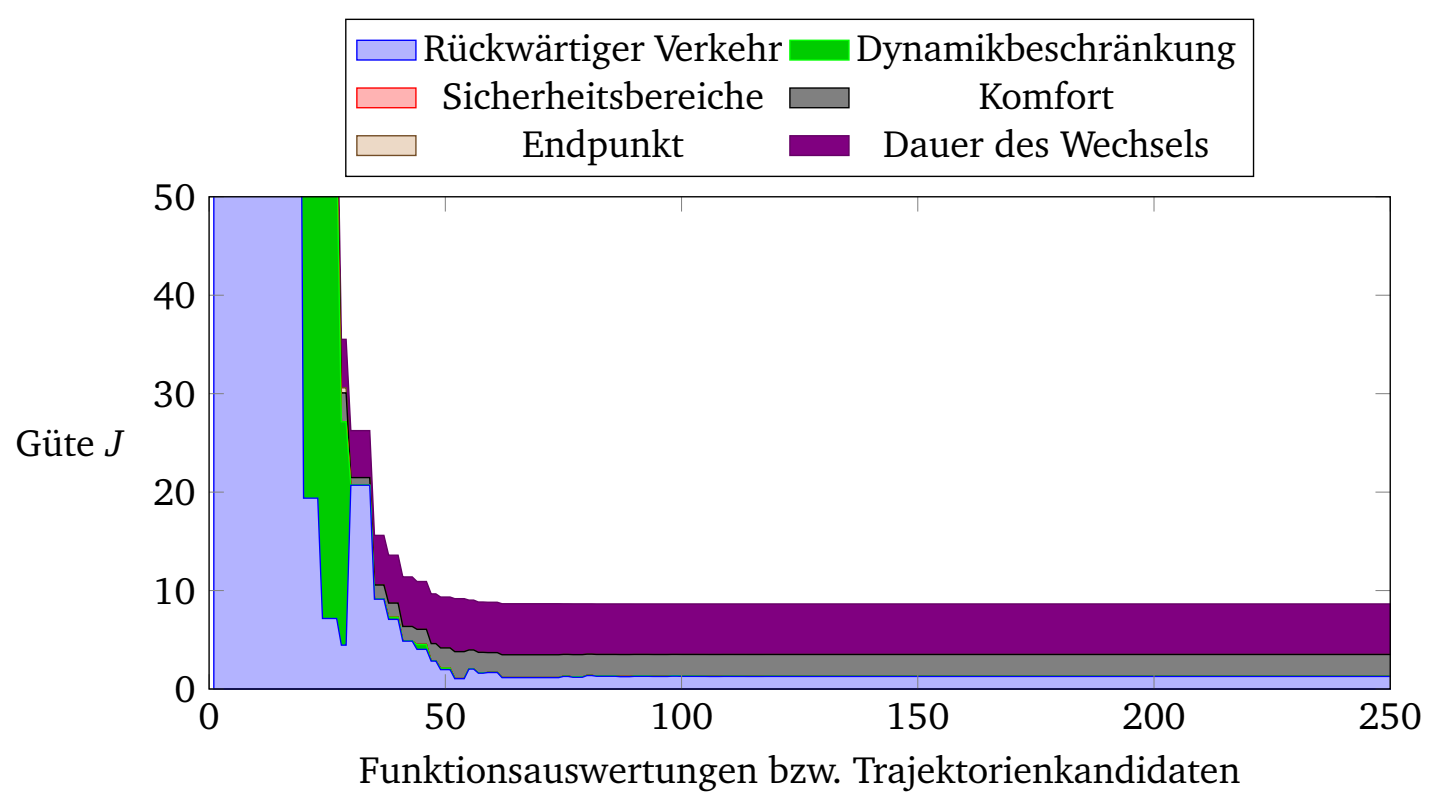

Abbildung 6.5: Entwicklung der Güteterme der Drei-Segment-Planung im Laufe des Optimierungsdurchlaufes

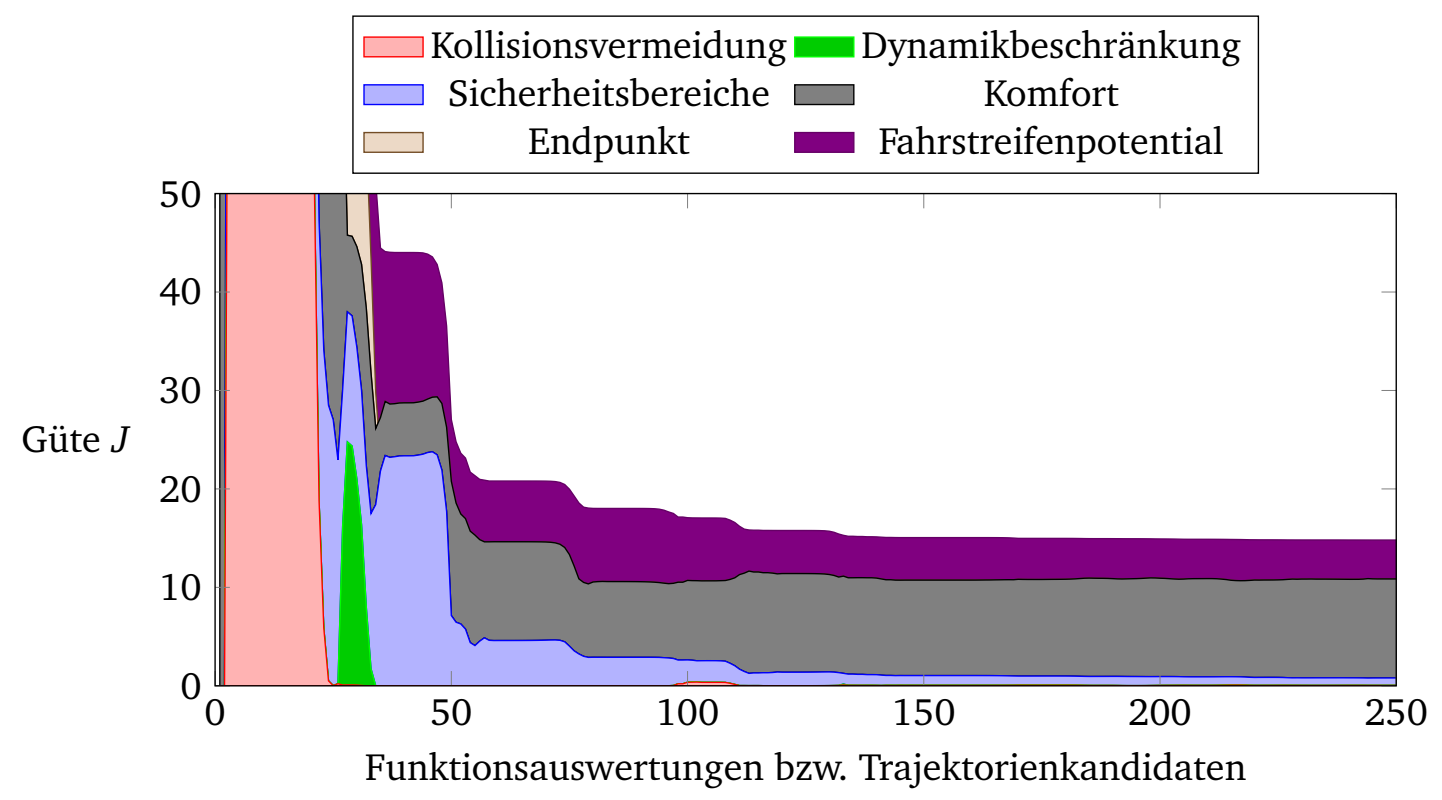

Abbildung 6.6: Entwicklung der Güteterme der Spline-Planung im Laufe des Optimierungsdurchlaufes 
Zum Vergleich der benötigten Rechenzeiten wurden die Ausführungszeiten beider Trajektorienplanungen auf dem Messrechner (CPU: Intel i7-3740QM) verglichen (Tabelle 6.1). Für die DreiSegment-Planung wurde wieder ausschließlich das Worst-Case-Szenario mit der Planung von drei Segmenten betrachtet. Die Rechenzeit für Trajektorien mit zwei oder nur einem Segment ist wesentlich geringer.

Tabelle 6.1: Vergleich der benötigten Rechenzeiten auf dem Messrechner

\begin{tabular}{lrrc} 
& Spline & Drei-Seg. & Einheit \\
\hline Dauer pro Trajektorienkandidat & 63 & 31 & $\mu \mathrm{s}$ \\
Kandidaten pro Trajektorie & 150 & 100 & \\
Grunddauer pro Trajektorie & 3 & 1,5 & $\mathrm{~ms}$ \\
Dauer pro Trajektorie & 12,5 & 4,6 & $\mathrm{~ms}$
\end{tabular}

Die Gesamtdauer für eine Trajektorie setzt sich zusammen aus der Dauer für einen Trajektorienkandidaten multipliziert mit der Anzahl der Kandidaten plus einer Grunddauer. Die Spline-Planung benötigt aktuell etwa dreimal so viel Rechenzeit wie die Drei-Segment-Planung. Dieser Vergleich ist nicht besonders genau, da der Quellcode aus MATLAB heraus erzeugt wurde und somit seine Effizienz nicht bekannt ist. Es wird davon ausgegangen, dass die Abweichung zu den in Abschnitt 4.8 beschriebenen theoretischen Berechnungszeiten auf die geringe Effizienz des erzeugten Quellcodes zurückzuführen ist.

Der größte Unsicherheitsfaktor liegt allerdings in der Anzahl der benötigten Funktionsauswertungen bzw. Trajektorienkandidaten. Wird das Umfeld sicher erfasst, muss die Trajektorie in den folgenden Schritten nur etwas angepasst werden. Tauchen jedoch Fahrzeuge plötzlich auf, so muss die Trajektorie unter Umständen stark verändert werden, was mehr Funktionsauswertungen benötigt.

\subsubsection{Stärken und Schwächen der beiden Planungsmethoden}

Im Vergleich der beiden Trajektorienplanungsmethoden hebt sich die Drei-Segment-Planung insbesondere durch die geringe benötigte Rechenzeit hervor. Die Optimierung ist sehr robust und liefert auch mit einer geringen Anzahl an Funktionsauswertungen gute Trajektorien. Nachteilig sind die notwendigen Fallunterscheidungen (Anhang A.6). Diese sind erforderlich, um die Anzahl der zu berechnenden Segmente zu bestimmen und orientieren sich an einem normalen Fahrstreifenwechsel. Bei besonderen Situationen, wie schwierigen Abbrüchen und ähnlichem, kann es sein, dass diese Fallunterscheidungen nicht passen und keine Trajektorie planbar ist oder diese nicht zielführend ist. 
Die Spline-Planung ist in der Lage, die Fahrzeugmöglichkeiten besser auszunutzen als die DreiSegment-Planung. Durch ihre größere Anzahl an Freiheitsgraden kann sie besser auf das Umfeld reagieren. Sie benötigt zwar mehr Rechenzeit, ist aber in ihrer Trajektorie wesentlich flexibler. Es ist hier vergleichsweise einfach, Detailanpassungen durch Änderung der Gütefunktion vorzunehmen. So lässt sich die Spline-Planung auch auf Situationen mit langsam schmaler werdenden Fahrstreifen erweitern. Das Gleiche gilt, sofern die Umfelderfassung gut genug ist, auch für die Nutzung einer prädizierten Querposition der Umfeldfahrzeuge. Dies ist bei der DreiSegment-Planung nur äußerst schwer berücksichtigbar. Die Spline-Planung lässt sich auch gut für eine reine Längsplanung im Sinne eines ACCs verwenden, so dass keine Umschaltvorgänge vorhanden sind. In Abschnitt 6.3 werden zudem einige Ansätze zur Reduktion der Rechenzeit aufgezeigt.

\subsection{Erweiterungsmöglichkeiten}

Bei den Fahrtests mit der Drei-Segment-Planung fiel auf, dass die Verwendung des IntelligentDriver-Models (IDM) als ACC in manchen Situationen nicht besonders komfortabel ist. Es reagiert auf nah vorausfahrende Fahrzeuge sehr konservativ und bremst stark. Hier wäre es wünschenswert, eine vereinfachte echte ACC-Funktion zur Festlegung des Endpunktes zu verwenden und am Ende der Längstrajektorie an das echte ACC zu übergeben. Dabei ist zu beachten, dass die ACC-Funktion für jeden Trajektorienkandidaten ausgewertet wird und daher nicht zu rechenzeitintensiv sein darf. Alternativ könnte eine verbesserte Version des IDM verwendet werden, wie sie in [Treiber, 2013, S. 198-201] beschrieben ist.

Die aktuellen Fallunterscheidungen der Drei-Segment-Planung sind für einen normalen Fahrstreifenwechsel ausgelegt. Für einen Serieneinsatz ist noch zu klären, inwieweit sie auch für den Abbruch in kritischen Szenarien (Anhang D) geeignet sind. Gegebenenfalls sind einige Spezialfälle separat zu behandeln.

Die Tatsache, dass der rückwärtige Verkehr auf dem Ego-Fahrstreifen beim Start des Wechsels komplett ausgeblendet wird, führt gelegentlich zu einem unnatürlichen Verhalten. Da dessen notwendige Verzögerung nicht in das Gütekriterium eingeht, kann es beispielsweise bei einem Wechsel auf den rechten Fahrstreifen dazu kommen, dass die Planung sich für die hintere der zulässigen Lücken entscheidet und so den rückwärtigen Verkehr auf dem Start-Fahrstreifen ausbremst, um in die Lücke wechseln zu können. Diese Situation ist in Abbildung 6.7 dargestellt. Hier wäre es häufig wünschenswert, in eine Lücke weiter vorne zu wechseln. Dazu wäre es möglich, entweder das rückwärtige Fahrzeug auf dem eigenen Fahrstreifen im Gütekriterium gesondert zu berücksichtigen oder auf Ebene der Ziellückenauswahl (Abschnitt 2.6) einzubeziehen.

Für die Bedienung der Funktion durch den Fahrer ist es sehr wichtig, dass das Rechtsüberholen von Fahrzeugen durch die Trajektorienplanung und das ACC verhindert wird. Zwar wird die Lücke links vorne ausgeblendet, dies allein reicht jedoch zur Verhinderung des Rechtsüberholens 




$\underset{\square}{\longrightarrow} \stackrel{100 \mathrm{~km} / \mathrm{h}}{\longrightarrow} \stackrel{\mathrm{km} / \mathrm{h}}{\longrightarrow} \square$

Abbildung 6.7: Situation, in der das Auswählen der weiter vorne liegenden Lücke vorteilhafter wäre

nicht aus. Dies wird im Folgenden anhand zweier Szenarien verdeutlicht. In Abbildung 6.8 wird ein Szenario gezeigt, bei dem das eigene Fahrzeug zunächst auf dem rechten Fahrstreifen fährt. Auf dem linken Fahrstreifen fahren die Fahrzeuge mit wechselnder Geschwindigkeit. Für einen Wechsel auf den linken Fahrstreifen muss die Setzgeschwindigkeit ausreichend hoch eingestellt sein. Dabei dürfen die Fahrzeuge vor Beginn des Wechsels nicht auf der rechten Seite überholt werden.



Abbildung 6.8: Szenario, in dem es nicht zu einem Überholen der Fahrzeuge auf dem linken Fahrstreifen kommen darf

In gewissen Situationen kann es sogar notwendig sein, das Fahrzeug auf dem übernächsten Fahrstreifen zu berücksichtigen (Abbildung 6.9).

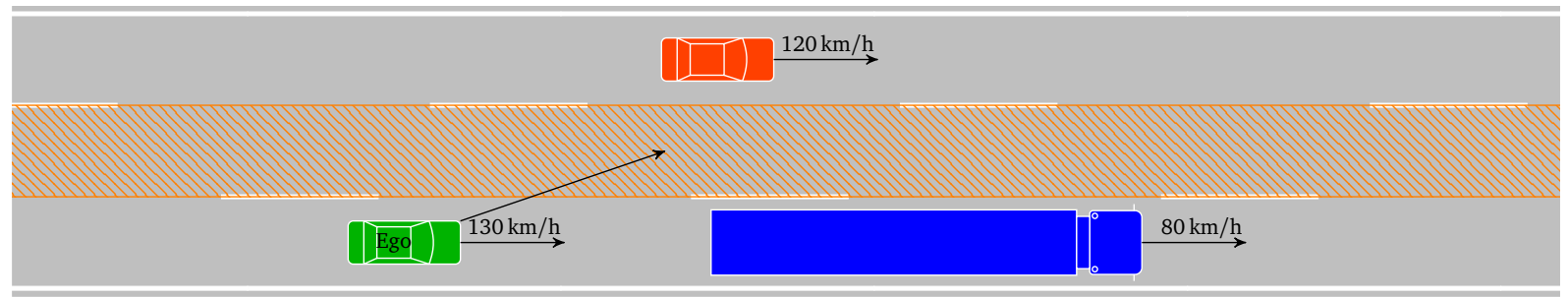

Abbildung 6.9: Kritisches Szenario, bei dem das Fahrzeug auf dem übernächsten Fahrstreifen mit in die Planung einbezogen werden muss, um ein Rechtsüberholen zu vermeiden.

Um diese Regeln in der Trajektorienplanung zu berücksichtigen, könnte bei der Drei-SegmentPlanung eine ACC-Funktion berechnet werden, die das Fahrzeug auf dem linken Fahrstreifen mit berücksichtigt. Dies wurde zum Teil auch schon umgesetzt, es wurde allerdings festgestellt, dass nicht eine normale ACC Funktion verwendet werden darf, denn dann bremst das eigene Fahrzeug immer, wenn es von einem anderen Fahrzeug überholt wird. Bei der SplinePlanung ließe sich neben dem relevanten Fahrzeug ein Potential-Berg erstellen, der von der Relativgeschwindigkeit abhängig ist. Dieser Potentialberg könnte so auslegt werden, dass er in 
kritischen Situationen (linkes Fahrzeug führt eine sehr starke Bremsung durch) durchfahren werden kann.

Die Drei-Segment-Planung ließe sich so abwandeln, dass die Zeitpunkte $t_{1}$ und $t_{2}$ sowie die Positionen $p_{1}$ und $p_{2}$ nicht aus dem Umfeld bestimmt werden, sondern auch Ergebnis der Optimierung sind. Anstatt drei Parametern sind dann fünf zu optimieren. Damit würde sie zwar die Beschleunigungsmöglichkeiten immer noch nicht so gut ausnutzen wie die SplinePlanung, könnte jedoch mehr Freiräume nutzen, und wäre bei Abbruchszenarien eventuell flexibler. Durch die fünf zu optimierenden Parameter wäre es sinnvoll, den Gradienten der Gütefunktion bezüglich der Parameter berechnen zu können, um die Rechenzeit nicht zu stark anwachsen zu lassen. Eventuell ließe sich der Gradient auch bei der aktuellen Drei-SegmentPlanung dazu nutzen, die Rechenzeit weiter zu reduzieren.

Im Laufe der Arbeit hat sich gezeigt, dass es bei der aktuellen Parametrierung der Spline-Quertrajektorie über den Ruck bei einer hohen Endpunktgewichtung zu numerisch bedingten Nebenminima kommen kann. Aus diesem Grund wird aktuell die Gewichtung des Endpunktes niedriger gewählt, wenn sich dieser bei der Start-Lösung aus dem letzten Optimierungsdurchlauf in der Nähe der Ziel-Fahrstreifenmitte befindet. Besser wäre es jedoch, die Parametrierung der Trajektorie so zu wählen, dass sie weniger anfällig in Bezug auf eine hohe Endpunktgewichtung ist, oder den Endpunkt als Nebenbedingung fest vorzugeben. In Anhang B.2 werden einige Parametrierungsmöglichkeiten beschrieben. Bei einer Parametrierung über Positionen ließe sich der Endpunkt leicht fest vorgeben und so die Anzahl der Optimierungsvariablen noch reduzieren.

Durch die große Anzahl an Freiheitsgraden bei der Spline-Planung steigt das Risiko für untaugliche Trajektorien durch Nebenminima und unvorhergesehene Situationen. Daher sollten die Trajektorien zukünftig noch auf Plausibilität geprüft werden, bevor sie auf das Fahrzeug geschaltet werden.

Aktuell wird die Lösung aus dem letzten Zeitschritt nur insofern als Startwert übernommen, als dass der Parametervektor gleich bleibt. Für die Drei-Segment-Planung passt dies relativ gut. Bei der Spline-Planung führt dies, aufgrund der neuen Startwerte und der Parametrierung über den Ruck, zu einer Trajektorie, die von der letzten Lösung abweicht. Hier wäre eine präzisere Übernahme des Positionsverlaufs der letzten Trajektorie sinnvoll.

Falls für eine Lücke noch keine Trajektorie berechnet wurde, wird als Startwert für die Optimierung aktuell bei der Drei-Segment-Planung die Weiterfahrt mit konstanter Geschwindigkeit und bei der Spline-Planung die Weiterfahrt mit konstanter Beschleunigung in Längs- und Querrichtung verwendet. An dieser Stelle ließe sich eine bessere Startlösung für die Optimierung verwenden. Hierzu muss berücksichtigt werden, wo sich der Zielfahrstreifen relativ zum Fahrzeug befindet, um eine grob passende Fahrstreifenwechseltrajektorie als Startwert verwenden zu können. 
Aktuell wird bei der Spline-Planung der rückwärtige Verkehr auf dem Zielfahrstreifen nur einmal vor dem Start des Optimierungsdurchlaufes prädiziert. Es wäre denkbar, hier ein ähnliches Verfahren wie bei der Drei-Segment-Planung anzuwenden, bei dem für jeden Trajektorienkandidaten eine neue Prädiktion durchgeführt wird. Für die Spline-Planung ist die Gradientenberechnung für diese Berücksichtigung aber relativ aufwändig und vermutlich rechenzeitintensiv. Ob sich dieser Aufwand lohnt, müsste in der Praxis untersucht werden.

Die Bewertung der Abtastpunkte der Spline-Trajektorie in Bezug auf die Umfeldfahrzeuge basiert primär auf den Positionen der Fahrzeuge. Es wäre prinzipiell denkbar, zusätzlich die eigene Fahrtrichtung, sowie die Geschwindigkeitsdifferenzen zu den Umfeldfahrzeugen stärker im Gütekriterium zu berücksichtigen. Dadurch ließe sich eine Bewertung der einzelnen Zwischenzustände der Trajektorie in Bezug auf deren Sicherheit realisieren. Dies könnte dazu beitragen, dass ein Abbruch des Fahrstreifenwechsels aus möglichst jedem Zwischenzustand der Trajektorie möglich ist. Obwohl es aktuell keine Hinweise auf die Notwendigkeit dafür gibt, ließe sich so die Sicherheit des Systems weiter erhöhen.

Bei der Spline-Planung wurde die Segmentlänge $T=1 \mathrm{~s}$ größer gewählt als die Neuplanungszeit $T_{\text {Plan }}=0,2 \mathrm{~s}$. Dadurch kommt es zu dem Effekt, dass sich die ursprünglich geplante Trajektorie bei der Neuplanung nicht exakt reproduzieren lässt. Dies führt zu einem gewissen Verschleifungseffekt der Beschleunigung, wie er bei einigen Sollgrößenverlaufen in Anhang C erkennbar ist. Um dies zu vermeiden, wäre es denkbar, in den Zeitschritten, die nicht in das $1 \mathrm{~s}$ Raster passen, ein entsprechend verkürztes erstes Segment zu verwenden.

Um die für die Spline-Planung benötigte Rechenzeit zu reduzieren, gibt es unterschiedliche Möglichkeiten. So könnte die Trajektorienlänge noch etwas reduziert werden. Es ist davon auszugehen, dass die meisten Fahrstreifenwechsel auch noch bei einer Trajektorienlänge von 10 bis $12 \mathrm{~s}$ durchgeführt werden können. Des Weiteren wäre es denkbar, bei der Spline-Planung Segmente unterschiedlicher Länge zu verwenden. So könnten die zeitlich weiter entfernten Segmente länger ausfallen. Der Bereich wird damit nur grob geplant. Wenn sich das Fahrzeug der Situation nähert, wird dann die Detailplanung durchgeführt. Beide Ansätze reduzieren die Anzahl der Segmente und somit auch die Anzahl der Optimierungsvariablen.

Sofern die Umfelderfassung eine ausreichende Genauigkeit aufweist, wäre es bei der SplinePlanung sinnvoll zumindest für die Kollisionsvermeidung nicht von einer vollständigen Belegung des Fahrstreifens auszugehen. Anstatt dessen könnte, für eine begrenzte Anzahl an Umfeldfahrzeugen, direkt die Objektposition in das Potential eingehen. Sofern die Kamera in der Lage ist, eine Verengung des Fahrstreifens zu erkennen, könnte dies ebenfalls berücksichtigt werden. 


\subsection{Empfehlung}

Insgesamt ist somit davon auszugehen, dass es vorteilhafter ist, die Spline-Planung weiter zu verfolgen. Die Drei-Segment-Planung ist nur für eine Basisvariante in Form eines durch die kombinierte Längs- und Querplanung verbesserten Fahrstreifenwechselassistenten sinnvoll. Die Spline-Planung lässt sich wesentlich einfacher erweitern und ermöglicht höhere Automatisierungsgrade. Es wird davon ausgegangen, dass sich deren Rechenzeit durch die in Abschnitt 6.3 genannten Möglichkeiten noch weiter reduzieren lässt. 


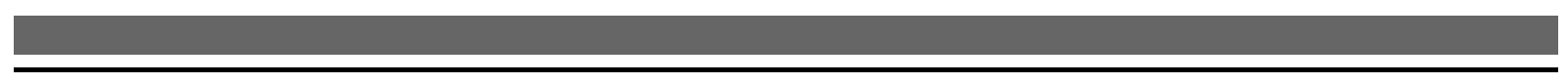




\section{Zusammenfassung \& Ausblick}

\subsection{Zusammenfassung}

Beschrieben wurden zwei Trajektorienplanungsmethoden für Fahrstreifenwechsel auf der Autobahn mit kombinierter Längs- und Querplanung. Es werden Trajektorien für unterschiedliche Lücken geplant und auf Wunsch des Fahrers die am besten passende abgefahren. Die Trajektorienplanung gibt dabei einen Verlauf für die Sollposition vor, unterlagerte Regelkreise sorgen dafür, dass das Fahrzeug diesem folgt. Beide Ansätze erlauben es, Trajektorien zu planen, bei der die Querbewegung erst zu einem zukünftigen Zeitpunkt beginnt. Dadurch kann beispielsweise schon sehr frühzeitig beschleunigt werden. Weiterhin sind beide Trajektorienplanungen in der Lage, einen Fahrstreifenwechsel abzubrechen, sofern die Situation dies erfordert. Das System wurde für den Bereich des teilautomatisierten Fahrens (SAE Level 2) ausgelegt . Eine Verwendung für das hochautomatisierte Fahren (SAE Level 3) wäre denkbar und zu prüfen.

Bei der Drei-Segment-Planung (Kapitel 4) besteht die Trajektorie aus bis zu drei Segmenten variabler zeitlicher Länge (Anfahrt, Fahrstreifenwechsel, Weiterfahrt). Für die ersten beiden Segmente kommen dabei Polynome fünften Grades zum Einsatz. Nach dem zweiten Segment wird die Längsführung durch das ACC übernommen und der verbleibende Querversatz abgebaut. Die Trajektorie der Spline-Planung (Kapitel 5) besteht aus 15 Segmenten konstanter zeitlicher Länge, die jeweils durch ein Polynom dritten Grades beschrieben werden, sodass, wie bei der Drei-Segment-Planung, ein stetiger Verlauf der Beschleunigung erzielt wird. Beide Planungen berücksichtigen die beschränkte Fahrzeugdynamik sowie die Umfeldobjekte. Bei beiden Planungsmethoden ist die Trajektorie Ergebnis einer nichtlinearen Optimierung. Die 30 Optimierungsvariablen der Spline-Planung werden hierbei unter Ausnutzung des Gradienten der Gütefunktion optimiert. Da bei der Drei-Segment-Planung nur maximal drei Optimierungsvariablen zu optimieren sind, geschieht dies ohne Nutzung des Gradienten.

Um die Praxistauglichkeit der Verfahren zu zeigen, wurden beide Trajektorienplanungen in einem Versuchsfahrzeug implementiert. Nachdem das System simulativ in verschiedenen Szenarien geprüft wurde, wurden die Ergebnisse mit echten Fahrzeugen auf einem Testgelände verifiziert. Anschließend fanden Fahrtests im realen Straßenverkehr auf der Autobahn statt. Dabei konnte gezeigt werden, dass das System in der Lage ist, sichere Fahrstreifenwechsel auch in dichtem Verkehr durchzuführen. Die Fahrstreifenwechsel wurden von den Fahrern als komfortabel und sehr angenehm beschrieben. Insgesamt wurden mehr als $1300 \mathrm{~km}$ im Rahmen von Testfahrten zurückgelegt (Kapitel 6). 
Eine Abschätzung der Rechenzeit hat ergeben, dass beide Planungsmethoden auf einem herkömmlichen Steuergerät lauffähig sein sollten. Die Drei-Segment-Planung hat den Vorteil, dass die sich ergebenden Trajektorien relativ gut vorhersehbar sind und sie weniger Rechenzeit benötigt. Die bei der Planung notwendigen Fallunterscheidungen sind aber auf einen normalen Fahrstreifenwechsel ausgelegt und für unvorhergesehene Situationen nur bedingt geeignet. Die Spline-Planung ist rechenzeitintensiver, aber auch deutlich flexibler. So kann sie einfacher auf sich verengende Fahrstreifen sowie fahrstreifenwechselnde Fahrzeuge angepasst werden. Insgesamt sind damit höhere Automatisierungsgrade möglich. Daher wird die Weiterverfolgung der Spline-Planung empfohlen.

Die Grundzüge der Drei-Segment-Planung wurden in einem Artikel in der „ATZ - Automobiltechnische Zeitschrift" veröffentlicht [Hansen, 2016b]. Die der Spline-Planung wurden auf der Konferenz AUTOREG 2017 in Berlin vorgestellt [Hansen, 2017].

\subsection{Ausblick}

Neben den in Abschnitt 6.3 beschriebenen Erweiterungsmöglichkeiten kann das System, unter Annahme einer ausreichenden Sensorreichweite, auch für zusätzliche Funktionen verwendet werden.

Da das System kontinuierlich Trajektorien für unterschiedliche Fahrstreifen plant, könnte es die Entscheidung zu einem Fahrstreifenwechsel auch selbstständig vornehmen. Auf diese Weise würden vollautomatische Überholvorgänge auf der Autobahn durchgeführt. Auch für das automatisierte Auffahren auf die Autobahn wären die Trajektorienplanungen im Rahmen eines Einfädelassistenten prinzipiell geeignet. Die kontinuierlich stattfindende Trajektorienplanung erlaubt weiterhin die Bewertung der aktuellen Situation auch bei einer manuellen Fahrt. Dies könnte genutzt werden, um den Fahrer in kritischen Situationen frühzeitig zu warnen.

Bei der Spline-Planung wäre es denkbar, bei entsprechender Sensorik, auch eine teilweise Belegung der Fahrstreifen durch die Umfeldfahrzeuge zu berücksichtigen. Im Rahmen eines integrierten Ansatzes könnte die Längs- und Querführung dauerhaft von der Spline-Planung übernommen werden. Dadurch könnte, bei Verwendung entsprechender Güteterme, auch eine energieoptimale Fahrweise und somit eine Verbrauchs- und Emissionsminimierung erzielt werden. Denkbar wäre als Güteterm beispielsweise die durch Bremsen in Wärme umgewandelte Energie.

Bis zu einer gewissen Dynamik wäre auch ein Ausweichen vor Hindernissen möglich. Dies könnte zum Beispiel ein liegengebliebenes Fahrzeug sein oder eines, das seinen Fahrstreifen aufgrund von Unachtsamkeit langsam verlässt. In Querrichtung könnte anstatt der Integratorkette auch ein anderes lineares System, wie z. B. das lineare Einspurmodell verwendet werden. Zusammen mit einer kürzeren Trajektorienlänge, könnten so auch Trajektorien in dynamischeren Situationen geplant werden. 
Es ist zu untersuchen, wie sich das System auf höhere Automatisierungsgrade bzw. SAE-Level erweitern lässt. Interessant wäre hierbei die Untersuchung der von der Straßenverkehrsordnung geforderten Rücksicht auf andere Verkehrsteilnehmer. Hierfür könnte eine detaillierte Prädiktion des Umfelds, insbesondere in Querrichtung, hilfreich sein. Die Entscheidungsmöglichkeiten der anderen Fahrer könnten in einem probabilistischen Ansatz berücksichtigt werden. Hierzu gehört insbesondere die Entscheidung, ob ein Fahrer den Fahrstreifen wechselt oder nicht.

Im Rahmen der Versuchsfahrten hat sich deutlich gezeigt, dass das eigene Verhalten bzw. die eigene Trajektorie das Umfeld beeinflusst. Teilweise wurde dies auch schon in einigen Gütetermen berücksichtigt. Im Hinblick auf das entstehende Gesamtsystem könnte erforscht werden, wie sich diese Abhängigkeit der Trajektorien untereinander modellieren lässt. Gegebenenfalls ist es auch sinnvoll, dass das eigene Fahrzeug Trajektorien für andere Fahrzeuge plant, um deren Situation besser einschätzen zu können.

Neben der Autobahnfahrt ist es auch denkbar, Trajektorien auf Basis des Spline-Ansatzes für urbane Verkehrssituationen zu planen. Weiterhin könnte der Ansatz auch in anderen Bereichen wie z. B. der allgemeinen Robotik oder für unbemannte Flugobjekte genutzt werden.

Abgesehen von den beschriebenen Erweiterungsmöglichkeiten und alternativen Einsatzmöglichkeiten könnten weitere Schritte in Hinblick auf einen Serieneinsatz des Systems unternommen werden. Für die dafür notwendige Absicherung wären weitere Sicherheits- und Plausibilitätsüberprüfungen sinnvoll. Abschließend wäre das System, unter Beachtung der dafür relevanten Normen, auf dem Steuergerät zu implementieren und zu parametrieren sowie eine Erprobung durchzuführen. 


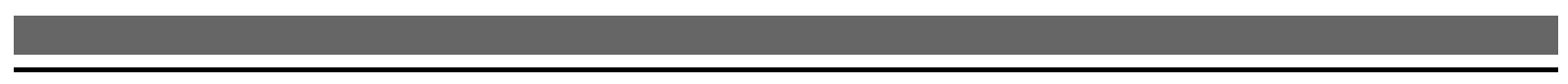




\section{A Detailbetrachtungen der Drei-Segment-Planung}

Im ersten Teil des Kapitels befinden sich die Details zur Generierung der Trajektorien. Anschließend sind unterschiedliche Parametrierungsmöglichkeiten der Quertrajektorie dargestellt. In Abschnitt A.4 wird dann die Bestimmung der maximalen Fahrstreifenwechseldauer beschrieben. Abschließend werden die auftretenden Fallunterscheidungen sowie die maximal zulässige Abtastzeit betrachtet.

\section{A.1 Generierung der Drei-Segment-Trajektorie}

Sowohl die Längs- als auch die Quertrajektorie bestehen in den ersten beiden Segmenten aus Polynomen 5. Grades. Bei beiden wird ein stetiger Verlauf der Position $p$, der Geschwindigkeit $v$ und der Beschleunigung a gefordert. Die grundlegenden Berechnungen sowie das Einsetzen der Nebenbedingungen sind daher für Längs- und Querrichtung gleich und werden im Folgenden gezeigt.

Ziel der Berechnungen ist es, die Stetigkeitsbedingungen einzusetzen, um später ein Optimierungsproblem mit möglichst wenig Optimierungsvariablen zu erhalten. Im Gegensatz zur Verwendung von Lagrange-Multiplikatoren kann so Rechenzeit eingespart werden.

Wie erwähnt, besteht die Trajektorie aus zwei Polynomsegmenten. Der Positionsverlauf $p(t)$ lässt sich daher mit der Basisfunktion $\underline{\underline{f}}^{\mathrm{T}}(t)=\left[\begin{array}{llllll}t^{5} & t^{4} & t^{3} & t^{2} & t & 1\end{array}\right]$ wie folgt darstellen:

$$
p(t)= \begin{cases}p_{1}(t)=\underline{\mathbf{f}}^{\mathrm{T}}(t) \cdot \underline{\mathbf{a}} & 0 \leq t<t_{1} \\ p_{2}(t)=\underline{\mathbf{f}}^{\mathrm{T}}\left(t-t_{1}\right) \cdot \underline{\mathbf{b}} & t_{1} \leq t \leq t_{2}\end{cases}
$$

Die Vektoren $\underline{\mathbf{a}}$ und $\underline{\mathbf{b}}$ stehen dabei für die Polynomkoeffizienten.

Mit dem Zustandsvektor $\underline{\underline{\mathbf{x}}}=\left[\begin{array}{llll}p & v & a & j\end{array}\right]^{\mathrm{T}}$ lassen sich auch Geschwindigkeit, Beschleunigung und Ruck kompakt in die Darstellung integrieren:

$$
\underline{\mathbf{x}}(t)= \begin{cases}\underline{\mathbf{x}}_{1}(t)=\underline{\mathbf{F}}(t) \cdot \underline{\mathbf{a}} & 0 \leq t<t_{1} \\ \underline{\mathbf{x}}_{2}(t)=\underline{\mathbf{F}}\left(t-t_{1}\right) \cdot \underline{\mathbf{b}} & t_{1} \leq t \leq t_{2}\end{cases}
$$

Die Matrix $\underline{\mathbf{F}}(t)$ enthält dabei die notwendigen Basisfunktionen:

$$
\underline{\mathbf{F}}(t)=\left[\begin{array}{c}
\underline{\mathbf{f}}^{\mathrm{T}}(t) \\
\underline{\mathbf{f}}^{\mathrm{T}^{\prime}}(t) \\
\underline{\mathbf{f}}^{\mathbf{T}^{\prime \prime}}(t) \\
\underline{\mathbf{f}}^{\mathrm{T}^{\prime \prime \prime}}(t)
\end{array}\right]=\left[\begin{array}{ccc|ccc}
t^{5} & t^{4} & t^{3} & t^{2} & t & 1 \\
5 t^{4} & 4 t^{3} & 3 t^{2} & 2 t & 1 & 0 \\
20 t^{3} & 12 t^{2} & 6 t & 2 & 0 & 0 \\
60 t^{2} & 24 t & 6 & 0 & 0 & 0
\end{array}\right]=\left[\underline{\mathbf{F}}_{53}(t) \mid \underline{\mathbf{F}}_{20}(t)\right]
$$


Ein Teil der Polynomkoeffizienten ergibt sich aus den Randbedingungen an den Anfangspunkten der Segmente, die anderen sind frei wählbar. Es ist daher sinnvoll, die Polynomkoeffizienten in einen abhängigen Teil $\underline{\mathbf{a}}_{20}, \underline{\mathbf{b}}_{20}$ und einen frei wählbaren Teil $\underline{\mathbf{a}}_{53}, \underline{\mathbf{b}}_{53}$ zu unterteilen:

$$
\begin{aligned}
& \underline{\mathbf{a}}=\left[\begin{array}{c}
a_{5} \\
a_{4} \\
a_{3} \\
\hline a_{2} \\
a_{1} \\
a_{0}
\end{array}\right]=\left[\begin{array}{l}
\underline{\mathbf{a}}_{53} \\
\underline{\mathbf{a}}_{20}
\end{array}\right] \quad \underline{\mathbf{b}}=\left[\begin{array}{c}
b_{5} \\
b_{4} \\
b_{3} \\
\hline b_{2} \\
b_{1} \\
b_{0}
\end{array}\right]=\left[\begin{array}{l}
\underline{\mathbf{b}}_{53} \\
\hline \underline{\mathbf{b}}_{20}
\end{array}\right] \\
& \underline{\mathbf{x}}(t)=\left\{\begin{array}{llll}
\underline{\mathbf{x}}_{1}(t)=\underline{\mathbf{F}}_{53}(t) & \underline{\mathbf{a}}_{53}+\underline{\mathbf{F}}_{20}(t) & \cdot \underline{\mathbf{a}}_{20} & 0 \leq t<t_{1} \\
\underline{\mathbf{x}}_{2}(t)=\underline{\mathbf{F}}_{53}\left(t-t_{1}\right) \cdot \underline{\mathbf{b}}_{53}+\underline{\mathbf{F}}_{20}\left(t-t_{1}\right) \cdot \underline{\mathbf{b}}_{20} & t_{1} \leq t \leq t_{2}
\end{array}\right.
\end{aligned}
$$

\section{Randbedingungen zum Zeitpunkt $t=0$}

Am Beginn der Trajektorie $t=0$ wird ein stetiger Übergang der Größen $p, v$ und $a$ gefordert. Diese Nebenbedingungen lassen sich auf Basis des bisherigen Zustandes $\underline{\mathbf{x}}_{0}$ wie folgt darstellen:

$$
\underline{\mathbf{C}}_{0} \underline{\mathbf{x}}_{1}(0)=\underline{\mathbf{C}}_{0} \underline{\mathbf{x}}_{0} \quad \text { mit } \quad \underline{\mathbf{C}}_{0}=\left[\begin{array}{cccc}
1 & 0 & 0 & 0 \\
0 & 1 & 0 & 0 \\
0 & 0 & 1 & 0
\end{array}\right]
$$

Setzt man $\underline{\mathbf{x}}_{1}(t)$ aus Gleichung (A.4) in die obige Gleichung ein, so lässt sich $\underline{\mathbf{a}}_{20}$ bestimmen:

$$
\begin{aligned}
\underbrace{}_{\underline{\mathbf{C}} \cdot \underline{\mathbf{F}}_{53}(0) \cdot \underline{\mathbf{a}}_{53}+\underline{\mathbf{C}}_{0} \cdot \underline{\mathbf{F}}_{20}(0) \cdot \underline{\mathbf{a}}_{20}} & =\underline{\mathbf{C}}_{0} \cdot \underline{\mathbf{x}}_{0} \\
\underline{\mathbf{C}}_{0} \cdot \underline{\mathbf{F}}_{20}(0) \cdot \underline{\mathbf{a}}_{20} & =\underline{\mathbf{C}}_{0} \cdot \underline{\mathbf{x}}_{0} \\
\underline{\mathbf{a}}_{20} & =\left(\underline{\mathbf{C}}_{0} \cdot \underline{\mathbf{F}}_{20}(0)\right)^{-1} \cdot \underline{\mathbf{C}}_{0} \cdot \underline{\mathbf{x}}_{0}
\end{aligned}
$$

Die Matrix $\underline{\mathbf{C}}_{0} \cdot \underline{\mathbf{F}}_{20}(0)$ hat dabei vollen Rang und ist somit invertierbar:

$$
\underline{\mathbf{C}}_{0} \cdot \underline{\mathbf{F}}_{20}(0)=\left[\begin{array}{ccc}
0 & 0 & 1 \\
0 & 1 & 0 \\
2 & 0 & 0
\end{array}\right] \quad\left(\underline{\mathbf{C}}_{0} \cdot \underline{\mathbf{F}}_{20}(0)\right)^{-1}=\left[\begin{array}{ccc}
0 & 0 & 0,5 \\
0 & 1 & 0 \\
1 & 0 & 0
\end{array}\right]
$$

Der Vektor $\underline{\mathbf{a}}_{20}$ ist daher nur von dem Startzustand $\underline{\mathbf{x}}_{0}$ abhängig. $\underline{\mathbf{a}}_{53}$ ist frei wählbar:

$$
\underline{\mathbf{a}}_{20}=\left[\begin{array}{cccc}
0 & 0 & 0,5 & 0 \\
0 & 1 & 0 & 0 \\
1 & 0 & 0 & 0
\end{array}\right] \cdot \underline{\mathbf{x}}_{0}
$$




\section{Randbedingungen zum Zeitpunkt $t_{1}$}

Auch zum Zeitpunkt $t_{1}$ wird ein stetiger Übergang von $p, v$, und $a$ gefordert. Das Vorgehen ist analog zum Zeitpunkt $t=0$ :

$$
\begin{aligned}
& \underline{\mathbf{C}}_{0} \cdot \underline{\mathbf{x}}_{2}\left(t_{1}\right)=\underline{\mathbf{C}}_{0} \cdot \underline{\mathbf{x}}_{1}\left(t_{1}\right) \\
& \begin{aligned}
\underbrace{\mathbf{C}_{0} \cdot \underline{\mathbf{F}}_{53}\left(t_{1}-t_{1}\right)}_{0} \cdot \underline{\mathbf{b}}_{53}+\underline{\mathbf{C}}_{0} \cdot \underline{\mathbf{F}}_{20}\left(t_{1}-t_{1}\right) \cdot \underline{\mathbf{b}}_{20} & =\underline{\mathbf{C}}_{0} \cdot \underline{\mathbf{F}}_{53}\left(t_{1}\right) \cdot \underline{\mathbf{a}}_{53}+\underline{\mathbf{C}}_{0} \cdot \underline{\mathbf{F}}_{20}\left(t_{1}\right) \cdot \underline{\mathbf{a}}_{20} \\
\underline{\mathbf{C}}_{0} \cdot \underline{\mathbf{F}}_{20}(0) \cdot \underline{\mathbf{b}}_{20} & =\underline{\mathbf{C}}_{0} \cdot \underline{\mathbf{F}}_{53}\left(t_{1}\right) \cdot \underline{\mathbf{a}}_{53}+\underline{\mathbf{C}}_{0} \cdot \underline{\mathbf{F}}_{20}\left(t_{1}\right) \cdot \underline{\mathbf{a}}_{20}
\end{aligned} \\
& \underline{\mathbf{b}}_{20}=\underbrace{\left(\mathbf{C}_{0} \cdot \underline{\mathbf{F}}_{20}(0)\right)^{-1} \cdot \underline{\mathbf{C}}_{0} \cdot \underline{\mathbf{F}}_{53}\left(t_{1}\right)}_{\underline{\mathbf{H}}_{253}} \cdot \underline{\mathbf{a}}_{53}+\underbrace{\left(\underline{\mathbf{C}}_{0} \cdot \underline{\mathbf{F}}_{20}(0)\right)^{-1} \cdot \underline{\mathbf{C}}_{0} \cdot \underline{\mathbf{F}}_{20}\left(t_{1}\right)}_{\underline{\mathbf{H}}_{220}} \cdot \underline{\mathbf{a}}_{20} \\
& \underline{\mathbf{b}}_{20}=\underline{\mathbf{H}}_{253}\left(t_{1}\right) \cdot \underline{\mathbf{a}}_{53}+\underline{\mathbf{H}}_{220}\left(t_{1}\right) \cdot \underline{\mathbf{a}}_{20}
\end{aligned}
$$

Die Parameter $\underline{\mathbf{b}}_{20}$ ergeben sich daher aus den Parametern des ersten Segmentes. Die Matrizen $\underline{\mathbf{H}}_{253}$ und $\underline{\mathbf{H}}_{220}$ hängen hierbei vom Zeitpunkt $t_{1}$ ab.

\section{Einsetzen in $\underline{x}(t)$}

Abschließend werden $\underline{\mathbf{a}}_{20}$ und $\underline{\mathbf{b}}_{20}$ in die Gleichungen für $\underline{\mathbf{x}}_{1}(t)$ sowie $\underline{\mathbf{x}}_{2}(t)$ (A.4) eingesetzt. Die freien Parameter werden im Vektor $\underline{\mathbf{v}}$ zusammengefasst:

$$
\begin{aligned}
& \underline{\mathbf{x}}_{1}(t)=\underline{\mathbf{F}}_{53}(t) \cdot \underline{\mathbf{a}}_{53}+\underline{\mathbf{F}}_{20}(t) \cdot \underline{\mathbf{a}}_{20} \\
& =\underbrace{\left[\begin{array}{ll}
\underline{\mathbf{F}}_{53}(t) & \mathbf{0}
\end{array}\right]}_{\underline{\mathbf{G}}_{1}(t)} \underbrace{\left[\begin{array}{c}
\underline{\mathbf{a}}_{53} \\
\underline{\mathbf{b}}_{53}
\end{array}\right]}_{\underline{\mathbf{v}}}+\underbrace{\mathbf{F}_{20}(t) \cdot \underline{\mathbf{a}}_{20}}_{\underline{\mathbf{e}}_{1}(t)} \\
& =\underline{\mathbf{G}}_{1}(t) \cdot \underline{\mathbf{v}}+\underline{\mathbf{e}}_{1}(t) \\
& \underline{\mathbf{x}}_{2}(t)=\underline{\mathbf{F}}_{53}\left(t-t_{1}\right) \cdot \underline{\mathbf{b}}_{53}+\underline{\mathbf{F}}_{20}\left(t-t_{1}\right) \cdot \underline{\mathbf{b}}_{20} \\
& \underline{\mathbf{x}}_{2}(t)=\underline{\mathbf{F}}_{53}\left(t-t_{1}\right) \cdot \underline{\mathbf{b}}_{53}+\underline{\mathbf{F}}_{20}\left(t-t_{1}\right) \cdot \underline{\mathbf{H}}_{253} \cdot \underline{\mathbf{a}}_{53}+\underline{\mathbf{F}}_{20}\left(t-t_{1}\right) \cdot \underline{\mathbf{H}}_{220} \cdot \underline{\mathbf{a}}_{20} \\
& =\underbrace{\left[\begin{array}{ll}
\underline{\mathbf{F}}_{20}\left(t-t_{1}\right) \cdot \underline{\mathbf{H}}_{253} & \underline{\mathbf{F}}_{53}\left(t-t_{1}\right)
\end{array}\right]}_{\underline{\mathbf{G}}_{2}(t)} \underbrace{\left[\begin{array}{c}
\underline{\mathbf{a}}_{53} \\
\underline{\mathbf{b}}_{53}
\end{array}\right]}_{\underline{\mathbf{v}}}+\underbrace{\mathbf{F}_{20}\left(t-t_{1}\right) \cdot \underline{\mathbf{H}}_{220} \cdot \underline{\mathbf{a}}_{20}}_{\underline{\mathbf{e}}_{2}(t)} \\
& =\underline{\mathbf{G}}_{2}(t) \cdot \underline{\mathbf{v}}+\underline{\mathbf{e}}_{2}(t)
\end{aligned}
$$

Somit erhält man folgende kompakte Darstellung in Abhängigkeit von v:

$$
\underline{\mathbf{x}}(t)= \begin{cases}\underline{\mathbf{x}}_{1}(t)=\underline{\mathbf{G}}_{1}(t) \cdot \underline{\mathbf{v}}+\underline{\mathbf{e}}_{1}(t) & 0 \leq t<t_{1} \\ \underline{\mathbf{x}}_{2}(t)=\underline{\mathbf{G}}_{2}(t) \cdot \underline{\mathbf{v}}+\underline{\mathbf{e}}_{2}(t) & t_{1} \leq t \leq t_{2}\end{cases}
$$

Die Matrizen hängen neben der Zeit $t$ noch vom Anfangszustand $\underline{\mathbf{x}}_{0}$ sowie dem Zeitpunkt $t_{1}$ ab. Sie wurden analytisch mit Hilfe der Matlaв Symbolic Math Toolbox bestimmt und werden in den folgenden Abschnitten weiter verwendet. Abschließend werden die dann auftauchenden Matrizen automatisiert mit Hilfe des Befehls matlabFunction in rechenzeitoptimierten MATLAB Quelltext umgewandelt, der für die C-Code Generierung geeignet ist. 


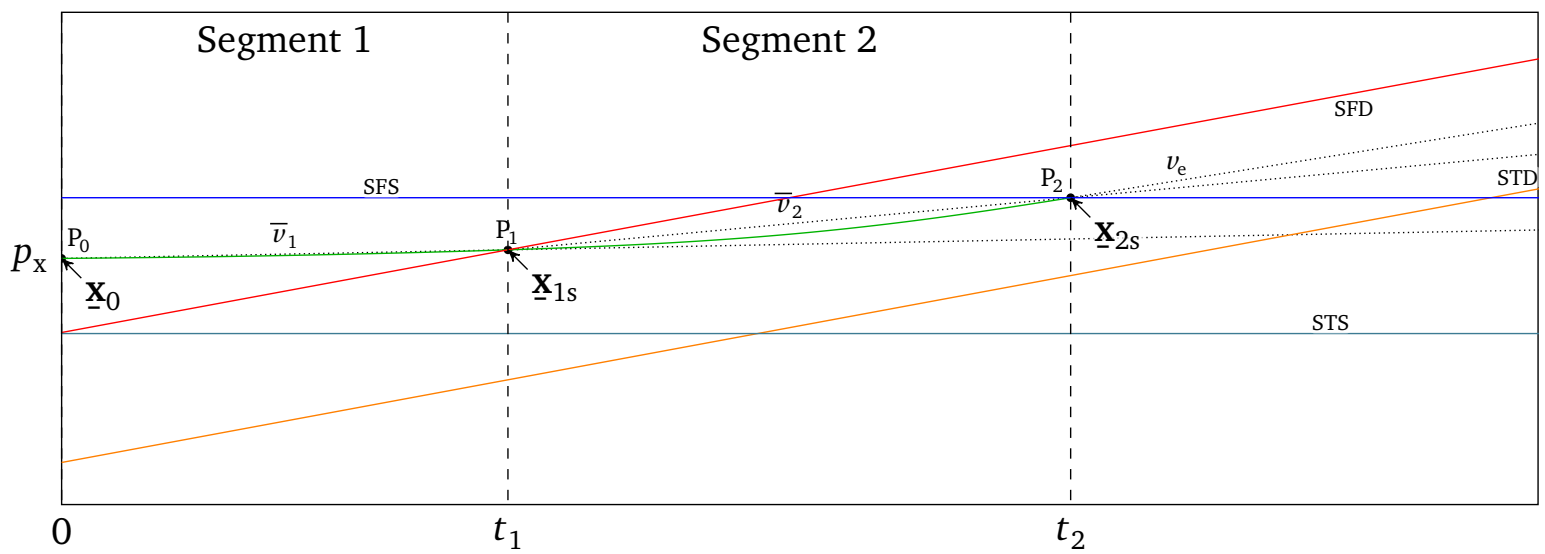

Abbildung A.1: Beispielhafte Längstrajektorie (grün) bestehend aus zwei Polynomen 5. Grades zusammen mit vier möglichen Umfeldfahrzeugen

Die Längstrajektorie besteht, wie in Abbildung A.1 dargestellt, aus zwei Polynomen fünften Grades. In Abschnitt A.1 wurde gezeigt, dass sich die Trajektorie wie folgt beschreiben lässt:

$$
\underline{\mathbf{x}}(t)= \begin{cases}\underline{\mathbf{x}}_{1}(t)=\underline{\mathbf{G}}_{1}(t) \cdot \underline{\mathbf{v}}+\underline{\mathbf{e}}_{1}(t) & 0 \leq t<t_{1} \\ \underline{\mathbf{x}}_{2}(t)=\underline{\mathbf{G}}_{2}(t) \cdot \underline{\mathbf{v}}+\underline{\mathbf{e}}_{2}(t) & t_{1} \leq t \leq t_{2}\end{cases}
$$

Wie in Abschnitt 4.3.1 auf Seite 29 beschrieben, soll das Integral über den quadrierten Ruck minimiert werden:

$$
\underset{\underline{\mathbf{v}}}{\arg \min } \int_{0}^{t_{2}} j_{\mathrm{x}}^{2}(\underline{\mathbf{v}}, t) \mathrm{d} t
$$

Hierbei müssen lineare Nebenbedingungen der Form

$$
\underline{\mathbf{C}} \cdot \underline{\mathbf{v}}=\underline{\mathbf{d}}
$$

berücksichtigt werden. Zu Testzwecken war es aber auch gewünscht, andere Zustandsgrößen zu gewichten. Daher wird eine etwas allgemeinere Darstellung mit dem Zustandsvektor $\underline{\mathbf{x}}$ und einer Gewichtungsmatrix gewählt.

Für den Punkt $\mathrm{P}_{1}$ ist die Position und für den Punkt $\mathrm{P}_{2}$ sind Position, Geschwindigkeit und Beschleunigung vorgegeben (siehe Abschnitt 4.3.1). Position und Geschwindigkeit werden dabei über harte Nebenbedingungen berücksichtigt, worauf am Ende dieses Abschnittes eingegangen wird. Für die Beschleunigung hat es sich als sinnvoll erwiesen, diese nicht hart vorzugeben, sondern sie über einen Term in der Gütefunktion zu berücksichtigen. Dieser bewertet die Abweichung der Endbeschleunigung vom vorgegebenen Wert. Wäre die Beschleunigung fest vorgegeben, so würde das in der Praxis aufgrund von Rauschen und Fehlern in der Umfeldsensorik zu Problemen führen. Dies gilt insbesondere dann, wenn die vorgegebene Beschleunigung in der verbleibenden Zeit nicht erreicht werden kann. 


\section{Güte}

Mit $\underline{\mathbf{x}}_{2 \mathrm{~s}}=\left[\begin{array}{llll}p_{2} & v_{\mathrm{e}} & a_{\mathrm{e}} & 0\end{array}\right]^{\mathrm{T}}$ als Soll-Endzustand ergibt sich die Güte der Längstrajektorie zu:

$$
J_{\text {Lon }}=\underbrace{\int_{0}^{t_{1}}\left[\underline{\mathbf{x}}_{1}^{\mathrm{T}}(t) \underline{\mathbf{W}}_{1} \underline{\mathbf{x}}_{1}(t)\right] \mathrm{d} t}_{J_{1}}+\underbrace{\int_{t_{1}}^{t_{2}}\left[\underline{\mathbf{x}}_{2}^{\mathrm{T}}(t) \underline{\mathbf{W}}_{2} \underline{\mathbf{x}}_{2}(t)\right] \mathrm{d} t}_{J_{2}}+\underbrace{\left(\underline{\mathbf{x}}_{2}^{\mathrm{T}}\left(t_{2}\right)-\underline{\mathbf{x}}_{2 s}^{\mathrm{T}}\right) \underline{\mathbf{W}}_{3}\left(\underline{\mathbf{x}}_{2}\left(t_{2}\right)-\underline{\mathbf{x}}_{2 s}\right)}_{J_{3}}
$$

Die Matrizen $\underline{\mathbf{W}}_{1}$ und $\underline{\mathbf{W}}_{2}$ enthalten hierbei nur Einträge für den Ruck, die Matrix $\underline{\mathbf{W}}_{3}$ nur Einträge für die Beschleunigung. Im Folgenden werden die einzelnen Komponenten genauer betrachtet.

\section{Güte im 1. Segment}

Einsetzen von $\underline{x}_{1}(t)$ aus Gleichung (A.10) in $J_{1}$ liefert:

$$
\begin{aligned}
J_{1} & =\int_{0}^{t_{1}}\left[\left(\underline{\mathbf{v}}^{\mathrm{T}} \underline{\mathbf{G}}_{1}^{\mathrm{T}}(t)+\underline{\mathbf{e}}_{1}^{\mathrm{T}}(t)\right) \underline{\mathbf{W}}_{1}\left(\underline{\mathbf{G}}_{1}(t) \underline{\mathbf{v}}+\underline{\mathbf{e}}_{1}(t)\right)\right] \mathrm{d} t \\
& =\underline{\mathbf{v}}^{\mathrm{T}} \underbrace{\int_{0}^{t_{1}}\left[\underline{\mathbf{G}}_{1}^{\mathrm{T}}(t) \underline{\mathbf{W}}_{1} \underline{\mathbf{G}}_{1}(t)\right] \mathrm{d} t}_{\underline{\mathbf{W}}_{11}} \underline{\mathbf{v}}+\underbrace{\int_{0}^{t_{1}}\left[2 \underline{\mathbf{e}}_{1}^{\mathrm{T}}(t) \underline{\mathbf{W}}_{1} \underline{\mathbf{G}}_{1}(t)\right] \mathrm{d} t}_{\underline{\mathbf{w}}_{12}^{\mathrm{T}}}+\underbrace{\int_{0}^{t_{1}}\left[\underline{\mathbf{e}}_{1}^{\mathrm{T}}(t) \underline{\mathbf{W}}_{1} \underline{\mathbf{e}}_{1}(t)\right] \mathrm{d} t}_{w_{13}} \\
& =\underline{\mathbf{v}}^{\mathrm{T}} \underline{\mathbf{W}}_{11} \underline{\mathbf{v}}+\underline{\mathbf{w}}_{12}^{\mathrm{T}} \underline{\mathbf{v}}+w_{13}
\end{aligned}
$$

\section{Güte im 2. Segment}

Einsetzen von $\underline{\mathbf{x}}_{2}(t)$ aus Gleichung (A.10) in $J_{2}$ liefert:

$$
\begin{aligned}
J_{2} & =\int_{t_{1}}^{t_{2}}\left[\left(\underline{\mathbf{v}}^{\mathrm{T}} \underline{\mathbf{G}}_{2}^{\mathrm{T}}(t)+\underline{\mathbf{e}}_{2}^{\mathrm{T}}(t)\right) \underline{\mathbf{w}}_{2}\left(\underline{\mathbf{G}}_{2}(t) \underline{\mathbf{v}}+\underline{\mathbf{e}}_{2}(t)\right)\right] \mathrm{d} t \\
& =\underline{\mathbf{v}}^{\mathrm{T}} \underbrace{\int_{t_{1}}^{t_{2}}\left[\underline{\mathbf{G}}_{2}^{\mathrm{T}}(t) \underline{\mathbf{W}}_{2} \underline{\mathbf{G}}_{2}(t)\right] \mathrm{d} t}_{\underline{\mathbf{W}}_{21}} \underline{\mathbf{v}}+\underbrace{\int_{t_{1}}^{t_{2}}\left[2 \underline{\mathbf{e}}_{2}^{\mathrm{T}}(t) \underline{\mathbf{W}}_{2} \underline{\mathbf{G}}_{2}(t)\right] \mathrm{d} t}_{\underline{\mathbf{w}}_{22}^{\mathrm{T}}}+\underbrace{\int_{t_{1}}^{t_{2}}\left[\underline{\mathbf{e}}_{2}^{\mathrm{T}}(t) \underline{\mathbf{w}}_{2} \underline{\mathbf{e}}_{2}(t)\right] \mathrm{d} t}_{w_{23}} \\
& =\underline{\mathbf{v}}^{\mathrm{T}} \underline{\mathbf{W}}_{21} \underline{\mathbf{v}}+\underline{\mathbf{w}}_{22}^{\mathrm{T}} \underline{\mathbf{v}}+w_{23}
\end{aligned}
$$

\section{Güte des Endpunkts}

$$
J_{3}=\underbrace{\left(\underline{\mathbf{x}}_{2}^{\mathrm{T}}\left(t_{2}\right)-\underline{\mathbf{x}}_{2 \mathrm{~s}}^{\mathrm{T}}\right)}_{\mathbf{z}_{0}^{\mathrm{T}}} \underline{\mathbf{W}}_{3} \underbrace{\left(\underline{\mathbf{x}}_{2}\left(t_{2}\right)-\underline{\mathbf{x}}_{2 \mathrm{~s}}\right)}_{\underline{\mathbf{z}}_{0}}
$$




$$
\begin{aligned}
\underline{\mathbf{z}}_{0} & =\underbrace{\mathbf{G}_{2}\left(t_{2}\right)}_{\underline{\mathbf{G}}_{3}} \underline{\mathbf{v}}+\underbrace{\mathbf{e}_{2}\left(t_{2}\right)-\underline{\mathbf{x}}_{2 s}}_{\underline{\mathbf{e}}_{3}} \\
& =\underline{\mathbf{G}}_{3} \underline{\mathbf{v}}+\underline{\mathbf{e}}_{3} \\
J_{3} & =\left[\underline{\mathbf{v}}^{\mathrm{T}} \mathbf{G}_{3}^{\mathrm{T}}+\underline{\mathbf{e}}_{3}^{\mathrm{T}}\right] \underline{\mathbf{W}}_{3}\left[\underline{\mathbf{G}}_{3} \underline{\mathbf{v}}+\underline{\mathbf{e}}_{3}\right] \\
& =\underline{\mathbf{v}}^{\mathrm{T}} \underbrace{\underline{\mathbf{v}}}_{\underline{\mathbf{G}}_{3}^{\mathrm{T}} \underline{\mathbf{W}}_{3} \underline{\mathbf{G}}_{3}}+\underbrace{2 \underline{\mathbf{e}}_{3}^{\mathrm{T}} \underline{\mathbf{W}}_{3} \underline{\mathbf{G}}_{3}}_{\underline{\mathbf{w}}_{32}^{\mathrm{T}}} \underline{\mathbf{v}}+\underbrace{\underline{\mathbf{e}}_{3}^{\mathrm{T}} \underline{\mathbf{W}}_{3} \mathbf{e}_{3}}_{w_{33}} \\
& =\underline{\mathbf{v}}^{\mathrm{T}} \underline{\mathbf{W}}_{31} \underline{\mathbf{v}}+\underline{\mathbf{w}}_{32}^{\mathrm{T}} \underline{\mathbf{v}}+w_{33}
\end{aligned}
$$

\section{Gesamtgüte}

Werden die Güteterme für die drei Segmente addiert so erhält man:

$$
\begin{aligned}
J_{\mathrm{Lon}} & =\underline{\mathbf{v}}^{\mathrm{T}} \underbrace{\left(\underline{\mathbf{W}}_{11}+\underline{\mathbf{W}}_{21}+\underline{\mathbf{W}}_{31}\right)}_{\underline{\mathbf{W}}_{\mathrm{G} 1}} \underline{\mathbf{v}}+\underbrace{\left(\underline{\mathbf{w}}_{12}^{\mathrm{T}}+\underline{\mathbf{w}}_{22}^{\mathrm{T}}+\underline{\mathbf{w}}_{32}^{\mathrm{T}}\right)}_{\underline{\mathbf{w}}_{\mathrm{G} 2}^{\mathrm{T}}} \underline{\mathbf{v}}+\underbrace{\left(w_{13}+w_{23}+w_{33}\right)}_{w_{\mathrm{G} 3}} \\
& =\underline{\mathbf{v}}^{\mathrm{T}} \underline{\mathbf{W}}_{\mathrm{G} 1} \underline{\mathbf{v}}+\underline{\mathbf{w}}_{\mathrm{G} 2}^{\mathrm{T}} \underline{\mathbf{v}}+w_{\mathrm{G} 3}
\end{aligned}
$$

\section{Nebenbedingungen}

Als zusätzliche Nebenbedingungen werden die Position zum Zeitpunkt $t_{1}$ sowie Position und Geschwindigkeit zum Zeitpunkt $t_{2}$ vorgegeben (Abbildung A.1):

$$
\begin{aligned}
& \underline{\mathbf{x}}_{1 s}=\left[\begin{array}{llll}
p_{1} & 0 & 0 & 0
\end{array}\right]^{\mathrm{T}} \\
& \underline{\mathbf{x}}_{2 s}=\left[\begin{array}{llll}
p_{2} & v_{\mathrm{e}} & a_{\mathrm{e}} & 0
\end{array}\right]^{\mathrm{T}} \\
& \underline{\mathbf{C}}_{\mathrm{P}}=\left[\begin{array}{llll}
1 & 0 & 0 & 0
\end{array}\right] \\
& \underline{\mathbf{C}}_{\mathrm{N}}=\left[\begin{array}{llll}
1 & 0 & 0 & 0 \\
0 & 1 & 0 & 0
\end{array}\right] \\
& \underline{\mathbf{C}}_{\mathrm{P}} \underline{\mathbf{x}}_{1}\left(t_{1}\right)=\underline{\mathbf{C}}_{\mathrm{N}} \underline{\mathbf{x}}_{1 s} \\
& \underline{\mathbf{C}}_{\mathrm{N}} \underline{\mathbf{x}}_{2}\left(t_{2}\right)=\underline{\mathbf{C}}_{\mathrm{N}} \underline{\mathbf{x}}_{2 s}
\end{aligned}
$$

Einsetzen von $\underline{\mathbf{x}}_{1}(t)$ und $\underline{\mathbf{x}}_{2}(t)$ aus Gleichung (A.10) liefert:

$$
\begin{aligned}
& \underline{\mathbf{C}}_{\mathrm{N}}\left[\underline{\mathbf{G}}_{2}\left(t_{2}\right) \cdot \underline{\mathbf{v}}+\underline{\mathbf{e}}_{2}\left(t_{2}\right)\right]=\underline{\mathbf{C}}_{N} \underline{\mathbf{x}}_{2 \mathrm{~s}} \\
& \underline{\mathbf{C}}_{\mathrm{P}}\left[\underline{\mathbf{G}}_{1}\left(t_{1}\right) \cdot \underline{\mathbf{v}}+\underline{\mathbf{e}}_{1}\left(t_{1}\right)\right]=\underline{\mathbf{C}}_{P} \underline{\mathbf{x}}_{1 \mathrm{~s}}
\end{aligned}
$$

Dies lässt sich in folgende Matrix-Form umformen:

$$
\begin{aligned}
& \underbrace{\left[\begin{array}{l}
\underline{\mathbf{C}}_{\mathrm{N}} \underline{\mathbf{G}}_{2}\left(t_{2}\right) \\
\underline{\mathbf{C}}_{\mathrm{P}} \underline{\mathbf{G}}_{1}\left(t_{1}\right)
\end{array}\right]}_{\underline{\mathbf{C}}} \underline{\mathbf{v}}=\underbrace{\left[\begin{array}{l}
\underline{\mathbf{C}}_{\mathrm{N}} \underline{\mathbf{x}}_{2 \mathrm{~s}}-\underline{\mathbf{C}}_{\mathrm{N}} \mathbf{e}_{2}\left(t_{2}\right) \\
\underline{\mathbf{C}}_{\mathrm{P}} \underline{\mathbf{x}}_{1 \mathrm{~s}}-\underline{\mathbf{C}}_{\mathrm{N}} \underline{\mathbf{e}}_{1}\left(t_{1}\right)
\end{array}\right]}_{\underline{\mathbf{d}}} \\
& \underline{\mathbf{C v}}=\underline{\mathbf{d}}
\end{aligned}
$$




\section{Lösung des Optimierungsproblems}

Die optimalen Parameter für die Minimierung von $J_{\text {Lon }}$ erhält man aus der Lösung von folgendem Gleichungssystem (siehe auch Anhang E):

$$
\left[\begin{array}{cc}
2 \underline{\mathbf{W}}_{\mathrm{G} 1} & \underline{\mathbf{C}}^{\mathrm{T}} \\
\underline{\mathbf{C}} & \underline{\mathbf{0}}
\end{array}\right]\left[\begin{array}{l}
\underline{\mathbf{v}} \\
\underline{\lambda}
\end{array}\right]=\left[\begin{array}{c}
-\underline{\mathbf{w}}_{\mathrm{G} 2} \\
\underline{\mathbf{d}}
\end{array}\right]
$$

$\mathrm{Zu}$ beachten ist, dass $\underline{\mathbf{W}}_{\mathrm{G} 1}, \underline{\mathbf{w}}_{\mathrm{G} 2}$ sowie $\underline{\mathbf{C}}$ und $\underline{\mathbf{d}}$ von den Größen $t_{1}, t_{2}, \underline{\mathbf{x}}_{0}, \underline{\mathbf{x}}_{1 \mathrm{~s}}$ und $\underline{\mathbf{x}}_{2 \mathrm{~s}}$ abhängig sind. Die Matrizen werden, in Abhängigkeit dieser Größen, von den in Abschnitt A.1 beschriebenen MaтLAв-Funktionen auf eine möglichst rechenzeitoptimale Weise erzeugt. Anschließend kann das lineare Gleichungssystem leicht durch den Prozessor gelöst werden.

\section{Parameter}

Für die Versuchsfahrten wurden folgende Parameter verwendet:

$$
\underline{\mathbf{W}}_{1}=\left[\begin{array}{cccc}
0 & 0 & 0 & 0 \\
0 & 0 & 0 & 0 \\
0 & 0 & 0 & 0 \\
0 & 0 & 0 & 1
\end{array}\right] \quad \underline{\mathbf{W}}_{2}=\left[\begin{array}{cccc}
0 & 0 & 0 & 0 \\
0 & 0 & 0 & 0 \\
0 & 0 & 0 & 0 \\
0 & 0 & 0 & 1
\end{array}\right] \quad \underline{\mathbf{W}}_{3}=\left[\begin{array}{cccc}
0 & 0 & 0 & 0 \\
0 & 0 & 0 & 0 \\
0 & 0 & 1000 & 0 \\
0 & 0 & 0 & 0
\end{array}\right]
$$

Somit gehen nur die Rucke während den ersten zwei Segmenten sowie die Soll-Ist-Abweichung der Beschleunigung am Endpunkt in das Gütemaß ein. 


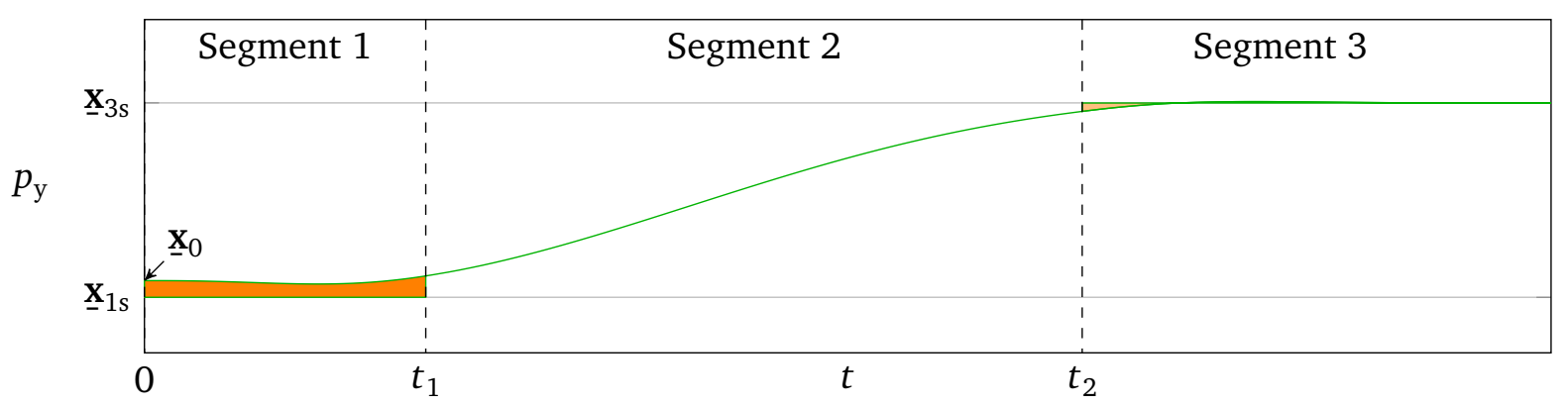

Abbildung A.2: Quertrajektorie der Drei-Segment-Planung mit Darstellung der Strafterme im ersten und dritten Segment

In Abschnitt 4.3.2 wurde das Gütefunktional für die einzelnen Segmente der Quertrajektorie vorgestellt. In den ersten beiden Segmenten besteht die Trajektorie aus Polynomen 5. Grades. Im dritten Segment wird die Trajektorie durch eine Matrix-Exponentialfunktion beschrieben (Abbildung A.2). Dabei handelt es sich, wie sich später zeigen wird, um den optimalen Verlauf für das Gütekriterium dieses Segmentes. Unter Verwendung der Vereinfachungen aus Abschnitt A.1 ergibt sich die Quertrajektorie somit zu:

$$
\underline{\mathbf{x}}(t)= \begin{cases}\underline{\mathbf{x}}_{1}(t)=\underline{\mathbf{G}}_{1}(t) \cdot \underline{\mathbf{v}}+\underline{\mathbf{e}}_{1}(t) & 0 \leq t<t_{1} \\ \underline{\mathbf{x}}_{2}(t)=\underline{\mathbf{G}}_{2}(t) \cdot \underline{\mathbf{v}}+\underline{\mathbf{e}}_{2}(t) & t_{1} \leq t \leq t_{2} \\ \underline{\mathbf{x}}_{3}(t) & t_{2}<t\end{cases}
$$

In Abschnitt A.2 wird gezeigt, dass sich die Güte des dritten Segmentes leicht aus dem Endzustand zum Zeitpunkt $t_{2}$ bestimmen lässt. Auf diese Weise lässt sich das Segment einfach in die Optimierung integrieren. So ist abschließend nur ein lineares Gleichungssystem mit 6 Unbekannten zu lösen.

\section{Güte}

In Matrixform lautet das Gütekriterium für die drei Segmente wie folgt (siehe auch Abbildung A.2):

$$
\begin{aligned}
J_{\text {Lat }}= & \underbrace{\int_{0}^{t_{1}}\left[\left(\underline{\mathbf{x}}_{1}^{\mathrm{T}}(t)-\underline{\mathbf{x}}_{1 \mathrm{~s}}^{\mathrm{T}}\right) \underline{\mathbf{w}}_{1}\left(\underline{\mathbf{x}}_{1}(t)-\underline{\mathbf{x}}_{1 \mathrm{~s}}\right)\right] \mathrm{d} t}_{J_{1}}+\underbrace{\int_{t_{1}}^{t_{2}}\left[\underline{\mathbf{x}}_{2}^{\mathrm{T}}(t) \underline{\mathbf{W}}_{2} \underline{\mathbf{x}}_{2}(t)\right] \mathrm{d} t}_{J_{2}}+ \\
& \underbrace{\int_{t_{2}}^{\infty}\left[\left(\underline{\mathbf{x}}_{3}^{\mathrm{T}}(t)-\underline{\mathbf{x}}_{3 \mathrm{~s}}^{\mathrm{T}}\right) \underline{\mathbf{W}}_{3}\left(\underline{\mathbf{x}}_{3}(t)-\underline{\mathbf{x}}_{3 \mathrm{~s}}\right)\right] \mathrm{d} t}_{J_{3}} t
\end{aligned}
$$

Die Matrizen $\underline{\mathbf{W}}_{1}, \underline{\mathbf{W}}_{2}$ und $\underline{\mathbf{W}}_{3}$ sind hierbei diagonale Gewichtungsmatrizen, deren Werte in Abschnitt A.3 aufgeführt sind. Die Gleichungen werden nun so umgeformt, dass sich ein quadratisches Optimierungsproblem ergibt. 


\section{Segment}

Durch Ausmultiplizieren von $J_{1}$ erhält man:

$$
J_{1}=\underbrace{\int_{0}^{t_{1}}\left[\underline{\mathbf{x}}_{1}^{\mathrm{T}}(t) \underline{\mathbf{W}}_{1} \underline{\mathbf{x}}_{1}(t)\right] \mathrm{d} t}_{J_{11}}+\underbrace{\int_{0}^{t_{1}}\left[-2 \underline{\mathbf{x}}_{1 s}^{\mathrm{T}} \underline{\mathbf{W}}_{1} \underline{\mathbf{x}}_{1}(t)\right] \mathrm{d} t}_{J_{12}}+\underbrace{\int_{0}^{t_{1}}\left[\underline{\mathbf{x}}_{1 s}^{\mathrm{T}} \underline{\mathbf{w}}_{1} \underline{\mathbf{x}}_{1 \mathrm{~s}}\right] \mathrm{d} t}_{J_{13}}
$$

Einsetzen von $\underline{\mathbf{x}}_{1}(t)$ aus Gleichung (A.22) liefert:

$$
\begin{aligned}
J_{11} & =\int_{0}^{t_{1}}\left[\underline{\mathbf{x}}_{1}^{\mathrm{T}}(t) \underline{\mathbf{W}}_{1} \underline{\mathbf{x}}_{1}(t)\right] \mathrm{d} t \\
& =\int_{0}^{t_{1}}\left[\left(\underline{\mathbf{v}}^{\mathrm{T}} \underline{\mathbf{G}}_{1}^{\mathrm{T}}(t)+\underline{\mathbf{e}}_{1}^{\mathrm{T}}(t)\right) \underline{\mathbf{W}}_{1}\left(\underline{\mathbf{G}}_{1}(t) \cdot \underline{\mathbf{v}}+\underline{\mathbf{e}}_{1}(t)\right)\right] \mathrm{d} t \\
& =\int_{0}^{t_{1}}\left[\underline{\mathbf{v}}^{\mathrm{T}} \underline{\mathbf{G}}_{1}^{\mathrm{T}}(t) \underline{\mathbf{W}}_{1} \underline{\mathbf{G}}_{1}(t) \underline{\mathbf{v}}\right] \mathrm{d} t+\int_{0}^{t_{1}}\left[2 \underline{\mathbf{e}}_{1}^{\mathrm{T}}(t) \underline{\mathbf{W}}_{1} \underline{\mathbf{G}}_{1}(t) \underline{\mathbf{v}}\right] \mathrm{d} t+\underbrace{\int_{0}^{t_{1}}\left[\underline{\mathbf{e}}_{1}^{\mathrm{T}}(t) \underline{\mathbf{W}}_{1} \underline{\mathbf{e}}_{1}^{\mathrm{T}}(t)\right] \mathrm{d} t}_{\underline{\mathbf{W}}_{111}} \\
& =\underline{\mathbf{v}}^{\mathrm{T}} \underbrace{\int_{0}^{t_{1}}\left[\underline{\mathbf{G}}_{1}^{\mathrm{T}}(t) \underline{\mathbf{W}}_{1} \underline{\mathbf{G}}_{1}(t)\right] \mathrm{d} t \underline{\mathbf{v}}}_{0}+\underbrace{\int_{0}^{t_{1}}\left[\underline{\mathbf{e}}_{1}^{\mathrm{T}}(t) \underline{\mathbf{W}}_{1} \underline{\mathbf{e}}_{1}(t)\right] \mathrm{d} t}_{w_{113}^{t_{1}}\left[2 \underline{\mathbf{e}}_{1}^{\mathrm{T}}(t) \underline{\mathbf{W}}_{1} \underline{\mathbf{G}}_{1}(t)\right] \mathrm{d} t} \\
& =\underline{\mathbf{v}}^{\mathrm{T}} \underline{\mathbf{W}}_{111} \underline{\mathbf{v}}+\underline{\mathbf{w}}_{112}^{\mathrm{T}} \underline{\mathbf{v}}+w_{113}
\end{aligned}
$$

$$
\begin{aligned}
J_{12} & =\int_{0}^{t_{1}}\left[-2 \underline{\mathbf{x}}_{1 s}^{\mathrm{T}}(t) \underline{\mathbf{w}}_{1} \underline{\mathbf{x}}_{1}(t)\right] \mathrm{d} t \\
& =\int_{0}^{t_{1}}\left[-2 \underline{\mathbf{x}}_{1 s}^{\mathrm{T}} \underline{\mathbf{w}}_{1}\left(\underline{\mathbf{G}}_{1}(t) \underline{\mathbf{v}}+\underline{\mathbf{e}}_{1}(t)\right)\right] \mathrm{d} t \\
& =\underbrace{\int_{0}^{t_{1}}\left[-2 \underline{\mathbf{x}}_{1 s}^{\mathrm{T}} \underline{\mathbf{w}}_{1} \mathbf{G}_{1}(t)\right] \mathrm{d} t}_{\underline{\mathbf{w}}_{121}^{\mathrm{T}}} \underline{\mathbf{v}}+\underbrace{\int_{0}^{t_{1}}\left[-2 \underline{\mathbf{x}}_{1 s}^{\mathrm{T}} \underline{\mathbf{w}}_{1} \underline{\mathbf{e}}_{1}(t)\right] \mathrm{d} t}_{w_{122}} \\
& =\underline{\mathbf{w}}_{121}^{\mathrm{T}} \underline{\mathbf{v}}+w_{122}
\end{aligned}
$$

$$
J_{13}=\underbrace{\int_{t_{0}}^{t_{1}}\left[\underline{\mathbf{x}}_{1 s}^{\mathrm{T}} \underline{\mathbf{W}}_{1} \underline{\mathbf{x}}_{1 s}\right] \mathrm{d} t}_{w_{131}}
$$

Somit ergibt sich die Güte im 1. Segment zu:

$$
J_{1}=\underline{\mathbf{v}}^{\mathrm{T}} \underline{\mathbf{W}}_{111} \underline{\mathbf{v}}+\left(\underline{\mathbf{w}}_{112}^{\mathrm{T}}+\underline{\mathbf{w}}_{121}^{\mathrm{T}}\right) \underline{\mathbf{v}}+\left(w_{113}+w_{122}+w_{131}\right)
$$




\section{Segment}

Einsetzen von $\underline{\mathbf{x}}_{2}(t)$ aus Gleichung (A.22) in $J_{2}$ liefert:

$$
\begin{aligned}
J_{2} & =\int_{t_{1}}^{t_{2}}\left[\underline{\mathbf{x}}_{2}^{\mathrm{T}}(t) \underline{\mathbf{W}}_{2} \underline{\mathbf{x}}_{2}\right] \mathrm{d} t \\
& =\int_{t_{1}}^{t_{2}}\left[\left(\underline{\mathbf{v}}^{\mathrm{T}} \underline{\mathbf{G}}_{2}^{\mathrm{T}}(t)+\underline{\mathbf{e}}_{2}^{\mathrm{T}}(t)\right) \underline{\mathbf{W}}_{2}\left(\underline{\mathbf{G}}_{2}(t) \underline{\mathbf{v}}+\underline{\mathbf{e}}_{2}(t)\right)\right] \mathrm{d} t \\
& =\int_{t_{1}}^{t_{2}}\left[\underline{\mathbf{v}}^{\mathrm{T}} \underline{\mathbf{G}}_{2}^{\mathrm{T}}(t) \underline{\mathbf{W}}_{2} \underline{\mathbf{G}}_{2}(t) \underline{\mathbf{v}}\right] \mathrm{d} t+\int_{t_{1}}^{t_{2}}\left[2 \underline{\mathbf{e}}_{2}^{\mathrm{T}}(t) \underline{\mathbf{W}}_{2} \underline{\mathbf{G}}_{2}(t) \underline{\mathbf{v}}\right] \mathrm{d} t+\underbrace{t_{2}}_{t_{1}}\left[\underline{\mathbf{e}}_{2}^{\mathrm{T}}(t) \underline{\mathbf{W}}_{2} \underline{\mathbf{e}}_{2}(t)\right] \mathrm{d} t \\
& =\underline{\mathbf{v}}^{\mathrm{T}} \underbrace{\int_{t_{1}}^{t_{2}}\left[\underline{\mathbf{G}}_{2}^{\mathrm{T}}(t) \underline{\mathbf{W}}_{2} \underline{\mathbf{G}}_{2}(t)\right] \mathrm{d} t}_{\underline{\mathbf{W}}_{21}} \underline{\underbrace{\mathrm{T}}_{22}}+\underbrace{\left.\int_{t_{1}}^{t_{2}}\left[\underline{\mathbf{e}}_{2}^{\mathrm{T}}(t) \underline{\mathbf{W}}_{2} \underline{\mathbf{G}}_{2}(t)\right] \mathrm{d} t \underline{\mathbf{W}}_{2} \underline{\mathbf{e}}_{2}(t)\right] \mathrm{d} t}_{t_{1}} \\
& =\underline{\mathbf{v}}^{\mathrm{T}} \underline{\mathbf{W}}_{21} \underline{\mathbf{v}}+\underline{\mathbf{w}}_{22}^{\mathrm{T}} \underline{\mathbf{v}}+w_{23}
\end{aligned}
$$

\section{Segment}

Das Gütekriterium für das 3. Segment lautet:

$$
J_{3}=\int_{t_{2}}^{\infty}\left[\left(\underline{\mathbf{x}}_{3}^{\mathrm{T}}(t)-\underline{\mathbf{x}}_{3 \mathrm{~s}}^{\mathrm{T}}\right) \underline{\mathbf{W}}_{3}\left(\underline{\mathbf{x}}_{3}(t)-\underline{\mathbf{x}}_{3 \mathrm{~s}}\right)\right] \mathrm{d} t
$$

Nach Anwendung der Transformation

$$
\underline{\mathbf{z}}(\tau)=\underline{\mathbf{x}}_{3}\left(\tau+t_{2}\right)-\underline{\mathbf{x}}_{3 \mathrm{~s}}
$$

erkennt man leicht, dass das sich ergebende Gütefunktional

$$
J_{3}=\int_{0}^{\infty}\left[\underline{\mathbf{z}}(\tau)^{\mathrm{T}} \underline{\mathbf{W}}_{3} \underline{\mathbf{z}}(\tau)\right] \mathrm{d} \tau
$$

durch einen Riccati-Regler minimiert wird [Föllinger, 2016, S. 339 ff.]. In Abschnitt A.2 wird gezeigt, dass sich $J_{3}$ direkt in Abhängigkeit des Anfangszustandes $\underline{\mathbf{z}}_{0}$ bestimmen lässt:

$$
J_{3}=\underline{\mathbf{z}}_{0}^{\mathrm{T}} \cdot \overline{\mathbf{W}}_{3} \cdot \underline{\mathbf{z}}_{0}
$$

Ebenfalls wird dort gezeigt, wie man von der Gewichtungsmatrix $\underline{\mathbf{W}}_{3}$ auf die Gewichtungsmatrix $\underline{\mathbf{W}}_{3}$ bzgl. des Anfangszustandes gelangt. Der Anfangszustand $\underline{\mathbf{z}}_{0}$ lässt sich wieder in Abhängigkeit vom Parametervektor $\underline{\mathbf{v}}$ darstellen:

$$
\begin{aligned}
\underline{\mathbf{z}}_{0} & =\underline{\mathbf{x}}_{2}\left(t_{2}\right)-\underline{\mathbf{x}}_{3 \mathrm{~s}} \\
\underline{\mathbf{z}}_{0} & =\underbrace{\mathbf{G}_{2}\left(t_{2}\right)}_{\mathbf{G}_{3}} \underline{\mathbf{v}}+\underbrace{\mathbf{e}_{2}\left(t_{2}\right)-\underline{\mathbf{x}}_{3 s}}_{\underline{\mathbf{e}}_{3}} \\
& =\underline{\mathbf{G}}_{3} \cdot \underline{\mathbf{v}}+\underline{\mathbf{e}}_{3}
\end{aligned}
$$


Durch diese Ersetzung ergibt sich für $J_{3}$ :

$$
\begin{aligned}
J_{3} & =\left(\underline{\mathbf{v}}^{\mathrm{T}} \underline{\mathbf{G}}_{3}^{\mathrm{T}}+\underline{\mathbf{e}}_{3}^{\mathrm{T}}\right) \overline{\mathbf{W}}_{3}\left(\underline{\mathbf{G}}_{3} \underline{\mathbf{v}}+\underline{\mathbf{e}}_{3}\right) \\
& =\underline{\mathbf{v}}^{\mathrm{T}} \underbrace{\underline{\mathbf{v}}}_{\underline{\mathbf{W}}_{31}^{\mathbf{G}_{3}^{\mathrm{T}}} \overline{\mathbf{W}}_{3} \underline{\mathbf{G}}_{3}}+\underbrace{2 \underbrace{\mathrm{e}}_{3} \overline{\mathbf{W}}_{3} \underline{\mathbf{G}}_{3}}_{\underline{\mathbf{w}}_{32}^{\mathrm{T}}} \underline{\mathbf{v}}+\underbrace{\mathbf{e}_{3}^{\mathrm{T}} \overline{\mathbf{w}}_{3} \underline{\mathbf{e}}_{3}}_{w_{33}} \\
& =\underline{\mathbf{v}}^{\mathrm{T}} \underline{\mathbf{W}}_{31} \underline{\mathbf{v}}+\underline{\mathbf{w}}_{32}^{\mathrm{T}} \underline{\mathbf{v}}+w_{33}
\end{aligned}
$$

\section{Gesamtgüte}

Die Gesamtgüte für die Generierung der Quertrajektorie ergibt sich aus der Summe der Güteterme der einzelnen Segmente:

$$
\begin{aligned}
J_{\mathrm{Lat}}= & J_{1}+J_{2}+J_{3} \\
= & \underline{\mathbf{v}}^{\mathrm{T}} \underbrace{\left(\underline{\mathbf{w}}_{111}+\underline{\mathbf{w}}_{21}+\underline{\mathbf{w}}_{31}\right)}_{\underline{\mathbf{W}}_{\mathrm{G} 1}} \underline{\mathbf{v}}+\underbrace{\left(\underline{\mathbf{w}}_{112}^{\mathrm{T}}+\underline{\mathbf{w}}_{121}^{\mathrm{T}}+\underline{\mathbf{w}}_{22}^{\mathrm{T}}+\underline{\mathbf{w}}_{32}^{\mathrm{T}}\right)}_{w_{\mathrm{G} 3}} \underline{\mathbf{w}}_{\mathrm{G} 2}^{\mathrm{T}}+ \\
& \underbrace{\left(w_{113}+w_{122}+w_{131}+w_{23}+w_{33}\right)} \\
= & \underline{\mathbf{v}}^{\mathrm{T}} \underline{\mathbf{W}}_{\mathrm{G} 1} \underline{\mathbf{v}}+\underline{\mathbf{w}}_{\mathrm{G} 2}^{\mathrm{T}} \underline{\mathbf{v}}+w_{\mathrm{G} 3}
\end{aligned}
$$

Nach der Generierung von $\underline{\mathbf{W}}_{\mathrm{G} 1}, \underline{\mathbf{w}}_{\mathrm{G} 2}^{\mathrm{T}}$ und $w_{\mathrm{G} 3}$, basierend auf den Parametern $t_{1}, t_{2}, \underline{\mathbf{x}}_{0}, \underline{\mathbf{x}}_{1 \mathrm{~s}}$ und $\underline{\mathbf{x}}_{3 s}$, kann das quadratische Optimierungsproblem gelöst werden und somit das optimale $\underline{\mathbf{v}}$ bestimmt werden. Weitere Nebenbedingungen sind nicht erforderlich.

\section{A.2 Details zur Generierung des 3. Segmentes}

Für die Generierung des 3. Segmentes soll folgendes Gütemaß minimiert werden:

$$
J_{3}=\int_{0}^{\infty}\left[\underline{\mathbf{z}}(\tau)^{\mathrm{T}} \underline{\mathbf{W}} \underline{\mathbf{z}}(\tau)\right] \mathrm{d} \tau
$$

Die optimale Lösung wird durch einen Riccati-Regler erreicht. Hierzu wird die Größe $\underline{\mathbf{z}}$ in einen Zustand $\underline{\mathbf{v}}$ und einen skalaren Eingang $u$ aufgeteilt:

$$
\underline{\mathbf{z}}=\left[\begin{array}{l}
\underline{\mathbf{v}} \\
u
\end{array}\right]
$$

Der Zusammenhang zwischen $u$ und $\underline{\mathbf{v}}$ kann mit einer Integratorkette als System dargestellt werden:

$$
\underline{\mathbf{\mathbf { v }}}=\underbrace{\left[\begin{array}{lll}
0 & 1 & 0 \\
0 & 0 & 1 \\
0 & 0 & 0
\end{array}\right]}_{\underline{\mathbf{A}}} \underline{\mathbf{v}}+\underbrace{\left[\begin{array}{l}
0 \\
0 \\
1
\end{array}\right]}_{\underline{\mathbf{b}}} u
$$


Auch die Matrix $\underline{\mathbf{W}}$ wird in Einträge für Zustand und Eingang getrennt:

$$
\underline{\mathbf{W}}=\left[\begin{array}{ll}
\underline{\mathbf{Q}} & \underline{\mathbf{0}} \\
\mathbf{0} & r
\end{array}\right]
$$

Dadurch lässt sich $J_{3}$ wie folgt schreiben:

$$
\begin{aligned}
J_{3} & =\int_{0}^{\infty}\left[\underline{\mathbf{z}}^{\mathrm{T}} \underline{\mathbf{W}} \underline{\mathbf{z}}\right] \mathrm{d} \tau=\int_{0}^{\infty}\left[\begin{array}{ll}
\underline{\mathbf{v}}^{\mathrm{T}} & u
\end{array}\right]\left[\begin{array}{ll}
\underline{\mathbf{Q}} & \underline{\mathbf{0}} \\
\underline{\mathbf{0}} & r
\end{array}\right]\left[\begin{array}{l}
\underline{\mathbf{v}} \\
u
\end{array}\right] \mathrm{d} \tau \\
& =\int_{0}^{\infty}\left[\underline{\mathbf{v}}^{\mathrm{T}} \underline{\mathbf{Q}} \underline{\mathbf{v}}+u r u\right] \mathrm{d} \tau
\end{aligned}
$$

Mit einem Zustandsregler

$$
u=-\underline{\mathbf{k}}^{\mathrm{T}} \underline{\mathbf{v}}
$$

dessen Reglervektor $\underline{\mathbf{k}}$ sich aus der Lösung der Riccati-Gleichung ergibt, lässt sich $J_{3}$ minimieren [Föllinger, 2016, S. 339 ff.]. Damit ergibt sich das optimale Systemverhalten zu:

$$
\begin{aligned}
\dot{\mathbf{\mathbf { v }}} & =\underbrace{\left[\underline{\mathbf{A}}-\underline{\mathbf{b}}^{\mathrm{T}}\right]}_{\underline{\mathbf{A}}_{K}} \cdot \underline{\mathbf{v}} \\
\Rightarrow \underline{\mathbf{v}}(\tau) & =\mathrm{e}^{\mathrm{A}_{\mathrm{k}} \tau} \underline{\mathbf{v}}_{0}
\end{aligned}
$$

Setzt man den Regler in Gleichung (A.39) ein, so erhält man:

$$
\begin{aligned}
J_{3} & =\int_{0}^{\infty}\left[\underline{\mathbf{v}}^{\mathrm{T}} \underline{\mathbf{Q}} \underline{\mathbf{v}}+\underline{\mathbf{v}}^{\mathrm{T}} \underline{\mathbf{k}} r \underline{\mathbf{k}}^{\mathrm{T}} \underline{\mathbf{v}}\right] \mathrm{d} \tau=\int_{0}^{\infty}\left[\underline{\mathbf{v}}^{\mathrm{T}}\left(\underline{\mathbf{Q}}+\underline{\mathbf{k}} r \underline{\mathbf{k}}^{\mathrm{T}}\right) \underline{\mathbf{v}}\right] \mathrm{d} \tau \\
= & \underbrace{\int_{0}^{\infty}[\mathrm{e}^{\mathrm{A}^{\mathrm{A}} \tau} \underbrace{\left(\underline{\mathbf{Q}}+\underline{\mathbf{k}} r \underline{\mathbf{k}}^{\mathrm{T}}\right)}_{\hat{\mathbf{W}}} \mathrm{e}^{\left.\underline{\mathbf{A}}_{\mathrm{k}} \tau\right]} \mathrm{d} \tau}_{\tilde{\mathbf{v}} 0} \cdot \underline{\mathbf{v}}_{0}
\end{aligned}
$$

Das Integral ist unabhängig von $\underline{\mathbf{v}}_{0}$ und somit lässt es sich für ein gegebenes System offline als Lösung der Lyapunov-Gleichung $\underline{A}_{k}^{\mathrm{T}} \underline{\tilde{\mathbf{W}}}+\underline{\mathbf{W}} \mathbf{A}_{k}=-\underline{\mathbf{W}}$ berechnen. Wird das berechnete Integral mit $\underline{\tilde{W}}$ bezeichnet, so ergibt sich:

$$
J_{3}=\underline{\mathbf{v}}_{0}^{\mathrm{T}} \cdot \underline{\tilde{\mathbf{w}}} \cdot \underline{\mathbf{v}}_{0}
$$

$\underline{\mathbf{v}}_{0}$ ergibt sich aus $\underline{\mathbf{z}}_{0}$ durch:

$$
\underline{\mathbf{v}}_{0}=\underbrace{\left[\begin{array}{llll}
1 & 0 & 0 & 0 \\
0 & 1 & 0 & 0 \\
0 & 0 & 1 & 0
\end{array}\right]}_{\underline{\mathbf{C}}_{2}} \underline{\mathbf{z}}_{0}
$$


und somit $J_{3} \mathrm{zu}$ :

$$
\begin{aligned}
& J_{3}=\underline{\mathbf{z}}_{0}^{\mathrm{T}} \cdot \underbrace{\underline{\mathbf{C}}_{2}^{\mathrm{T}} \int_{0}^{\infty}\left[\mathrm{e}^{\mathbf{A}^{\mathrm{T}} \tau}\left(\underline{\mathbf{Q}}+\underline{\mathbf{k}} r \underline{\mathbf{k}}^{\mathrm{T}}\right) \mathrm{e}^{\mathbf{A}_{\mathbf{k}} \tau}\right] \mathrm{d} \tau \cdot \underline{\mathbf{C}}_{2}}_{\underline{\mathbf{W}}_{3}} \cdot \underline{\mathbf{z}}_{0} \\
& =\underline{\mathbf{z}}_{0}^{\mathrm{T}} \cdot \underbrace{\underline{\mathbf{C}}_{2}^{\mathrm{T}} \underline{\tilde{\mathbf{w}}} \underline{\mathbf{C}}_{2}}_{\underline{\mathbf{W}}_{3}} \underline{\mathbf{z}}_{0} \\
& =\underline{\mathbf{z}}_{0}^{\mathrm{T}} \overline{\mathbf{W}}_{3} \underline{\mathbf{z}}_{0}
\end{aligned}
$$

In der Implementierung wurden der Regler $\underline{\mathbf{k}}$ sowie die Matrix $\underline{\mathbf{W}}_{3}$ mit Hilfe von MatLAB, der Control-System-Toolbox sowie der Symbolic-Math-Toolbox bestimmt. 
Die Quertrajektorie lässt sich über die Diagonaleinträge der Matrizen $\underline{\mathbf{W}}_{1}, \underline{\mathbf{W}}_{2}$ und $\underline{\mathbf{W}}_{3}$ parametrieren. Auf diese Weise ist es beispielsweise möglich, den Fahrstreifenwechsel etwas früher oder später beginnen zu lassen. Für ein paar beispielhafte Parametersätze sind die Quertrajektorien in Abbildung A.3 dargestellt. Die Fahrstreifenbreite beträgt dabei 3,5 m.

- A: Erste Parametrierung, starke Gewichtung der Segmente 1 und 3.

\begin{tabular}{l|cccc} 
& $w_{\mathrm{p}}$ & $w_{\mathrm{v}}$ & $w_{\mathrm{a}}$ & $w_{\mathrm{j}}$ \\
\hline Seg. 1 & 100 & 200 & 100 & 1 \\
Seg. 2 & 0 & 0 & 0 & 1 \\
Seg. 3 & 100 & 200 & 100 & 1
\end{tabular}

- B: Zweite Parametrierung, gegenüber A reduzierte Bestrafung der Nicht-Ruck-Größen.

\begin{tabular}{c|cccc} 
& $w_{\mathrm{p}}$ & $w_{\mathrm{v}}$ & $w_{\mathrm{a}}$ & $w_{\mathrm{j}}$ \\
\hline Seg. 1 & 4 & 2 & 1 & 1 \\
Seg. 2 & 0 & 0 & 0 & 1 \\
Seg. 3 & 4 & 2 & 1 & 1
\end{tabular}

- C: Finale Parametrierung, gegenüber B reduzierte Gewichtung des ersten Segmentes im Vergleich zum dritten.

\begin{tabular}{c|cccc} 
& $w_{\mathrm{p}}$ & $w_{\mathrm{v}}$ & $w_{\mathrm{a}}$ & $w_{\mathrm{j}}$ \\
\hline Seg. 1 & 1 & 2 & 1 & 1 \\
Seg. 2 & 0 & 0 & 0 & 1 \\
Seg. 3 & 10 & 2 & 1 & 1
\end{tabular}

- D: Alternativer Parametersatz, der zu einem früheren Beginn des Fahrstreifenwechsels führt.

\begin{tabular}{c|cccc} 
& $w_{\mathrm{p}}$ & $w_{\mathrm{v}}$ & $w_{\mathrm{a}}$ & $w_{\mathrm{j}}$ \\
\hline Seg. 1 & 4 & 2 & 1 & 1 \\
Seg. 2 & 0 & 2 & 1 & 1 \\
Seg. 3 & 10 & 2 & 1 & 1
\end{tabular}




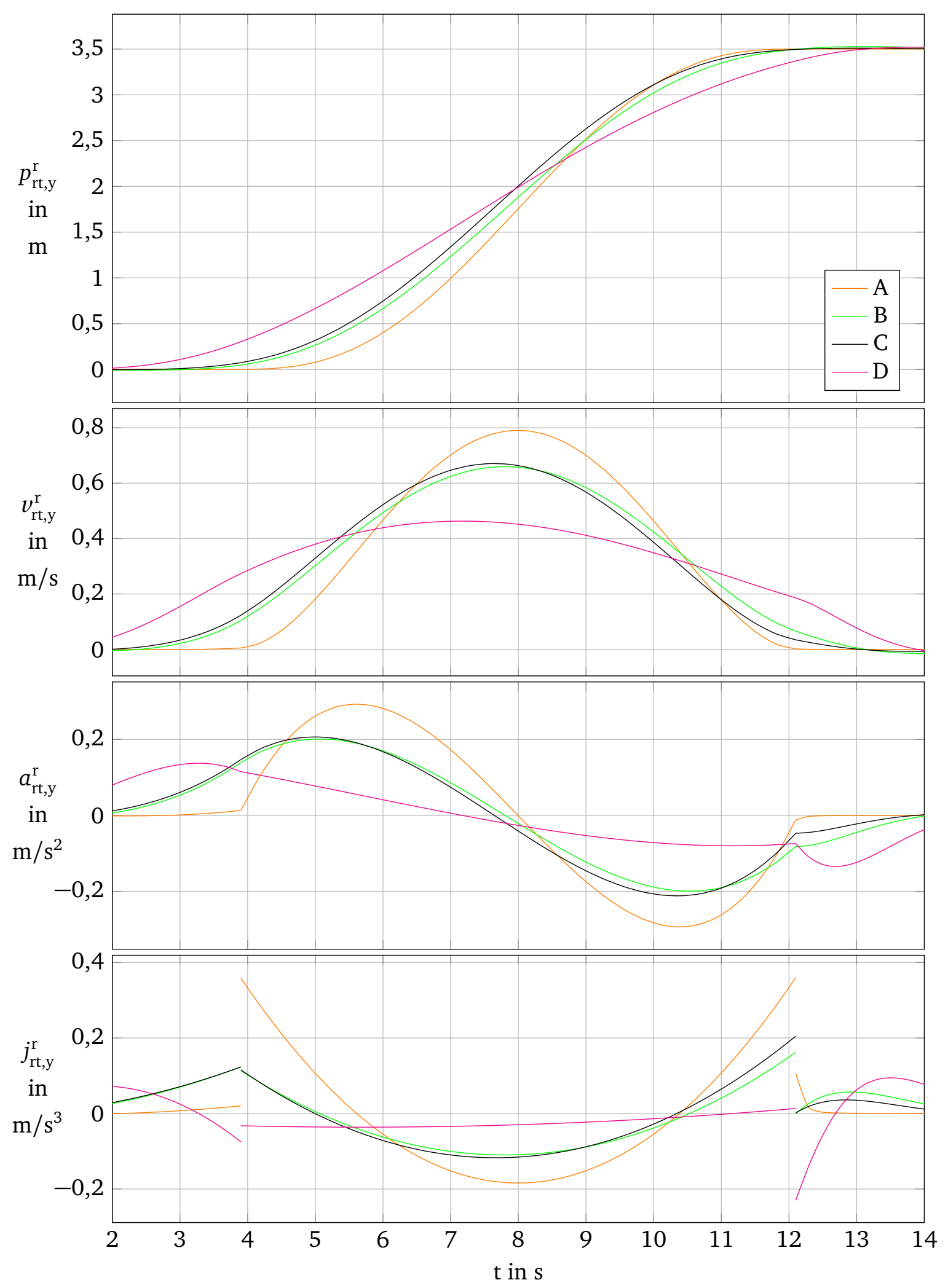

Abbildung A.3: Vergleich unterschiedlicher Parametersätze für Quertrajektorien 
Würde man nur eine ruckoptimale Trajektorie ohne Beschränkung von $t_{2}$ suchen, wäre das Ergebnis ein unendlich lange dauernder Fahrstreifenwechsel. Da dies jedoch nicht dem menschlichen Fahrverhalten entspricht, liegt es nahe, die Dauer des Fahrstreifenwechsels zu begrenzen. Durch die zyklische Neuplanung ist es nicht sinnvoll, dafür eine Zeitdauer fest vorzugeben. Vielmehr wird die maximale Restdauer des Fahrstreifenwechsels abhängig vom aktuellen Planungs-Startzustand berechnet. Dies geschieht durch einen zusätzlichen Strafterm in der Güte der Quertrajektorie:

$$
\tilde{J}_{\text {Lat }}=\int_{0}^{t_{1}} \ldots \mathrm{d} t+\int_{t_{1}}^{t_{2}} \ldots \mathrm{d} t+\int_{t_{2}}^{\infty} \ldots \mathrm{d} t+\underbrace{W_{\Delta t_{1} t_{2}} \cdot(\overbrace{t_{2}-t_{1}}^{\Delta t})}_{\text {Strafterm }}
$$

Aufgabe ist es nun, das $t_{2 \text {, max }}$ zu bestimmen, das $\tilde{J}_{\text {Lat }}$ minimiert. Dies geschieht mit Hilfe des Downhill-Simplex-Verfahrens [Nelder, 1965]. Ein beispielhafter Verlauf von $\tilde{J}_{\text {Lat }}\left(t_{2}\right)$ ist in Abbildung A.4 dargestellt.

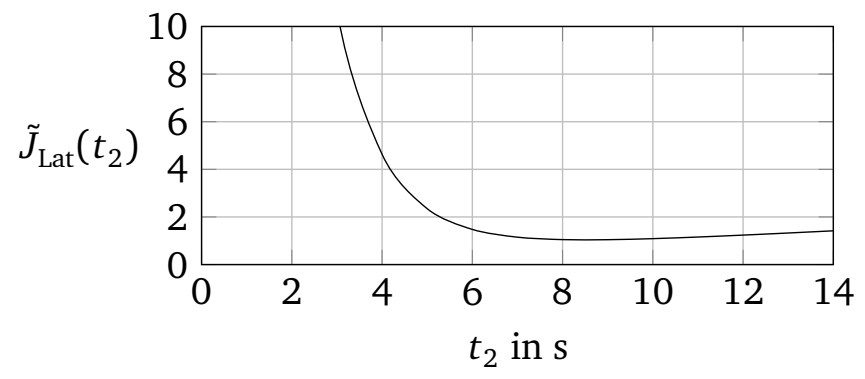

Abbildung A.4: Beispiel für den Verlauf des Gütemaßes $\tilde{J}_{\text {Lat }}$ in Abhängigkeit von $t_{2}$

Die sich bei der Optimierung ergebende Fahrstreifenwechseldauer $t_{2}-t_{1}$ wird mit $\Delta t_{\max }$ bezeichnet. Bei der Bestimmung von $\Delta t_{\max }$ müssen zwei Fälle unterschieden werden:

\section{Planung von 3 Segmenten}

Da für jeden Trajektorienkandidaten ein anderes $t_{1}$ gilt, müsste theoretisch für jeden Kandidaten $\Delta t_{\max }$ neu bestimmt werden. Der dabei entstehende Berechnungsaufwand steht in keinem Verhältnis zum erzielten Nutzen.

Aus diesem Grund wird an dieser Stelle eine Vereinfachung durchgeführt. In Situationen, in denen $\Delta t_{\text {max }}$ relevant wird, tritt im 1. Segment nur eine sehr geringe Bewegung auf. Daher wird dieser Term für die Berechnung vernachlässigt und $t_{1}=0$ gesetzt. Als Startzustand für die Quertrajektorie wird die Mitte des Start-Fahrstreifens verwendet (Abbildung A.5). Auf diese Weise muss zur Planung einer Trajektorie nur einmal $\Delta t_{\max }$ bestimmt werden. 


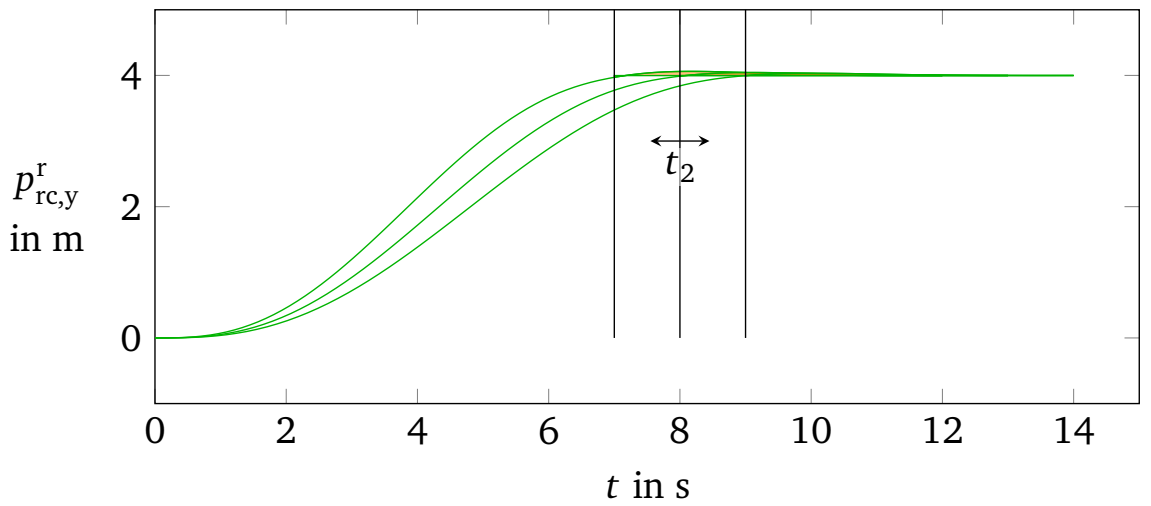

Ego-Traj.

Abbildung A.5: Beispielhafte Quertrajektorie mit variablem $t_{2}$ zur Bestimmung von $\Delta t_{\max }$ bei der Planung einer Trajektorie mit 3 Segmenten

\section{Planung von 2 Segmenten}

Da das erste Segment nicht vorhanden ist, sind keine Vereinfachungen notwendig. Der Planungs-Startzustand kann direkt als Anfangsbedingung für die Trajektorie übernommen werden (Abbildung A.6).

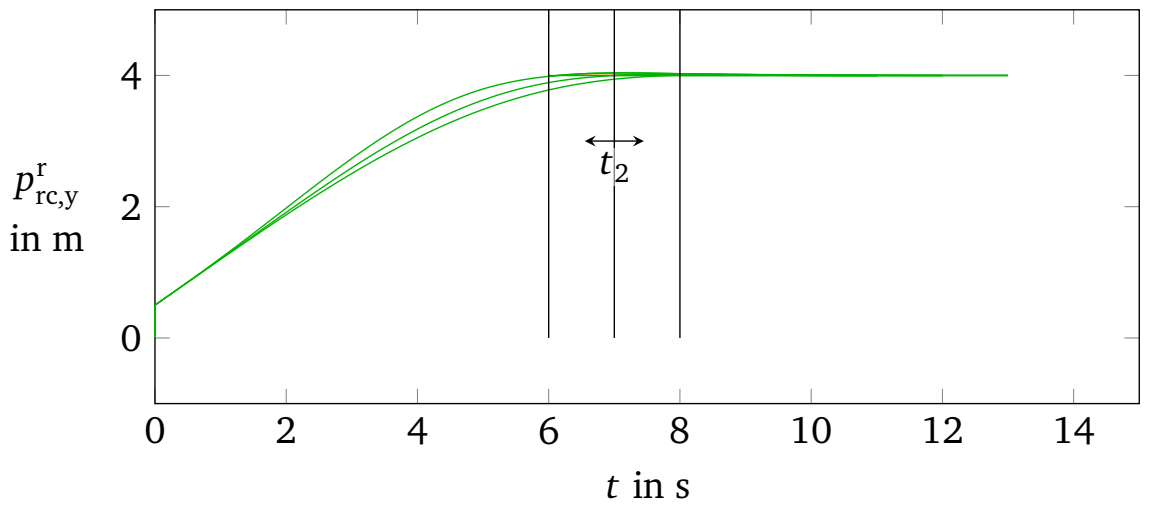

Ego-Traj.

Abbildung A.6: Beispielhafte Quertrajektorie mit variablem $t_{2}$ zur Bestimmung von $\Delta t_{\max }$ bei der Planung einer Trajektorie mit 2 Segmenten

\section{A.5 Begrenzung von $\bar{v}_{1}$ und $\bar{v}_{2}$ zur Einschränkung der Startwerte}

Teilweise ist es bei Fahrversuchen dazu gekommen, dass aufgrund von ungünstigen oder ungültigen Startwerten für $\bar{v}_{1}$ der Downhill-Simplex-Algorithmus keine Lösung gefunden hat. Um dies zu vermeiden, wird $\bar{v}_{1}$ so begrenzt, dass die Startparameter zu einer gültigen Trajektorie führen. Der Algorithmus muss dabei mit allen Umfeldfahrzeugkombinationen funktionieren. Der Ansatz ist dabei, dass ein fiktives Fahrzeug, das sich mit $\bar{v}_{1}$ bewegt, die Wechsellücke erreichen muss.

Hierzu wird folgender Algorithmus verwendet:

Neben den Umfeldfahrzeugen wird auch die minimal und maximal mögliche Geschwindigkeit 
des Fahrzeugs $\left(v_{\min }=0, v_{\max }=v_{\mathrm{ACC}, \text { Setz }}\right)$ sowie eine Endzeit $t_{\max }$ in das $x(t)$-Diagramm eingetragen (Abbildung A.7) (In den Fahrversuchen: $t_{\max }=25 \mathrm{~s}$ ).

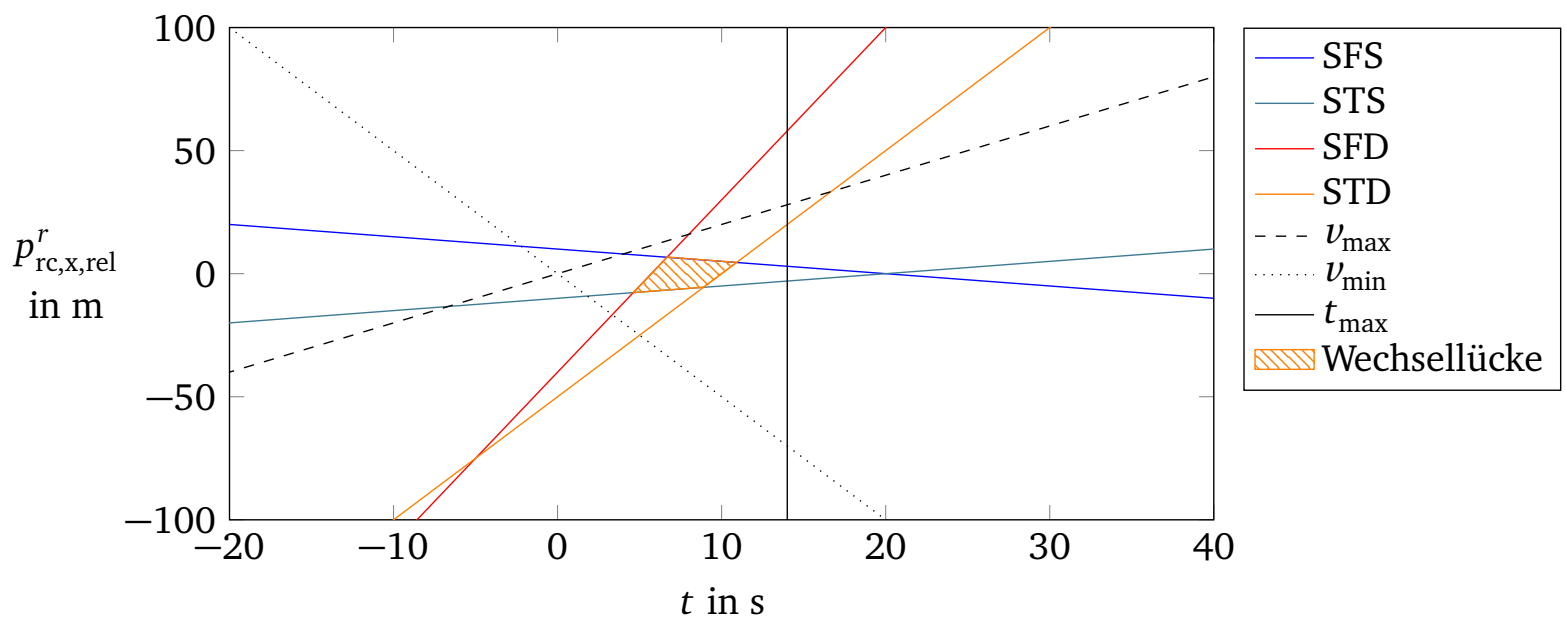

Abbildung A.7: Umfeld inkl. minimaler sowie maximaler Ego-Geschwindigkeit und Endzeit

Es entstehen 7 Geraden in der Zeichnung. Daher gibt es maximal 21 Schnittpunkte:

$$
n_{\text {Schnittpunkte }}=\sum_{i=1}^{n_{\text {Geraden }}-1} i=\frac{\left(n_{\text {Geraden }}-1\right) \cdot\left(n_{\text {Geraden }}\right)}{2}
$$

(siehe auch [Bronstein, 2001, S.19]). Es werden nun systematisch die Schnittpunkte der Geraden berechnet. Ist ein Fahrzeug nicht vorhanden oder schneiden sich zwei Geraden nicht, so wird NaN als Schnittpunkt ausgegeben (Abbildung A.8).

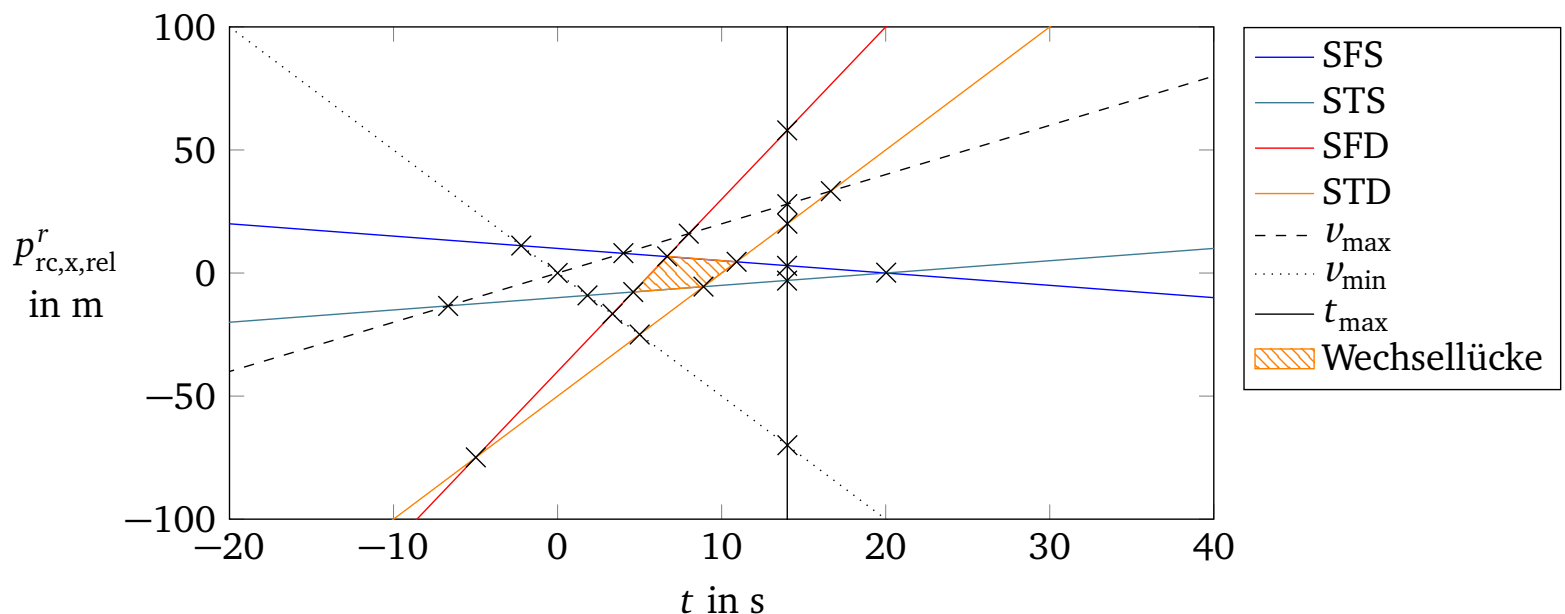

Abbildung A.8: Umfeld inkl. minimaler sowie maximaler Ego-Geschwindigkeit, Endzeit und den Schnittpunkten

Im Weiteren wird bestimmt, welche der Schnittpunkte sich

- außerhalb der Wechsellücke 
- oberhalb der $v_{\max }$ Geraden

- unterhalb der $v_{\min }$ Geraden

- hinter der $t_{\max }$ Geraden

befinden. Alle diese Schnittpunkte werden ebenfalls mit NaN überschrieben (Abbildung A.9).

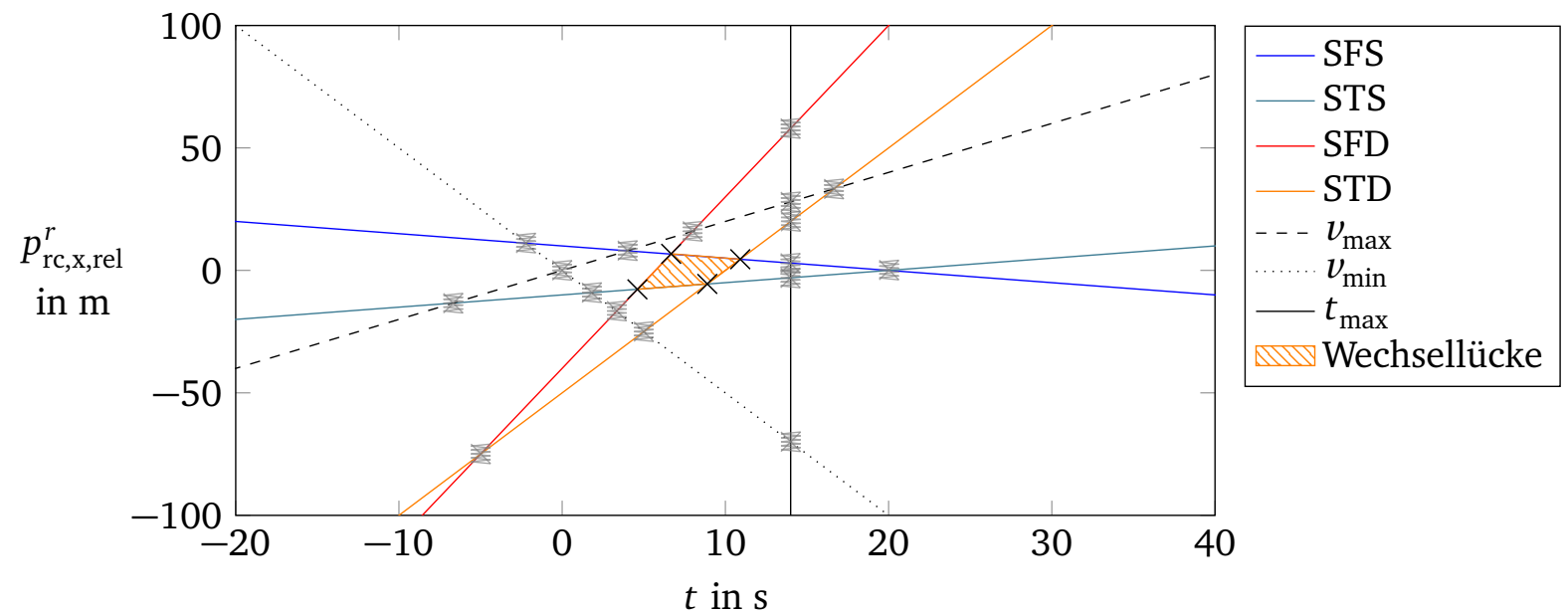

Abbildung A.9: Umfeld inkl. minimaler sowie maximaler Ego-Geschwindigkeit, Endzeit und den Schnittpunkten am Rand der Wechsellücke

$\mathrm{Zu}$ den verbleibenden Schnittpunkten werden die Durchschnittsgeschwindigkeiten $\bar{v}_{1, i}$ berechnet, die notwendig sind, um die Punkte zu erreichen (Abbildung A.10).

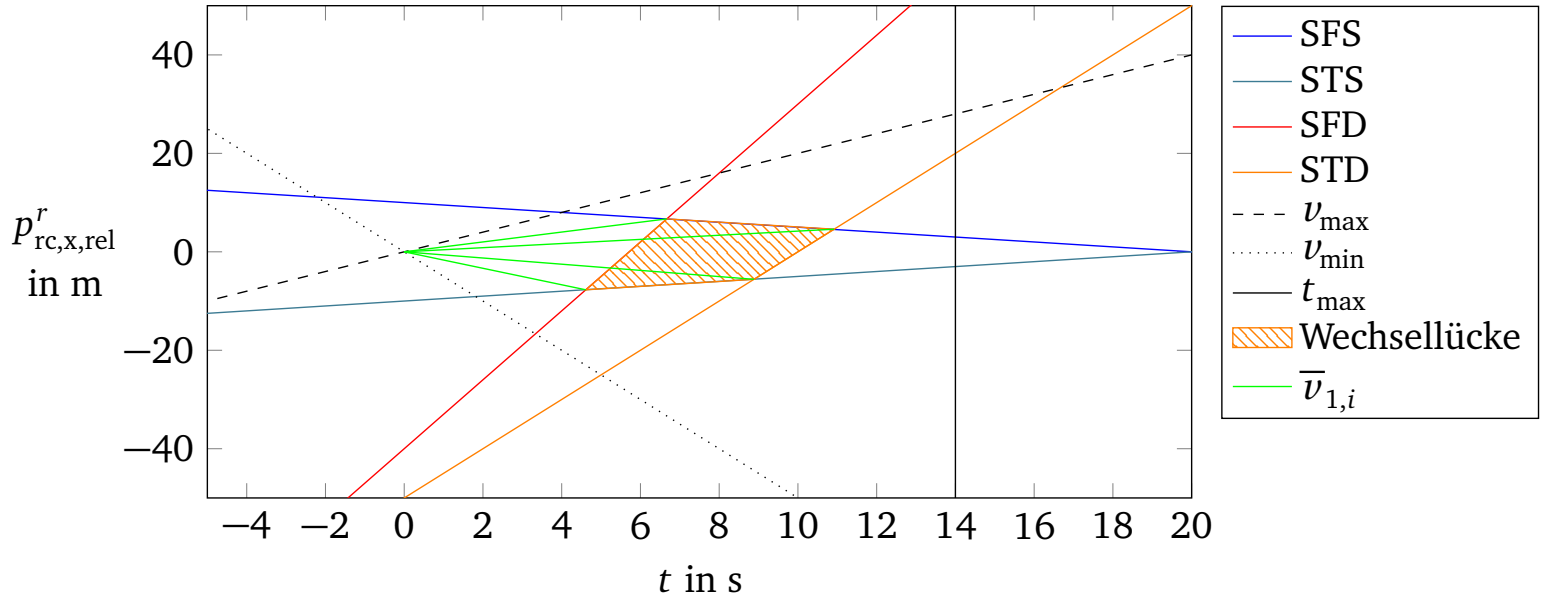

Abbildung A.10: Umfeld inkl. minimaler sowie maximaler Ego-Geschwindigkeit, Endzeit und den Durchschnittsgeschwindigkeiten

Maximum und Minimum für $\bar{v}_{1}$ ergeben sich nun aus dem minimalen und maximalen $\bar{v}_{1, i}$ und einer kleinen Toleranz, die numerische Probleme vermeidet:

$$
\bar{v}_{1, \max }=\max _{i}\left(\bar{v}_{1, i}\right)-0,01 \mathrm{~km} / \mathrm{h} \quad \bar{v}_{1, \min }=\min _{i}\left(\bar{v}_{1, i}\right)+0,01 \mathrm{~km} / \mathrm{h}
$$


Nachdem ein gültiger Startwert für $\bar{v}_{1}$ gefunden wurde, wird ein ähnliches, aber einfacheres Verfahren auch für $\bar{v}_{2}$ angewendet. Hierbei wird das minimale und maximale $\bar{v}_{2}$ bestimmt, das dazu führt, dass sich das Fahrzeug $1 \mathrm{~ms}$ nach $t_{1}$ noch innerhalb der Lücke befindet. Falls sich dieser Zeitpunkt schon außerhalb der Wechsellücke befindet, wird der Startwert von $\bar{v}_{2}$ nicht beschränkt.

\section{A.6 Die unterschiedlichen Startzustände der Planung (Fallunterscheidungen)}

Je nach Situation besitzt die zu planende Trajektorie eine unterschiedliche Anzahl von Segmenten. Hierfür sind daher auch unterschiedliche Planungsfunktionen notwendig. Eine Trajektorie besitzt entweder ein, zwei oder drei Segmente (Tabelle A.1).

Tabelle A.1: Übersicht über die Trajektorien und deren Segmente

\begin{tabular}{c|ccc|ccc} 
Anzahl & \multicolumn{3}{|c|}{ Längs } & \multicolumn{3}{|c}{ Quer } \\
Segmente & Seg. 1 & Seg. 2 & Seg. 3 & Seg. 1 & Seg. 2 & Seg. 3 \\
\hline 1 & - & - & ACC & - & - & Exp. \\
2 & - & Polyn. & ACC & - & Polyn. & Exp. \\
3 & Polyn. & Polyn. & ACC & Polyn. & Polyn. & Exp.
\end{tabular}

Je nach Startzustand müssen unterschiedlich viele Segmente geplant werden. Zunächst wird bestimmt, welche Fahrstreifen das Fahrzeug im Startzustand belegt. Ein Fahrstreifen gilt als belegt, wenn sich ein Teil des Fahrzeugs auf ihm befindet. Aktuell ist eine Planung nur aus bestimmten Situationen heraus möglich. Dies betrifft insbesondere die Vermeidung der Sicherheitsbereiche.

\section{Nur der Start-Fahrstreifen wird belegt}

Hier wird gefordert, dass die Sicherheitsbereiche zu FD und TD eingehalten werden. Ist dies nicht der Fall, ist eine Planung nicht möglich. Befindet sich das eigene Fahrzeug schon auf der Höhe des befahrbaren Bereiches auf dem Ziel-Fahrstreifen, so wird eine Trajektorie mit 2 Segmenten geplant. Andernfalls werden 3 Segmente geplant.
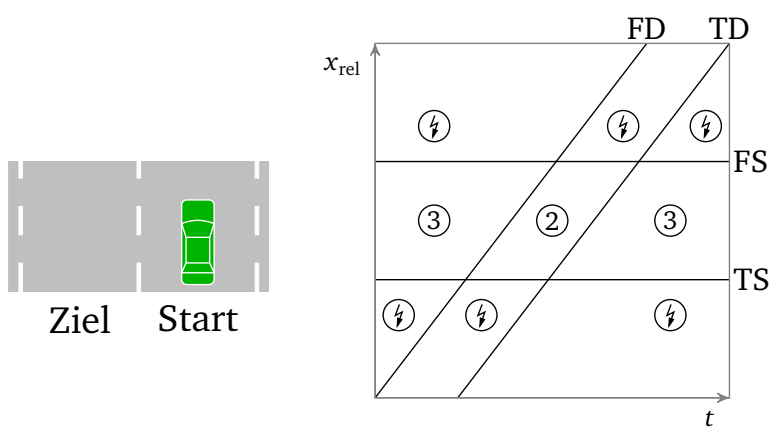

Abbildung A.11: Anzahl der Segmente, wenn nur der Start-Fahrstreifen belegt wird 


\section{Start- und Zielfahrstreifen werden belegt}

Befindet sich das eigene Fahrzeug in der Wechsellücke, wird eine Trajektorie mit 2 Segmenten geplant.

Wenn das eigene Fahrzeug den Sicherheitsbereich des Start-Fahrstreifens verletzt, wäre normalerweise keine Trajektorie planbar. Sofern der Zielfahrstreifen befahrbar ist, wird eine Trajektorie mit einem Segment geplant, um in diesem Fall trotzdem eine Planung zu ermöglichen. In dieser Situation würde das eigene Fahrzeug möglichst schnell auf den Zielfahrstreifen wechseln.

In Fällen, in denen sich das eigene Fahrzeug in den Sicherheitsbereichen der Fahrzeuge auf dem Zielfahrstreifen befindet (hier mit $*$ markiert), findet eine weitere Fallunterscheidung statt. Sofern sich das eigene Fahrzeug mit seinem Koordinatenursprung schon auf dem Zielfahrstreifen befindet, ist eine Planung nicht möglich. Andernfalls wird eine Trajektorie mit 3 Segmenten geplant. Diese zögert den Fahrstreifenwechsel noch etwas heraus und wechselt anschließend.
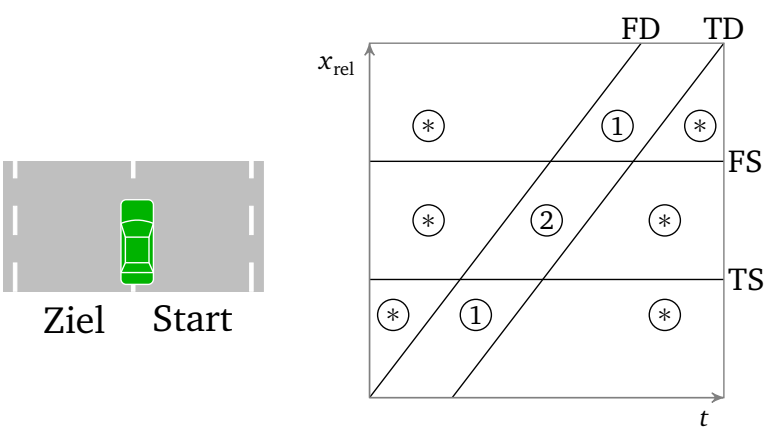

Abbildung A.12: Anzahl der Segmente, wenn beide Fahrstreifen belegt werden

\section{Nur der Zielfahrstreifen wird belegt}

Wenn das eigene Fahrzeug die Sicherheitsbereiche von FD und TD verletzt, ist eine Planung nicht möglich. Ist das eigene Fahrzeug noch in der Wechsellücke, so wird eine Trajektorie mit 2 Segmenten berechnet, andernfalls wird eine Trajektorie mit einem Segment berechnet.
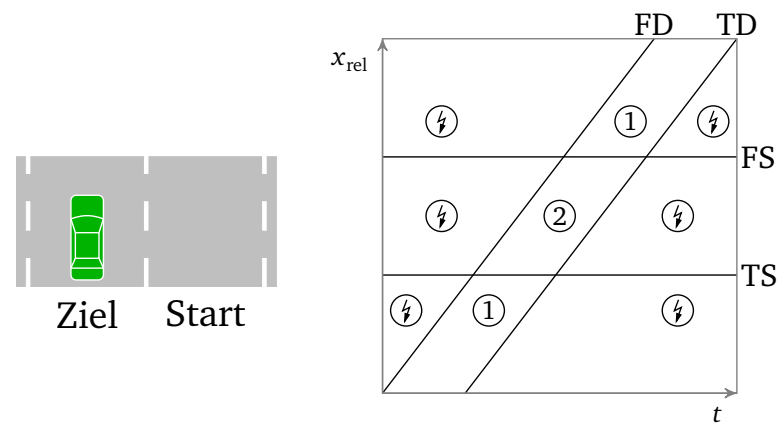

Abbildung A.13: Anzahl der Segmente, wenn nur der Ziel-Fahrstreifen belegt wird 
Für einige der Trajektorienbewertungen wird die Trajektorie abgetastet. Unter der Annahme einer Maximalbeschleunigung $a_{\max }$ des Fahrzeugs lässt sich ausrechnen, wie groß die maximale Abweichung in der Fahrzeugposition zwischen den Abtastpunkten ist. Hierbei wird davon ausgegangen, dass die Trajektorie mit einer Abtastzeit $T$ abgetastet wird (Abbildung A.14).

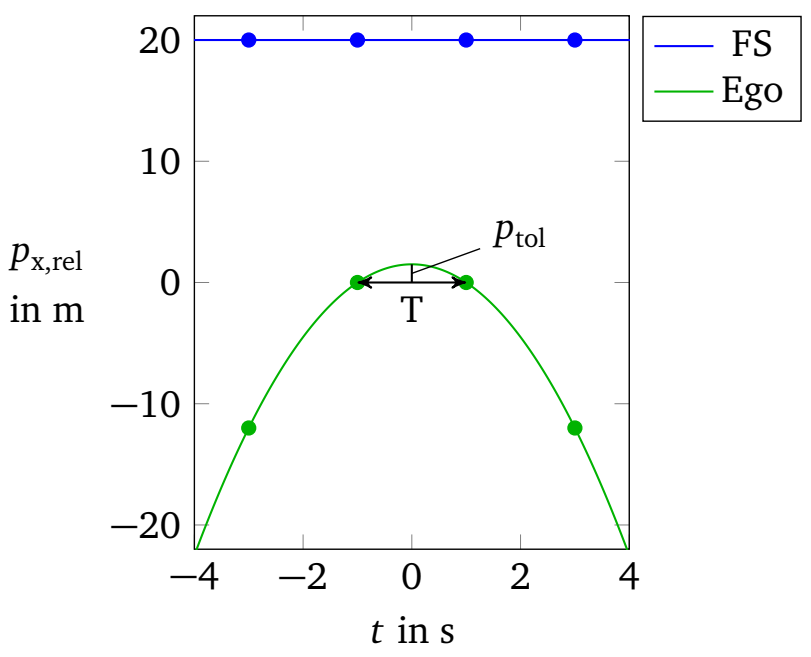

Abbildung A.14: Abtastung der Trajektorie

Die beiden betrachteten Abtastzeitpunkte halten den Mindestabstand zum vorausfahrenden Fahrzeug ein. Relevant ist die Frage: Wie weit kann sich die Trajektorie dem vorderen Fahrzeug zwischen diesen beiden Abtastzeitpunkten annähern? Bei einer bekannten maximal möglichen Beschleunigung $a_{\max }$ tritt maximal eine Toleranz von

$$
p_{\text {tol }}=\frac{1}{2} a_{\max }\left(\frac{T}{2}\right)^{2}
$$

auf. Für eine maximale Beschleunigung von $a_{\max }=4 \mathrm{~m} / \mathrm{s}^{2}$ und einer Abtastzeit von $T=1 \mathrm{~s}$ ergibt sich $p_{\text {tol }}=0,5 \mathrm{~m}$ und bei $T=0,5 \mathrm{~s}$ sogar nur $p_{\text {tol }}=0,125 \mathrm{~m}$. Diese Toleranz ist bei den Sicherheitsabständen im Bereich von einigen Metern akzeptabel. 


\section{B Detailbetrachtungen der Spline-Planung}

\section{B.1 Generierung der Spline-Trajektorie}

Verwendete Symbole:

\begin{tabular}{ll} 
Symbol & Beschreibung \\
\hline$n$ & Anzahl Segmente \\
$T$ & Zeitliche Dauer eines Segmentes \\
$\underline{\mathbf{p}}_{i}=\left[\begin{array}{llll}b_{i} & c_{i} & d_{i} & e_{i}\end{array}\right]^{\mathrm{T}}$ & $\begin{array}{l}\text { Polynomkoeffizienten des } i \text {-ten Segmentes } \\
j\end{array}$
\end{tabular}

Wie in Abschnitt 5.2 beschrieben, besteht die Spline-Trajektorie in Längs- und Querrichtung aus je $n=15$ Segmenten. Diese Segmente werden jeweils durch Polynome dritten Grades beschrieben. Daraus ergeben sich insgesamt $4 \cdot 15 \cdot 2=120$ Polynomkoeffizienten. Um nicht mit 120 Optimierungsvariablen zu arbeiten, werden die Nebenbedingungen ausgenutzt, um einen Teil davon zu eliminieren.

Der Spline soll stetig in den Größen Position $p$, Geschwindigkeit $v$ sowie der Beschleunigung a sein. Drei der vier Koeffizienten jedes Segmentes ergeben sich daher aus den Stetigkeitsbedingungen. Auf diese Weise lässt sich die Anzahl der Optimierungsvariablen von 120 auf 30 reduzieren (15 Längs, 15 Quer). In Abbildung B.1 ist beispielhaft der Verlauf der einzelnen Zustandsgrößen bei einem Spline mit 5 Segmenten gezeigt. Wie die Stetigkeitsbedingungen eingesetzt werden und welche Rechenzeitoptimierungen bei der Berechnung der Gütemaße vorgenommen werden, wird im Folgenden gezeigt.
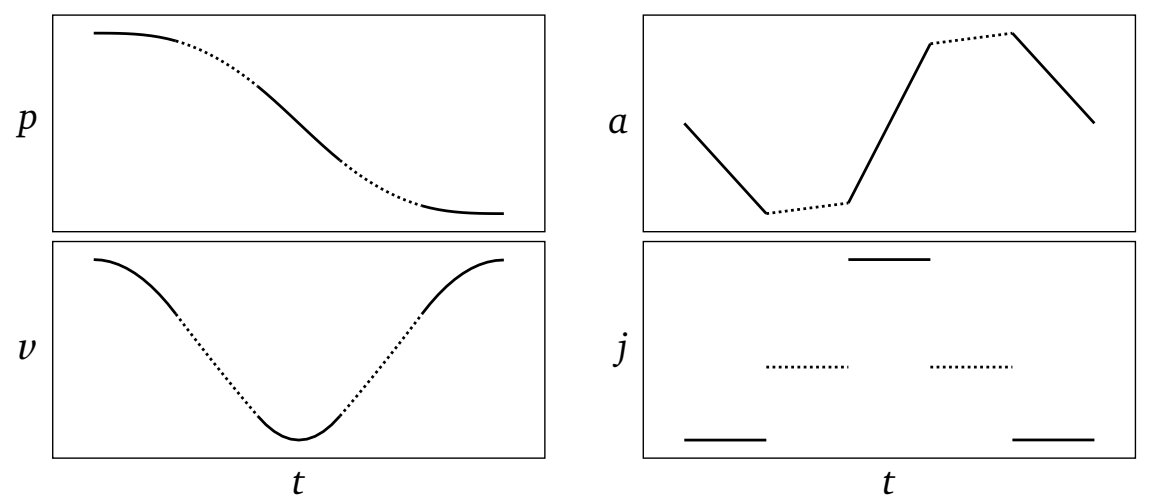

Abbildung B.1: Ein Spline mit 5 Segmenten aus Polynomen 3. Grades zusammen mit den ersten drei Ableitungen 
In den Fahrversuchen wurde für jedes Spline-Segment eine feste Länge von $T=1 \mathrm{~s}$ gewählt. Damit ergibt sich folgende abschnittsweise Definition, bei der jedes Segment von einer eigenen Funktion beschrieben wird:

$$
\underline{\mathbf{f}}(t)= \begin{cases}\underline{\mathbf{f}}_{1}(t) & 0 \leq t \leq T \\ \underline{\mathbf{f}}_{2}(t-T) & T \leq t \leq 2 T \\ \vdots & \vdots \\ \underline{\mathbf{f}}_{n}(t-(n-1) T) & (n-1) T \leq t \leq n T\end{cases}
$$

Die Vektorfunktion $\underline{\mathbf{f}}_{i}(\tau)$ fasst dabei den Wert und die Ableitungen bis zur Ordnung 3 des Splines zusammen:

$$
\underline{\mathbf{f}}_{i}(\tau)=\left[\begin{array}{c}
p_{i} \\
v_{i} \\
a_{i} \\
j_{i}
\end{array}\right]=\left[\begin{array}{c}
f_{i}(\tau) \\
f_{i}^{\prime}(\tau) \\
f_{i}^{\prime \prime}(\tau) \\
f_{i}^{\prime \prime \prime}(\tau)
\end{array}\right]=\left[\begin{array}{rrrrrrrrrr}
b_{i} & + & c_{i} & \tau & + & d_{i} & \tau^{2} & + & e_{i} & \tau^{3} \\
& c_{i} & & + & 2 d_{i} & \tau & + & 3 e_{i} & \tau^{2} \\
& & & & 2 d_{i} & & + & 6 e_{i} & \tau \\
& & & & & & & & & \\
& & & & & & & &
\end{array}\right]
$$

Dies lässt sich kompakt in Matrixform darstellen, bei der die Polynomkoeffizienten im Vektor $\underline{\mathbf{p}}_{i}$ enthalten sind:

$$
\underline{\mathbf{f}}_{i}(\tau)=\left[\begin{array}{cccc}
1 & \tau & \tau^{2} & \tau^{3} \\
0 & 1 & 2 \tau & 3 \tau^{2} \\
0 & 0 & 2 & 6 \tau \\
0 & 0 & 0 & 6
\end{array}\right]\left[\begin{array}{c}
b_{i} \\
c_{i} \\
d_{i} \\
e_{i}
\end{array}\right]=\underline{\mathbf{F}}_{\mathrm{b}}^{\mathrm{T}}(\tau) \cdot \underline{\mathbf{p}}_{i}
$$

Am Anfangs- und am Endpunkt jedes Segmentes ergeben sich für $\underline{\mathbf{F}}_{\mathrm{b}}^{\mathrm{T}}(\tau)$ folgende Werte:

$$
\underline{\mathbf{F}}_{\mathrm{b}}^{\mathrm{T}}(0)=\left[\begin{array}{cccc}
1 & 0 & 0 & 0 \\
0 & 1 & 0 & 0 \\
0 & 0 & 2 & 0 \\
0 & 0 & 0 & 6
\end{array}\right] \quad \underline{\mathbf{F}}_{\mathrm{b}}^{\mathrm{T}}(T)=\left[\begin{array}{cccc}
1 & T & T^{2} & T^{3} \\
0 & 1 & 2 T & 3 T^{2} \\
0 & 0 & 2 & 6 T \\
0 & 0 & 0 & 6
\end{array}\right]
$$


Bei Berücksichtigung der Anfangsbedingung $\underline{\mathbf{x}}_{0}=\left[\begin{array}{lll}p_{0} & v_{0} & a_{0}\end{array}\right]^{\mathrm{T}}$ für das erste Segment ergibt sich:

$$
\begin{aligned}
\underline{\mathbf{F}}_{\mathrm{b}}^{\mathrm{T}}(0) \cdot \underline{\mathbf{p}}_{1} & =\left[\begin{array}{c}
\underline{\mathbf{x}}_{0} \\
j_{1}
\end{array}\right] \\
\underline{\mathbf{p}}_{1} & =\left(\underline{\mathbf{F}}_{b}^{\mathrm{T}}(0)\right)^{-1}\left[\begin{array}{c}
\underline{\mathbf{x}}_{0} \\
j_{1}
\end{array}\right] \\
\underline{\mathbf{p}}_{1} & =\left[\begin{array}{llll}
1 & 0 & 0 & 0 \\
0 & 1 & 0 & 0 \\
0 & 0 & \frac{1}{2} & 0 \\
0 & 0 & 0 & \frac{1}{6}
\end{array}\right]\left[\begin{array}{c}
\underline{\mathbf{x}}_{0} \\
j_{1}
\end{array}\right] \\
\underline{\mathbf{p}}_{1} & =\underbrace{\left[\begin{array}{lll}
1 & 0 & 0 \\
0 & 1 & 0 \\
0 & 0 & \frac{1}{2} \\
0 & 0 & 0
\end{array}\right]}_{\underline{\mathbf{A}}} \cdot \underbrace{\left[\begin{array}{c}
0 \\
0 \\
0 \\
1 \\
\frac{1}{6}
\end{array}\right]}_{0} \cdot j_{1} \\
\underline{\mathbf{p}}_{1} & =\underline{\mathbf{\mathbf { a }} \cdot \underline{\mathbf{x}}_{0}+\underline{\mathbf{b}} \cdot j_{1}}
\end{aligned}
$$

Das Spline-Segment 1 wird also durch die Anfangsbedingung $\underline{\mathbf{x}}_{0}$ und den Ruck $j_{1}$ definiert. Die Anfangsbedingung $\underline{x}_{1}$ am Anfang des 2 . Segmentes bzw. am Ende des ersten Segmentes kann jetzt ebenfalls mit Hilfe von $\underline{\mathbf{x}}_{0}$ und $j_{1}$ dargestellt werden:

$$
\begin{aligned}
& \underline{\mathbf{x}}_{1}=\underbrace{\left[\begin{array}{cccc}
1 & T & T^{2} & T^{3} \\
0 & 1 & 2 T & 3 T^{2} \\
0 & 0 & 2 & 6 T
\end{array}\right]}_{\underline{\mathbf{C}}=\underline{\mathbf{F}}_{\mathrm{b}}^{\mathrm{T}}(T)(1: 3,:)} \cdot \underline{\mathbf{p}}_{1} \\
& \underline{\mathbf{x}}_{1}=\underline{\mathbf{C}} \cdot \underline{\mathbf{p}}_{1}
\end{aligned}
$$

Einsetzen von $\underline{\mathbf{p}}_{1}$ liefert:

$$
\underline{\mathbf{x}}_{1}=\underline{\mathbf{C}} \cdot \underline{\mathbf{A}} \cdot \underline{\mathbf{x}}_{0}+\underline{\mathbf{C}} \cdot \underline{\mathbf{b}} \cdot j_{1}
$$


Die Matrix $\underline{\mathbf{C}}$ enthält hierbei die ersten 3 Zeilen von $\underline{\mathbf{F}}_{\mathrm{b}}^{\mathrm{T}}(T)$. Für die weiteren Segmente ergeben sich die Polynomkoeffizienten wieder aus der Anfangsbedingung und dem Ruck. Durch Einsetzen von $\underline{\mathbf{x}}_{1}$ erhält man:

$$
\begin{aligned}
& \underline{\mathbf{p}}_{2}=\underline{\mathbf{A C A}}_{0}+\underline{\mathbf{x}}_{0}+\underline{\mathbf{A C b}} j_{1}+\underline{\mathbf{b}} j_{2} \\
& \underline{\mathbf{p}}_{2}=[\underline{\mathbf{A} C \underline{A}}] \underline{\mathbf{x}}_{0}+\left[\begin{array}{lllll}
\underline{\mathbf{A}} \underline{\mathbf{b}} & \underline{\mathbf{b}} & 0 & \ldots & 0
\end{array}\right] \quad\left[\begin{array}{c}
j_{1} \\
\vdots \\
j_{n}
\end{array}\right]
\end{aligned}
$$

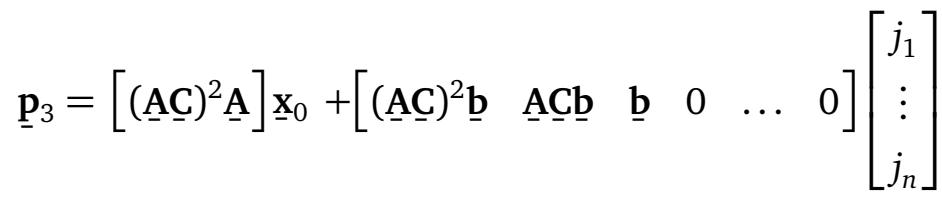

$$
\begin{aligned}
& \underline{\mathbf{p}}_{i}=\underbrace{\left[(\underline{\mathbf{A}} \underline{\mathbf{C}})^{i-1} \underline{\mathbf{A}}\right]}_{\underline{\mathbf{D}}_{i}}] \underline{\mathbf{x}}_{0}+\underbrace{\left[\begin{array}{llll}
(\underline{\mathbf{A}} \underline{\mathbf{C}})^{i-1} \underline{\mathbf{b}} & (\underline{\mathbf{A}} \underline{\mathbf{C}})^{i-2} \underline{\mathbf{b}} & \ldots & 0
\end{array}\right]}_{\underline{\mathbf{E}}_{i}} \underbrace{\left[\begin{array}{c}
j_{1} \\
\vdots \\
j_{n}
\end{array}\right]}_{\underline{\mathbf{j}}}
\end{aligned}
$$

Alle Polynomkoeffizienten setzen sich somit aus einem Term $\underline{\mathbf{D}}_{i} \underline{\mathbf{x}}_{0}$ basierend auf dem Anfangswert und einem variablem Term $\underline{\mathbf{E}}_{i} \mathbf{j}$ zusammen:

$$
\begin{aligned}
\underline{\mathbf{p}}_{i} & =\underline{\mathbf{D}}_{i} \underline{\mathbf{x}}_{0}+\underline{\mathbf{E}}_{i} \mathbf{j} \\
\underline{\mathbf{f}}_{i}(\tau) & =\underline{\mathbf{F}}_{\mathrm{b}}^{\mathrm{T}}(\tau) \underline{\mathbf{p}}_{i}
\end{aligned}
$$

Hierbei ist anzumerken, dass $\underline{\mathbf{A}}, \underline{\mathbf{b}}, \underline{\mathbf{C}}, \underline{\mathbf{D}}_{i}$ und $\underline{\mathbf{E}}_{i}$ nur von $T$ abhängen. Somit sind sie für eine feste Segmentlänge konstant.

\section{B.1.1 Integraler Güteterm}

Soll für den Spline ein quadratisches Gütemaß

$$
J_{A}=\int_{0}^{n T}\left[\underline{\mathbf{f}}^{\mathrm{T}}(t) \underline{\mathbf{W}}_{\mathrm{A}} \mathbf{f}(t)\right] \mathrm{d} t
$$

mit der diagonalen Gewichtungsmatrix $\underline{\mathbf{W}}_{\mathrm{A}}$ berechnet werden, so ist dies ebenfalls effizient möglich. Zuerst wird dazu der Beitrag $J_{i}$ eines einzelnen Segmentes bestimmt:

$$
\begin{aligned}
J_{i} & =\int_{0}^{T}\left[\underline{\mathbf{f}}_{i}^{\mathrm{T}}(\tau) \underline{\mathbf{W}}_{\mathrm{A}} \underline{\mathbf{f}}_{i}(\tau)\right] \mathrm{d} \tau \\
& =\int_{0}^{T}\left[\underline{\mathbf{p}}_{i}^{\mathrm{T}} \underline{\mathbf{F}}_{\mathrm{b}}(\tau) \underline{\mathbf{W}}_{\mathrm{A}} \underline{\mathbf{F}}_{\mathrm{b}}^{\mathrm{T}}(\tau) \underline{\mathbf{p}}_{i}\right] \mathrm{d} \tau \\
& =\underline{\mathbf{p}}_{i}^{\mathrm{T}} \underbrace{\int_{0}^{T}\left[\underline{\mathbf{F}}_{\mathrm{b}}(\tau) \underline{\mathbf{W}}_{\mathrm{A}} \underline{\mathbf{F}}_{\mathrm{b}}^{\mathrm{T}}(\tau)\right] \mathrm{d} \tau}_{\underline{\tilde{\mathbf{w}}}} \cdot \underline{\mathbf{p}}_{i} \\
& =\underline{\mathbf{p}}_{i}^{\mathrm{T}} \underline{\tilde{\mathbf{W}}} \underline{\mathbf{p}}_{i}
\end{aligned}
$$


Jetzt kann für $\underline{\mathbf{p}}_{i}=\underline{\mathbf{D}}_{i} \underline{\mathbf{x}}_{0}+\underline{\mathbf{E}}_{i} \underline{\mathbf{j}}$ eingesetzt werden:

$$
\begin{aligned}
& J_{i}=\underline{\mathbf{p}}_{i}^{\mathrm{T}} \underline{\tilde{\mathbf{W}}} \underline{\mathbf{p}}_{i} \\
& =\left(\underline{\mathbf{j}}^{\mathrm{T}} \underline{\mathbf{E}}_{i}^{\mathrm{T}}+\underline{\mathbf{x}}_{0}^{\mathrm{T}} \underline{\mathbf{D}}_{i}^{\mathrm{T}}\right) \underline{\tilde{\mathbf{w}}}\left(\underline{\mathbf{E}}_{i} \mathbf{j}+\underline{\mathbf{D}}_{i} \underline{\mathbf{x}}_{0}\right) \\
& =\underline{\mathbf{j}}^{\mathrm{T}} \underbrace{\underbrace{\mathrm{T}}_{i} \underline{\tilde{\mathbf{W}}} \underline{\mathbf{E}}_{i}}_{\underline{\mathbf{F}}_{i}} \underline{\mathbf{j}}+\underline{\mathbf{x}}_{0}^{\mathrm{T}} \underbrace{2 \underline{\underline{D}}_{i}^{\mathrm{T}} \tilde{\tilde{\mathbf{W}}} \underline{\mathbf{E}}_{i}}_{\underline{\mathbf{G}}_{i}} \underline{\mathbf{j}}+\underline{\mathbf{x}}_{0}^{\mathrm{T}} \underbrace{\mathbf{D}_{i}^{\mathrm{T}} \underline{\tilde{\mathbf{W}}} \underline{\mathbf{D}}_{i}}_{\underline{\mathbf{H}}_{i}} \underline{\mathbf{x}}_{0} \\
& =\underline{\mathbf{j}}^{\mathrm{T}} \underline{\mathbf{F}}_{i} \underline{\mathbf{j}}+\underline{\mathbf{x}}_{0}^{\mathrm{T}} \underline{\mathbf{G}}_{i} \underline{\mathbf{j}}+\underline{\mathbf{x}}_{0}^{\mathrm{T}} \underline{\mathbf{H}}_{i} \underline{\mathbf{x}}_{0}
\end{aligned}
$$

Für das integrale Gütemaß $J_{\mathrm{A}}$ des ganzen Splines ergibt sich dann:

$$
\begin{aligned}
& J_{\mathrm{A}}=\sum_{i=1}^{n} J_{i} \\
&=\underline{\mathbf{j}}^{\mathrm{T}} \underbrace{\underline{\mathbf{j}}}_{\sum_{i=1}^{n} \underline{\mathbf{F}}_{i}}+\underline{\mathbf{x}}_{0}^{\mathrm{T}} \underbrace{\sum_{i=1}^{n} \underline{\mathbf{G}}_{i}}_{\underline{\mathbf{F}}_{\mathrm{A}}} \underline{\mathbf{j}}_{\mathrm{A}}+\underline{\mathbf{x}}_{0}^{\mathrm{T}} \underbrace{\sum_{i=1}^{n} \underline{\mathbf{H}}_{i}}_{\underline{\mathbf{H}}_{\mathrm{A}}} \underline{\mathbf{x}}_{0} \\
&=\underline{\mathbf{j}}^{\mathrm{T}} \underline{\mathbf{F}}_{\mathrm{A}} \mathbf{j}+\underline{\mathbf{x}}_{0}^{\mathrm{T}} \underline{\mathbf{G}}_{\mathrm{A}} \underline{\mathbf{j}}+\underline{\mathbf{x}}_{0}^{\mathrm{T}} \underline{\mathbf{H}}_{\mathrm{A}} \underline{\mathbf{x}}_{0} \\
& \frac{\partial J_{\mathrm{A}}}{\partial \underline{\mathbf{j}}}=2 \underline{\mathbf{j}}^{\mathrm{T}} \underline{\mathbf{F}}_{\mathrm{A}}+\underline{\mathbf{x}}_{0}^{\mathrm{T}} \underline{\mathbf{G}}_{\mathrm{A}}
\end{aligned}
$$

Auch hier sind die Matrizen $\underline{\mathbf{F}}_{\mathrm{A}}, \underline{\mathbf{G}}_{\mathrm{A}}$ sowie $\underline{\mathbf{H}}_{\mathrm{A}}$ nur von der Segmentlänge $T$ sowie der Gewichtungsmatrix $\underline{\mathbf{W}}_{\mathrm{A}}$ abhängig. Daher können sie offline berechnet und im Speicher abgelegt werden.

\section{B.1.2 Endpunktbewertung}

In vielen Fällen kommt zum Gütemaß noch eine Bewertung $J_{\mathrm{B}}$ der Abweichung des Endpunktes $\underline{\mathbf{x}}_{n}$ des Splines von einem Wunschzustand $\underline{\mathbf{x}}_{\mathrm{e}}$ hinzu. Dies lässt sich einfach integrieren. Hierbei ist $\underline{\mathbf{W}}_{\mathrm{B}}$ eine diagonale Gewichtungsmatrix:

$$
J_{\mathrm{B}}=\left(\underline{\mathbf{x}}_{n}-\underline{\mathbf{x}}_{\mathrm{e}}\right)^{\mathrm{T}} \underline{\mathbf{W}}_{\mathrm{B}}\left(\underline{\mathbf{x}}_{n}-\underline{\mathbf{x}}_{\mathrm{e}}\right) \quad \underline{\mathbf{W}}_{\mathrm{B}} \text { diagonal }
$$

Einsetzen von $\underline{\mathbf{x}}_{n}=\underline{\mathbf{C}}_{\mathrm{n}}=\underline{\mathrm{CD}}_{n} \underline{\mathbf{x}}_{0}+\underline{\mathrm{CE}}_{n} \underline{\mathrm{j}}$ :

$$
\begin{aligned}
J_{\mathrm{B}} & =\left(\underline{\mathbf{C D}}_{n} \underline{\mathbf{x}}_{0}+\underline{\mathbf{C E}}_{n} \mathbf{j}-\underline{\mathbf{x}}_{\mathrm{e}}\right)^{\mathrm{T}} \underline{\mathbf{W}}_{\mathrm{B}}\left(\underline{\mathbf{C D}}_{n} \underline{\mathbf{x}}_{0}+\underline{\mathbf{C E}}_{n} \underline{\mathbf{j}}-\underline{\mathbf{x}}_{\mathrm{e}}\right) \\
& =\left(\underline{\mathbf{x}}_{0}^{\mathrm{T}} \underline{\mathbf{D}}_{n}^{\mathrm{T}} \underline{\mathbf{C}}^{\mathrm{T}}+\underline{\mathbf{j}}^{\mathrm{T}} \underline{\mathbf{E}}_{n}^{\mathrm{T}} \underline{\mathbf{C}}^{\mathrm{T}}-\underline{\mathbf{x}}_{\mathrm{e}}^{\mathrm{T}}\right) \underline{\mathbf{W}}_{\mathrm{B}}\left(\underline{\mathbf{C D}}_{n} \underline{\mathbf{x}}_{0}+\underline{\mathbf{C E}}_{n} \underline{\mathbf{j}}-\underline{\mathbf{x}}_{\mathrm{e}}\right)
\end{aligned}
$$

Ausmultiplizieren liefert:

$$
\begin{aligned}
& J_{\mathrm{B}}=+\underline{\mathbf{x}}_{0}^{\mathrm{T}} \underline{\mathbf{D}}_{n}^{\mathrm{T}} \underline{\mathbf{C}}^{\mathrm{T}} \underline{\mathbf{W}}_{\mathrm{B}} \underline{\mathbf{C}}_{\mathbf{n}} \underline{\mathbf{x}}_{0}+\underline{\mathbf{x}}_{0}^{\mathrm{T}} \underline{\mathbf{D}}_{n}^{\mathrm{T}} \underline{\mathbf{C}}^{\mathrm{T}} \underline{\mathbf{W}}_{\mathrm{B}} \underline{\mathbf{C}} \underline{E}_{n} \mathbf{j}-\underline{\mathbf{x}}_{0}^{\mathrm{T}} \underline{\mathbf{D}}_{n}^{\mathrm{T}} \underline{\mathbf{C}}^{\mathrm{T}} \underline{\mathbf{W}}_{\mathrm{B}} \underline{\mathbf{x}}_{\mathrm{e}} \\
& +\underline{\mathbf{j}}^{\mathrm{T}} \underline{\mathbf{E}}_{n}^{\mathrm{T}} \underline{\mathbf{C}}^{\mathrm{T}} \underline{\mathbf{W}}_{\mathrm{B}} \underline{\mathbf{C}}_{n} \underline{\mathbf{x}}_{0}+\underline{\mathbf{j}}^{\mathrm{T}} \underline{\mathbf{E}}_{n}^{\mathrm{T}} \underline{\mathbf{C}}^{\mathrm{T}} \underline{\mathbf{W}}_{\mathrm{B}} \underline{\mathbf{C E}} \underline{\mathbf{E}}_{n} \underline{\mathbf{j}}-\underline{\mathbf{j}}^{\mathrm{T}} \underline{\mathbf{E}}_{n}^{\mathrm{T}} \underline{\mathbf{C}}^{\mathrm{T}} \underline{\mathbf{W}}_{\mathrm{B}} \underline{\mathbf{x}}_{\mathrm{e}} \\
& -\underline{x}_{e}^{\mathrm{T}} \underline{\mathbf{W}}_{\mathrm{B}} \underline{\mathbf{C}}_{n} \underline{\mathbf{x}}_{0}-\quad \underline{\mathbf{x}}_{\mathrm{e}}^{\mathrm{T}} \underline{\mathbf{W}}_{\mathrm{B}} \underline{\mathbf{C E}}_{n} \mathbf{j}+\quad \underline{\mathrm{x}}_{\mathrm{e}}^{\mathrm{T}} \underline{\mathbf{W}}_{\mathrm{B}} \underline{\mathbf{x}}_{\mathrm{e}}
\end{aligned}
$$


Da $J_{\mathrm{B}}$ ein Skalar ist, lassen sich einige Terme zusammenfassen:

$$
\begin{aligned}
& J_{\mathrm{B}}=\underline{\mathbf{x}}_{0}^{\mathrm{T}} \underbrace{(\underbrace{\mathrm{T}}_{n} \mathbf{C}^{\mathrm{T}} \underline{\mathbf{W}}_{\mathrm{B}} \mathbf{C} \underline{\mathbf{D}}_{\mathrm{n}})}_{\underline{\mathbf{W}}_{\mathrm{B} 5}} \underline{\mathbf{x}}_{0}+\underline{\mathbf{x}}_{0}^{\mathrm{T}} \underbrace{\left(2 \underline{\mathbf{D}}_{n}^{\mathrm{T}} \underline{\mathbf{C}}^{\mathrm{T}} \underline{\mathbf{W}}_{\mathrm{B}} \mathbf{C E} \underline{E}_{n}\right)}_{\underline{\mathbf{W}}_{\mathrm{B} 2}} \underline{\mathbf{j}}^{\mathbf{j}}+\underline{\mathbf{x}}_{\mathrm{e}}^{\mathrm{T}} \underbrace{\left(-2 \underline{\mathbf{W}}_{\mathrm{B}} \mathbf{C E}\right.}_{\underline{\mathbf{W}}_{\mathrm{B} 3}}) \underline{\mathbf{j}}+ \\
& \underline{\mathbf{x}}_{\mathrm{e}}^{\mathrm{T}} \underbrace{\left(-2 \underline{\mathbf{W}}_{\mathrm{B}} \underline{C D}_{n}\right)}_{\underline{\mathbf{W}}_{\mathrm{B} 4}} \underline{\mathbf{x}}_{0}+\underline{\mathbf{j}}^{\mathrm{T}} \underbrace{\left(\underline{\mathbf{E}}_{n}^{\mathrm{T}} \underline{\mathbf{C}}^{\mathrm{T}} \underline{\mathbf{W}}_{\mathrm{B}} \underline{\mathbf{C E}} \underline{\mathbf{E}}_{n}\right)}_{\underline{\mathbf{W}}_{\mathrm{B} 1}} \underline{\mathbf{j}}^{\mathbf{W}}+\underline{\mathbf{x}}_{\mathrm{e}}^{\mathrm{T}} \underbrace{\left(\mathbf{W}_{\mathrm{B}}\right)}_{\underline{\mathbf{W}}_{\mathrm{B} 6}} \underline{\mathbf{x}}_{\mathrm{e}}
\end{aligned}
$$

Die Ableitung nach $\mathbf{j}$ lautet damit:

$$
\frac{\partial J_{\mathrm{B}}}{\partial \underline{\mathbf{j}}}=\underline{\mathbf{x}}_{0}^{\mathrm{T}} \underline{\mathbf{W}}_{\mathrm{B} 2}+\underline{\mathbf{x}}_{\mathrm{e}}^{\mathrm{T}} \underline{\mathbf{W}}_{\mathrm{B} 3}+2 \underline{\mathbf{j}}^{\mathrm{T}} \underline{\mathbf{W}}_{\mathrm{B} 1}
$$

\section{B.1.3 Abtastung}

Verwendete Symbole:

Symbol Beschreibung

$m \quad$ Anzahl Abtastpunkte pro Segment

$n \quad$ Anzahl Segmente

$T \quad$ Dauer eines Segmentes

Für einige Bewertungen müssen aus dem Spline Abtastpunkte bestimmt werden. Auch hier ist die Abhängigkeit der Abtastpunkte vom Parametervektor wichtig.

Es werden $m=2$ Abtastpunkte für jedes Polynomsegment verwendet. Für den gesamten Spline werden daher $m \cdot n=30$ Abtastpunkte verwendet. Der Startpunkt $\underline{\mathbf{x}}_{0}$ des Splines ist nicht in der Menge der Abtastpunkte enthalten (Abbildung B.2). Dies ist sinnvoll, da er durch die Planung sowieso nicht verändert werden kann.



Abbildung B.2: Die einzelnen Segmente einer Spline-Trajektorie zusammen mit den Abtastpunkten 
Die Abtastung wird hier für die Position gezeigt. Die Funktion $f_{\mathrm{p}}^{\mathrm{T}}(\tau)$ besteht hierbei aus der ersten Zeile der Matrixfunktion $\underline{\mathbf{F}}_{B}^{\mathrm{T}}(\tau)$. Für Geschwindigkeit, Beschleunigung und Ruck müssen die entsprechenden anderen Zeilen verwendet werden:

$$
\begin{aligned}
& s_{\mathrm{p} 1,1}= f_{\mathrm{p}}^{\mathrm{T}}\left(\frac{T}{m} \cdot 1\right) \underline{\mathbf{p}}_{1} \\
& s_{\mathrm{p} 1,2}= f_{\mathrm{p}}^{\mathrm{T}}\left(\frac{T}{m} \cdot 2\right) \underline{\mathbf{p}}_{1} \\
& \vdots \\
& s_{\mathrm{p} 1, m}= f_{\mathrm{p}}^{\mathrm{T}}\left(\frac{T}{m} \cdot m\right) \underline{\mathbf{p}}_{1}
\end{aligned}
$$

Die Abtastpunkte $\underline{\mathbf{s}}_{\mathrm{p} i}$ für das Segment $i$ können daher in Vektorform dargestellt werden:

$$
\underline{\mathbf{s}}_{\mathrm{p} i}=\underbrace{\left[\begin{array}{c}
f_{\mathrm{p}}^{\mathrm{T}}\left(\frac{T}{m} \cdot 1\right) \\
f_{\mathrm{p}}^{\mathrm{T}}\left(\frac{T}{m} \cdot 2\right) \\
\vdots \\
f_{\mathrm{p}}^{\mathrm{T}}\left(\frac{T}{m} \cdot m\right)
\end{array}\right]}_{\underline{\mathbf{K}}_{\mathrm{p}}} \underline{\mathbf{p}}_{i}
$$

Die Abtastpunkte $\underline{s}_{\mathrm{p}}$ für den gesamten Spline lassen sich dann wie folgt darstellen:

$$
\begin{aligned}
& \underline{\mathbf{s}}_{\mathrm{p}}=\left[\begin{array}{c}
s_{\mathrm{p} 1,1} \\
\vdots \\
s_{\mathrm{p} 1, m} \\
s_{\mathrm{p} 2,1} \\
\vdots \\
s_{\mathrm{p} n, m}
\end{array}\right]=\left[\begin{array}{cccc}
\underline{\mathbf{K}}_{\mathrm{p}} & \underline{\mathbf{0}} & \ldots & \underline{\mathbf{0}} \\
\underline{\mathbf{0}} & \underline{\mathbf{K}}_{\mathrm{p}} & \ddots & \vdots \\
\vdots & \ddots & \ddots & \underline{\mathbf{0}} \\
\underline{\mathbf{0}} & \ldots & \underline{\mathbf{0}} & \underline{\mathbf{K}}_{\mathrm{p}}
\end{array}\right]\left[\begin{array}{c}
\mathbf{p}_{1} \\
\underline{\mathbf{p}}_{2} \\
\vdots \\
\mathbf{p}_{n}
\end{array}\right]=\left[\begin{array}{cccc}
\underline{\mathbf{K}}_{\mathrm{p}} & \underline{\mathbf{0}} & \ldots & \underline{\mathbf{0}} \\
\underline{\mathbf{0}} & \underline{\mathbf{K}}_{\mathrm{p}} & \ddots & \vdots \\
\vdots & \ddots & \ddots & \underline{\mathbf{0}} \\
\underline{\mathbf{0}} & \ldots & \underline{\mathbf{0}} & \underline{\mathbf{K}}_{\mathrm{p}}
\end{array}\right]\left[\begin{array}{c}
\underline{\mathbf{D}}_{1} \underline{\mathbf{x}}_{0}+\underline{\mathbf{E}}_{1} \underline{\mathbf{j}} \\
\underline{\mathbf{D}}_{2} \underline{\mathbf{x}}_{0}+\underline{\mathbf{E}}_{2} \mathbf{j} \\
\vdots \\
\underline{\mathbf{D}}_{n} \underline{\mathbf{x}}_{0}+\underline{\mathbf{E}}_{n} \mathbf{j}
\end{array}\right] \\
&= {\left[\begin{array}{c}
\underline{\mathbf{K}}_{\mathrm{p}} \underline{\mathbf{D}}_{1} \underline{\mathbf{x}}_{0}+\underline{\mathbf{K}}_{\mathrm{p}} \underline{\mathbf{E}}_{1} \mathbf{j} \\
\underline{\mathbf{K}}_{\mathrm{p}} \underline{\mathbf{D}}_{2} \underline{\mathbf{x}}_{0}+\underline{\mathbf{K}}_{\mathrm{p}} \underline{\mathbf{E}}_{2} \mathbf{j} \\
\vdots \\
\underline{\mathbf{K}}_{\mathrm{p}} \underline{\mathbf{D}}_{n} \underline{\mathbf{x}}_{0}+\underline{\mathbf{K}}_{\mathrm{p}} \mathbf{E}_{n} \mathbf{j}
\end{array}\right]=\underbrace{\left[\begin{array}{c}
\underline{\mathbf{K}}_{\mathrm{p}} \underline{\mathbf{D}}_{1} \\
\underline{\mathbf{K}}_{\mathrm{p}} \underline{\mathbf{D}}_{2} \\
\vdots \\
\underline{\mathbf{K}}_{\mathrm{p}} \mathbf{D}_{n}
\end{array}\right]}_{\underline{\mathbf{L}}_{\mathrm{p}}} \underbrace{\left[\begin{array}{c}
\underline{\mathbf{K}}_{\mathrm{p}} \underline{\mathbf{E}}_{1} \\
\underline{\mathbf{K}}_{\mathrm{p}} \underline{\mathbf{E}}_{2} \\
\vdots \\
\underline{\mathbf{K}}_{\mathrm{p}} \mathbf{E}_{n}
\end{array}\right]}_{\underline{\mathbf{M}}_{\mathrm{p}}} }
\end{aligned}
$$

Die Matrizen $\underline{\mathbf{L}}_{\mathrm{p}}$ und $\underline{\mathbf{M}}_{\mathrm{p}}$ lassen sich hierbei vorab berechnen. Somit wird das Abtasten durch zwei Matrix-Multiplikationen realisiert:

$$
\begin{aligned}
\underline{\mathbf{s}}_{\mathrm{p}} & =\underline{\mathbf{L}}_{\mathrm{p}} \underline{\mathbf{x}}_{0}+\underline{\mathbf{M}}_{\mathrm{p}} \mathbf{j} \\
\frac{\partial \underline{\mathbf{s}}_{\mathrm{p}}}{\partial \underline{\mathbf{j}}} & =\underline{\mathbf{M}}_{\mathrm{p}}
\end{aligned}
$$

Die Abtastpunkte $\underline{\mathbf{s}}_{\mathrm{v}}$ für die Geschwindigkeit, $\underline{\mathbf{s}}_{\mathrm{a}}$ für die Beschleunigung sowie $\underline{\mathbf{s}}_{\mathrm{j}}$ für den Ruck ergeben sich analog dazu. 


\section{B.2 Alternative Parametrierungsmöglichkeiten}

Neben der Parametrierung der Trajektorie über die Rucke in den einzelnen Segmenten sind auch andere Parametrierungen denkbar. Die Parametrierung hat hierbei einen Einfluss auf die Konvergenz des nichtlinearen Optimierungsalgorithmus. Es ist wünschenswert, dass kleine Änderungen in den Parametern auch zu kleinen Änderungen in der Trajektorie führen.

\section{Parametrierung über Positionen unter Vorgabe von 3 Startbedingungen}

Als Alternative wurde die Verwendung der Positionen am Ende der einzelnen Segmente als Parameter untersucht. Zusätzlich dazu werden wie bisher die Größen $p, v$ und $a$ am Start der Trajektorie vorgegeben. Dabei hat sich gezeigt, dass der Spline die Punkte nicht wie vielleicht erwartet vergleichsweise glatt verbindet, sondern stark zu Oszillationen neigt (Abbildung B.3). Hierbei führen schon minimale Änderungen an einem Parameter zu sehr großen Änderungen in anderen Bereichen der Trajektorie. Dies ist nicht erwünscht und somit eine solche Parametrierung nicht geeignet.

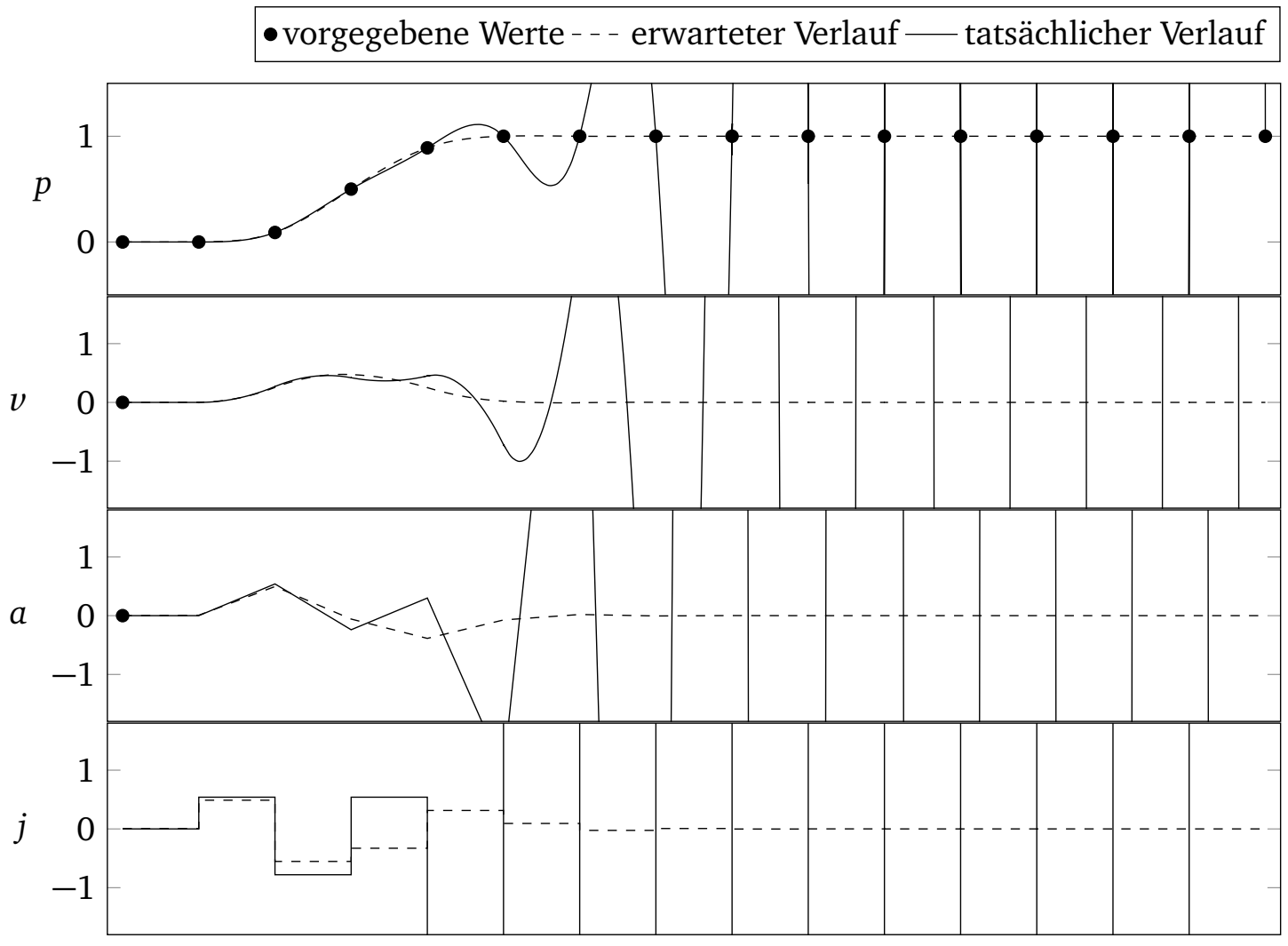

Abbildung B.3: Parametrierung des Splines über die Positionen an den Endpunkten der Segmente. Vorgabe von $p, v$ und $a$ als Startbedingung. Wie erkennbar ist, verhält sich der Spline nicht wie vielleicht vermutet, sondern neigt stark zu Oszillationen. 
Parametrierung über Positionen unter Vorgabe von 2 Startbedingungen und einer Endbedingung

Wird anstatt der Beschleunigung am Anfangspunkt die Geschwindigkeit am Endpunkt vorgegeben, so ist keine Neigung zu Oszillationen mehr zu erkennen. Selbst bei einem einzelnen von der exakten Trajektorie abweichenden Stützpunkt bleibt der Einfluss lokal begrenzt (Abbildung B.4). Diese Form der Parametrierung ist daher für die Quertrajektorie sehr interessant und sollte weiter untersucht werden. Für die Längstrajektorie ist sie weniger sinnvoll, da eine Endgeschwindigkeit vorgegeben werden muss.

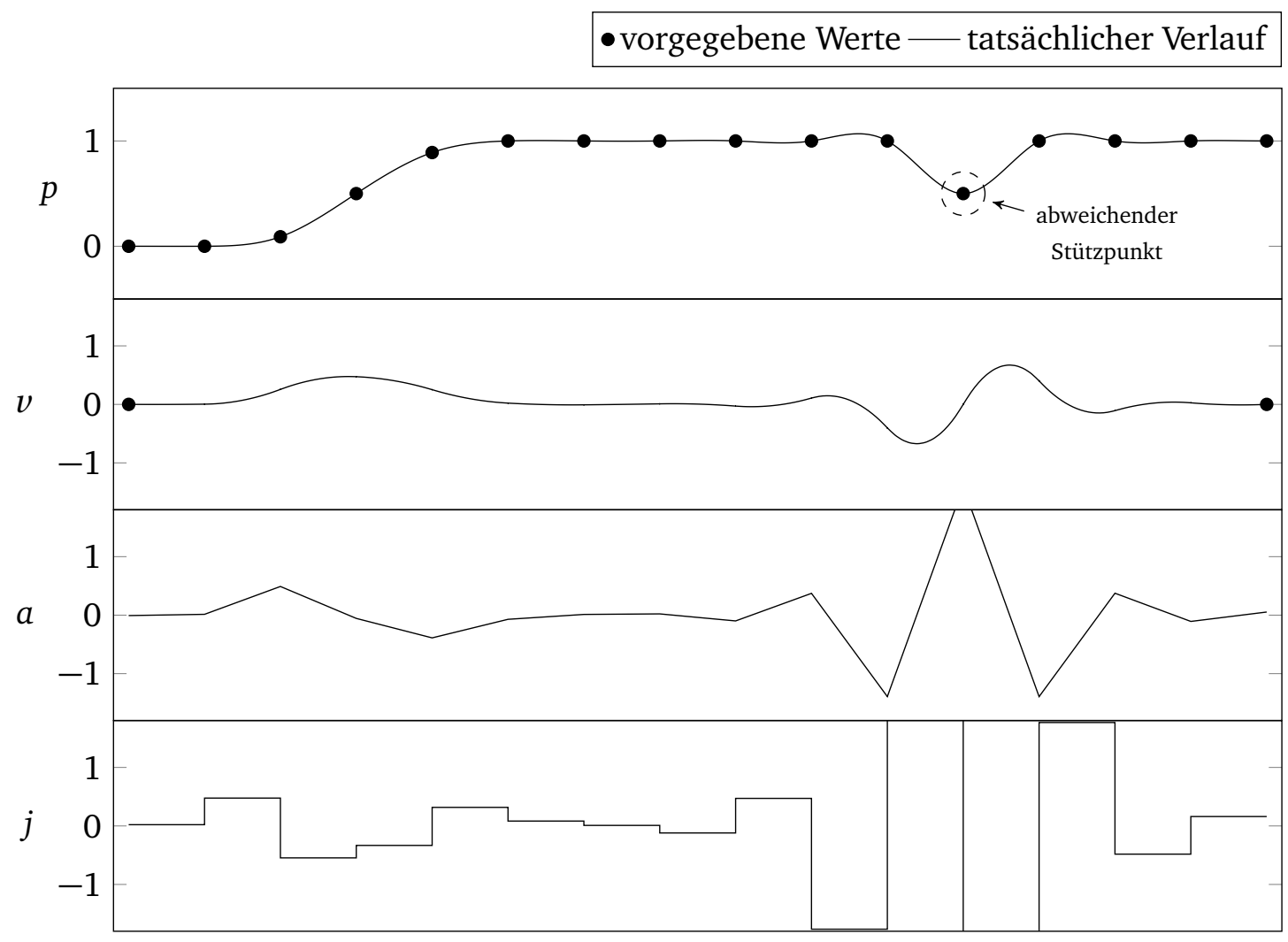

Abbildung B.4: Parametrierung des Splines über die Positionen an den Endpunkten der Segmente. Vorgabe von $p, v$ als Startbedingung und $v$ als Endbedingung. Wie erkennbar ist, ist keine Neigung zu Oszillationen vorhanden. Auch eine einzelne Stützstellenabweichung hat nur lokalen Einfluss. 


\section{B.3 Bestimmung der überschüssigen Energie am Endpunkt}

Idee:

Für die Optimierung ist es notwendig, dass die Straffunktion in der gesamten Ebene aus Abstand und Geschwindigkeit definiert und stetig differenzierbar ist. Gewählt wurde hierzu folgendes Maß:

„Überschüssige Energie, die noch abgebaut werden müsste, um dann durch Ausrollen den Sicherheitsabstand zu erreichen“

Der Ausrollvorgang wird hierbei näherungsweise als Verzögerung mit konstanter Beschleunigung $a=-0,41 \mathrm{~m} / \mathrm{s}^{2}$ betrachtet. Diese Verzögerung wurde mit Hilfe einer Abschätzung der Fahrwiderstände aus den Fahrzeugdaten für die Geschwindigkeit von $120 \mathrm{~km} / \mathrm{h}$ bestimmt. Sofern die Steigung der Fahrbahn bekannt ist, könnte diese entsprechend berücksichtigt werden.

Für die Berechnung des Sicherheitsabstands werden dieselben Parameter wie in Abschnitt 5.6.3 verwendet. Der Abstand des Ego-Fahrzeuges von diesem Sicherheitsabstand wird mit $d$ bezeichnet.

Betrachtet wird zunächst der Fall, dass sich das Ego-Fahrzeug am Endpunkt noch nicht im Sicherheitsabstand zum vorderen Fahrzeug befindet. Die Ego-Geschwindigkeit ist höher als die des vorausfahrenden Fahrzeugs, so dass die Grenze des Sicherheitsabstandes nach einiger Zeit erreicht würde.

Zur Bestimmung der überschüssigen Energie wird wie folgt vorgegangen:

- Der Endpunkt der Spline-Trajektorie liegt auf $t=t_{\mathrm{e}}$. Bis zu diesem Endpunkt kann das vordere Fahrzeug auch mit einem anderen Verfahren als $v=$ konst prädiziert werden.

- Es wird angenommen, dass sich das vordere Fahrzeug nach dem Zeitpunkt $t_{\mathrm{e}}$ mit konstanter Geschwindigkeit bewegt.

- Es soll die überschüssige Energie bestimmt werden, die zum Zeitpunkt $t_{\mathrm{e}}$ abgebaut werden müsste, um dann, nach einem Ausrollen bis zum Zeitpunkt $t_{2}$, dem vorderen Fahrzeug mit gleicher Geschwindigkeit und genau im Sicherheitsabstand zu folgen.

- Gesucht ist daher die Geschwindigkeit, die bei einem gegebenen Abstand und gegebenen Geschwindigkeiten der beiden Fahrzeuge dazu führt, dass das hintere Fahrzeug durch Ausrollen genau den Sicherheitsabstand erreicht.

\section{Bestimmung der Soll-Geschwindigkeit}

Wenn die Ego-Fahrzeugposition zum Zeitpunkt $t_{\mathrm{e}}$ in den Koordinatenursprung gelegt wird, vereinfacht sich das Problem. Das vordere Fahrzeug fährt in einem Abstand $d$ mit der Geschwindigkeit $m$ vor dem eigenen Fahrzeug. Das eigene Fahrzeug verzögert gleichmäßig mit 
der Beschleunigung $a$ und erreicht am Punkt $\mathrm{P}_{2}$ den Sicherheitsabstand des vorderen Fahrzeugs (Abbildung B.5).



Abbildung B.5: Bestimmung der Soll-Geschwindigkeit im $x(t)$-Diagramm

Ziel ist es jetzt, die passende Anfangsgeschwindigkeit $g^{\prime}(0)$ des eigenen Fahrzeugs zu bestimmen. Dazu wird zunächst der Punkt $\mathrm{P}_{2}$ bestimmt. Am Punkt $\mathrm{P}_{2}$ müssen die Geschwindigkeiten der beiden Fahrzeuge gleich sein:

$$
\begin{aligned}
g^{\prime}\left(t_{2}\right) & =f^{\prime}\left(t_{2}\right) \\
a\left(t_{2}-t_{2}\right)+b & =m \\
b & =m
\end{aligned}
$$

An Punkt $\mathrm{P}_{2}$ muss die Position des Ego-Fahrzeugs dem des Sicherheitsabstandes entsprechen:

$$
\begin{aligned}
g\left(t_{2}\right) & =f\left(t_{2}\right) \\
\frac{1}{2} a\left(t_{2}-t_{2}\right)^{2}+b t_{2}+c & =m t_{2}+d \\
m t_{2}+c & =m t_{2}+d \\
c & =d
\end{aligned}
$$

Da $m, d$ und $a$ bekannt sind, ergibt sich:

$$
g(t)=\frac{1}{2} a\left(t-t_{2}\right)^{2}+m t+d
$$


Jetzt wird der Punkt $\mathrm{P}_{1}$ in $g(t)$ eingesetzt, um $t_{2}$ zu bestimmen:

$$
\begin{aligned}
g(0) & =0 \\
\frac{1}{2} a\left(-t_{2}\right)^{2}+d & =0 \\
t_{2}^{2} & =\frac{-2 d}{a} \\
t_{2} & = \pm \sqrt{\frac{-2 d}{a}}
\end{aligned}
$$

da $t_{2}>0$ und $a<0$ :

$$
t_{2}=\sqrt{\frac{-2 d}{a}}
$$

Mit dem nun bekannten $t_{2}$ kann die Soll-Geschwindigkeit am Startpunkt bestimmt werden:

$$
\begin{aligned}
g^{\prime}(0) & =a\left(0-t_{2}\right)+m \\
& =-a \sqrt{\frac{-2 d}{a}}+m \\
& \left.=\sqrt{(-a)^{2} \frac{-2 d}{a}}+m\right) a<0 \\
& =\sqrt{-2 d a}+m=v_{\text {ideal }}(d)
\end{aligned}
$$

Jedem Abstand $d$ wird jetzt also eine Geschwindigkeit $v_{\text {ideal }}(d)$ zugeordnet (Abbildung B.6).

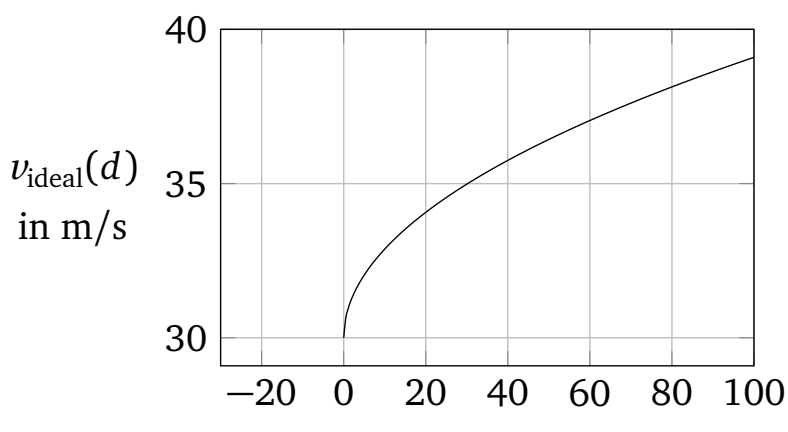

Distanz $d$ zum Sollabstand in $\mathrm{m}$

Abbildung B.6: Ideale Sollgeschwindigkeit $v_{\text {ideal }}(d)$ in Bezug zur Entfernung vom Sollabstand bei einer Geschwindigkeit des vorausfahrenden Fahrzeugs von $m=30 \mathrm{~m} / \mathrm{s}$

Problematisch ist hierbei der Bereich $d<0$. Für die Optimierung ist es notwendig, dass die Funktion in diesem Bereich sinnvoll definiert ist. Des Weiteren entspricht es auch einem natürlichen Verhalten eines menschlichen Fahrers, dass ein Teil des Bereiches $d<0$ befahren werden kann, sofern die Geschwindigkeit kleiner ist als die des vorderen Fahrzeuges. 


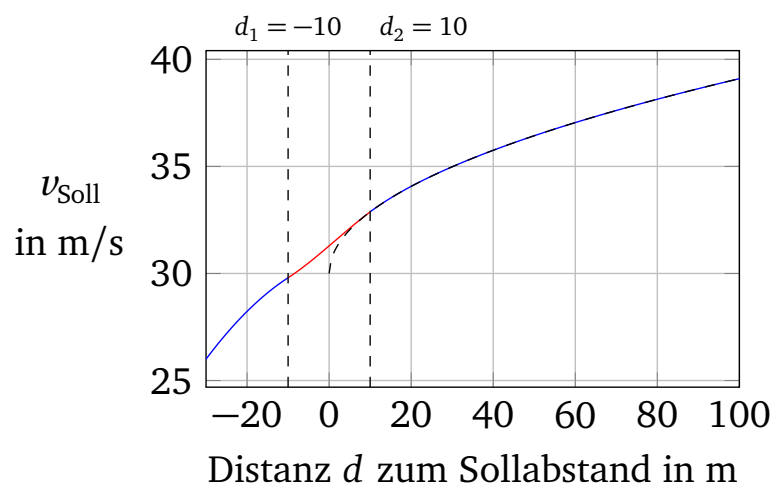

Abbildung B.7: Erweiterung der Funktion $v_{\text {Soll }}(d)$ auf den Bereich $d<0$

Daher findet eine stetig differenzierbare Erweiterung der Sollgeschwindigkeit statt. Aktuell geschieht dies in Form zweier kubischer Polynome mit den Koeffizienten $\mathbf{p}_{1}$ und $\mathbf{p}_{2}$ (Abbildung B.7):

$$
\begin{aligned}
x & =-2 a d \\
v_{\text {Soll }}(x) & =\left\{\begin{array}{llll}
m+\left[\begin{array}{llll}
x^{3} & x^{2} & x^{1} & 1
\end{array}\right] \underline{\mathbf{p}}_{\mathbf{1}} & d<d_{1} \\
m+\left[\begin{array}{llll}
x^{3} & x^{2} & x^{1} & 1
\end{array}\right] \underline{\mathbf{p}}_{2} & d \geq d_{1} \wedge d<d_{2} \\
m+\sqrt{x} & & d \geq d_{2}
\end{array}\right. \\
\frac{\mathrm{d} v_{\text {Soll }}}{\mathrm{d} d}(x) & =\left\{\begin{array}{llll}
{\left[\begin{array}{llll}
3 x^{2} & 2 x & 1 & 0
\end{array}\right] \underline{\mathbf{p}}_{1} \cdot(-2 a)} & d<d_{1} \\
{\left[\begin{array}{llll}
3 x^{2} & 2 x & 1 & 0
\end{array}\right] \underline{\mathbf{p}}_{2} \cdot(-2 a)} & d \geq d_{1} \wedge d<d_{2} \\
\frac{-1}{2 \sqrt{x}} \cdot(-2 a) & & d \geq d_{2}
\end{array}\right.
\end{aligned}
$$

\section{Bestimmung der Energiedifferenz}

Ausgehend von der Sollgeschwindigkeit kann nun eine Energiedifferenz bestimmt werden. Hierbei ist es wichtig, die Richtung der Geschwindigkeit zu berücksichtigen. Der einfache Ansatz $J_{\text {Diff }}=\frac{1}{2} m\left(v_{\mathrm{Ego}}^{2}-v_{\text {Soll }}^{2}\right)$ ist daher ungeeignet, was besonders für $v_{\mathrm{Ego}}=-v_{\text {Soll }}$ deutlich wird. Die Masse des Fahrzeugs kann ebenfalls entfallen, da sie im Rest der Trajektorienplanung nicht berücksichtigt wird und letztlich nur einen konstanten Faktor darstellt.

Daher wird folgende Variante verwendet:

$$
J_{\text {Diff }}=\frac{1}{2}\left(v_{\text {Ego }}^{2}-h\left(v_{\text {Soll }}\right)\right)
$$

mit

$$
h(x)=\left\{\begin{array}{ll}
-x^{2} & x<0 \\
x^{2} & x \geq 0
\end{array} \quad \frac{\partial h(x)}{\partial x}= \begin{cases}-2 x & x<0 \\
2 x & x \geq 0\end{cases}\right.
$$


Anschaulich entspricht dies der Tatsache, dass bei einer negativen Sollgeschwindigkeit erst die Ego-Geschwindigkeit abgebaut und dann die negative Sollgeschwindigkeit aufgebaut werden muss.

\section{Verlustenergie}

Interessant für die Endpunktbewertung ist nur die überschüssige Energie. Sofern noch zusätzliche Energie notwendig ist, um den Sollabstand zu erreichen, so ist dies nicht von Interesse. Daher müssten nur die positiven Werte betrachtet werden. Allerdings ist die Funktion $\max (x, 0)$ an der Stelle $x=0$ nicht stetig differenzierbar. Daher wird an dieser Stelle eine geglättete Funktion $\max _{\text {smooth }}(x)$ verwendet (Abbildung B.8):

$$
\begin{aligned}
& \Delta_{\mathrm{x}}=50 \mathrm{~m}^{2} / \mathrm{s}^{2} \\
& J_{\text {Lon,End,Loss }}=\max _{\text {smooth }}\left(J_{\text {Diff }}\right)= \begin{cases}0 & J_{\text {Diff }} \leq-\Delta_{\mathrm{x}} \\
\frac{1}{4 \Delta_{\mathrm{x}}}\left(J_{\text {Diff }}+\Delta_{\mathrm{x}}\right)^{2} & J_{\text {Diff }}>-\Delta_{\mathrm{x}} \wedge J_{\text {Diff }}<\Delta_{\mathrm{x}} \\
J_{\text {Diff }} & J_{\text {Diff }} \geq \Delta_{\mathrm{x}}\end{cases} \\
& \frac{\partial J_{\text {Lon,End,Loss }}}{\partial J_{\text {Diff }}}= \begin{cases}0 & J_{\text {Diff }} \leq-\Delta_{\mathrm{x}} \\
\frac{1}{2 \Delta_{\mathrm{x}}}\left(J_{\text {Diff }}+\Delta_{\mathrm{x}}\right) & J_{\text {Diff }}>-\Delta_{\mathrm{x}} \wedge J_{\text {Diff }}<\Delta_{\mathrm{x}} \\
1 & J_{\text {Diff }} \geq \Delta_{\mathrm{x}}\end{cases}
\end{aligned}
$$

Der Übergangsbereich $\pm \Delta_{\mathrm{x}}= \pm 50 \mathrm{~m}^{2} / \mathrm{s}^{2}$ entspricht bei einer Geschwindigkeit von $100 \mathrm{~km} / \mathrm{h}$ dabei etwa $\pm 6 \mathrm{~km} / \mathrm{h}$.

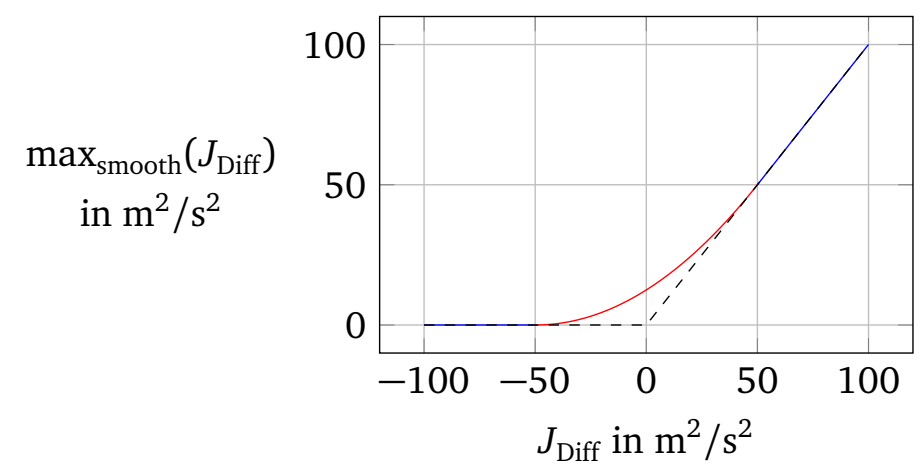

Abbildung B.8: Geglättete Funktion $\max _{\text {smooth }}(x)$

\section{Gesamtbetrachtung}

Betrachtet man nun die Gesamtfunktion $J_{\text {Lon,End,Loss }}\left(d, v_{\text {Ego }}, v_{\text {Front }}\right)$ (Abbildung B.9), so sieht man, dass sich eine Funktion ergibt, bei der der Punkt $v_{\text {Ego }}=v_{\text {Front }}, d=0$ in etwa am Rand des Anstieges liegt. Die Abweichungen, die durch die Glättung der Funktionen entstehen, heben sich für den Punkt $v_{\mathrm{Ego}}=v_{\text {Front }}, d=0$ fast vollständig auf. 


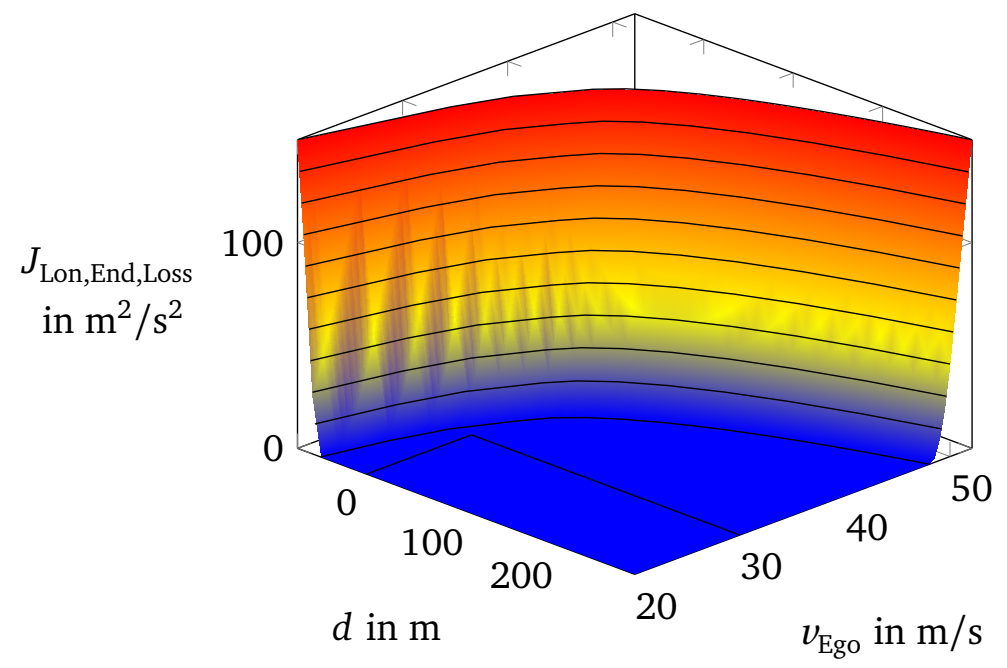

Abbildung B.9: Beispielhafter Verlauf von $J_{\text {Lon,End,Loss }}$ für eine Geschwindigkeit des vorderen Fahrzeugs von $v_{\text {Front }}=30 \mathrm{~m} / \mathrm{s}$. Am Schnittpunkt der beiden schwarzen Linien befindet sich der Punkt $v_{\text {Ego }}=v_{\text {Front }}, d=0$, für den $J_{\text {Lon,End,Loss }} \approx 0$ gilt.

\section{Alternativen}

Alternativ wäre es auch denkbar, die Geschwindigkeitsdifferenz quadratisch zu bestrafen:

$$
J_{\text {End,Loss,Alternativ }}= \begin{cases}\left(v_{\text {Ego }}-v_{\text {Soll }}\right)^{2} & v_{\text {Ego }} \geq v_{\text {Soll }} \\ 0 & v_{\text {Ego }}<v_{\text {Soll }}\end{cases}
$$

$\mathrm{Ob}$ und wenn ja welche Vor- und Nachteile dieses Vorgehen hat, müsste noch betrachtet werden. Der verfolgte Ansatz hat den Vorteil, dass die Bestrafung bei größeren Geschwindigkeiten höher ausfällt. 


\section{B.4 Fahrstreifenpotential}

Das Fahrstreifenpotential soll stetig differenzierbar, aber auch einfach zu berechnen sein. Es setzt sich aus zwei Komponenten zusammen, die unterschiedlich gewichtet werden können. Ziel der ersten Komponente ist es, ein längeres Fahren auf zwei Fahrstreifen gleichzeitig zu vermeiden. Die zweite Komponente sorgt für einen zügigen Wechsel auf den Zielfahrstreifen.

\section{Erste Teilkomponente}

Zur Generierung des Fahrstreifenpotentials wird jeder Fahrstreifen in vier Abschnitte gleicher Breite unterteilt. In jedem dieser Bereiche gilt eine quadratische Funktion (Abbildung B.10). $l_{1}$ und $l_{5}$ stehen hierbei für die Position der Fahrstreifenmarkierungen, $l_{3}$ für die Fahrstreifenmitte und $b_{\mathrm{FS}}$ für die Breite des Fahrstreifens:

$$
\phi_{\mathrm{FS}}\left(p_{\mathrm{y}}\right)=\left\{\begin{array}{ll}
2-a y_{1}^{2} & l_{1} \leq p_{\mathrm{y}}<l_{2} \\
a y_{2}^{2} & l_{2} \leq p_{\mathrm{y}}<l_{4} \\
2-a y_{3}^{2} & l_{4} \leq p_{\mathrm{y}}<l_{5}
\end{array} \quad a=\left(\frac{4}{b_{\mathrm{FS}}}\right)^{2}\right.
$$

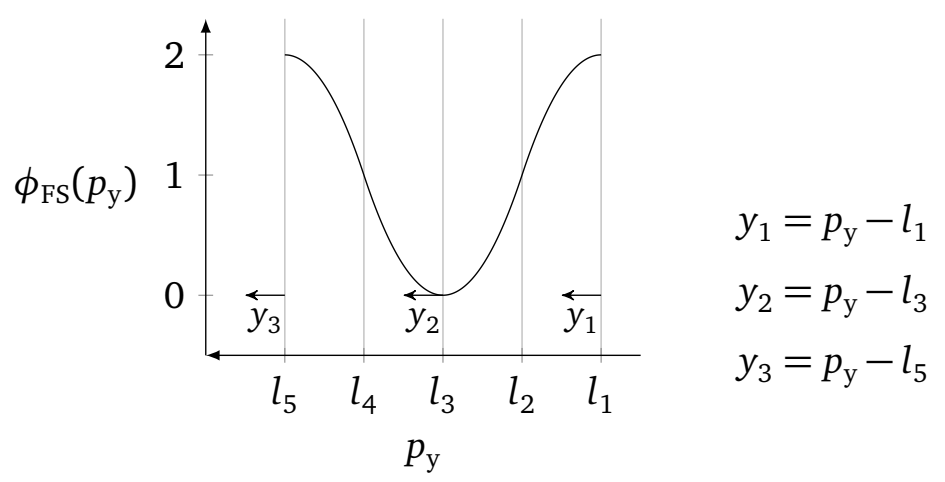

Abbildung B.10: Erste Teilkomponente der Fahrstreifenpotentialfunktion für einen Fahrstreifen. Man beachte die nach links positiv zählende Querposition.

Falls mehrere Fahrstreifen erkannt werden, entsteht ein Gesamtpotential durch Zusammensetzen aus den Einzelpotentialen. Hierbei wird die Breite der jeweiligen Fahrstreifen berücksichtigt (Abbildung B.11).

\section{Zweite Teilkomponente}

Die zweite Teilkomponente bestraft quadratisch die Abweichung der Querposition $p_{\mathrm{y}}$ von der Zielfahrstreifenmitte $p_{\text {y,ZFSM }}$ (Abbildung B.12):

$$
\phi_{\mathrm{FSM}}\left(p_{\mathrm{y}}\right)=\left(p_{\mathrm{y}}-p_{\mathrm{y}, \mathrm{ZFSM}}\right)^{2}
$$




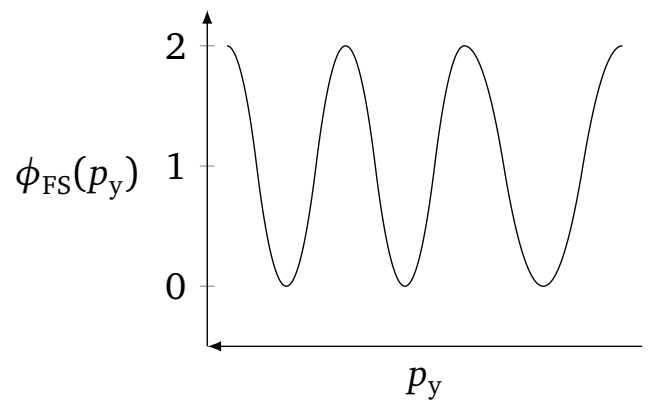

Abbildung B.11: Erste Teilkomponente des Fahrstreifenpotentials für mehrere Fahrstreifen. Der rechte Fahrstreifen ist in diesem Fall breiter als die beiden linken

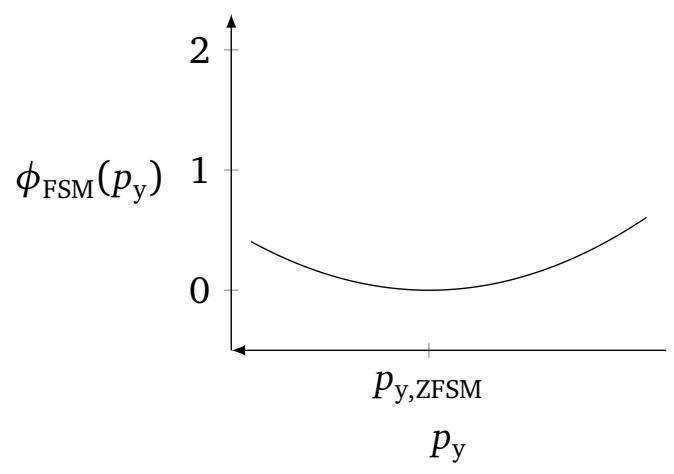

Abbildung B.12: Zweite Teilkomponente des Fahrstreifenpotentials

\section{Kombiniertes Fahrstreifenpotential}

Werden diese beiden Teilkomponenten nun gewichtet addiert, so erhält man das kombinierte Fahrstreifenpotential (Abbildung B.13, Abbildung B.14):

$$
\phi_{\mathrm{FSP}}\left(p_{\mathrm{y}}\right)=w_{\mathrm{FS}} \cdot \phi_{\mathrm{FS}}\left(p_{\mathrm{y}}\right)+w_{\mathrm{FSM}} \cdot \phi_{\mathrm{FSM}}\left(p_{\mathrm{y}}\right)
$$

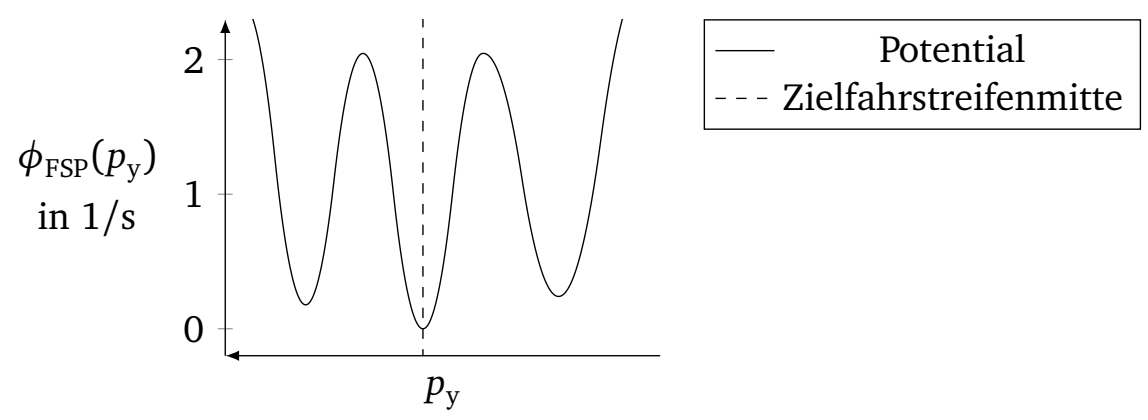

Abbildung B.13: Kombiniertes Fahrstreifenpotential 


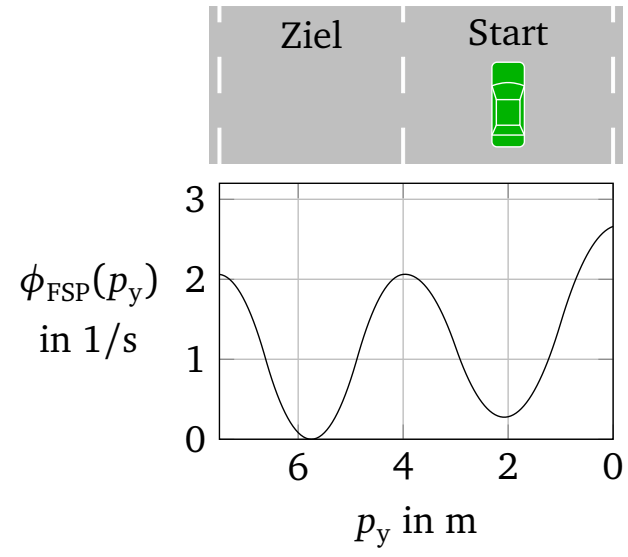

Abbildung B.14: Beispielhaftes Fahrstreifenpotential zusammen mit Start- und Zielfahrstreifen

\section{Integrationsmethode}

Verwendete Symbole:

Symbol Beschreibung

$y(t) \quad$ Lineare Approximation für die Querposition $p_{\mathrm{y}}(t)$ zwischen den Abtastpunkten

$y_{i} \quad$ Querposition zum Zeitpunkt $t_{i}$

$\phi_{\mathrm{FSP}} \quad$ Fahrstreifenpotentialfunktion

$\Phi_{\mathrm{FSP}} \quad$ Stammfunktion der Fahrstreifenpotentialfunktion

$J_{\text {FSP }} \quad$ Gütemaß der gesamten Trajektorie für das Fahrstreifenpotential

$J_{\mathrm{FSP}, \mathrm{i}} \quad$ Gütemaß der Trajektorie zwischen $t_{i}$ und $t_{i+1}$ für das Fahrstreifenpotential

$\Delta T_{\text {Samp }} \quad$ Zeitlicher Abstand zwischen zwei Abtastpunkten

Es hat sich gezeigt, dass eine reine Summe der Potentialwerte an den Abtastpunkten des Splines zu Problemen führt. Da aufgrund der knappen Rechenzeit nur wenig Abtastpunkte verwendet werden können, kommt es bei der Approximation

$$
J_{\mathrm{FSP}}=\int_{t_{0}}^{t_{\mathrm{e}}}\left[\phi_{\mathrm{FSP}}\left(p_{\mathrm{y}}(t)\right)\right] \mathrm{d} t \approx \sum_{i} \phi_{\mathrm{FSP}}\left(p_{\mathrm{y}, \mathrm{i}}\right) \Delta T_{\mathrm{Samp}}
$$

zu Ungenauigkeiten. Da lediglich das Potential an den Abtastpunkten betrachtet wird, geht ein Potentialberg zwischen den Abtastpunkten nicht in das Gütemaß ein. Diese Ungenauigkeiten führen dazu, dass Nebenminima entstehen, und das globale Minimum der Straffunktion nicht gefunden wird (Abbildung B.15). In der Praxis wird der Fahrstreifenwechsel dann unnötig lange hinausgezögert.

Um diese Unzulänglichkeiten zu vermeiden, wird das Integral auf eine bessere Art approximiert. Dazu wird für die Trajektorie zwischen den Abtastpunkten $y_{i}$ ein linearer Verlauf $y(t)$ angenommen:

$$
\begin{aligned}
y(t) & =\frac{y_{i+1}-y_{i}}{t_{i+1}-t_{i}} \cdot\left(t-t_{i}\right)+y_{i} \quad \text { jeweils im Bereich } t_{i} \leq t \leq t_{i+1} \\
\frac{\partial y(t)}{\partial t} & =\frac{y_{i+1}-y_{i}}{t_{i+1}-t_{i}}
\end{aligned}
$$



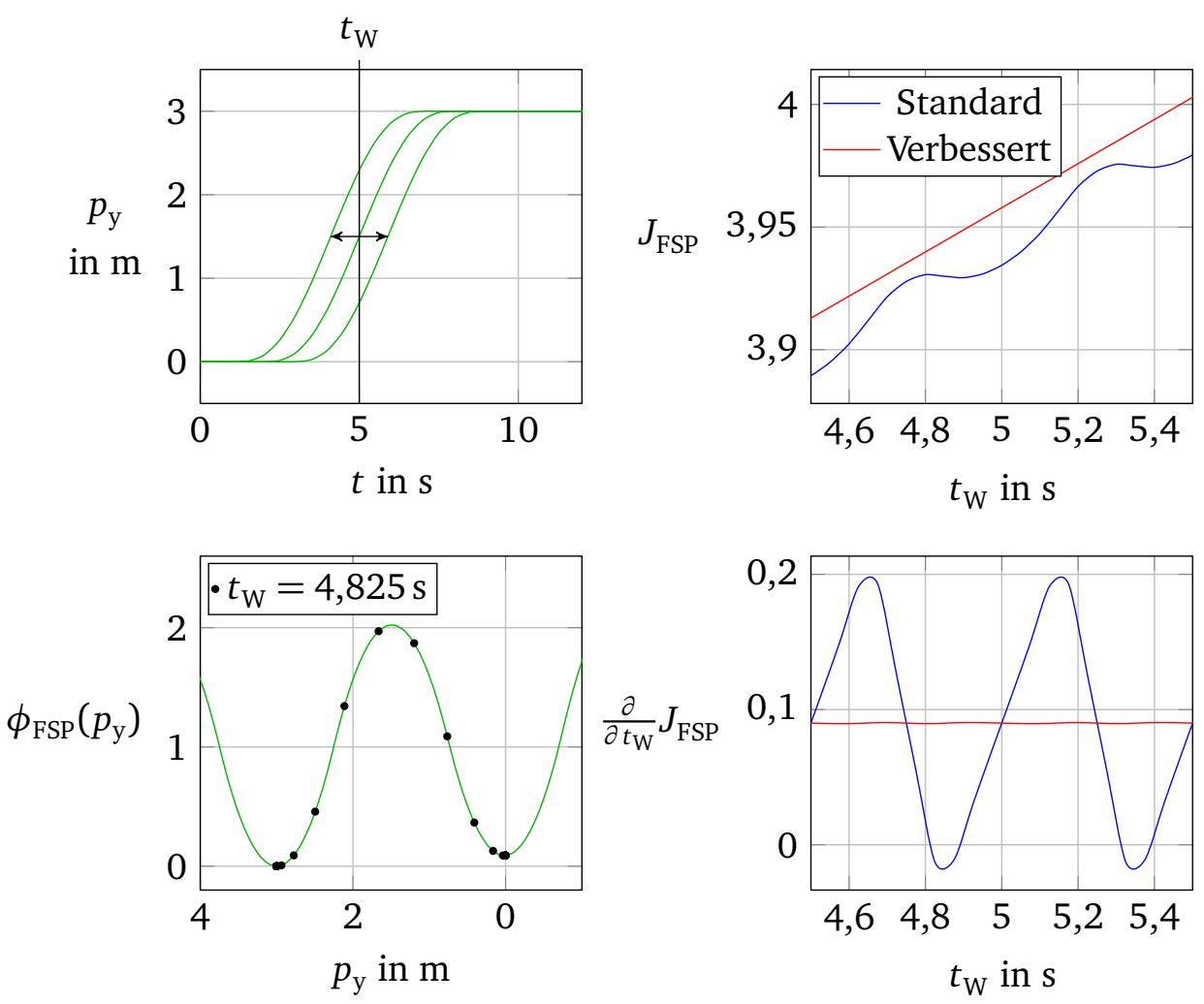

Abbildung B.15: Vergleich der Integrationsmethoden für das Fahrstreifenpotential. Variiert wird dabei der Wechselzeitpunkt $t_{\mathrm{W}}$

Für jedes dieser linearen Stücke lässt sich nun das Integral über die Fahrstreifenpotentialfunktion exakt bestimmen:

$$
\begin{array}{rlr}
J_{F S P} & =\int_{t_{0}}^{t_{\mathrm{e}}} \phi_{\mathrm{FSP}}\left(p_{y}(t)\right) \mathrm{d} t & n=\text { Anzahl Abtastpunkte } \\
& \approx \sum_{i=0}^{n-1} \underbrace{\left[\int_{t_{i}}^{t_{i+1}}\left[\phi_{\mathrm{FSP}}(y(t))\right] \mathrm{d} t\right]}_{J_{\mathrm{FSP}, \mathrm{i}}}=\sum_{i=0}^{n-1} J_{\mathrm{FSP}, \mathrm{i}} &
\end{array}
$$

Die Verwendung der Stammfunktion $\Phi_{\mathrm{FSP}}$ erleichtert hierbei die Arbeit mit der abschnittsweise definierten Potentialfunktion $\phi_{\mathrm{FSP}}$ :

$$
\begin{aligned}
J_{\mathrm{FSP}, \mathrm{i}} & =\int_{t_{i}}^{t_{i+1}}\left[\phi_{\mathrm{FSP}}(y(t))\right] \mathrm{d} t \\
& =\int_{y_{i}}^{y_{i+1}}\left[\phi_{\mathrm{FSP}}(y)\right] \mathrm{d} y \cdot \frac{t_{i+1}-t_{i}}{y_{i+1}-y_{i}} \\
& =\left[\Phi_{\mathrm{FSP}}(y)\right]_{y_{i}}^{y_{i+1}} \cdot \frac{t_{i+1}-t_{i}}{y_{i+1}-y_{i}}
\end{aligned}
$$


Damit ergibt sich:

$$
\begin{aligned}
\Delta T_{\mathrm{Samp}} & =t_{i+1}-t_{i} \\
J_{\mathrm{FSP}, \mathrm{i}} & =\frac{\Phi_{\mathrm{FSP}}\left(y_{i+1}\right)-\Phi_{\mathrm{FSP}}\left(y_{i}\right)}{y_{i+1}-y_{i}} \cdot \Delta T_{\mathrm{Samp}} \\
\frac{\partial J_{\mathrm{FSP}, \mathrm{i}}}{\partial y_{i}} & =\frac{-\phi_{\mathrm{FSP}}\left(y_{i}\right) \cdot\left(y_{i+1}-y_{i}\right)+\left[\Phi_{\mathrm{FSP}}\left(y_{i+1}\right)-\Phi_{\mathrm{FSP}}\left(y_{i}\right)\right]}{\left(y_{i+1}-y_{i}\right)^{2}} \cdot \Delta T_{\mathrm{Samp}} \\
\frac{\partial J_{\mathrm{FSP}, \mathrm{i}}}{\partial y_{i+1}} & =\frac{\phi_{\mathrm{FSP}}\left(y_{i+1}\right) \cdot\left(y_{i+1}-y_{i}\right)-\left[\Phi_{\mathrm{FSP}}\left(y_{i+1}\right)-\Phi_{\mathrm{FSP}}\left(y_{i}\right)\right]}{\left(y_{i+1}-y_{i}\right)^{2}} \cdot \Delta T_{\mathrm{Samp}}
\end{aligned}
$$

\section{Für kleine $\Delta y$}

Für $y_{i+1}=y_{i}$ wird jedoch durch 0 geteilt. Zudem ist für kleine Differenzen zwischen $y_{i+1}$ und $y_{i}$ die Form numerisch ungünstig. Um zu einer besseren Approximation zu gelangen, werden die Gleichungen zunächst auf die Mitte $\bar{y}_{i}$ zwischen $y_{i+1}$ und $y_{i}$ bezogen:

$$
\begin{aligned}
\bar{y}_{i} & =\frac{1}{2}\left(y_{i+1}+y_{i}\right) \\
\epsilon_{i} & =\frac{1}{2}\left(y_{i+1}-y_{i}\right) \\
J_{\mathrm{FSP}, \mathrm{i}} & =\frac{\Phi_{\mathrm{FSP}}\left(\bar{y}_{i}+\epsilon_{i}\right)-\Phi_{\mathrm{FSP}}\left(\bar{y}_{i}-\epsilon_{i}\right)}{2 \epsilon_{i}} \cdot \Delta T_{\mathrm{Samp}} \\
\frac{\partial J_{\mathrm{FSP}, \mathrm{i}}}{\partial y_{i}} & =\frac{-\phi_{\mathrm{FSP}}\left(\bar{y}_{i}-\epsilon_{i}\right) \cdot\left(2 \epsilon_{i}\right)+\left[\Phi_{\mathrm{FSP}}\left(\bar{y}_{i}+\epsilon_{i}\right)-\Phi_{\mathrm{FSP}}\left(\bar{y}_{i}-\epsilon_{i}\right)\right]}{4 \epsilon_{i}^{2}} \cdot \Delta T_{\mathrm{Samp}} \\
\frac{\partial J_{\mathrm{FSP}, \mathrm{i}}}{\partial y_{i+1}} & =\frac{\phi_{\mathrm{FSP}}\left(\bar{y}_{i}+\epsilon_{i}\right) \cdot\left(2 \epsilon_{i}\right)-\left[\Phi_{\mathrm{FSP}}\left(\bar{y}_{i}+\epsilon_{i}\right)-\Phi_{\mathrm{FSP}}\left(\bar{y}_{i}-\epsilon_{i}\right)\right]}{4 \epsilon_{i}^{2}} \cdot \Delta T_{\mathrm{Samp}}
\end{aligned}
$$

Durch Grenzwertbildung für $\epsilon_{i} \rightarrow 0$ erhält man nun unter Anwendung der Regel von de l'Hospital:

$$
\begin{aligned}
\lim _{\epsilon_{i} \rightarrow 0} J_{\mathrm{FSP}, \mathrm{i}} & =\frac{\phi_{\mathrm{FSP}}\left(\bar{y}_{i}+\epsilon_{i}\right)+\phi_{\mathrm{FSP}}\left(\bar{y}_{i}-\epsilon_{i}\right)}{2} \cdot \Delta T_{\mathrm{Samp}} \\
& =\phi_{\mathrm{FSP}}\left(\bar{y}_{i}\right) \cdot \Delta T_{\mathrm{Samp}}
\end{aligned}
$$

und für die Ableitung nach $y_{i}$ :

$$
\begin{aligned}
& \lim _{\epsilon_{i} \rightarrow 0} \frac{\partial J_{\mathrm{FSP}, \mathrm{i}}}{\partial y_{i}} \\
= & \lim _{\epsilon_{i} \rightarrow 0} \frac{\phi_{\mathrm{FSP}}^{\prime}\left(\bar{y}_{i}-\epsilon_{i}\right) \cdot 2 \epsilon_{i}-2 \phi_{\mathrm{FSP}}\left(\bar{y}_{i}-\epsilon_{i}\right)+\phi_{\mathrm{FSP}}\left(\bar{y}_{i}+\epsilon_{i}\right)+\phi_{\mathrm{FSP}}\left(\bar{y}_{i}-\epsilon_{i}\right)}{8 \epsilon_{i}} \cdot \Delta T_{\mathrm{Samp}} \\
= & \lim _{\epsilon_{i} \rightarrow 0} \frac{\phi_{\mathrm{FSP}}^{\prime}\left(\bar{y}_{i}-\epsilon_{i}\right) \cdot 2 \epsilon_{i}-\phi_{\mathrm{FSP}}\left(\bar{y}_{i}-\epsilon_{i}\right)+\phi_{\mathrm{FSP}}\left(\bar{y}_{i}+\epsilon_{i}\right)}{8 \epsilon_{i}} \cdot \Delta T_{\mathrm{Samp}} \\
= & \lim _{\epsilon_{i} \rightarrow 0} \frac{\phi_{\mathrm{FSP}}^{\prime \prime}\left(\bar{y}_{i}-\epsilon_{i}\right) \cdot(-1) \cdot 2 \epsilon_{i}+2 \phi_{\mathrm{FSP}}^{\prime}\left(\bar{y}_{i}-\epsilon_{i}\right)+\phi_{\mathrm{FSP}}^{\prime}\left(\bar{y}_{i}-\epsilon_{i}\right)+\phi_{\mathrm{FSP}}^{\prime}\left(\bar{y}_{i}+\epsilon_{i}\right)}{8} \cdot \Delta T_{\mathrm{Samp}} \\
= & \lim _{\epsilon_{i} \rightarrow 0} \frac{\phi_{\mathrm{FSP}}^{\prime \prime}\left(\bar{y}_{i}-\epsilon_{i}\right) \cdot(-1) \cdot 2 \epsilon_{i}+3 \phi_{\mathrm{FSP}}^{\prime}\left(\bar{y}_{i}-\epsilon_{i}\right)+\phi_{\mathrm{FSP}}^{\prime}\left(\bar{y}_{i}+\epsilon_{i}\right)}{8} \cdot \Delta T_{\mathrm{Samp}} \\
= & \frac{1}{2} \phi_{\mathrm{FSP}}^{\prime}\left(\bar{y}_{i}\right) \cdot \Delta T_{\mathrm{Samp}}
\end{aligned}
$$


Analog dazu ergibt sich für die Ableitung nach $y_{i+1}$ :

$$
\lim _{\epsilon_{i} \rightarrow 0} \frac{\partial J_{\mathrm{FSP}, \mathrm{i}}}{\partial y_{i+1}}=\frac{1}{2} \phi_{\mathrm{FSP}}^{\prime}\left(\bar{y}_{i}\right) \cdot \Delta T_{\mathrm{Samp}}
$$

Es hat sich gezeigt, dass es für $\left|y_{i+1}-y_{i}\right|<10^{-4} \mathrm{~m}$ sinnvoll ist, diese Grenzwerte zu verwenden. 


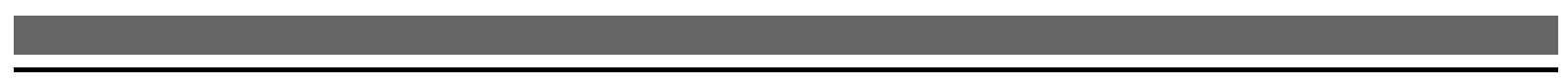




\section{Vergleich der Trajektorienplanungen in unterschiedlichen Szenarien}

Das folgende Kapitel zeigt die geplanten Trajektorien und Sollzustandsverläufe der beiden Planungen für eine Reihe unterschiedlicher Szenarien. „ACC“ kennzeichnet hierbei die Sollposition hinter dem vorausfahrenden Fahrzeug auf dem Zielfahrstreifen, auf die das ACC bei der Drei-Segment-Planung regelt. Ebenfalls eingezeichnet sind die Sicherheitsbereichsgrenzen der Drei-Segment-Planung. Diese haben für die Spline-Planung keine Bedeutung.

Durch die zyklische Neuplanung kann der ausgegebene Sollzustandsverlauf von der ursprünglich geplanten Trajektorie abweichen. Dies gilt insbesondere, wenn das Umfeld auf den Fahrstreifenwechsel reagiert. Diese Reaktion ist abhängig von der gewählten Trajektorie und simulativ nur schwer darzustellen. Hierfür sei daher auf die Ergebnisse der Testfahrten in Abschnitt 4.7 und Abschnitt 5.9 verwiesen. Zum Zwecke der Vergleichbarkeit werden hier Umfeldfahrzeuge mit konstanter Geschwindigkeit betrachtet. Dargestellt ist jeweils gestrichelt die im ersten Planungsschritt bestimmte Trajektorie sowie der sich durch die zyklische Neuplanung insgesamt ergebende Sollzustandsverlauf mit einer durchgezogenen Linie.

Dargestellt werden für jede Situation die Längsposition relativ zu einem bewegten Beobachter, die Querposition sowie die Längsbeschleunigung. Deutlich zu erkennen ist die unterschiedliche Länge der Trajektorie bei der Drei-Segment-Planung. Die Längstrajektorie der Drei-SegmentPlanung endet, wenn die Querbewegung weitestgehend abgeschlossen ist. Die Spline-Planung hat im Vergleich dazu eine feste Länge von $15 \mathrm{~s}$. 


\section{Szenario 1}

Ziellücke

$100 \mathrm{~km} / \mathrm{h}$
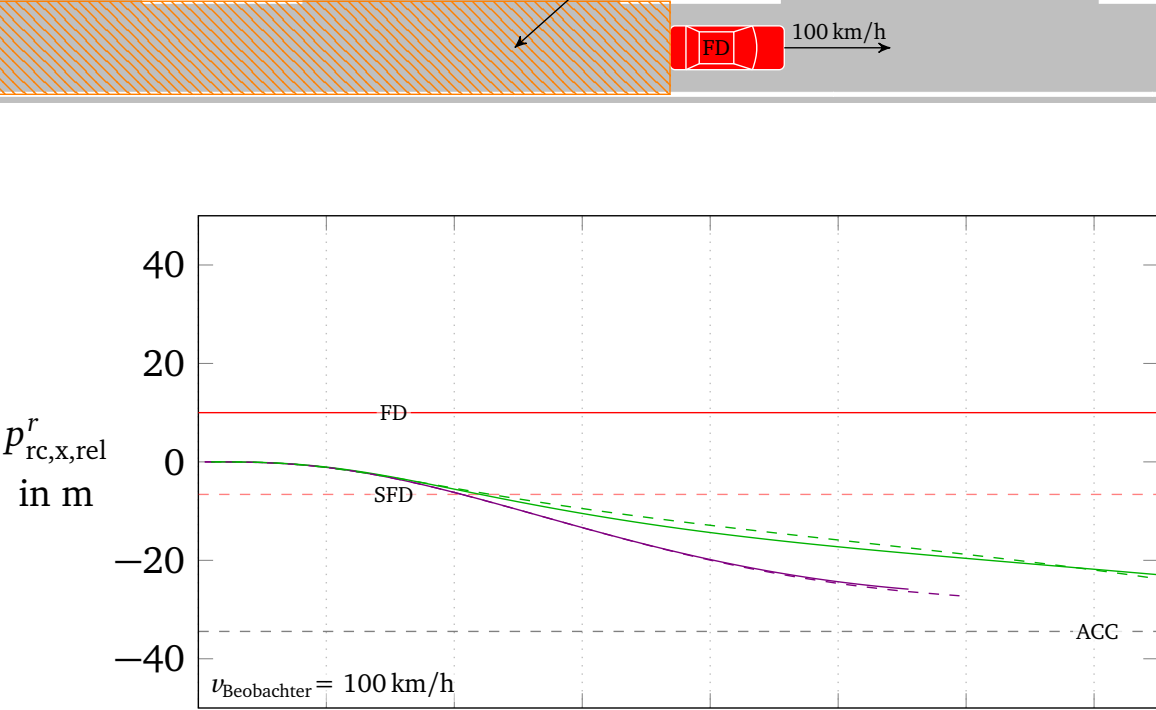

FD

Sich.-Ber. FD

ACC

3-Seg.-Sollz.

Spline-Sollz.

3-Seg.-Traj.

Spline-Traj.
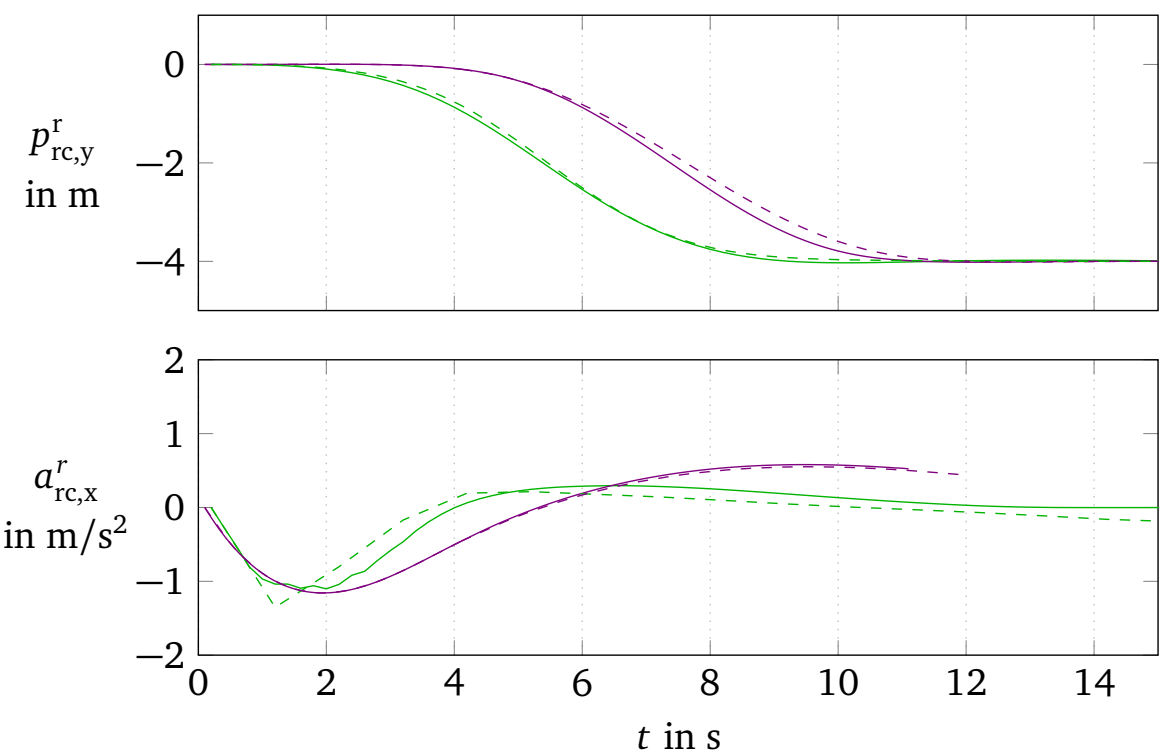

Abbildung C.1: Szenario 1: Ego fährt links neben dem Sicherheitsbereich von FD mit gleicher Geschwindigkeit. Zum Fahrstreifenwechsel soll es leicht verzögern und hinter FD einfädeln.

Im ersten Szenario befindet sich das Ego-Fahrzeug auf dem linken Fahrstreifen auf Höhe des Sicherheitsabstandes von FD (Abbildung C.1). Ziel ist der Wechsel auf den rechten Fahrstreifen hinter FD. Dazu muss zunächst verzögert und anschließend gewechselt werden. Es ist erkennbar, dass die Spline-Planung den Fahrstreifenwechsel früher beginnt. Dies liegt an der berücksichtigten Relativgeschwindigkeit zum vorausfahrenden Fahrzeug (Abschnitt 5.6.3). 


\section{Szenario 2}
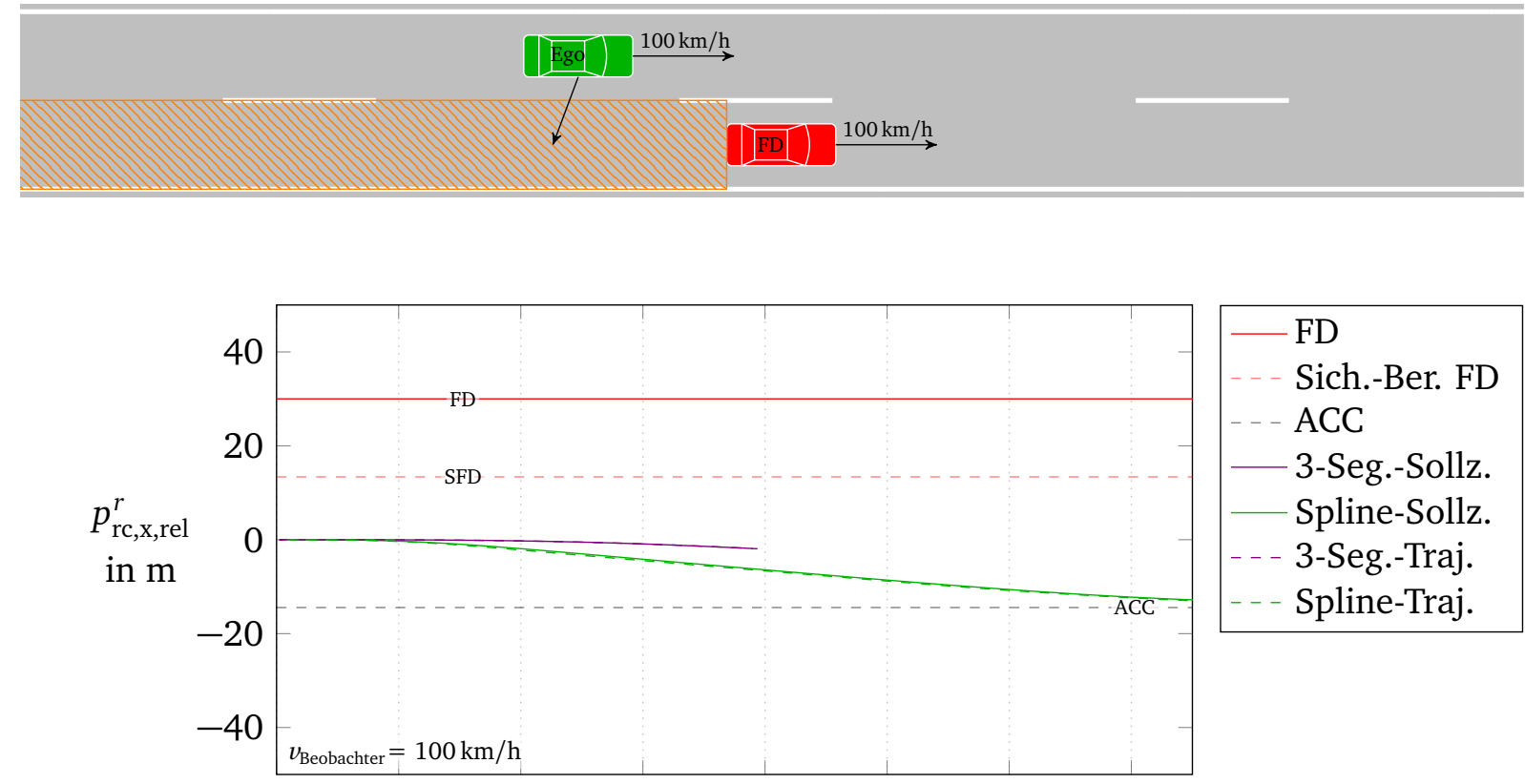

\begin{tabular}{|l}
\hline FD \\
- Sich.-Ber. FD \\
- ACC \\
3-Seg.-Sollz. \\
Spline-Sollz. \\
- - 3-Seg.-Traj. \\
- Spline-Traj.
\end{tabular}


Abbildung C.2: Szenario 2: Ego fährt leicht nach links hinten versetzt hinter FD mit gleicher Geschwindigkeit auf Höhe der Lücke. Ego soll hinter FD den Fahrstreifenwechsel durchführen und ihm folgen.

Das zweiten Szenario ist ähnlich zum ersten, jedoch befindet sich hier das Ego-Fahrzeug schon auf Höhe der Lücke, der Fahrstreifenwechsel kann somit direkt begonnen werden. Wie man sieht, gleichen sich die Quertrajektorien der beiden Planungen sehr (Abbildung C.2). 


\section{Szenario 3}
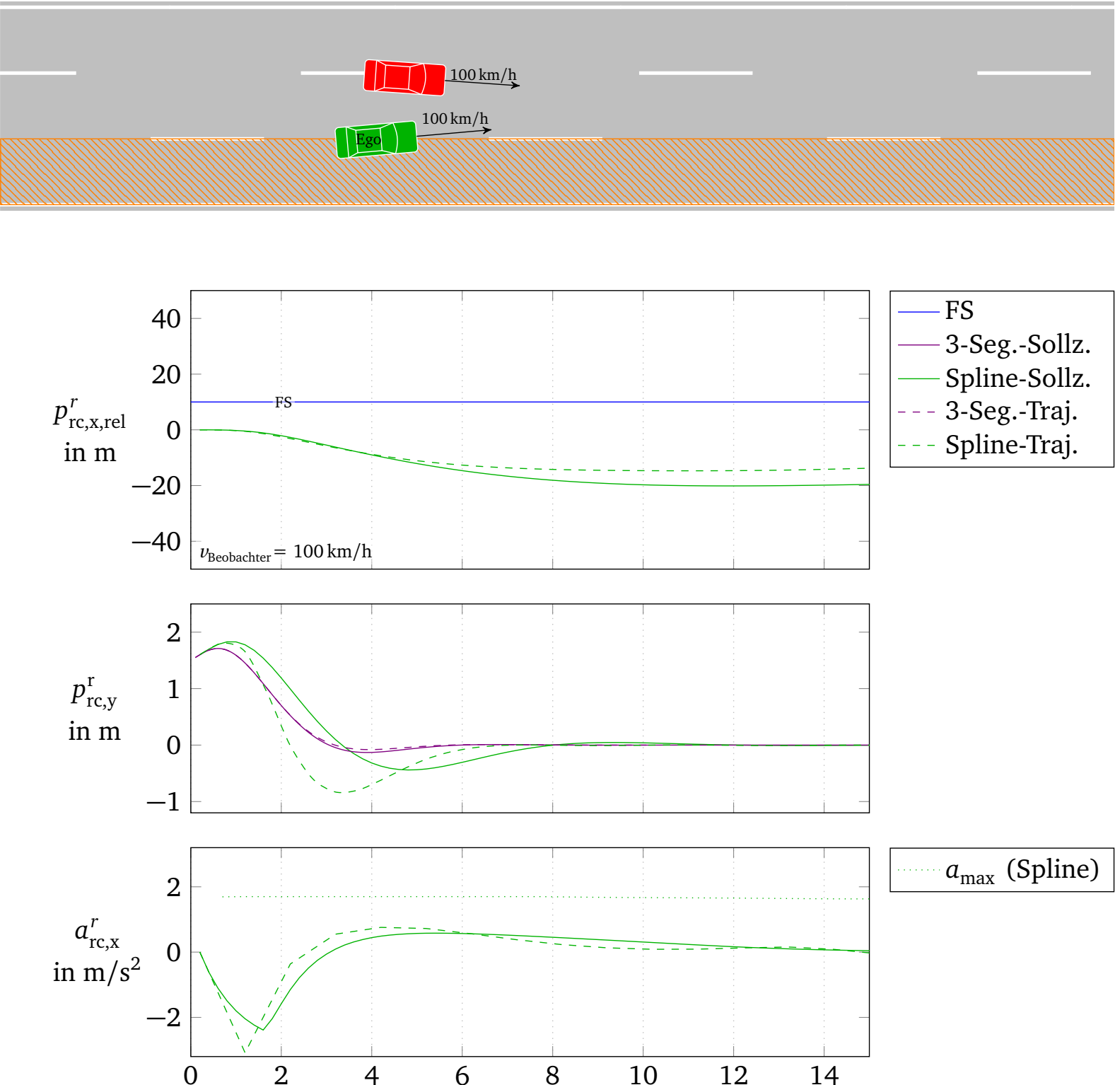

$a_{\max }$ (Spline)



Abbildung C.3: Szenario 3: Ego möchte auf den mittleren Fahrstreifen wechseln, dieser ist jedoch plötzlich belegt. Daher muss der Fahrstreifenwechsel abgebrochen werden. 
In Abbildung C.3 wird ein Abbruch eines Fahrstreifenwechsels betrachtet. Das eigene Fahrzeug befindet sich in einem Fahrstreifenwechsel auf den mittleren Fahrstreifen, während dieser plötzlich von einem anderen Fahrzeug belegt wird.

Die ursprünglich geplante Spline-Trajektorie weist ein etwas stärkeres Überschwingen auf. Dies ist auf die für dieses Szenario relativ lange Segmentlänge von $1 \mathrm{~s}$ zurückzuführen. Durch die zyklische Neuplanung entsteht jedoch ein Sollgrößenverlauf, der nur noch ein sehr kleines Überschwingen aufweist.

Die Drei-Segment-Trajektorie besteht in diesem Szenario nur aus dem 3. Segment. Eine Längstrajektorie wird daher nicht geplant und es ist somit auch keine iterative Optimierung notwendig. 


\section{Szenario 4}

Ziellücke


\begin{tabular}{|l|}
\hline - FD \\
- FS \\
- Sich.-Ber. FD \\
- Sich.-Ber. FS \\
- - ACC \\
3-Seg.-Sollz. \\
- Spline-Sollz. \\
- - 3-Seg.-Traj. \\
\hline- Spline-Traj. \\
\hline
\end{tabular}


$a_{\max }$ (Spline)

Abbildung C.4: Szenario 4: Ego fährt hinter FS und wird demnächst von FD überholt, Ego soll hinter FD den Fahrstreifenwechsel durchführen und ihm folgen.

In diesem Szenario soll das Ego-Fahrzeug auf den linken Fahrstreifen wechseln, auf dem es gerade von einem schnelleren Fahrzeug überholt wird. Die Spline-Planung kann die Längsbeschleunigung schneller aufbauen und beginnt die Querbewegung etwas früher. Dass die Beschleunigung gegen Ende abfällt, obwohl die Sollposition noch nicht erreicht ist, liegt an der Endpunktbewertung. Die Beschleunigung im hinteren Teil hat kaum noch eine Auswirkung auf die Endposition. Somit ist die dargestellte Trajektorie besser im Sinne des Gütekriteriums. In der Praxis ist dies jedoch nicht relevant. Da eine zyklische Neuplanung der Trajektorie stattfindet, verschiebt sich der Endpunkt immer weiter in die Zukunft. Dadurch wird, wie erkennbar ist, die Beschleunigung länger aufrechterhalten. 


\section{Szenario 5}
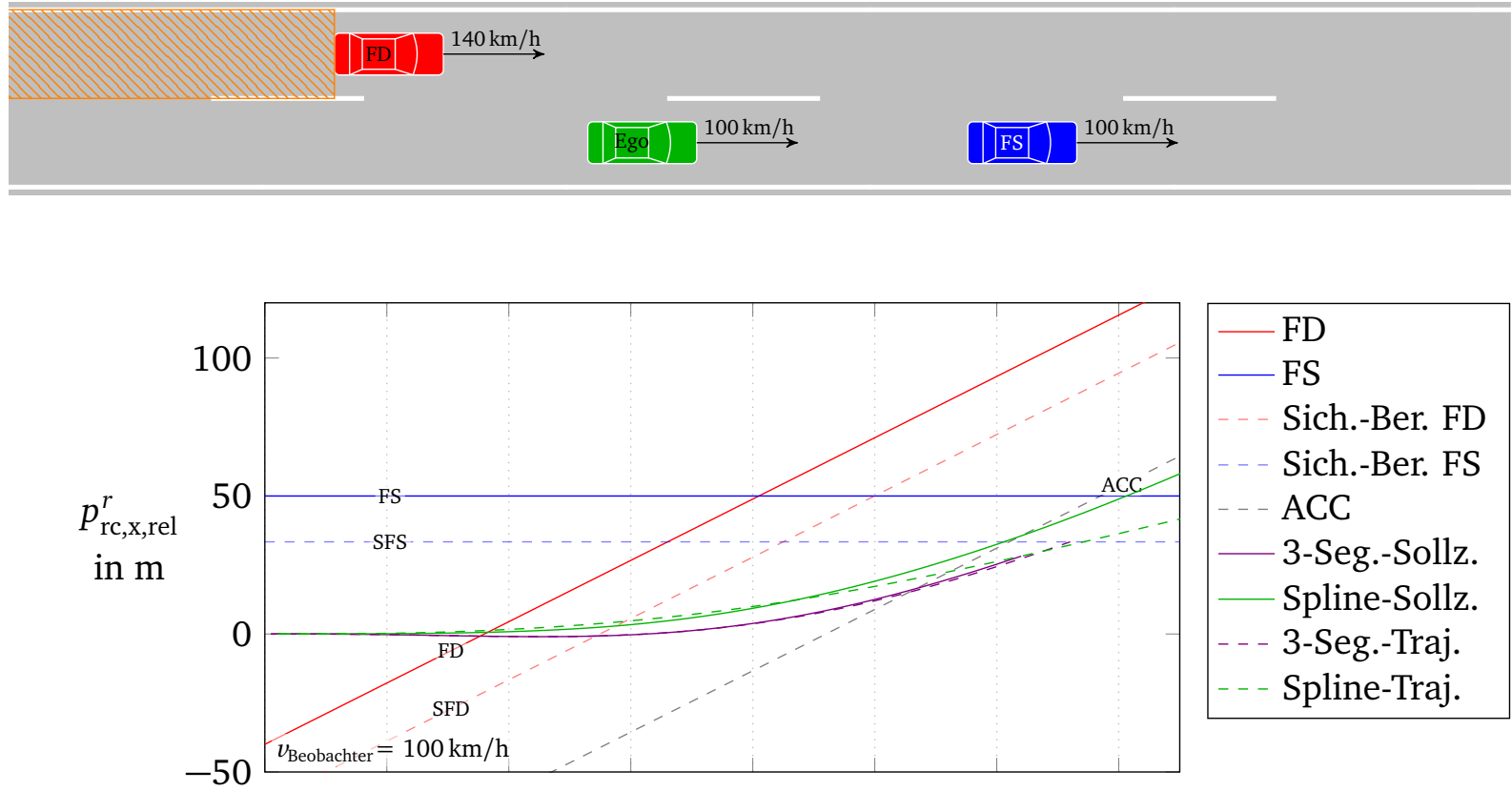

- FD

FS

Sich.-Ber. FD

Sich.-Ber. FS

ACC

3-Seg.-Sollz.

Spline-Sollz.

3-Seg.-Traj.

Spline-Traj.
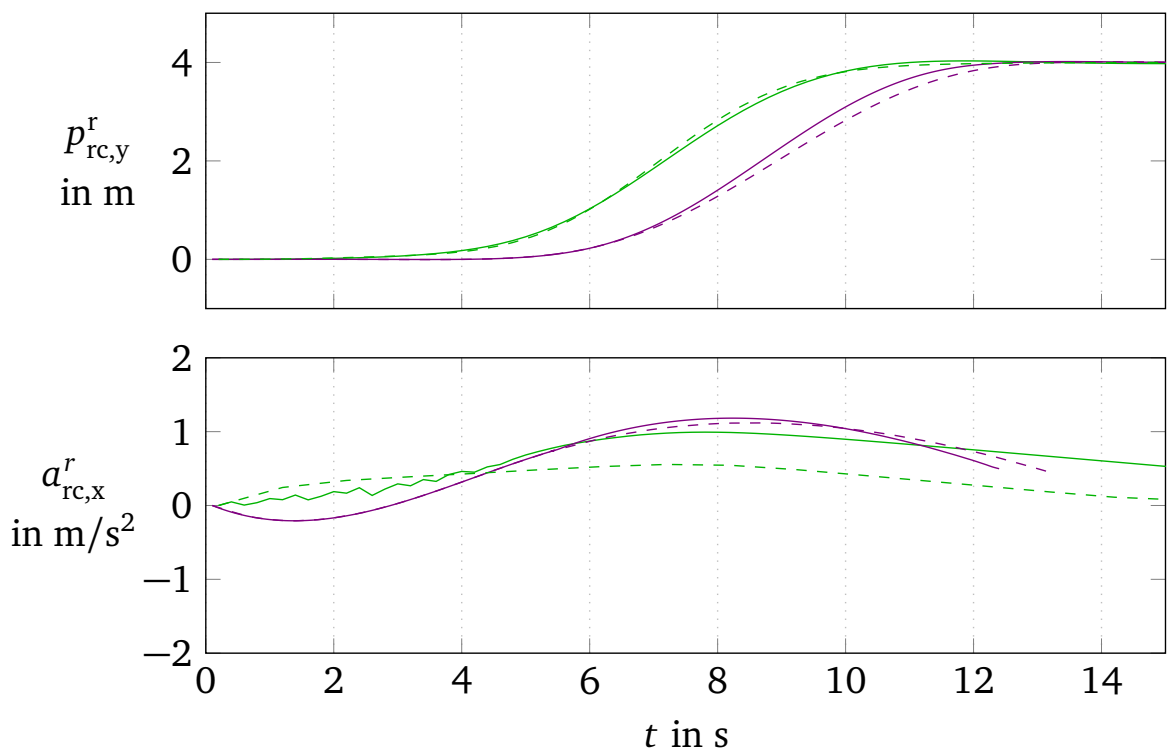

Abbildung C.5: Szenario 5: Ego fährt hinter FS und wird demnächst von FD überholt, Ego soll hinter FD den Fahrstreifenwechsel durchführen und ihm folgen.

Das fünften Szenario entspricht im Wesentlichen dem vierten Szenario, nur dass die Planung hier zu einem früheren Zeitpunkt durchgeführt wird. Es ist deutlich zu erkennen, wie die Spline-Planung den Fahrstreifenwechsel früher beginnt. Die Drei-Segment-Planung versucht hier die Lücke durch eine leichte Verzögerung noch etwas früher zu erreichen. Es wird davon ausgegangen, dass sich dieser Effekt durch Parameteränderungen beseitigen lässt. Es sei jedoch angemerkt, dass derart weit hinten liegende Lücken aktuell aufgrund der Sensorreichweite verworfen werden (Abschnitt 2.4). Das Szenario verdeutlicht allerdings sehr gut den langen Planungshorizont der beiden Planungen. 


\section{Szenario 6}

Ziellücke
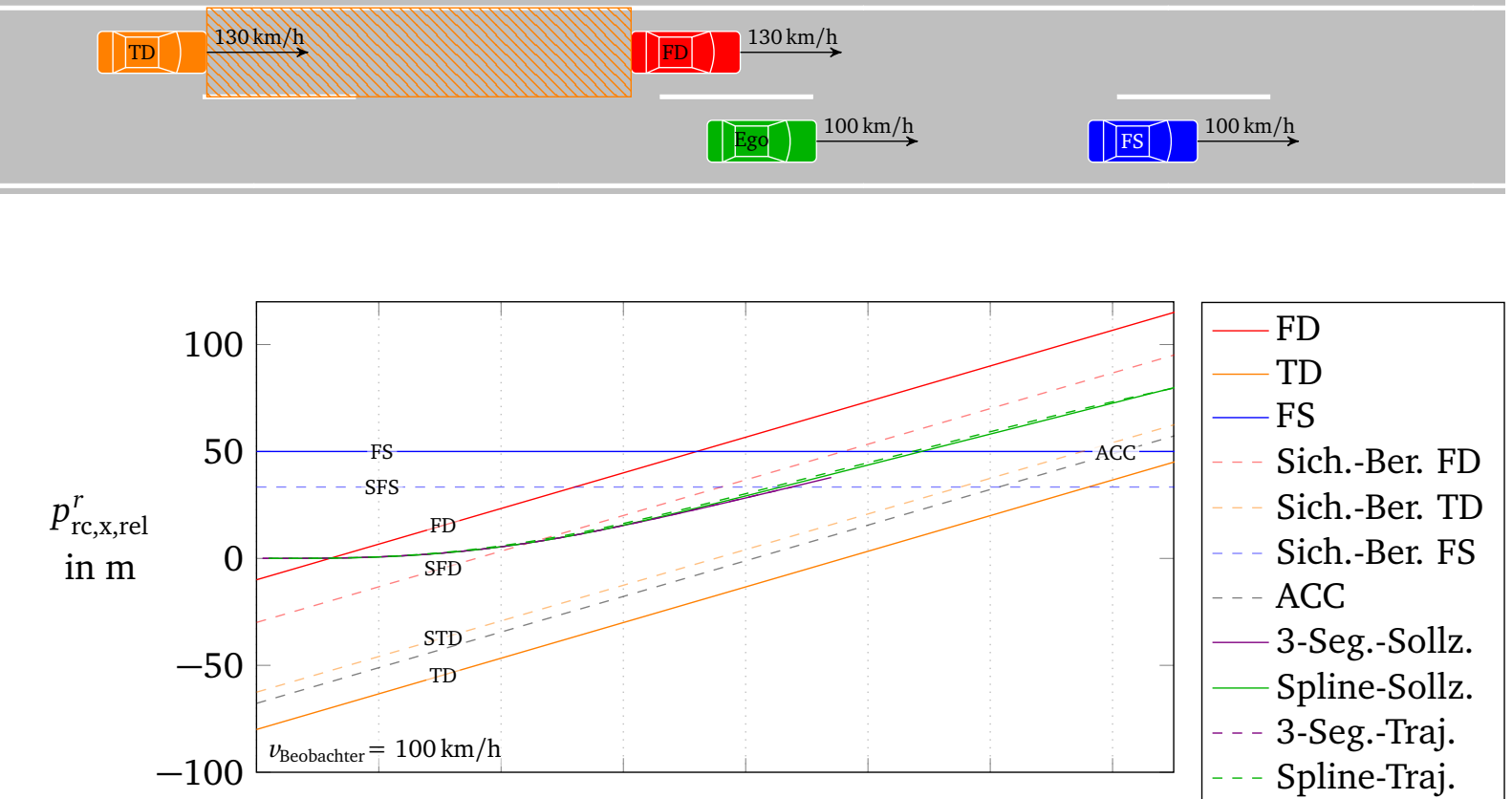

$-\mathrm{FD}$
$-\mathrm{TD}$
$-\mathrm{FS}$

Sich.-Ber. FD

Sich.-Ber. TD

Sich.-Ber. FS

ACC

3-Seg.-Sollz.

Spline-Sollz.

3-Seg.-Traj.

Spline-Traj.
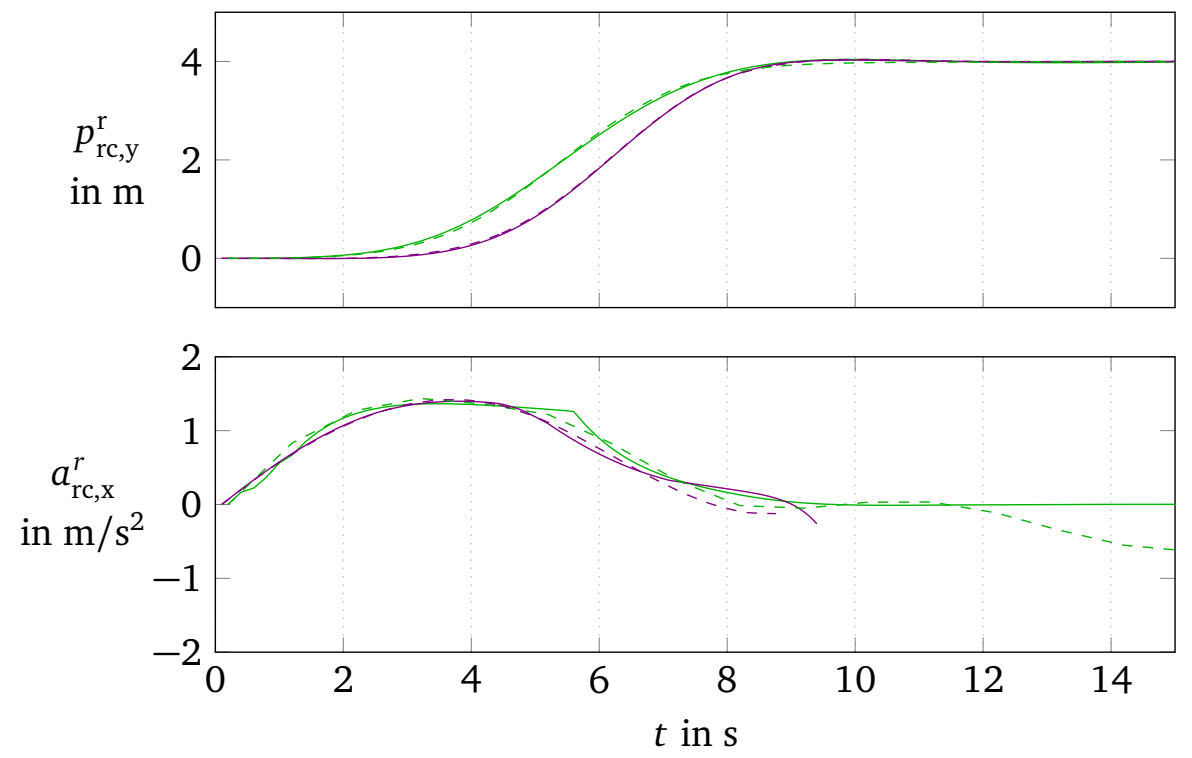

Abbildung C.6: Szenario 6: Ego fährt hinter FS und wird demnächst von FD und TD überholt, Ego soll hinter FD den Fahrstreifenwechsel durchführen und ihm folgen. 
In diesem Szenario soll das Ego-Fahrzeug nach links in eine relativ enge Lücke zwischen zwei schneller fahrenden Fahrzeugen wechseln. Beide Planungen nehmen dabei Rücksicht auf das Fahrzeug TD und beschleunigen stärker als in Szenario 5.

Durch die sehr enge Lücke ist das Ego-Fahrzeug zunächst gezwungen, FD relativ nah zu folgen. Dies führt im Zusammenspiel mit der Endpunktbewertung zu einem Bremsen zwischen $12 \mathrm{~s}$ und $15 \mathrm{~s}$. Durch die zyklische Neuplanung wird die ursprünglich geplante Trajektorie so jedoch nicht bis zum Ende abgefahren. Der ausgegebene Sollgrößenverlauf weist diesen Effekt daher nicht auf. 


\section{Szenario 7}

Ziellücke



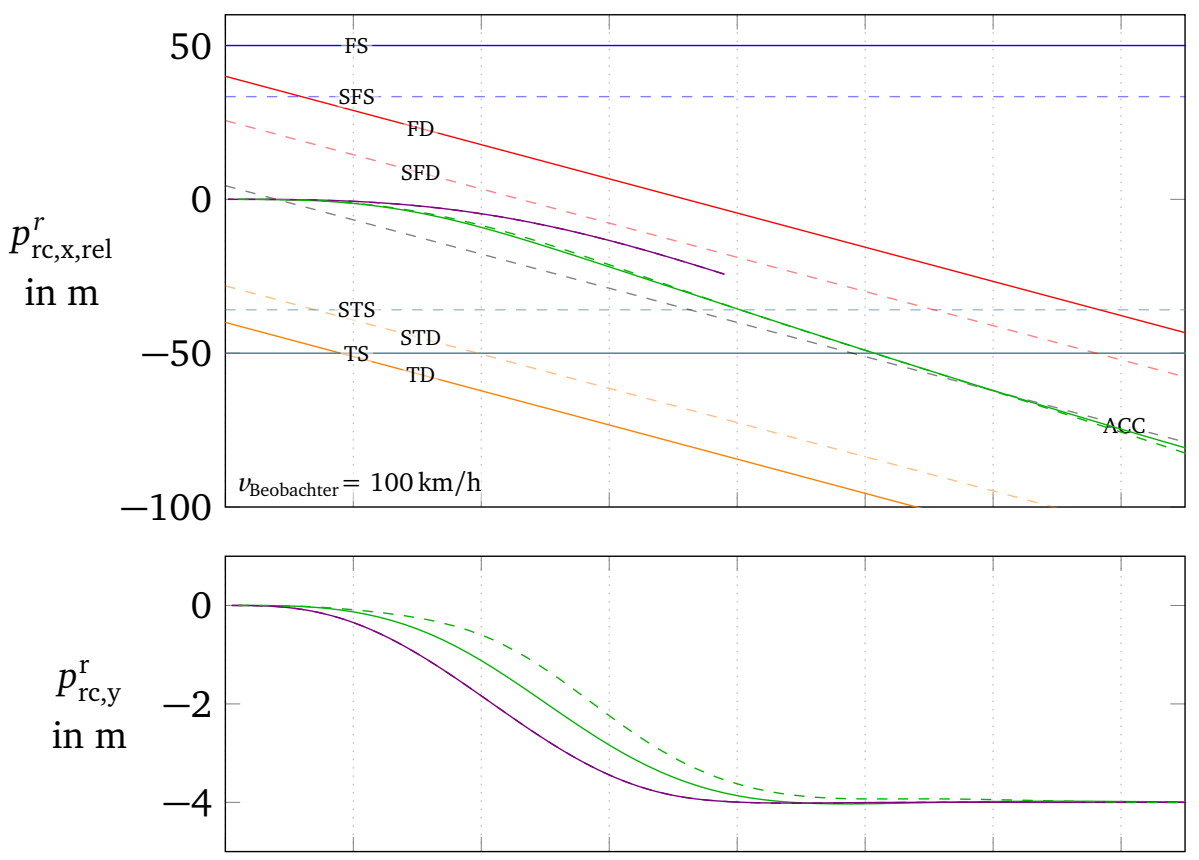

$-\mathrm{FD}$
$-\mathrm{TD}$
$-\mathrm{FS}$
$-\mathrm{TS}$

- Sich.-Ber. FD

Sich.-Ber. TD

Sich.-Ber. FS

Sich.-Ber. TS

ACC

-3-Seg.-Sollz.

Spline-Sollz.

- - - 3-Seg.-Traj.

- - Spline-Traj.



Abbildung C.7: Szenario 7: Ego fährt zwischen zwei Fahrzeugen auf dem linken Fahrstreifen. Ego soll nach rechts zwischen zwei langsamere Fahrzeuge einfädeln.

Im siebten Szenario findet ein Wechsel vom schnellen linken Fahrstreifen in eine Lücke auf dem rechten Fahrstreifen statt. Der Fahrstreifenwechsel wird sehr spät angefordert, das Ego-Fahrzeug befindet sich schon im Soll-Abstand zu FD, fährt jedoch noch mit höherer, noch abzubauender Geschwindigkeit. Die Spline-Planung beginnt den Fahrstreifenwechsel etwas später. Bei beiden Planungen geht der Einfluss auf TS aktuell nicht mit ins Gütekriterium ein. 


\section{Szenario 8}
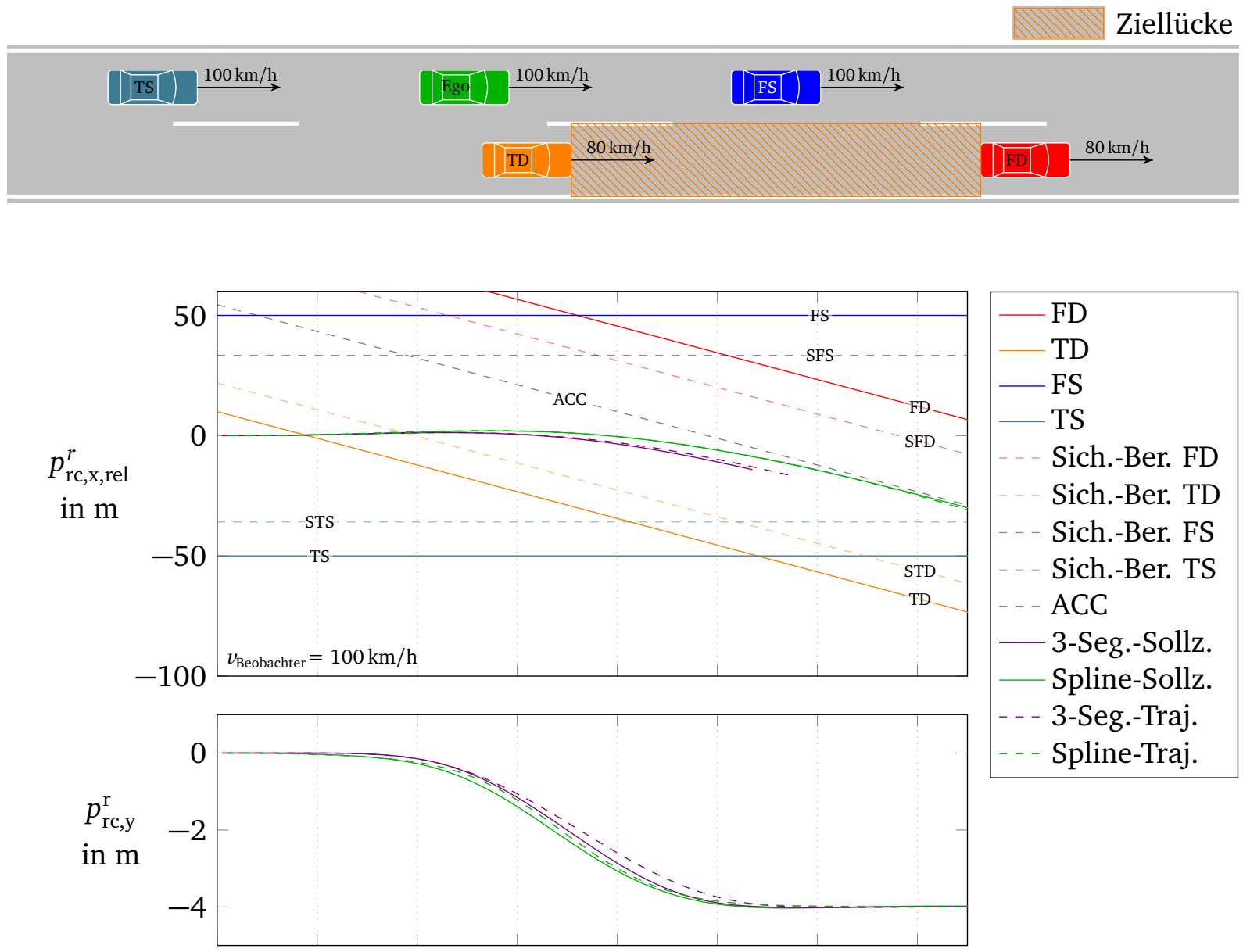

\begin{tabular}{|l|}
\hline - FD \\
- TD \\
- FS \\
\hline TS \\
- Sich.-Ber. FD \\
Sich.-Ber. TD \\
- - Sich.-Ber. FS \\
- Sich.-Ber. TS \\
- ACC \\
- 3-Seg.-Sollz. \\
- Spline-Sollz. \\
-- - 3-Seg.-Traj. \\
--- Spline-Traj. \\
\hline
\end{tabular}

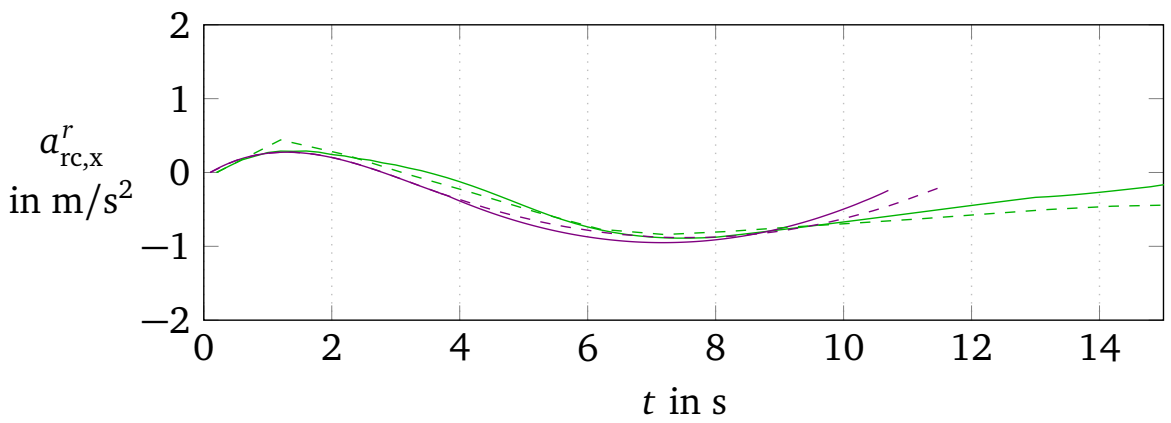

Abbildung C.8: Szenario 8: Ego fährt zwischen zwei Fahrzeugen auf dem linken Fahrstreifen. Ego soll nach rechts zwischen zwei langsamere Fahrzeuge einfädeln. Hierbei wird der Fahrstreifenwechsel frühzeitig angefordert.

Das achte Szenario entspricht dem siebten, wobei der Fahrstreifenwechsel hier vom Fahrer früher angefordert wurde. Der sich ergebene Verlauf unterscheidet sich kaum zwischen den Planungen. Beide beschleunigen noch etwas, um die Lücke schneller zu erreichen. Es ist davon auszugehen, dass sich dieser Effekt, falls er nicht gewünscht ist, durch Berücksichtigung der Verlustenergie vermeiden ließe. Durch die frühzeitige Anforderung und Planung des Fahrstreifenwechsels können die Beschleunigungen wesentlich geringer ausfallen als dies im siebten Szenario der Fall ist. 


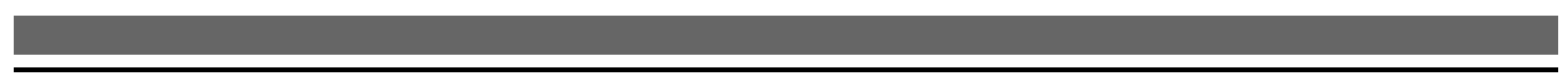




\section{Kritische Szenarien für die Trajektorienplanung}

Es gibt einige Szenarien, in denen die Trajektorienplanung, teilweise auch aufgrund der Umfeldsensorik, an ihre Grenzen stößt. Einige davon werden im Folgenden dargestellt. Dies betrifft im Wesentlichen Situationen, bei denen Umfeldfahrzeuge den Fahrstreifen wechseln. Selbst wenn von der Umfelderkennung eine prädizierte Querbewegung geliefert wird, kann diese in der Drei-Segment-Planung nur sehr schwer berücksichtigt werden.

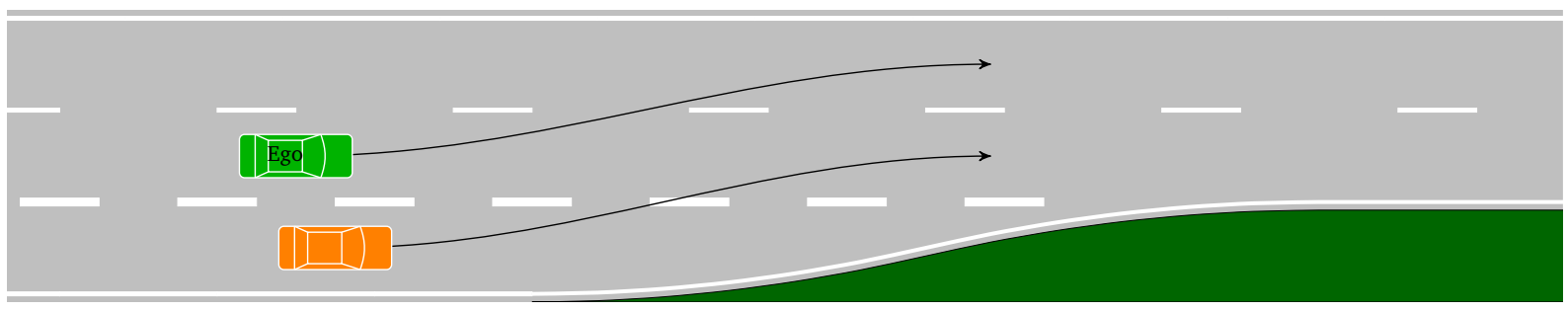

Abbildung D.1: Bei diesem Szenario ist es wichtig zu erkennen, dass das Umfeldfahrzeug den Fahrstreifen wechseln wird. Ist eine prädizierte Querbewegung für das andere Fahrzeug vorhanden, so ließe sich diese in der Spline-Planung verwenden. Für die Drei-Segment-Planung ist dies nicht so einfach umsetzbar, da für die Lückenbetrachtung das Fahrzeug den Fahrstreifen entweder belegt oder nicht.

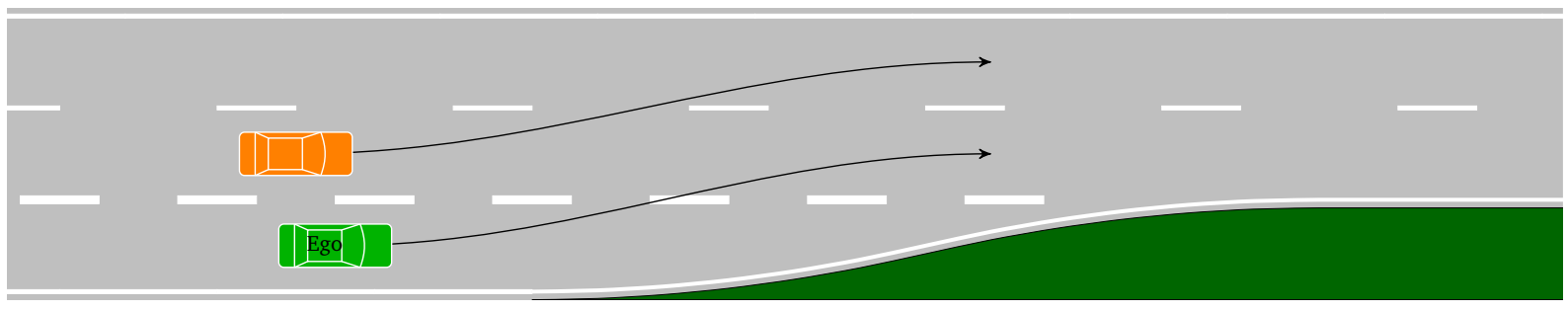

Abbildung D.2: Hier muss zum einen erkannt werden, dass der eigene Fahrstreifen langsam schmaler wird, zum anderen muss erkannt werden, dass der Fahrer auf dem Zielfahrstreifen kooperiert und er demnächst befahrbar sein wird. Diese Verarbeitung ist in die Spline-Planung integrierbar, in die Drei-Segment-Planung schwer bzw. gar nicht. 


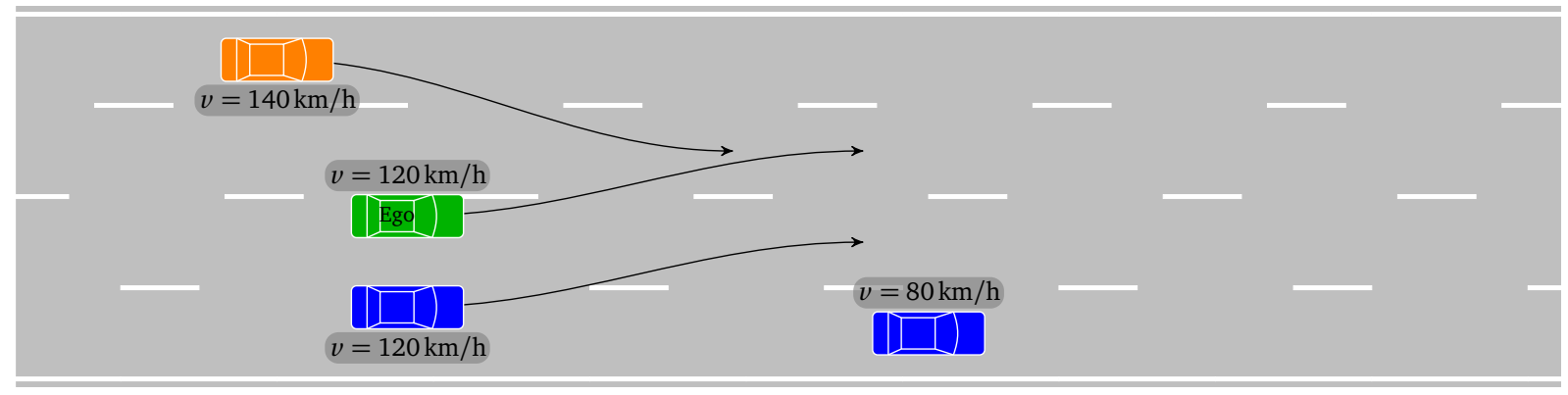

Abbildung D.3: In dieser Situation wechseln die beiden rechten Fahrzeuge jeweils einen Fahrstreifen weiter nach links. Plötzlich wird der Zielfahrstreifen des EgoFahrzeuges durch ein anderes Fahrzeug blockiert. Hier ist es wichtig den Wechsel des herannahenden Fahrzeugs möglichst frühzeitig zu erkennen. Falls dies nicht möglich ist, muss eine geeignete Reservestrategie gefunden werden, da für das Ego-Fahrzeug ein einfacher Wechsel weder auf den Start- noch auf den Zielfahrstreifen möglich ist.

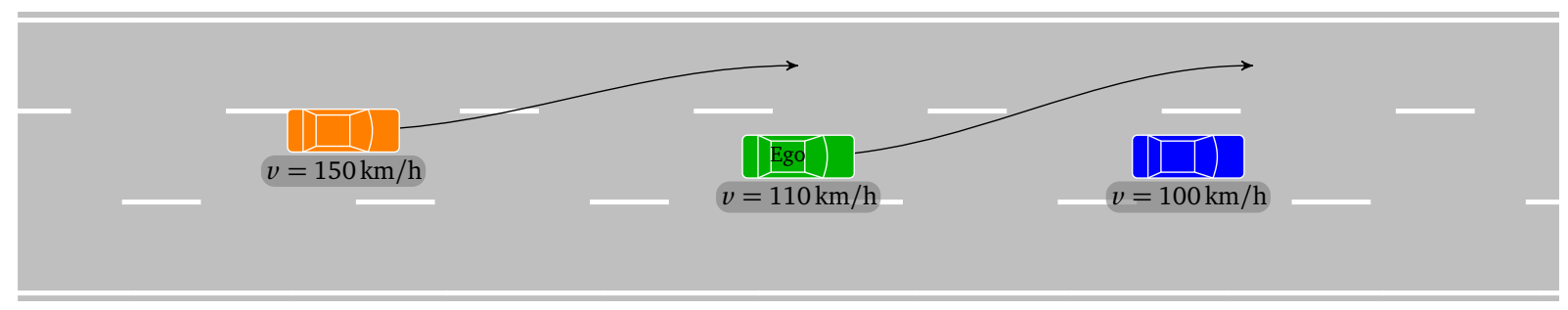

Abbildung D.4: Das rückwärtige Fahrzeug nähert sich mit hoher Geschwindigkeit, hat den Blinker nach links gesetzt und ist darauf angewiesen, den Fahrstreifenwechsel durchführen zu können. Das Ego-Fahrzeug darf einen Fahrstreifenwechsel in dieser Situation nicht starten, auch wenn der Zielfahrstreifen zum Startzeitpunkt komplett frei ist.



Abbildung D.5: Das Ego-Fahrzeug möchte aufgrund eines Geschwindigkeitsüberschusses den Fahrstreifen wechseln. Auf den Zielfahrstreifen wechselt jedoch noch ein anderes Fahrzeug. Herausforderung ist auch hier, diesen Fahrstreifenwechsel frühzeitig zu erkennen. 


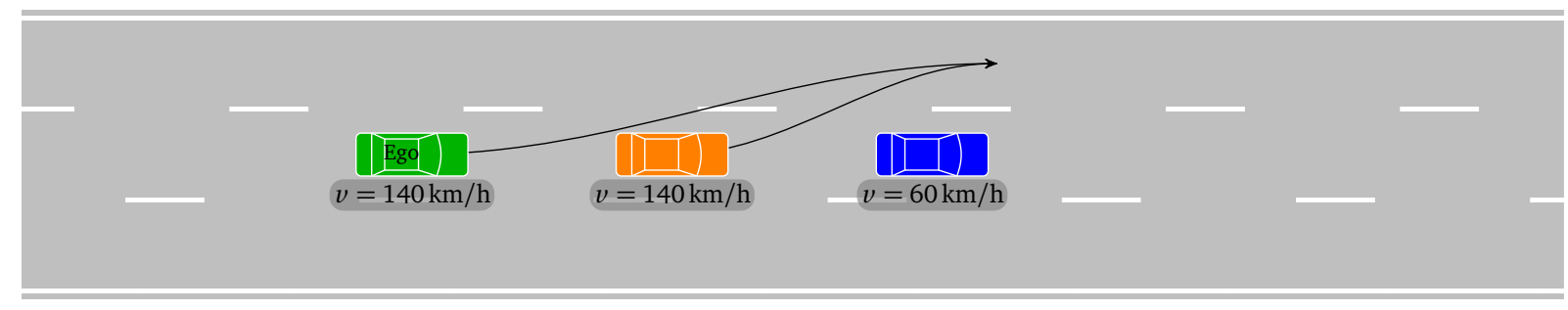

Abbildung D.6: Vor dem Ego-Fahrzeug fährt ein anderes Fahrzeug mit etwa gleicher Geschwindigkeit. Dieses wechselt kurz vor einem sehr viel langsameren Fahrzeug den Fahrstreifen. Das Ego-Fahrzeug wechselt entweder schnell den Fahrstreifen oder bremst rechtzeitig ab. Es wäre hier wünschenswert, das langsame Fahrzeug frühzeitig erkennen zu können.

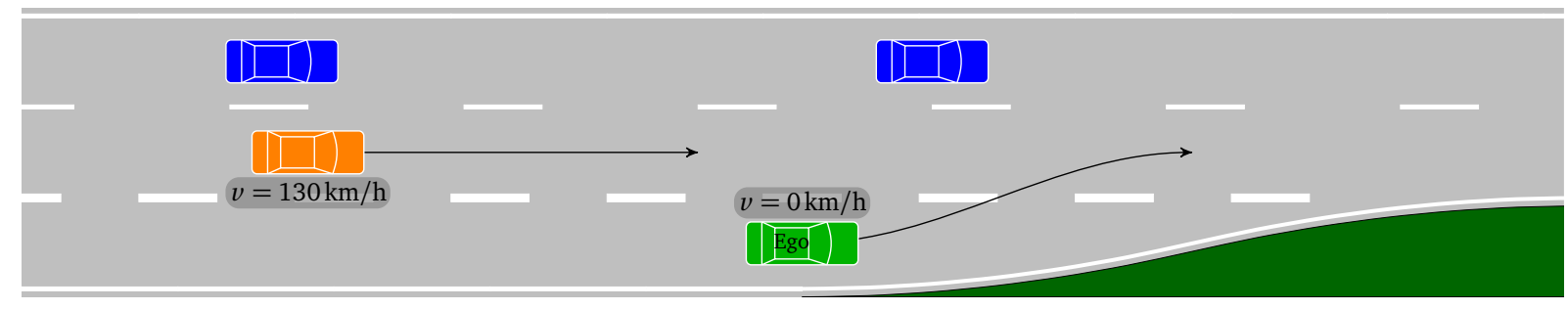

Abbildung D.7: Das Fahrzeug befindet sich stehend auf dem Beschleunigungsstreifen und muss auf die Autobahn wechseln. Für die Trajektorienplanung ist dies eine eher einfache Aufgabe, umso schwerer ist sie jedoch für die Umfelderfassung. Die Geschwindigkeitsdifferenzen sind sehr hoch, und damit auch die erforderlichen Sensorreichweiten. 







\section{E Optimierung mit Nebenbedingungen}

Ziel ist die Optimierung eines Gütekriteriums in der Form

$$
J=\underline{\mathbf{x}}^{\mathrm{T}} \underline{\mathbf{W}}_{1} \underline{\mathbf{x}}+\underline{\mathbf{w}}_{2}^{\mathrm{T}} \underline{\mathbf{x}}+w_{3}
$$

mit einer symmetrischen Matrix $\underline{\mathbf{W}}_{1}$ unter der Nebenbedingung

$$
\underline{\mathbf{C}} \cdot \underline{\mathbf{x}}=\underline{\mathbf{d}} .
$$

Die Nebenbedingung wird zur Gütefunktion mit Hilfe eines Lagrange-Multiplikators addiert [Bronstein, 2001, S. 418 f.]:

$$
J=\underline{\mathbf{x}}^{\mathrm{T}} \underline{\mathbf{W}}_{1} \underline{\mathbf{x}}+\underline{\mathbf{w}}_{2}^{\mathrm{T}} \underline{\mathbf{x}}+w_{3}+\underline{\lambda}^{\mathrm{T}}(\underline{\mathbf{C}} \cdot \underline{\mathbf{x}}-\underline{\mathbf{d}})
$$

Damit $J$ minimal wird, müssen die Ableitungen nach $\underline{\mathbf{x}}$ und $\underline{\lambda}$ Null sein:

$$
\begin{aligned}
& \left(\frac{\partial}{\partial \underline{\mathbf{x}}} J\right)^{\mathrm{T}}=2 \cdot \underline{\mathbf{w}}_{1}^{\mathrm{T}} \underline{\mathbf{x}}+\underline{\mathbf{w}}_{2}+\underline{\mathbf{C}}^{\mathrm{T}} \underline{\boldsymbol{\lambda}}=\underline{\mathbf{0}} \\
& \left(\frac{\partial}{\partial \underline{\lambda}} J\right)^{\mathrm{T}}=\underline{\mathbf{C}} \cdot \underline{\mathbf{x}}-\underline{\mathbf{d}}=\underline{\mathbf{0}}
\end{aligned}
$$

In Matrixform ergibt sich:

$$
\left[\begin{array}{cc}
2 \cdot \underline{\mathbf{W}}_{1}^{\mathrm{T}} & \underline{\mathbf{C}}^{\mathrm{T}} \\
\underline{\mathbf{C}} & \underline{\mathbf{0}}
\end{array}\right] \cdot\left[\begin{array}{l}
\underline{\mathbf{x}} \\
\underline{\lambda}
\end{array}\right]=\left[\begin{array}{c}
-\underline{\mathbf{w}}_{2} \\
\underline{\mathbf{d}}
\end{array}\right]
$$

Dieses lineare Gleichungssystem kann dann leicht gelöst werden. 


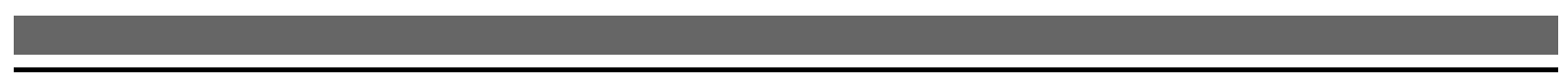




\section{F Der Versuchsträger}

In diesem Kapitel wird auf einige Details des Versuchsträgers eingegangen. Zunächst wird der strukturelle Aufbau des Versuchsträgers dargestellt. Anschließend wird die Identifikation und Regelung der Längsaktorik vorgestellt. Abschließend wird die Filterung der Umfeldinformationen beschrieben.

\section{F.1 Struktur}

Die im Versuchsträger notwendigen Berechnungen finden auf zwei verschiedenen Plattformen statt. Auf der Echtzeithardware (Vector VN8910) werden alle $20 \mathrm{~ms}$ die Kameradaten ausgewertet und zusammen mit der üblichen Fahrzeugsensorik das Straßenkoordinatensystem erzeugt. Weiterhin wird die Trajektorie abgetastet und der Soll-Zustand daraus dem Regler zugeführt. Längs- und Querregler berechnen aus der Soll-Ist-Differenz das nötige Lenkmoment sowie die anzufordernde Beschleunigung.

Asynchron dazu wird auf dem Messrechner innerhalb der Software CANape die Transformation des Umfelds sowie die Trajektorienplanung berechnet. Dies findet etwa alle $100 \mathrm{~ms}$ statt (Bei der Spline Planung alle $200 \mathrm{~ms}$ ). Um zu vermeiden, dass es durch die asynchrone Berechnung zu Problemen kommt, sind alle Signale mit Zeitstempeln versehen. In Abbildung F.1 ist eine vereinfachte Systemstruktur des Versuchsträgers dargestellt.

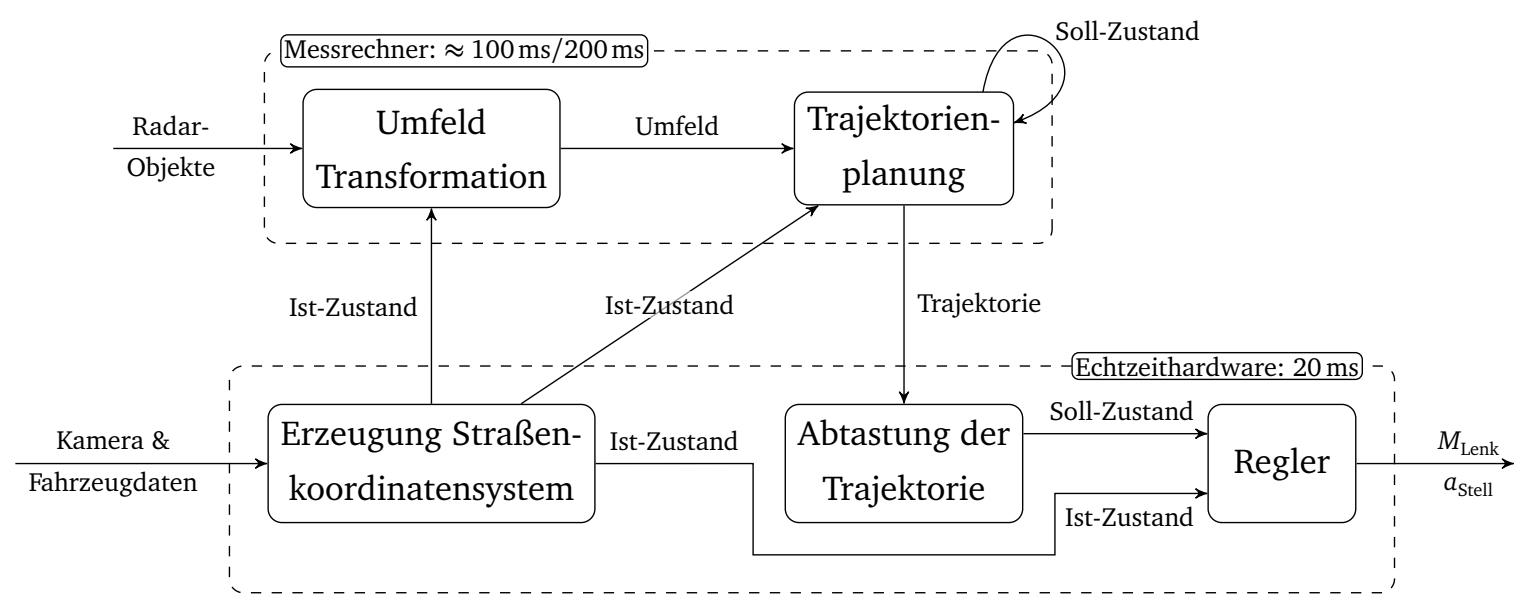

Abbildung F.1: Vereinfachte Systemstruktur des Versuchsträgers 


\section{F.2 Regelung}

Die Regelung von Längs- und Querrichtung geschieht in diesem Projekt weitestgehend unabhängig voneinander. Der Querregler wurde vom Vorgängerprojekt übernommen. Für die Längsrichtung wurde die Aktorik zunächst identifiziert und anschließend ein Trajektorienfolgeregler ausgelegt.

\section{F.2.1 Identifikation der Längsaktorik}

Zur Bestimmung der Maximalgrenzen für die Trajektorienplanung und zur Entwicklung der Längsregelung wurde die Längsaktorik identifiziert.

\section{Maximalgrenzen}

Zur Bestimmung der Maximalbeschleunigung wurde das Fahrzeug auf einer Teststrecke aus dem Stand beschleunigt. Die vorgegebene Beschleunigung lag bei den Fahrversuchen weit über den Fahrzeugmöglichkeiten. Die Messungen wurden sowohl in der Getriebestellung „D“ als auch in „S“ vorgenommen. Anschließend wurde auch eine Messung der Beschleunigung aus einer Fahrt mit $100 \mathrm{~km} / \mathrm{h}$ durchgeführt. Es fiel dabei auf, dass das Getriebe vom fünften Gang nicht herunterschaltet und die maximal mögliche Beschleunigung deutlich niedriger ausfällt. Die Maximalverzögerung wurde auf ähnliche Art und Weise bestimmt (Abbildung F.2).



Abbildung F.2: Messreihen zur Bestimmung der Maximalbeschleunigung und -verzögerung

Ergebnis der Identifikation ist eine Maximalbeschleunigung, die sich aus der begrenzten Motorleistung und den Fahrwiderständen unter Berücksichtigung eines Sicherheitsfaktors ergibt. Zusätzlich ist die Maximalbeschleunigung auf $1,8 \mathrm{~m} / \mathrm{s}^{2}$ begrenzt. Die maximale Verzögerung wird mit $3,3 \mathrm{~m} / \mathrm{s}^{2}$ modelliert. 


\section{Dynamisches Verhalten}

Neben den Grenzen für minimale und maximale Beschleunigung wurde auch die Dynamik des Systems untersucht. Zur Untersuchung wurden Sprungantworten für unterschiedliche Sprunghöhen aufgenommen. Hierbei fällt auf, dass das Systemverhalten im beschleunigenden Bereich anders als im verzögernden Bereich ist. Das System braucht länger um eine Beschleunigung aufzubauen, als um eine Verzögerung abzubauen (Abbildung F.3).
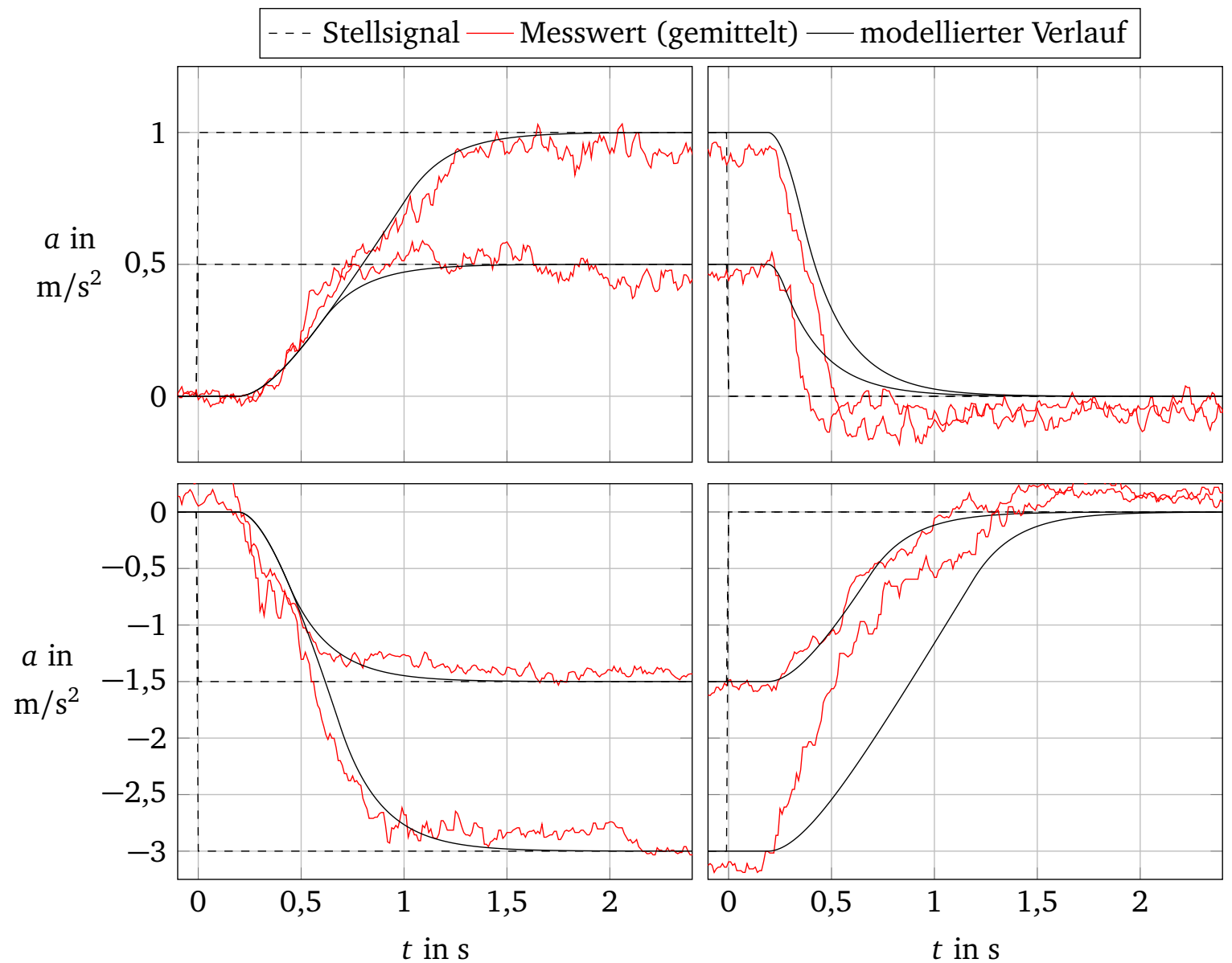

Abbildung F.3: Sprungantworten der Längsaktorik auf steigende und fallende Sprünge. Deutlich zu erkennen die Ratenbegrenzung, sowie deren unterschiedliche Grenzwerte für positive und negative Werte

Zur Modellierung der Längsaktorik wurde ein System mit Totzeit, Ratenbegrenzung, Sättigung und $\mathrm{PT}_{1}$-Verhalten gewählt (Abbildung F.4). Die zugehörigen Parameter befinden sich in Tabelle F.1.

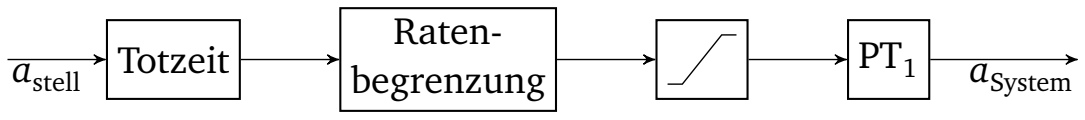

Abbildung F.4: Modell der Längsaktorikschnittstelle

Das System kam innerhalb der CarMaker-Simulation zum Einsatz, die Parametrierung des Längsreglers geschah direkt am realen System. 
Tabelle F.1: Modellparameter der Längsaktorikschnittstelle

\begin{tabular}{lc} 
Größe & Wert \\
\hline Totzeit & $0,2 \mathrm{~s}$ \\
Zeitkonstante des $\mathrm{PT}_{1}$-Gliedes & $0,2 \mathrm{~s}$ \\
steigende Ratenbegrenzung für $\mathrm{a}>0$ & $1,2 \mathrm{~m} / \mathrm{s}^{3}$ \\
steigende Ratenbegrenzung für $\mathrm{a}<0$ & $3 \mathrm{~m} / \mathrm{s}^{3}$ \\
fallende Ratenbegrenzung & $-6 \mathrm{~m} / \mathrm{s}^{3}$
\end{tabular}

\section{F.2.2 Längsregler}

Wie in Abschnitt F.2.1 ersichtlich ist, wird die vorgegebene Sollbeschleunigung im stationären Fall gut eingeregelt. Zum exakten Abfahren der Trajektorie ist jedoch eine Positionsregelung notwendig. Als Trajektorienfolgeregler wird ein Zustandsregler verwendet (Abbildung F.5). Das dynamische Verhalten von Motor und Antrieb soll durch den Zustandsregler möglichst nicht beeinflusst werden. Vielmehr ist es Aufgabe des Reglers, ein Auseinanderdriften der Soll- und Istgrößen für Position und Geschwindigkeit zu verhindern. Der Aufbau des Zustandsreglers entspricht daher dem eines einfachen Reglers für ein Massepunktmodell mit Eingang Beschleunigung.

Die Parameter des Zustandsreglers wurden in Fahrversuchen so ermittelt, dass ein guter Kompromiss zwischen Regelgüte und Komfort gefunden werden konnte. Regelabweichungen treten in den Fahrversuchen im Wesentlichen nur dann auf, wenn die Aktorik die geforderte Beschleunigung nicht realisieren kann. Ursache dafür kann eine nicht berücksichtigte Steigung der Straße oder eine falsche Gangwahl sein.

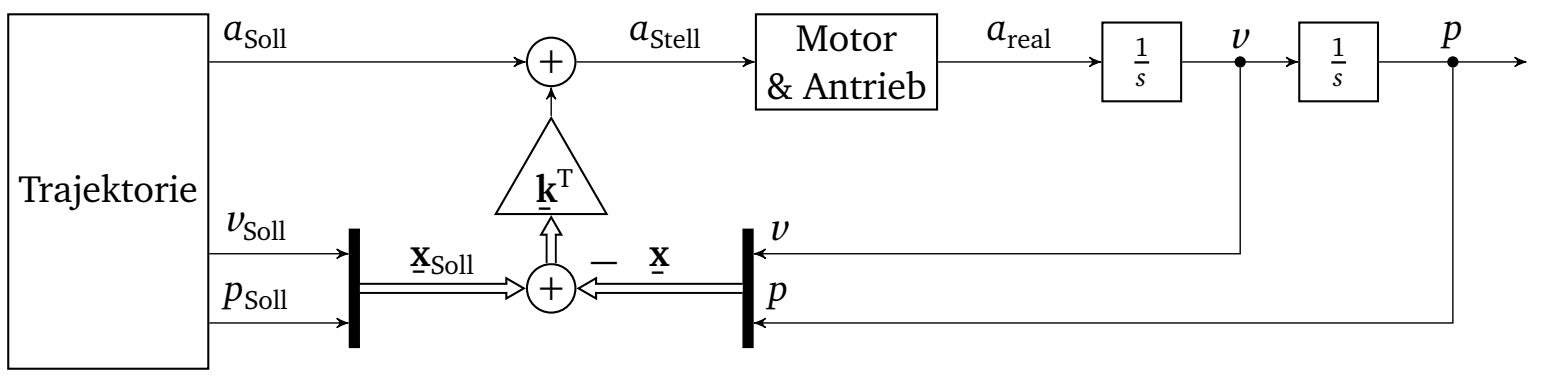

Abbildung F.5: Trajektorienfolgeregler für die Längsrichtung

Folgende Reglerparameter wurden während der Versuchsfahrten verwendet:

$$
\underline{\mathbf{k}}^{\mathrm{T}}=\left[\begin{array}{ll}
k_{\mathrm{LR}, \mathrm{v}} & k_{\mathrm{LR}, \mathrm{p}}
\end{array}\right] \quad k_{\mathrm{LR}, \mathrm{v}}=0,7 / \mathrm{s} \quad k_{\mathrm{LR}, \mathrm{p}}=0,3 / \mathrm{s}^{2}
$$

Abbildung F.6 zeigt die Soll- und Istgrößen, sowie die Positionsabweichung bei einem simulierten Beschleunigungsvorgang von $80 \mathrm{~km} / \mathrm{h}$ auf $100 \mathrm{~km} / \mathrm{h}$. Verwendet wurde das in Abschnitt F.2.1 identifizierte Modell. Als Trajektorie wurde ein Positionsverlauf in Form eines 
Polynoms fünften Grades verwendet. Wie man erkennen kann, bleibt die Positionsabweichung dabei unter 0,5 m. Dies ist bei den üblichen Abständen auf der Autobahn nicht von Bedeutung.


Abbildung F.6: Folgeverhalten des geregelten Längssystems bei einem simulierten Beschleunigungsvorgang von $80 \mathrm{~km} / \mathrm{h}$ auf $100 \mathrm{~km} / \mathrm{h}$ in Form eines Polynoms fünften Grades

\section{F.3 Filterung der Fahrstreifenbelegungswahrscheinlichkeiten}

Während der Entwicklung hat sich herausgestellt, dass die Zuordnung der Umfeldfahrzeuge zu den Fahrstreifen durch vorgelagerte Module nicht zufriedenstellend war. Obwohl dies nicht als Teil des Projektes vorgesehen war, wurde versucht, das Problem einzudämmen, um sinnvolle Versuchsfahrten zu ermöglichen. Bei der Untersuchung sind zwei wesentliche Probleme zu Tage getreten:

1. Durch kurzfristige Messfehler werden Fahrzeuge einem anderen Fahrstreifen zugeordnet.

2. Bei weit entfernten Fahrzeugen ist die geschätzte Varianz der Querposition sehr groß. Dadurch sinkt die Belegungswahrscheinlichkeit für einen einzelnen Fahrstreifen leicht unter den Schwellwert. Das Fahrzeug wird dann keinem Fahrstreifen zugeordnet und somit ignoriert.

Um die Leistungsfähigkeit zu verbessern, wurde ein Konzept bestehend aus einer Filterung und einer nachgeschalteten Logik entwickelt (Abbildung F.7).

Zunächst wird ausgenutzt, dass die Fahrzeuge den Fahrstreifen nur vergleichsweise selten wechseln. Als einfache Lösung wird dazu ein Tiefpassfilter mit einer Zeitkonstante $T=$ $0,5 \mathrm{~s}$ auf die Fahrstreifenbelegungswahrscheinlichkeiten angewendet. Das zweite Problem wird durch eine Logik adressiert, die einen Fahrstreifen, unter der Bedingung, dass das 
Vorher

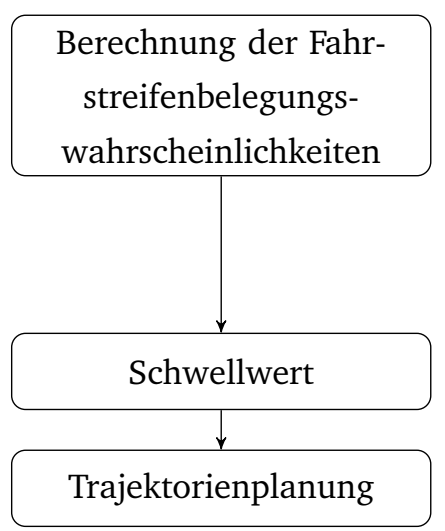

Jetzt

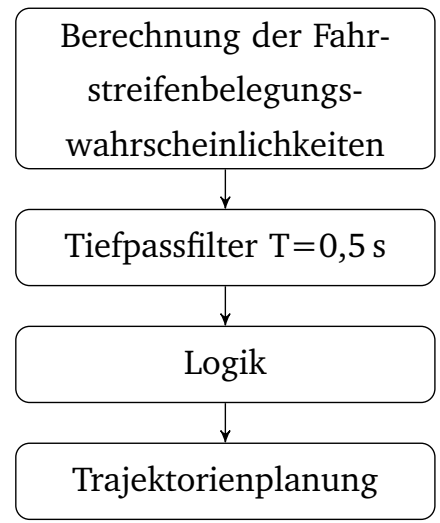

Abbildung F.7: Konzept zur Filterung der Fahrstreifenbelegungswahrscheinlichkeiten im Vergleich zur vorherigen Umsetzung

Existenzmaß $p_{\text {Exist }}$ eines Objektes mindestens 0,95 beträgt, auch dann als belegt ansieht, wenn die Fahrstreifenbelegungswahrscheinlichkeit $p_{\text {Lane }}$ sich zwischen 0,6 und 0,9 befindet und es sich um den am wahrscheinlichsten belegten Fahrstreifen handelt (Abbildung F.8). Das Fahrzeug wird also innerhalb bestimmter Grenzen dem Fahrstreifen mit der höchsten Belegungswahrscheinlichkeit zugeordnet.

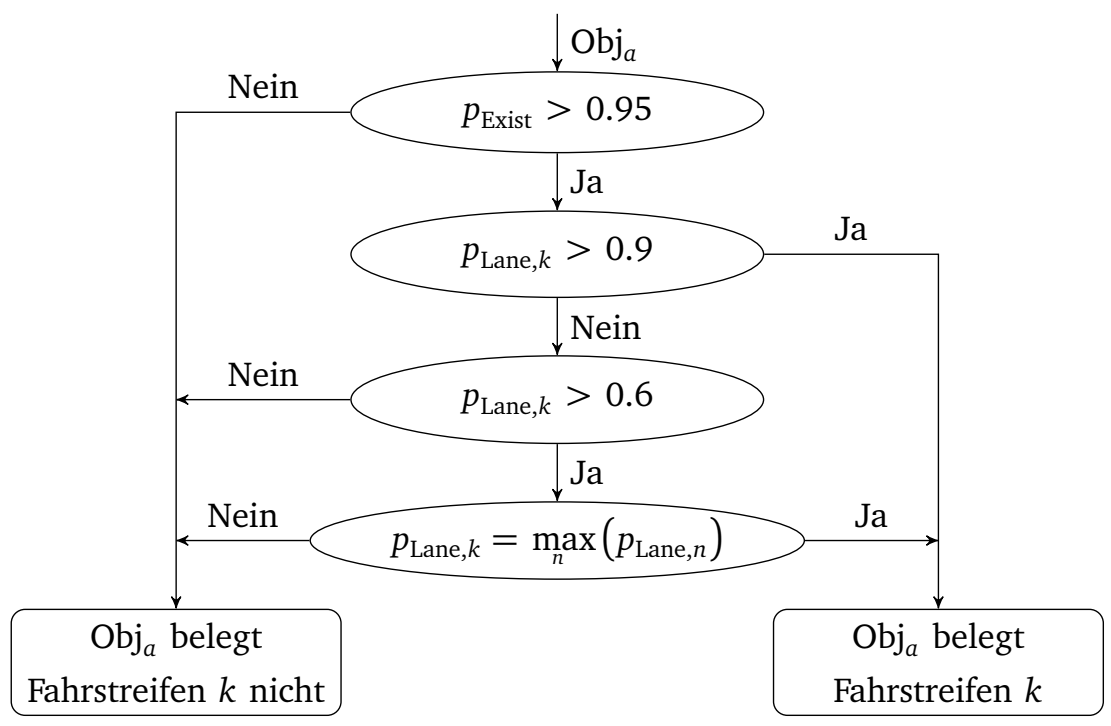

Abbildung F.8: Logik, mit der entschieden wird, ob ein Objekt einen Fahrstreifen belegt oder nicht

Im Rahmen der weiteren Erprobung hat sich eine deutliche Verbesserung ergeben. 


\section{Literaturverzeichnis}

[Bellman, 1957]

[Bewersdorff, 2013]

[Bronstein, 2001]

[Broyden, 1970]

[Dickmanns LC]

[Dickmanns, 1994]

[Dickmanns, 2007]

[DIN ISO 8855]

[Fletcher, 1970]

[Föllinger, 2016]

[Goldfarb, 1970]
R. Bellman: Dynamic Programming. Princeton University Press, 1957.

J. Bewersdorff: Algebra für Einsteiger : Von der Gleichungsauflösung zur Galois-Theorie. 5., erw. Aufl. Wiesbaden: Springer Spektrum, 2013. ISBN: 978-3-658-02261-7. DOI: 10. 1007/978-3-658-02262-4.

I. N. Bronstein, K. A. Semendjajew, G. Musiol und H. Mühlig: Taschenbuch der Mathematik. 5., überarb. und erw. Aufl. Frankfurt am Main: Verlag Harri Deutsch, 2001. ISBN: 3-8171-2005-2.

C. G. Broyden: The Convergence of a Class of Double-rank Minimization Algorithms 1. General Considerations. In: IMA Journal of Applied Mathematics, 6(1), März 1970, S. 76-90. DOI: 10.1093/imamat/6. 1.76 .

E. D. Dickmanns: Lane change (on a free road) since 1987. URL: http: // www . dyna - vision . de /main / A . 3 . 4\%20Lane\%20change . html (abgerufen am 02.05.2017).

E. D. Dickmanns, R. Behringer, D. Dickmanns, T. Hildebrandt, M. Maurer u.a.: The seeing passenger car 'VaMoRs-P'. In: Proceedings of the Intelligent Vehicles '94 Symposium. Paris, Okt. 1994, S. 68-73. DoI: 10.1109/IVS. 1994.639472.

E. D. Dickmanns: Dynamic Vision for Perception and Control of Motion. 1. Aufl. London: Springer-Verlag, 2007. ISBN: 978-1-84628-637-7. DOI: 10. $1007 / 978-1-84628-638-4$.

DIN ISO 8855:2011 Straßenfahrzeuge - Fahrzeugdynamik und Fahrverhalten - Begriffe. Deutsches Institut für Normung e. V., Nov. 2013.

R. Fletcher: A new approach to variable metric algorithms. In: The Computer Journal, 13(3), 1970, S. 317-322. DoI: 10.1093/comjnl/ 13.3.317.

O. Föllinger, U. Konigorski, B. Lohmann, G. Roppenecker und A. Trächtler: Regelungstechnik : Einführung in die Methoden und ihre Anwendung. 12., überarbeitete Auflage. Berlin: VDE-Verlag, 2016. IsBn: 978-3-8007-4201-1.

D. Goldfarb: A Family of Variable-Metric Methods Derived by Variational Means. In: Mathematics of computation, 24(109), 1970, S. 23-26. Dor: 10. 1090/S0025-5718-1970-0258249-6. 
[Graichen, 2017]

[Gundlach, 2017]

[Hafner, 2016]

[Hansen, 2016a]

[Hansen, 2016b]

[Hansen, 2017]

[Katke, 2014]

[Lagarias, 1998]

[Nelder, 1965]

[Nilsson, 2015]
K. Graichen: Vorlesungsskript: Methoden der Optimierung und optimalen Steuerung WS 2017 / 2018. 2017. URL: https : / / www . uni-ulm . de/in/mrm/lehre/vorlesungen - wintersemester/methoden - der optimierung-u-optimalen-steuerung/ (abgerufen am 24. 10. 2017).

I. Gundlach, U. Konigorski und J. Hoedt: Zeitoptimale Trajektorienplanung für automatisiertes Fahren im fahrdynamischen Grenzbereich : Eine modellbasierte Rundenzeitoptimierung für seriennahe Fahrzeuge. In: AUTOREG 2017. VDI-Berichte 2292. Düsseldorf: VDI-Verlag, Juli 2017, S. 223-234. ISBN: 978-3-18-092292-8.

M. Hafner, T. Hug, C. Hämmerling und U. Pressel: E-Klasse Assistenzsysteme in neuer Dimension. In: Sonderprojekte ATZ/MTZ, 21(Suppl. 1), Mai 2016, S. 70-80. DoI: 10.1007/s41491-016-0523-1. T. Hansen, M. Schulz, M. Knoop und U. Konigorski: Trajectory Planning for Automated Lane Changes. In: ATZ worldwide, 118(7), Juli 2016, S. 60-65. DOI: $10.1007 / \mathrm{s} 38311-016-0079-4$.

T. Hansen, M. Schulz, M. Knoop und U. Konigorski: Trajektorienplanung für automatisierte Fahrstreifenwechsel. In: ATZ - Automobiltechnische Zeitschrift, 118(7), Juli 2016, S. 66-71. DOI: 10 . 1007 /s35148-0160071-8.

T. Hansen, M. Schulz, M. Knoop und U. Konigorski: Kombinierte Längsund Quertrajektorienplanung für automatisierte Fahrstreifenwechsel. In: AUTOREG 2017 VDI-Berichte 2292. Düsseldorf: VDI-Verlag, Juli 2017, S. 235-246. ISBN: 978-3-18-092292-8.

M. Katke: Entwicklung realistischer Verkehrsumgebungen zum Testen von Fahrerassistenzsystemen für automatisierte Fahrstreifenwechsel. Bachelorarbeit. Technische Universität Darmstadt, Institut für Automatisierungstechnik und Mechatronik, Okt. 2014.

J. C. Lagarias, J. A. Reeds, M. H. Wright und P. E. Wright: Convergence Properties of the Nelder-Mead Simplex Method in Low Dimensions. In: SIAM Journal of Optimization, 9(1), 1998, S. 112-147. DoI: 10 . 1137/ S1052623496303470.

J. A. Nelder und R. Mead: A Simplex Method for Function Minimization. In: The Computer Journal, 7(4), Jan. 1965, S. 308-313. Dor: 10.1093/ comjnl/7.4.308.

J. Nilsson, M. Brännström, E. Coelingh und J. Fredriksson: Longitudinal and lateral control for automated lane change maneuvers. In: 2015 American Control Conference (ACC). Chicago, Juli 2015, S. 1399-1404. DOI: 10.1109/ACC.2015.7170929. 
[Nilsson, 2016]

[Nilsson, 2017]

[Rathgeber, 2016a]

[Rathgeber, 2016b]

[SAE J3016]

[Schmidt, 2005]

[Shanno, 1970]

[Treiber, 2010]

[Treiber, 2013]

[vStryk, 2014]
J. Nilsson, J. Silvlin, M. Brannstrom, E. Coelingh und J. Fredriksson: If, When, and How to Perform Lane Change Maneuvers on Highways. In: IEEE Intelligent Transportation Systems Magazine, 8(4), Okt. 2016, S. 68-78. DoI: 10.1109/MITS. 2016.2565718.

J. Nilsson, M. Brännström, E. Coelingh und J. Fredriksson: Lane Change Maneuvers for Automated Vehicles. In: IEEE Transactions on Intelligent Transportation Systems, 18(5), Mai 2017, S. 1087-1096. Dor: 10.1109/ TITS.2016.2597966.

[Papageorgiou, 2015] M. Papageorgiou, M. Leibold und M. Buss: Optimierung : Statische, dynamische, stochastische Verfahren für die Anwendung. 4., korr. Aufl. Berlin, Heidelberg: Springer-Verlag, 2015. isBn: 978-3-662-46935-4. DOI: $10.1007 / 978-3-662-46936-1$.

C. Rathgeber: Trajektorienplanung und -folgeregelung für assistiertes bis hochautomatisiertes Fahren. Dissertation. Technische Universität Berlin, Sep. 2016. DoI: 10.14279/depositonce-5506.

C. Rathgeber, F. Winkler und S. Müller: Kollisionsfreie Längs- und Quertrajektorienplanung unter Berücksichtigung fahrzeugspezifischer Potenziale. In: at - Automatisierungstechnik, 64(1), Jan. 2016, S. 61-76. DoI: $10.1515 /$ auto-2015-0052.

J3016 Taxonomy and Definitions for Terms Related to Driving Automation Systems for On-Road Motor Vehicles. SAE International, Sep. 2016. DoI: 10.4271/J3016_201609.

M. Schmidt: minFunc: unconstrained differentiable multivariate optimization in Matlab. 2005. URL: https : //www . cs . ubc . ca/ schmidtm/ Software/minFunc.html (abgerufen am 27. 04. 2017).

D. F. Shanno: Conditioning of Quasi-Newton Methods for Function Minimization. In: Mathematics of computation, 24(111), Juli 1970, S. 647-656. DOI: 10 . 1090/S0025-5718-1970-0274029-X.

M. Treiber und A. Kesting: Verkehrsdynamik und -simulation: Daten, Modelle und Anwendungen der Verkehrsflussdynamik. Berlin, Heidelberg: Springer-Verlag, 2010. ISBN: 978-3-642-05227-9. Dor: 10 . 1007/978-3642-05228-6.

M. Treiber und A. Kesting: Traffic Flow Dynamics: Data, Models and Simulation. Berlin, Heidelberg: Springer-Verlag, 2013. ISBN: 978-3-64232459-8. Dол: 10 . 1007/978-3-642-32460-4.

O. von Stryk: Vorlesungsfolien zu: Optimierung statischer und dynamischer Systeme, SoSe 2014. 2014. URL: http: //www . sim. informatik . tu-darmstadt.de (abgerufen am 13.11.2017). 
[Wendel, 2011]

[Werling, 2011]

[Winner, 2015]

[Ziegler, 2014]

[Ziegler, 2015]
J. Wendel: Integrierte Navigationssysteme : Sensordatenfusion, GPS und inertiale Navigation. 2., überarb. Aufl. München: Oldenbourg Wissenschaftsverlag, 2011. ISBN: 978-3-486-70439-6.

M. Werling: Ein neues Konzept für die Trajektoriengenerierung und stabilisierung in zeitkritischen Verkehrsszenarien. Karlsruhe: KIT Scientific Publishing, 2011. ISBN: 978-3-86644-631-1. DOI: 10 . 5445 / KSP / 1000021738.

H. Winner, S. Hakuli, F. Lotz und C. Singer, Hrsg.: Handbuch Fahrerassistenzsysteme: Grundlagen, Komponenten und Systeme für aktive Sicherheit und Komfort. 3. Auflage. Wiesbaden: Springer Vieweg, 2015. ISBN: 978-3-658-05733-6. DOI: 10 .1007/978-3-658-05734-3.

J. Ziegler, P. Bender, T. Dang und C. Stiller: Trajectory planning for Bertha - A local, continuous method. In: 2014 IEEE Intelligent Vehicles Symposium Proceedings. Dearborn, Juni 2014, S. 450-457. Dor: 10. $1109 /$ IVS. 2014.6856581.

J. Ziegler: Optimale Bahn- und Trajektorienplanung für Automobile. Dissertation. Karlsruher Institut für Technologie, 2015. DOI: 10.5445/ IR/1000057846. 